Iris Henning

\title{
Die Reputation einer Zentralbank
}




\section{Iris Henning}

\section{Die Reputation einer Zentralbank}

Kann die Europäische Zentralbank die hohe Reputation der Deutschen Bundesbank übernehmen? Für die Analyse dieser Frage wird das Grundmodell von Barro und Gordon dahingehend erweitert, daß Reputation auch dann aufgebaut werden kann, wenn die Antiinflationspolitik unglaubwürdig erscheint. Hierbei treten nun allerdings reale Kosten auf, die von der erwarteten und von der gesellschaftlich präferierten Inflationsrate abhängen.Vor diesem Hintergrund wird der Erfolg der Bundesbank nicht nur auf deren Unabhängigkeit, sondern auch auf die Stabilitätskultur in Deutschland zurückgeführt. Die Europäische Zentralbank wird sich dagegen heterogenen Stabilitätsauffassungen gegenübersehen. Es wird gezeigt, daß externe Anreize eine Antiinflationspolitik sicherstellen können.

Iris Henning, geb. Kirschbaum, wurde 1967 in Celle geboren. Von 1987 bis 1992 studierte sie Wirtschaftswissenschaften an der Universität Hannover. Nach dem Diplom arbeitete sie als wissenschaftliche Mitarbeiterin am Institut für Theoretische Volkswirtschaftslehre der Universität der Bundeswehr Hamburg, wo sie 1997 promovierte. 
Die Reputation einer Zentralbank

Iris Henning - 978-3-631-75125-1

Downloaded from PubFactory at 01/11/2019 08:02:27AM

via free access 


\section{SCHRIFTEN ZUR WIRTSCHAFTSTHEORIE UND WIRTSCHAFTSPOLITIK}

Herausgegeben von

Rolf Hasse, Wolf Schäfer, Thomas Straubhaar und Klaus W. Zimmermann

Band 3

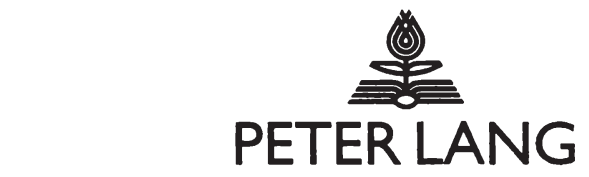

Frankfurt am Main - Berlin - Bern - New York - Paris - Wien 
Iris Henning

\title{
Die Reputation einer Zentralbank
}

\author{
Eine theoretische Untersuchung \\ unter besonderer Berücksichtigung \\ der Europäischen Zentralbank
}

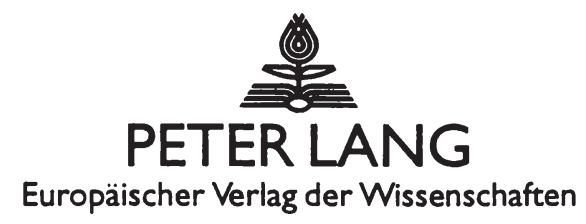

Iris Henning - 978-3-631-75125-1

Downloaded from PubFactory at 01/11/2019 08:02:27AM 
Die Deutsche Bibliothek - CIP-Einheitsaufnahme

Henning, Iris:

Die Reputation einer Zentralbank : eine theoretische Untersuchung unter besonderer Berücksichtigung der

Europäischen Zentralbank / Iris Henning. - Frankfurt am Main ; Berlin ; Bern ; New York ; Paris ; Wien : Lang, 1997

(Schriften zur Wirtschaftstheorie und Wirtschaftspolitik ; Bd. 3)

Zugl.: Hamburg, Univ. der Bundeswehr, Diss., 1997 ISBN 3-631-32123-6

Open Access: The online version of this publication is published on www.peterlang.com and www.econstor.eu under the international Creative Commons License CC-BY 4.0. Learn more on how you can use and share this work: http://creativecommons.org/licenses/ by/4.0.

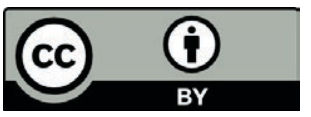

This book is available Open Access thanks to the kind support of ZBW - Leibniz-Informationszentrum Wirtschaft.

\author{
D 705 \\ ISSN 1433-1519 \\ ISBN 3-631-32123-6 \\ ISBN 978-3-631-75125-1 (eBook) \\ (C) Peter Lang GmbH \\ Europäischer Verlag der Wissenschaften \\ Frankfurt am Main 1997 \\ Alle Rechte vorbehalten.
}

Das Werk einschließlich aller seiner Teile ist urheberrechtlich geschützt. Jede Verwertung außerhalb der engen Grenzen des

Urheberrechtsgesetzes ist ohne Zustimmung des Verlages unzulässig und strafbar. Das gilt insbesondere für Vervielfältigungen, Übersetzungen, Mikroverfilmungen und die Einspeicherung und Verarbeitung in elektronischen Systemen.

Printed in Germany 123457 
Für Andreas und Alexander

Iris Henning - 978-3-631-75125-1

Downloaded from PubFactory at 01/11/2019 08:02:27AM

via free access 
Iris Henning - 978-3-631-75125-1

Downloaded from PubFactory at 01/11/2019 08:02:27AM

via free access 


\section{Vorwort}

Mit der Fertigstellung dieses Buches geht ein wichtiger Abschnitt meines Lebens zu Ende, der nicht nur für mich nicht immer einfach war. Deshalb möchte ich mich an dieser Stelle bei all denen bedanken, die mich während dieser Zeit begleitet und unterstützt haben.

Diese Arbeit entstand während meiner Tätigkeit am Institut für Theoretische Volkswirtschaftslehre der Universität der Bundeswehr Hamburg. An erster Stelle bedanke ich mich daher sehr herzlich bei meinem akademischen Lehrer, Herrn Prof. Dr. Wolf Schäfer, der es mir ermöglichte, Promotion und Familie miteinander in Einklang zu bringen. Des weiteren bedanke ich mich bei Herrn Prof. Dr. Rolf Hasse für die Übernahme des Zweitgutachtens. Schließlich bedanke ich mich bei den Herausgebern, Prof. Dr. Rolf Hasse, Prof. Dr. Wolf Schäfer, Prof. Dr. Thomas Straubhaar sowie Prof. Dr. Klaus Zimmermann, für die Aufnahme meiner Arbeit in diese Schriftenreihe.

Mein größter Dank aber gilt meinem lieben Mann und Kollegen Andreas, der mich immer wieder anspornte, meinen Weg fortzusetzen und nicht aufzugeben. Ich danke ihm für die vielen langen Diskussionen, daß er mir stets mit Rat und Tat zur Seite stand und so einen wesentlichen Beitrag zu dieser Arbeit leistete. Bedanken möchte ich mich aber auch bei meinem kleinen Sohn Alexander, der während dieser Zeit geboren wurde und der mich so gut hat arbeiten lassen, daß ich meine Promotion nicht aufgeben mußte. Als Alexander während der Endphase zunehmend neugieriger und mobiler wurde, haben insbesondere meine Mutter Edeltraut Baltruschat, aber auch mein Vater Hartmut Baltruschat sowie meine Schwester Martina Kirschbaum es mir durch mehrfache Übernahme seiner Betreuung ermöglicht, meine Arbeit zügig abzuschließen und mich in Ruhe auf das Rigorosum vorzubereiten. Hierfür möchte ich mich ganz herzlich bei ihnen bedanken.

Gedruckt mit Unterstützung der Universität der Bundeswehr Hamburg.

Hamburg, im Mai 1997

Iris Henning 
Iris Henning - 978-3-631-75125-1

Downloaded from PubFactory at 01/11/2019 08:02:27AM

via free access 


\section{Inhaltsverzeichnis}

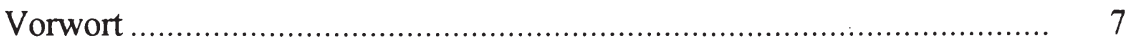

Verzeichnis der Abbildungen ............................................................... 11

Verzeichnis der Tabellen ................................................................... 13

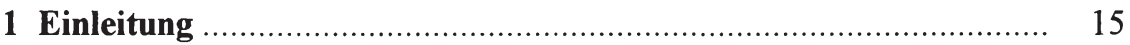

2 Die Bedeutung der Reputation ................................................. 17

2.1 Das Reputationsmodell von Barro/Gordon ............................... 17

2.2 Reputation, Prestige und Glaubwürdigkeit................................. 28

2.3 Das modifizierte Barro/Gordon-Modell ....................................... 36

3 Der Aufbau von Reputation ........................................................ 59

3.1 Die Leistung einer Zentralbank ................................................ 60

3.1.1 Erfahrungen als Indiz für die Leistung ............................ 60

3.1.2 Inflationsanreize .............................................................. 61

3.1.3. Die Bedeutung der Leistung für das Modell ......................... 64

3.2 Die Glaubwürdigkeit der Geldpolitik ....................................... 67

3.2.1 Das ,politische“ Glaubwürdigkeitsproblem ........................ 67

3.2.2 „Strategische“ Glaubwürdigkeitsprobleme.......................... 71

3.2.3 „Technologisches“ Glaubwürdigkeitsproblem .................... 79

3.2.4 Die Bedeutung der Glaubwürdigkeit für das Modell ............ $\quad 84$

3.2.5 Lösung der Glaubwürdigkeitsprobleme............................... 85

3.3 Die Unabhängigkeit einer Zentralbank ................................... 88

3.3.1 Unabhängigkeit als notwendige, aber nicht hinreichende Bedingung ................................................................. 88

3.3.2 Bestimmungsfaktoren der rechtlichen Unabhängigkeit ......... 89

3.3.3 Tatsächliche Unabhängigkeit ............................................ 93

3.3.4 Die Zentralbank als Eigennutzmaximierer.......................... 95

3.3.5 Die Bedeutung der Unabhängigkeit der Zentralbank für das

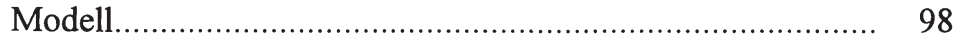

3.4 Anreize für Zentralbanker.................................................... 106

3.4.1 Kontrollmechanismen .................................................... 106

3.4.2 Externe Anreize .......................................................... 110

3.4.2.1 Abbau der Informationsasymmetrie ....................... 110

3.4.2.2 Machtbegrenzung durch Regelbindung .................... 112

3.4.2.3 Persönlich wirkende Anreize und Sanktionen............. 115

3.4.3 Die Bedeutung für das Modell ........................................ 122 
4 Die Reputation der Europäischen Zentralbank (EZB)................. 125

4.1 Die Leistung der potentiellen Mitgliedsländer............................. 125

4.2 Die Glaubwürdigkeit einer europäischen Geldpolitik .................. 134

4.3 Die Unabhängigkeit der EZB …............................................... 143

4.4 Anreize für die EZB ........................................................... 152

5 Zusammenfassung und abschließende Beurteilung ...................... 157

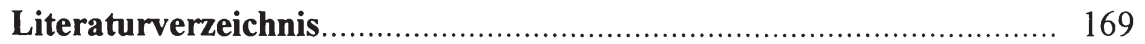




\section{Verzeichnis der Abbildungen}

Abbildung 2.1: Nutzen einer Überraschungsinflation............................ 21

Abbildung 2.2: Zwang und Anreiz..................................................... 27

Abbildung 2.3: Reputationsaufbau bei Glaubwürdigkeit (Barro/GordonModell) .................................................................... 35

Abbildung 2.4: Reputationsaufbau bei Glaubwürdigkeit und Unglaubwürdigkeit (modifiziertes Modell)................................ 36

Abbildung 2.5: Prestige und Reputation (modifiziertes Modell).............. 36

Abbildung 2.6: Abnehmender Anreiz zur Inflationierung bei einer

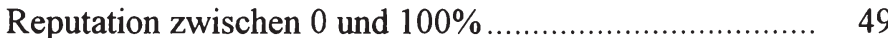

Abbildung 2.7: Zunehmender Anreiz zur Inflationierung bei einer

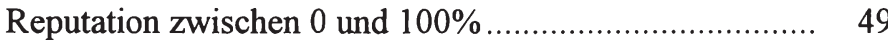

Abbildung 3.1: Inflationserwartungen, geplante und tatsächliche Inflation............................................................ 80

Abbildung 3.2: Mögliche Störungen der Geldpolitik ............................... $\quad 81$

Abbildung 3.3: Institutionelle Machtteilungsmechanismen..................... 86

Abbildung 3.4: Komponenten der rechtlichen Unabhängigkeit................ 90

Abbildung 4.1: Die Inflationsraten der EU-Länder von 1957 bis $1995 \ldots \ldots .128$ 
Iris Henning - 978-3-631-75125-1

Downloaded from PubFactory at 01/11/2019 08:02:27AM

via free access 


\section{Verzeichnis der Tabellen:}

Tabelle 3.1: Kosten der Politiker bei Sicherheit.................................. 73

Tabelle 3.2: $\quad$ Kosten der Wirtschaftssubjekte bei Sicherheit ................ 73

Tabelle 3.3: $\quad$ Kosten der Politiker bei Unsicherheit............................... 74

Tabelle 3.4: $\quad$ Prestige, Stabilitätsbewußtsein und Inflation ................. 101

Tabelle 3.5: Macht, Stabilitätsbewußtsein und Inflation ................... 103

Tabelle 4.1: Durchschnittliche Inflationsraten der EU-Mitglieder ....... 127

Tabelle 4.2: „Glaubwürdigkeit" der nationalen Geldpolitiken............. 136

Tabelle 4.3: "Glaubwürdige“ Antiinflationspolitik der EZB .............. 139

Tabelle 4.4: Rangfolge des Beitritts der EU-Länder zur Währungsunion entsprechend ihrer nationalen „Glaubwürdigkeit"... 141 
Iris Henning - 978-3-631-75125-1

Downloaded from PubFactory at 01/11/2019 08:02:27AM

via free access 
Mit der Unterzeichnung des Maastricht-Vertrags am 7. Februar 1992 durch die Staats- und Regierungschefs der Länder der Europäischen Union (EU) wurde die Gründung einer Europäischen Wirtschafts- und Währungsunion bis spätestens 1999 beschlossen. Der Wunsch nach einer Währungsunion ist keineswegs neu. Bereits 1969 sah der Werner-Plan die Gründung einer Währungsunion in drei Phasen bis 1980 vor. Genau wie dieser Versuch blieb aber auch der folgende, der 1979 mit Gründung des Europäischen Währungssystems (EWS) begann, bereits in der ersten Phase stecken. ${ }^{1}$ Mangelnde ökonomische Konvergenz sowie die fehlende Bereitschaft der Mitgliedstaaten zur Übertragung nationaler Hoheitsrechte waren die Ursache dafür, daß die Anläufe zur Währungsunion nicht über ihre Experimentierstadien hinausgingen.

Mit der Einheitlichen Europäischen Akte (EEA) von 1986, die die Vollendung des einheitlichen Binnenmarktes vorsah, kam erneut die Diskussion über die Notwendigkeit einer Europäischen Währungsunion auf. ${ }^{2}$ Die erste Phase des wiederum dreistufigen Prozesses auf dem Weg zur Währungsunion - mit einer Europäischen Zentralbank (EZB) - ist bereits abgeschlossen. Seit dem 1. Januar 1994 befinden die europäischen Länder sich in der direkten „Vorbereitungsphase“ zur Währungsunion.

Das Ziel dieser Arbeit ist es, ausgehend von der Annahme, daß die EZB tatsächlich gegründet wird, eine Analyse in bezug auf die Bestimmungsgrößen der potentiellen Reputation dieser Institution vorzunehmen. Hierfür wird zunächst die Frage geklärt, warum die Reputation einer Zentralbank überhaupt wichtig ist und wie sie entstehen kann. Die Reputation wird hierbei interpretiert als Ruf der Zentralbank in der Öffentlichkeit, ein Inflationsgegner zu sein. ${ }^{3}$ Eine hohe Reputation impliziert somit geringe Inflationserwartungen der Wirtschaftssubjekte. Eine Antiinflationspolitik ist dann glaubwürdig und kann durchgeführt werden, ohne daß zunächst hohe Erwartungen unter Inkaufnahme volkswirtschaftlicher Kosten ,gebrochen“ werden müssen. ${ }^{4}$

1 Zur Geschichte der europäischen Integration, insbesondere des Scheiterns der Versuche zur Gründung einer Währungsunion vgl. Hasse (1986), (1989b) und (1990).

2 Vgl. hierzu das Genscher-Memorandum vom 26.2.1988.

3 Diese Interpretation der Reputation einer Zentralbank ist in der geldtheoretischen Literatur üblich und wird hier entsprechend verwendet. Vgl. Barro/Gordon (1983a und b), Backus/Driffill (1985a und b) sowie Cukierman/Meltzer (1986a und b). Auf die Bedeutung der Glaubwürdigkeit für die Effizienz der Geldpolitik wies bereits Fellner (1976) und (1979) hin. 
Der Aufbau dieser Arbeit gliedert sich wie folgt: Im anschließenden zweiten Kapitel wird das von Barro/Gordon 1983 entwickelte Grundmodell zur Reputation vorgestellt, welches die große Bedeutung der Reputation nachweist. Anhand des Modells werden dann die Begriffe Reputation und Glaubwürdigkeit ausführlich definiert und voneinander abgegrenzt. Ein Problem ergibt sich jedoch durch die Einführung des Begriffs Prestige. Barro/Gordon setzen implizit die Begriffe Reputation - im Sinne von Inflationsaversion - und Prestige - im Sinne von Ansehen bzw. Beliebtheit bei der Bevölkerung - gleich. Sie betrachten also nur den Spezialfall, daß die Bevölkerung inflationsavers ist. Diese Annahme wird hier aufgegeben und eine Erweiterung des Modells insofern vorgenommen, als nun eine inhaltliche Differenzierung der beiden Begriffe Reputation und Prestige eingeführt wird. Zudem führt bei Barro/Gordon ausschließlich eine glaubwürdige Geldpolitik zum Aufbau von Reputation. In der Modellerweiterung wird auch die Möglichkeit des Reputationsaufbaus durch eine unglaubwürdige Geldpolitik einbezogen.

Im dritten Kapitel wird diskutiert, wie die die Reputation bestimmenden GröBen (die im zweiten Kapitel herausgearbeitet wurden) wiederum beeinflußt werden können bzw. wovon sie abhängen. Dies geschieht auf der Basis der Erkenntnisse der Neuen Politischen Ökonomie. Untersucht werden die Bedeutung der Leistung der Zentralbank, der Glaubwürdigkeit der Geldpolitik, der Unabhängigkeit der Zentralbank sowie externer Anreize für die Zentralbanker hinsichtlich des Aufbaus von Reputation.

Im vierten Kapitel werden die Ergebnisse des vorangegangenen Kapitels auf die EZB angewandt, d.h. die wesentlichen Determinanten des MaastrichtVertrags in bezug auf die EZB werden im Hinblick auf die Determinanten der Reputation analysiert. Da der Ausgangspunkt der Vergleich mit der Deutschen Bundesbank war, wird auch auf die Reputation der Bundesbank eingegangen. Im abschließenden fünften Kapitel wird nach einer kurzen Zusammenfassung aufgrund der vorangegangenen Überlegungen der Versuch einer Einschätzung der zu erwartenden Reputation der zukünftigen EZB unternommen. 
Die Grundlage für Reputationsüberlegungen in der Geldpolitik bildet das Zeitinkonsistenzproblem der optimalen Politik. Als zeitinkonsistent wird eine Politik bezeichnet, die $\mathrm{zu}$ einem Zeitpunkt $\mathrm{t}_{0}$ optimal erscheint, deshalb beabsichtigt und eventuell auch angekündigt wird, zum Zeitpunkt der Realisierung $t_{1}$ aber aufgrund neuer Rahmenbedingungen nicht mehr optimal ist.

Im Rahmen der Spieltheorie kann gezeigt werden, daß die ex ante optimale Politik nicht zeitkonsistent ist und deshalb auch nicht realisiert wird. Umgesetzt wird eine suboptimale Politik, die für die Gesellschaft zu Wohlfahrtseinbußen führt, andererseits aber die einzig zeitkonsistente und somit glaubwürdige Politik darstellt. Der Grund dieses „Dilemmas“ ist darin zu sehen, daß die Wirtschaftssubjekte bei ihrem gegenwärtigen Handeln nicht nur vergangene Erfahrungen berücksichtigen, sondern auch von ihren Erwartungen bezüglich der zukünftigen Politik beeinflußt werden, was die Annahme rationaler Erwartungen erfordert. 5

Anhand des folgenden einfachen Grundmodells kann dieses Problem veranschaulicht werden. Die Geldpolitik wird als Spiel zwischen zwei Spielern verstanden, der Zentralbank und den Wirtschaftssubjekten. Es liegt ein deterministischer Modellrahmen vor, in dem beide Spieler stets über die gleichen Informationen verfügen. Die Informationen sind also symmetrisch verteilt.

Die Wirtschaftssubjekte verhalten sich aufgrund identischer Präferenzen und gleicher Informationen bei rationalen Erwartungen homogen. Ihre Strategievariable sind die Inflationserwartungen $\left(\Pi^{\mathrm{e}}\right)$.

Die Zentralbank richtet die Geldpolitik an den gesellschaftlichen Präferenzen aus und kann über die Geldpolitik die Inflationsrate (П) vollständig kontrollieren. Aufgrund der Gemeinwohlorientierung sind die Präferenzen beider Spieler identisch.

Aus Vereinfachungsgründen wird der Zeithorizont auf eine Periode begrenzt. Die Zugfolge des Spiels ist genau festgelegt. Die Wirtschaftssubjekte bilden zu

5 Auf die Problematik der Zeitinkonsistenz in der Geldpolitik haben als erste Kydland/ Prescott (1977), S. 473-489 hingewiesen. Da die meisten nachfolgenden Modelle zur Zeitinkonsistenz in der Geldpolitik auf dem erweiterten Modell von Barro/Gordon basieren, wird dies hier formal vorgestellt. Einen Überblick über weitere Modelle zur Zeitinkonsistenz geben Blackburn/Christensen (1989) und Persson/Tabellini (1991). 
Beginn der Periode ihre Inflationserwartungen, aufgrund derer sie ihre Löhne aushandeln. Um keinen Reallohnverlust zu erleiden, geht die erwartete Preissteigerungsrate in die Nominallohnforderungen ein. Die Unternehmen können die Lohnsteigerungen voll auf die Preise überwälzen. Die ausgehandelten Lohnverträge gelten über die gesamte Periode. Erst nachdem die Wirtschaftssubjekte sich festgelegt haben, setzt die Zentralbank die tatsächliche Inflationsrate.

Die Wirtschaft wird durch eine um die Inflationserwartungen erweiterte Phillips-Kurve beschrieben, wie dies die folgende Gleichung zum Ausdruck bringt:

$$
\mathrm{U}_{\mathrm{t}}=\mathrm{U}_{\mathrm{t}}^{\mathrm{n}}-\alpha \cdot\left(\Pi_{\mathrm{t}}-\Pi_{\mathrm{t}}^{\mathrm{e}}\right) \quad \text { mit } \quad \alpha>0
$$

Die tatsächliche Arbeitslosenrate $\left(U_{t}\right)$ muß nicht der natürlichen Rate $\left(U_{t}^{n}\right)$ entsprechen, da erstere durch eine nicht antizipierte Inflation $\left(\Pi_{t}-\Pi_{t}^{e}\right)$ kurzfristig gesenkt werden kann. ${ }^{6}$ Die Zentralbank hat demnach die Möglichkeit, durch eine Überraschungsinflation den Output zu beeinflussen.

Eine wichtige Annahme des Modells ist, daß eine tatsächliche Arbeitslosenrate in Höhe der natürlichen Rate als zu hoch betrachtet wird. Der Grund dafür sind Verzerrungen am Arbeitsmarkt ${ }^{7}$, die dazu führen, daß für die Zentralbank ein Anreiz zur Erzeugung temporärer Beschäftigungseffekte besteht und es trotz Gemeinwohlorientierung zum Konflikt zwischen Zentralbank und Wirtschaftssubjekten kommt. Es ist nun die Aufgabe der Zentralbank, die für die Gesellschaft optimale Politik zu wählen, indem sie ihre Zielfunktion unter der $\mathrm{Ne}$ benbedingung der beschriebenen Phillips-Kurven-Relation optimiert. Die Ziel-

6 Diese konjunkturellen Schwankungen können über die Lucas-Angebotsfunktion oder auf Basis von Lohnrigiditäten erklärt werden. Vgl. Lucas (1972), (1973) sowie Fischer (1977).

7 Barro/Gordon erklären dies mit der Existenz von Steuern, Arbeitslosenunterstützung und ähnlichem mehr. Vgl. Barro/Gordon (1983a), S. 593 und (1983b), S. 105f. Die Verantwortung für diese Verzerrungen trägt also der (ausgeklammerte) Fiskalbereich. Vgl. Loef (1988), S. 365. Eine alternative Erklärung bietet Canzoneri, indem er argumentiert, daß große Gewerkschaften den Arbeitsmarkt dominieren. Ausführlicher dazu Canzoneri (1985), insbesondere S. 1058. Diese Verzerrungen führen zu Arbeitslosigkeit und somit Mindereinnahmen für die Regierung sowie andererseits zunehmenden Transferzahlungen. Diese Kosten stellen externe Effekte dar, die von den einzelnen Wirtschaftssubjekten, im Gegensatz zur Regierung, nicht berücksichtigt werden und deshalb zu Konflikten führen können. 
funktion wird als Kostenfunktion beschrieben und muß minimiert werden. Sie lautet:

$$
z_{t}=\frac{a}{2} \cdot \Pi_{t}^{2}-b \cdot\left(\Pi_{t}-\Pi_{t}^{e}\right) \quad a, b>0
$$

Der erste Term bringt die gesellschaftlichen Kosten einer Inflation zum Ausdruck, die insbesondere in den negativen Allokations- und Wachstumseffekten, aber auch den willkürlichen Umverteilungswirkungen einer Inflation zu sehen sind. Die quadratische Form impliziert, daß sowohl ein „Über-“ als auch „Unterschießen“ der als optimal betrachteten Inflationsrate negativ bewertet wird. Außerdem verursacht eine zunehmende Zielabweichung einen exponentiellen Anstieg des gesellschaftlichen Verlustes. Der Parameter a zeigt an, welches Gewicht den Kosten der Inflation beigemessen wird. Der zweite Term stellt den Inflationsnutzen dar, der von den Kosten abgezogen werden muß und aus Vereinfachungsgründen linear sei. ${ }^{8}$ Wie anhand von Gleichung (2.1) zu sehen ist, stiftet eine Überraschungsinflation $\left(\Pi>\Pi^{\mathrm{e}}\right)$ einen positiven Nutzen, indem sie kurzfristig eine tatsächliche Arbeitslosenrate ermöglicht, die unter der natürlichen Rate liegt. Der Nutzenparameter $b$, der aus Vereinfachungsgründen konstant gesetzt wird $(b=\bar{b})$, gibt hierbei die relative Gewichtung an, die der Wirtschaftsstimulierung beimessen wird. Je größer b ist, desto wichtiger ist der Zentralbank die Stimulierung der Wirtschaft.

Bei diskretionärer Politik, d.h. einem nicht-kooperativen Spiel zwischen Zentralbank und Wirtschaftssubjekten, sieht die Zentralbank die heutigen sowie alle zukünftigen Inflationserwartungen als gegeben an und wählt die Inflationsrate in Abhängigkeit ihrer Kosten. Sie wählt also die Rate, bei der ihre Kosten minimal werden. Mathematisch bedeutet dies, daß die erste Ableitung ihrer Kostenfunktion Null werden muß.9 Durch Umformung erhält man dann die optimale Inflationsrate bei diskretionärer Politik $\left(\hat{\Pi}_{\mathrm{t}}\right)$ :

$$
\mathrm{z}_{\mathrm{t}}=\frac{\mathrm{a}}{2} \cdot \hat{\Pi}_{\mathrm{t}}^{2}-\overline{\mathrm{b}} \cdot\left(\hat{\Pi}_{\mathrm{t}}-\hat{\Pi}_{\mathrm{t}}^{\mathrm{e}}\right)
$$

8 Ein Modell, in dem auch der Inflationsnutzen als quadratische Funktion dargestellt wird, hat Pearlman (1989) beschrieben. Er berücksichtigt außerdem explizit die Reputation des Politikers in der Zielfunktion, da dieser aufgrund seines Wunsches nach Wiederwahl der Reputation einen positiven Nutzen beimißt.

9 Die zweite Bedingung für ein Minimum ist erfüllt, weil die zweite Ableitung größer als Null ist. 


$$
\frac{\delta z_{t}}{\delta \hat{\Pi}_{t}}=a \cdot \hat{\Pi}_{t}-\bar{b}=0
$$

(2.3) $\quad \hat{\Pi}_{\mathrm{t}}=\frac{\overline{\mathrm{b}}}{\mathrm{a}}$

Inflationsrate bei diskretionärer Politik

Da die Wirtschaftssubjekte dieses Optimierungsproblem der Zentralbank kennen, wählen auch sie bei rationalen Erwartungen die diskretionäre Inflationsrate $\left(\Pi_{t}^{e}=\hat{\Pi}_{t}=\bar{b} / a\right)$, und die Überraschungsinflation im Gleichgewicht bleibt aus, so daß die Kosten bei diskretionärer Politik $\left(\hat{z}_{t}\right)$ nur noch von $\hat{\Pi}_{t}$ abhängig sind:

$$
\hat{\mathrm{z}}_{\mathrm{t}}=\frac{\mathrm{a}}{2} \cdot \frac{\overline{\mathrm{b}}^{2}}{\mathrm{a}^{2}}-\overline{\mathrm{b}} \cdot\left(\frac{\overline{\mathrm{b}}}{\mathrm{a}}-\frac{\overline{\mathrm{b}}}{\mathrm{a}}\right)
$$

(2.4) $\hat{\mathrm{z}}_{\mathrm{t}}=\frac{1}{2} \cdot \frac{\overline{\mathrm{b}}^{2}}{\mathrm{a}}$

diskretionäre Lösung

Dieses nicht-kooperative Gleichgewicht ist aber nicht Pareto-optimal, denn trotz positiver Inflationsrate kann die Arbeitslosigkeit nicht sinken, da die Inflation keine Überraschung für die Wirtschaftssubjekte war. Gelänge es der Zentralbank, sich im voraus an eine (optimale) Inflationsregel glaubhaft zu binden, könnte das Ergebnis verbessert werden. Ideal wäre die Bindung an eine Nullinflation:

(2.5) $\Pi_{t}^{*}=0$

Inflationsrate bei Regelbindung

Solange die Zentralbank jedoch noch einen diskretionären Spielraum besitzt, kann sie diese Rate nur ankündigen. Sie ist nicht daran gebunden. Wäre sie das, würden auch die Wirtschaftssubjekte ihre Erwartungen entsprechend setzen, d.h. $\Pi_{t}^{e}=\Pi_{t}^{*}=0$. Die Folge wären regelgebundene $\operatorname{Kosten}\left(z_{t}^{*}\right)$ von Null:

$$
\mathrm{z}_{\mathrm{t}}^{*}=\frac{\mathrm{a}}{2} \cdot 0-\overline{\mathrm{b}} \cdot(0-0)
$$

$$
\mathrm{z}_{\mathrm{t}}^{*}=0
$$

Idealfall

Sobald die Wirtschaftssubjekte ihre Inflationserwartungen aber entsprechend der Ankündigung der Zentralbank Null gesetzt hätten, bestände für diese der Anreiz, vertragsbrüchig zu werden. Das Ergebnis könnte durch eine positive 
Inflationsrate verbessert werden. Die ex ante optimale Politik $(\Pi=0)$ wäre aufgrund neuer Rahmenbedingungen $\left(\Pi^{\mathrm{e}}=0\right)$ ex post nicht mehr optimal, d.h. sie ist zeitinkonsistent. Als wohlfahrtsmaximierende Inflationsrate ergäbe sich wieder die diskretionäre Rate $\bar{b} / \mathrm{a}$, die bei Inflationserwartungen von Null zu folgenden Kosten führen würde:

$$
\begin{aligned}
\widetilde{z}_{\mathrm{t}} & =\frac{\mathrm{a}}{2} \cdot \frac{\overline{\mathrm{b}}^{2}}{\mathrm{a}^{2}}-\overline{\mathrm{b}} \cdot\left(\frac{\overline{\mathrm{b}}}{\mathrm{a}}-0\right) \\
\widetilde{\mathrm{z}}_{\mathrm{t}} & =\frac{1}{2} \cdot \frac{\bar{b}^{2}}{\mathrm{a}}-\frac{\overline{\mathrm{b}}^{2}}{\mathrm{a}} \\
\text { (2.7) } \quad \widetilde{\mathrm{z}}_{\mathrm{t}} & =-\frac{1}{2} \cdot \frac{\overline{\mathrm{b}}^{2}}{\mathrm{a}}
\end{aligned}
$$$$
\text { Betrugslösung }
$$

Die Kosten bei Betrug $\left(\widetilde{\mathrm{z}}_{\mathrm{t}}\right)$ wären demnach geringer als bei Regelbindung. Letztere stellt deshalb nur eine Second-best-Lösung dar. Den aus der Betrugslösung resultierenden Nutzen erhält man aus der Differenz der Kosten bei Realisierung der Regellösung und der Kosten bzw. Kostenersparnis bei Täuschung der Wirtschaftssubjekte:

$$
E\left[z_{t}^{*}-\widetilde{z}_{t}\right]=0-\left(-\frac{1}{2} \cdot \frac{\bar{b}^{2}}{a}\right)=\frac{1}{2} \cdot \frac{\bar{b}^{2}}{a}>0
$$

Sobald die Wirtschaftssubjekte ihre Erwartungen nach oben anpassen, nimmt der Nutzen aus der Überraschungsinflation ab, wie dies Abbildung (2.1) veranschaulicht. Je nach Höhe der Inflationserwartungen kann die Zentralbank eine Überraschungsinflation herbeiführen, die aufgrund positiver Beschäftigungseffekte zu Wohlfahrtsgewinnen führt. Der größte Nutzen, in Höhe von $\bar{b}^{2} / 2 \cdot a$, wird erzielt, wenn die Wirtschaftssubjekte eine Nullinflation erwarten und die Zentralbank die diskretionäre Rate setzt. Je höher die Wirtschaftssubjekte ihre Inflationserwartungen aber setzten, desto geringer wird der Nutzen aus der Inflation, da der Überraschungseffekt geringer wird und demzufolge die realen Effekte. Sobald die Wirtschaftssubjekte die diskretionäre Rate korrekt erwarten, stiftet die Inflation keinen Nutzen mehr, weil sie nicht mehr überraschend kommt. 
Abbildung 2.1: Nutzen einer Überraschungsinflation

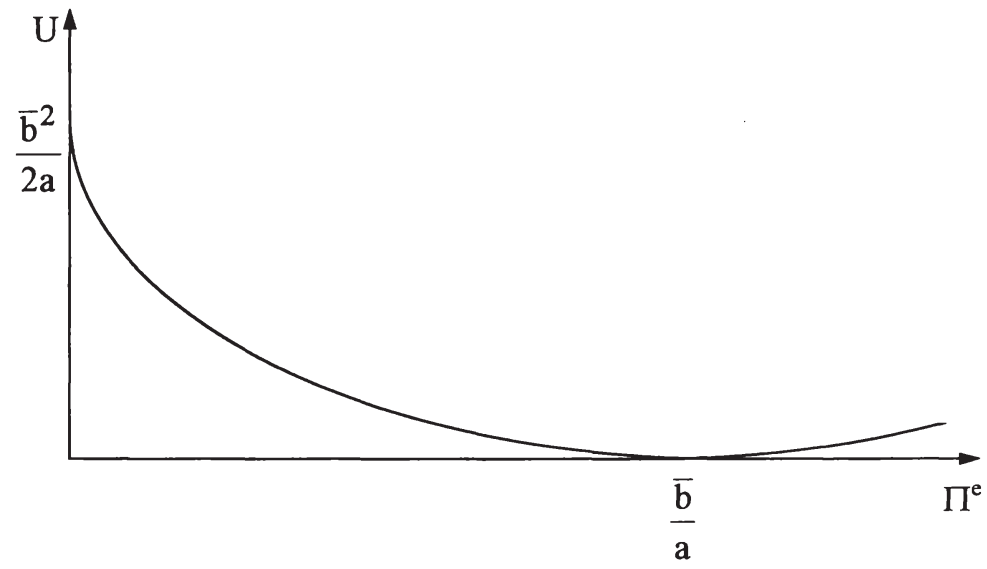

Da die Wirtschaftssubjekte dieses Anreizproblem kennen, werden sie ihre Erwartungen nicht Null setzen, solange die Zentralbank einen diskretionären Spielraum besitzt und an ihre Ankündigungen nicht gebunden ist, sondern gleich die diskretionäre Rate erwarten. Somit ist weder die Betrugslösung noch die Lösung bei Regelbindung erreichbar, sondern nur eine drittbeste mit gleicher Arbeitslosenrate, aber höherer Inflation als bei Regelbindung. ${ }^{10}$ Die Spieler befinden sich hier in einem Gefangenendilemma, da das Ergebnis zu verbessern wäre, wenn die Zentralbank sich nur glaubhaft binden könnte. Genau dies kann sie aber aufgrund des beschriebenen Anreizes nicht, da die Politik der Regelbindung zeitinkonsistent ist. Konsistent ist die suboptimale diskretionäre Politik, weil sie keinen Anreiz bietet, in der Zukunft von der gegenwärtigen Politik abzuweichen. Nur diese suboptimale Lösung stellt also ein Nash-Gleichgewicht dar. Es ist das einzige aufrechtzuerhaltende Gleichgewicht bei diskretionärer Politik. Bei rationalen Erwartungen können die Wirtschaftssubjekte nicht systematisch getäuscht werden, und deshalb müssen im Gleichgewicht die Erwartungen erfült werden $\left(\Pi=\Pi^{\mathrm{e}}\right)$. Nur hier entspricht der Grenznutzen der Überraschungsinflation auch den Grenzkosten der Inflation. Solange die Geldpolitiker über einen diskretionären Spielraum verfügen, können sie trotz Verfolgung einer sozialen Wohlfahrtsfunktion nur suboptimale Inflationsraten erzeugen, ohne dadurch aber die Arbeitslosigkeit zu mindern. Nur die Implementierung einer für die Geldpolitik verbindlichen Regel könnte

10 Vgl. Persson (1988), S. 520. Eine graphische Interpretation dieses Problems findet man bei Loef (1988), S. 368-372 und Jarchow (1993), S. 147-149. 
dieses „schlechte“ Ergebnis verhindern. ${ }^{11}$ Eine regelgebundene Politik kann die Erwartungen der Wirtschaftssubjekte stabilisieren, und eine geringere Inflationsrate kann realisiert werden. Die Arbeitslosigkeit verharnt allerdings auch hier auf ihrem natürlichen Niveau.

Barro/Gordon (BG) 12 haben aber gezeigt, daß die Einführung einer Regelbindung nicht unbedingt erforderlich ist, sobald Reputationsüberlegungen berücksichtigt werden. Wenn die Geldpolitik als fortlaufender Prozeß verstanden wird, in dem die geldpolitischen Entscheidungen und die Erwartungsbildung der Wirtschaftssubjekte in einem kontinuierlichen Wechselspiel zueinander stehen, ist ein intertemporaler Ansatz nötig. Das Einperiodenspiel, welches keine periodenübergreifenden Überlegungen zugelassen hat, muß durch ein Mehrperiodenspiel ersetzt werden. Zur Vereinfachung werden zwei Perioden betrachtet, die sich unendlich oft wiederholen können. ${ }^{13}$

Wie im einperiodigen Grundmodell bilden die Wirtschaftssubjekte ihre Erwartungen rational, und die Zentralbank versucht die gesellschaftliche Wohlfahrt $\mathrm{zu}$ maximieren. Sie hat dabei einen diskretionären Handlungsspielraum und kündigt ihre beabsichtigte Inflationsrate vorher an.

Im Gegensatz zum Einperiodenmodell muß nun eine gesellschaftliche Kostenfunktion minimiert werden, die mehrere Perioden berücksichtigt:

$$
\mathrm{Z}=\mathrm{E}\left[\mathrm{z}_{\mathrm{t}}+\frac{1}{1+\mathrm{r}} \cdot \mathrm{z}_{\mathrm{t}+1}\right]=\mathrm{E}\left[\mathrm{z}_{\mathrm{t}}+\mathrm{q} \cdot \mathrm{z}_{\mathrm{t}+1}\right]
$$

Die mehrperiodige Kostenfunktion $Z$ berücksichtigt nicht mehr nur die gegenwärtigen Kosten $\left(z_{t}\right)$, sondern auch die zukünftigen Kosten $\left(z_{t+1}\right)$, die noch mit der Diskontierungsrate $r$ bzw. dem Diskontierungsfaktor $q=1 / 1+r$ auf die heutige Periode abzudiskontieren sind. Der Faktor $r$ kann als Zeitpräferenzrate interpretiert werden. Demnach drückt $\mathrm{q}$, welches sich genau wie $\mathrm{r}$ zwischen Null und Eins bewegt, aus, wie wichtig der Zentralbank die Zukunft erscheint.

Für $\mathrm{z}_{\mathrm{t}}$ gilt wieder die bekannte Funktion:

$$
z_{t}=\frac{a}{2} \cdot \Pi_{t}^{2}-b \cdot\left(\Pi_{t}-\Pi_{t}^{e}\right)
$$

$$
\mathrm{a}, \mathrm{b}>0
$$

11 Vgl. Kydland/Prescott (1977), S. 487.

12 Diese Abkürzung ist in der Literatur üblich und wird deshalb auch hier verwendet.

13 Im Rahmen der Spieltheorie wird dies als Superspiel des Friedman-Typs bezeichnet. Vgl. Friedman (1971). 
Auch hier stiftet eine Überraschungsinflation einen positiven Nutzen, weil sie kurzfristig eine Beschäftigungsrate zuläßt, die über der natürlichen Rate liegt, welche wieder als zu hoch betrachtet wird. Entsprechend gilt die kurzfristige Phillips-Kurve:

$$
\mathrm{U}_{\mathrm{t}}=\mathrm{U}_{\mathrm{t}}^{\mathrm{n}}-\alpha \cdot\left(\Pi_{\mathrm{t}}-\Pi_{\mathrm{t}}^{\mathrm{e}}\right)
$$

mit $\alpha>0$

Im Gegensatz zum einperiodigen Grundmodell wird berücksichtigt, daß die bewußte Täuschung der Wirtschaftssubjekte nicht nur einen Nutzen in Form einer Überraschungsinflation, aus der reale Effekte resultieren, stiftet, sondern auch Kosten verursacht. Kündigt die Zentralbank die Nullinflation als ihre beabsichtigte Inflationsrate an, und die Wirtschaftssubjekte glauben ihr dies, indem sie ihre Erwartungen entsprechend setzen $\left(\Pi^{\mathrm{e}}=\Pi^{\mathrm{a}}=0\right)$, die aber von der Zentralbank enttäuscht werden, reagieren sie mit der Anpassung ihrer Erwartungen für die nächste Periode nach oben. Eine Überraschungsinflation verursacht nun nicht mehr nur einen Nutzen in Form der kurzfristigen Beschäftigungsstimulierung, sondern auch Kosten in Form steigender Inflationserwartungen in der Zukunft, was als Verlust der Reputation interpretiert wird. Die Zentralbank sieht sich bei ihrer Entscheidung über die optimale Inflationsrate einem Trade-off insofern gegenüber, als sie die heutigen Beschäftigungserfolge mit einem Reputationsverlust in der Zukunft bezahlen muß. Sobald also die Zukunft bei den geldpolitischen Entscheidungen berücksichtigt wird, nimmt die Inflationsneigung der Zentralbank $a b$, da eine Überraschungsinflation auch Kosten verursacht. Der Sanktionsmechanismus der Wirtschaftssubjekte wird dabei durch die folgende Erwartungsbildung ausgedrückt:

$$
\Pi_{t}^{e}=\left\{\begin{array}{lll}
\Pi_{t}^{*} & & \Pi_{t-1}=\Pi_{t-1}^{e} \\
\hat{\Pi}_{t} & \text { wenn } & \Pi_{t-1} \neq \Pi_{t-1}^{e}
\end{array}\right.
$$

Hat die Zentralbank die Erwartungen in der Vorperiode nicht enttäuscht, weil sie ihre Ankündigung eingehalten hat, erwarten die Wirtschaftssubjekte in der gegenwärtigen Periode wieder die Einhaltung der Regelbindung, d.h. die Zentralbank hat ihre Reputation behauptet. Sie verliert hingegen ihre Reputation, wenn sie die Erwartungen enttäuscht hat. Die Wirtschaftssubjekte glauben dann nicht mehr an die Einhaltung der Regelbindung, sondern erwarten die (schlechte) diskretionäre Lösung $\hat{\Pi}$. Will die Zentralbank dann keine Stabilisierungskrise herbeiführen, muß sie die hohen Inflationserwartungen mit einer entsprechend expansiven Geldpolitik akkommodieren. Die Folge ist eine ge- 
stiegene Inflationsrate bei konstanter Arbeitslosenrate. Andererseits gewinnt sie durch die Erfüllung der Erwartungen ihre Reputation zurück. Die Geldwertstabilität ist demnach für die Reputation hier nicht wichtig. In der Folgeperiode $\mathrm{t}+2$ kann wieder das optimale Ergebnis erzielt werden. Das Strafintervall für die Verletzung der Erwartungen beträgt genau eine Periode. Weder das Strafmaß noch die Straflänge hängen also von der Schwere des Vergehens ab.

Zur Bestimmung der Kosten des Vergehens muß die Differenz der eingetretenen Kosten bei diskretionärer Lösung $\left(\hat{z}_{t+1}\right)$ und der möglichen Kosten ohne Vergehen in $t$, d.h. der Kosten der Regelbindung $\left(z_{t+1}^{*}\right)$ betrachtet werden, die beide auf $\mathrm{t}$ abzudiskontieren sind: 14

(2.11) $E\left[\bar{q}_{t} \cdot\left(\hat{z}_{t+1}-z_{t+1}^{*}\right)\right]=\bar{q} \cdot\left(\frac{1}{2} \cdot \frac{\bar{b}^{2}}{a}-0\right)=\bar{q} \cdot \frac{1}{2} \cdot \frac{\bar{b}^{2}}{a}$

Kosten

Diese Differenz, die den erwarteten heutigen Wert des zukünftigen Verlustes ausdrückt, kann als Zwang zur Regeleinhaltung interpretiert werden. Im Gleichgewicht muß gelten: ${ }^{15}$

Anreiz zum Betrug = Zwang zur Regeleinhaltung

(2.12) $\frac{1}{2} \cdot \frac{\bar{b}^{2}}{\mathrm{a}}=\overline{\mathrm{q}} \cdot \frac{1}{2} \cdot \frac{\overline{\mathrm{b}}^{2}}{\mathrm{a}}$

Diese Gleichung ist nur bei $\overline{\mathrm{q}}=1$ erfüllt. Sobald aber $\overline{\mathrm{q}}<1$ gilt, ist der Anreiz zum Betrug größer als der Zwang zur Regeleinhaltung, und die Nullinflation ist keine Gleichgewichtslösung mehr, d.h. ihre Ankündigung ist nicht glaubwürdig.

Zur Ermittlung der Gleichgewichtslösung, also einer glaubwürdigen Inflationsankündigung, gilt als Ziel die Minimierung der erwarteten Kosten unter der Nebenbedingung der Gleichheit von Anreiz und Zwang sowie $\Pi_{\mathrm{t}}^{\text {opt }}=\Pi^{\mathrm{a}}=\Pi_{\mathrm{t}}^{*} \neq 0$ und $\Pi_{\mathrm{t}}^{\mathrm{e}}=\Pi^{\mathrm{a}}=\Pi_{\mathrm{t}}^{*}$ mit $\Pi^{\mathrm{a}}$ als angekündigter Inflationsrate. Es gilt:

$\Pi_{t}=\Pi_{t}^{*}$ und $\Pi_{t}^{e}=\Pi_{t}^{*}$

14 Aus Vereinfachungsgründen wird hier $q=\bar{q}=$ konstant gesetzt.

15 Der Anreiz zum Betrug wurde bereits im einperiodigen Grundmodell berechnet (Gleichung (2.8)) und kann hier übernommen werden. 
Kosten bei Regelbindung: $z_{t}^{*}=\frac{a}{2} \cdot \Pi_{t}^{* 2}-\bar{b} \cdot\left(\Pi_{t}^{*}-\Pi_{t}^{*}\right)=\frac{a}{2} \cdot\left(\Pi_{t}^{*}\right)^{2}$

$\Pi_{\mathrm{t}}=\frac{\overline{\mathrm{b}}}{\mathrm{a}}$ und $\Pi_{\mathrm{t}}^{\mathrm{e}}=\Pi_{\mathrm{t}}^{*}$

Kosten bei Betrug: $\widetilde{\mathrm{z}}_{\mathrm{t}}=\frac{\mathrm{a}}{2} \cdot \frac{\overline{\mathrm{b}}^{2}}{\mathrm{a}^{2}}-\overline{\mathrm{b}} \cdot\left(\frac{\overline{\mathrm{b}}}{\mathrm{a}}-\Pi_{\mathrm{t}}^{*}\right)=-\frac{1}{2} \cdot \frac{\overline{\mathrm{b}}^{2}}{\mathrm{a}}+\overline{\mathrm{b}} \cdot \Pi_{\mathrm{t}}^{*}$ $\Pi_{t}=\frac{\bar{b}}{a}$ und $\Pi_{t}^{e}=\frac{\bar{b}}{a}$

Kosten bei diskretionärer Politik: $\hat{z}_{t}=\frac{a}{2} \cdot \frac{\bar{b}^{2}}{a^{2}}-\bar{b} \cdot\left(\frac{\bar{b}}{a}-\frac{\bar{b}}{a}\right)=\frac{1}{2} \cdot \frac{\bar{b}^{2}}{a}$

Zur Ermittlung der Anreizfunktion muß wieder die Differenz der Kosten bei Regelbindung, also bei Einhaltung der Ankündigung, und Betrug betrachtet werden:

(2.13) Anreiz: $\mathrm{E}\left[\mathrm{z}_{\mathrm{t}}^{*}-\widetilde{\mathrm{z}}_{\mathrm{t}}\right]=\frac{\mathrm{a}}{2} \cdot \Pi_{\mathrm{t}}^{* 2}-\left(\frac{\mathrm{a}}{2} \cdot \frac{\overline{\mathrm{b}}^{2}}{\mathrm{a}^{2}}-\frac{\overline{\mathrm{b}}^{2}}{\mathrm{a}}+\overline{\mathrm{b}} \cdot \Pi_{\mathrm{t}}^{*}\right)$

$$
=\frac{\mathrm{a}}{2} \cdot\left(\frac{\bar{b}}{\mathrm{a}}-\Pi_{\mathrm{t}}^{*}\right)^{2}
$$

Nutzen

Die Zwangfunktion zur Regeleinhaltung ergibt sich wieder als Differenz der Kosten bei diskretionärer Politik und bei Einhaltung der Regelbindung. Da diese Kosten erst in der Zukunft anfallen würden, sind sie wieder mit $\overline{\mathrm{q}}$ abzudiskontieren. Man erhält:

(2.14) Zwang: $\overline{\mathrm{q}} \cdot \mathrm{E}\left[\hat{\mathrm{z}}_{\mathrm{t}+1}-\mathrm{z}_{\mathrm{t}+1}^{*}\right]=\overline{\mathrm{q}} \cdot\left[\frac{1}{2} \cdot \frac{\overline{\mathrm{b}}^{2}}{\mathrm{a}}-\frac{\mathrm{a}}{2} \cdot \Pi_{\mathrm{t}}^{* 2}\right]$

$$
=\overline{\mathrm{q}} \cdot \frac{\mathrm{a}}{2} \cdot\left[\left(\frac{\overline{\mathrm{b}}}{\mathrm{a}}\right)^{2}-\Pi_{\mathrm{t}}^{* 2}\right]
$$

Kosten

Anhand der graphischen Darstellung beider Funktionen in Abbildung (2.2) wird ersichtlich, daß nun die Ankündigung einer geringeren Inflationsrate als der diskretionären glaubwürdig ist und somit ein Gleichgewicht darstellt. Es gibt ein ganzes Lösungsintervall für $\Pi$, welches zwischen der schlechtesten diskretionären Lösung ( $\bar{b} / \mathrm{a})$ und der idealen Nullösung liegt. Letztere selbst stellt keine Lösung dar, weil hier der Nutzen aus einer Überraschungsinflation 
Abbildung 2.2: Zwang und Anreiz

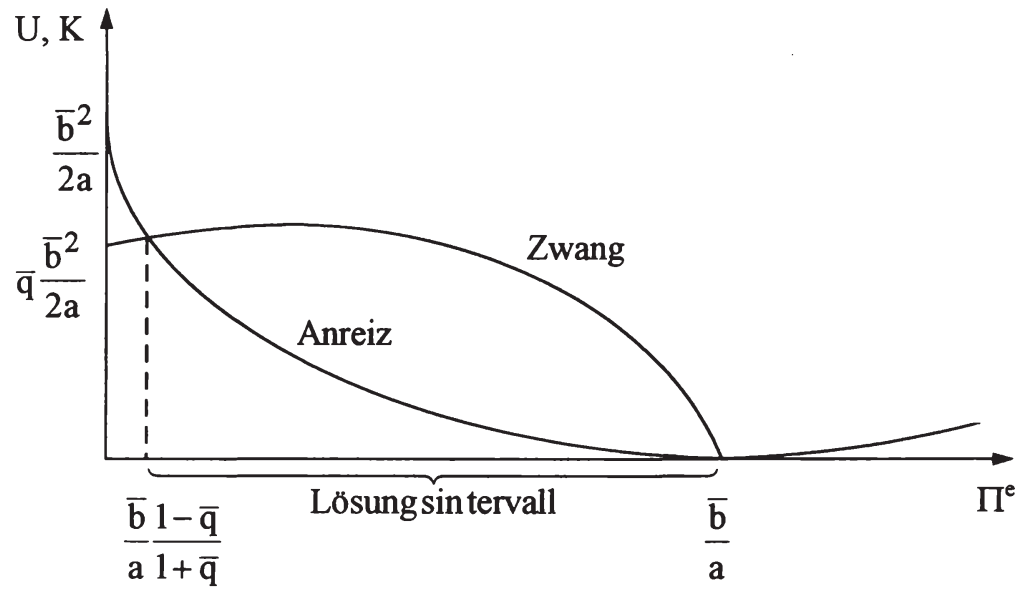

Quelle: Vgl. BG (1983a), S. 112.

größer ist als die daraus resultierenden zukünftigen Kosten. Da dies auch die "rationalen" Wirtschaftssubjekte wissen, werden sie ihre Erwartungen nicht Null setzen. Das Lösungsintervall ist dadurch gekennzeichnet, daß hier der Zwang zur Regeleinhaltung stets größer ist als der Anreiz zur Inflation. Die optimal erreichbare Inflationsrate ergibt sich als erster Schnittpunkt der beiden Funktionen. Man erhält sie durch Gleichsetzen der Anreiz- und Zwangfunktion:

$$
\frac{\mathrm{a}}{2} \cdot\left(\frac{\overline{\mathrm{b}}}{\mathrm{a}}-\Pi_{\mathrm{t}}^{*}\right)^{2}=\overline{\mathrm{q}} \cdot \frac{\mathrm{a}}{2} \cdot\left[\left(\frac{\overline{\mathrm{b}}}{\mathrm{a}}\right)^{2}-\Pi_{\mathrm{t}}^{* 2}\right]
$$

Die gesuchte Inflationsrate wird somit durch folgenden Ausdruck beschrieben:

(2.15) $\Pi_{t}^{*}=\frac{\bar{b}}{a} \cdot \frac{1-\bar{q}}{1+\bar{q}}$

Bei Wahl dieser Inflationsrate fallen für die Gesellschaft folgende Kosten an:

(2.16) $E\left[z_{t}^{*}\right]=\frac{a}{2} \cdot\left(\frac{\bar{b}}{a} \cdot \frac{1-\bar{q}}{1+\bar{q}}\right)^{2}-\bar{b} \cdot\left(\frac{\bar{b}}{a} \cdot \frac{1-\bar{q}}{1+\bar{q}}-\frac{\bar{b}}{a} \cdot \frac{1-\bar{q}}{1+\bar{q}}\right)=\frac{1}{2} \cdot \frac{\bar{b}^{2}}{a} \cdot\left(\frac{1-\bar{q}}{1+\bar{q}}\right)^{2}$ 
Falls $\bar{q}$ sich zwischen Null und Eins bewegt, liegt die Inflationsrate $\Pi_{t}^{*}$, die angekündigt und auch geglaubt wird, zwischen der idealen Regelbindung ( $\Pi$ $=0)$ und der diskretionären Lösung $(\Pi=\bar{b} / a)$. Sie ist ein gewichteter Durchschnitt aus beiden Werten mit $\bar{q}$ als Gewichtungsfaktor. Je höher $\bar{q}$ gewählt wird, d.h. je langfristiger die Geldpolitik orientiert ist, desto mehr kann der Reputationseffekt die gesellschaftliche Wohlfahrt steigern, da die realisierbare Inflationsrate mit steigendem $\bar{q}$ gegen Null tendiert. Gleiches gilt für eine stärkere Betonung von a relativ zu b, da dies das Stabilitätsbewußtsein zum Ausdruck bringt.

Der informelle Reputationsmechanismus verbessert also die pessimistische Lösung des Einperiodenmodells, da trotz des diskretionären Spielraums der Geldpolitiker eine bessere als die drittbeste Lösung $(\Pi=b / a)$ möglich wird, wenngleich auch hier die zweitbeste Lösung $(\Pi=0)$ nicht erreicht werden kann. ${ }^{16}$

BG haben mit ihrem Modell also gezeigt, daß eine Zentralbank mit Reputation eine Antiinflationspolitik durchführen kann, ohne daß sie dafür volkswirtschaftliche Kosten in Form einer temporär steigenden Arbeitslosigkeit in Kauf nehmen muß. Alle folgenden Reputationsmodelle haben zwar Erweiterungen und Verfeinerungen in vielfacher Hinsicht vorgenommen, diese grundsätzliche Aussage des BG-Modells blieb aber unverändert, was die noch heute große Bedeutung dieses Modells in der Reputationsliteratur erklärt.

\subsection{Reputation, Prestige und Glaubwürdigkeit}

Sowohl im BG-Modell als auch teilweise in seinen Erweiterungen werden die Begriffe Reputation und Glaubwürdigkeit nicht klar voneinander abgegrenzt, sondern oft synonym verwendet. Deshalb soll nun zunächst anhand des BGModells dargestellt werden, wie diese Begriffe hier verwendet werden.

Im BG-Modell führt ausschließlich eine glaubwürdige Geldpolitik zum Aufbau von Reputation (R). Dabei werden nur die beiden Extremfälle Existenz bzw. Nichtexistenz von Reputation unterstellt. Die Zentralbank realisiert entweder die geringstmögliche Inflationsrate $\left(\Pi^{\mathrm{opt}}\right)$ oder die schlechteste, also die höchste (b/a). Entsprechend erwarten die Wirtschaftssubjekte nur eine dieser beiden Alternativen $\left(\Pi^{\mathrm{e}}=\Pi^{\text {opt }}\right.$ oder $\left.\Pi^{\mathrm{e}}=\mathrm{b} / \mathrm{a}\right)$. Stimmen die Erwartungen der Wirtschaftssubjekte mit der Realisation nicht überein, kommt es zur Überra-

16 Vgl. BG (1983a), S.104-114. Eine andere Spezifikation der Verlustfunktion oder des Erwartungsmechanismus kann dazu führen, daß die Ankündigung der Nullinflation glaubwürdig ist. Vgl. BG (1983a), S. 110 und Rogoff (1987a). 
schungsinflation $\left(\Pi^{\mathrm{ZB}}>\Pi^{\mathrm{e}}\right)$ oder Überraschungsdeflation $\left(\Pi^{\mathrm{ZB}}<\Pi^{\mathrm{e}}\right)$. Der erste Fall kann kurzfristig zur Realisation einer Arbeitslosenrate führen, die unterhalb der natürlichen Rate liegt. Entsprechend führt eine Überraschungsdeflation zu einer Arbeitslosenrate, die die natürliche übersteigt. Angekündigt wird ausschließlich die optimale Inflationsrate $\left(\Pi^{\mathrm{a}}=\Pi^{\mathrm{opt}}\right)$. Anhand eines Zeitpfades lassen sich die Begriffe verdeutlichen:

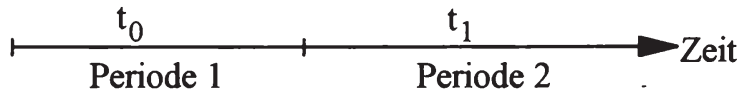

1. $\Pi^{\mathrm{a}}=\Pi^{\mathrm{opt}}=\Pi_{0}^{\mathrm{e}}=\Pi_{0}^{\mathrm{ZB}}$

$\rightarrow \mathrm{R}>0 \rightarrow \Pi^{\mathrm{a}}=\Pi^{\mathrm{opt}}=\Pi_{1}^{\mathrm{e}}=\Pi_{1}^{\mathrm{ZB}}$

2. $\Pi^{\mathrm{a}}=\Pi^{\mathrm{opt}}<\Pi_{0}^{\mathrm{e}}=\frac{\mathrm{b}}{\mathrm{a}}=\Pi_{0}^{\mathrm{ZB}}$

$\rightarrow \mathrm{R}>0 \rightarrow \Pi^{\mathrm{a}}=\Pi^{\mathrm{opt}}=\Pi_{1}^{\mathrm{e}}=\Pi_{1}^{\mathrm{ZB}}$

3. $\Pi^{\mathrm{a}}=\Pi^{\mathrm{opt}}=\Pi_{0}^{\mathrm{e}}<\Pi_{0}^{\mathrm{ZB}}=\frac{\mathrm{b}}{\mathrm{a}}$

$\rightarrow \mathrm{R}=0 \rightarrow \Pi^{\mathrm{a}}=\Pi^{\mathrm{opt}}<\Pi_{1}^{\mathrm{e}}=\frac{\mathrm{b}}{\mathrm{a}}=\Pi_{1}^{\mathrm{ZB}}$

4. $\Pi^{\mathrm{a}}=\Pi^{\mathrm{opt}}<\Pi_{\mathrm{o}}^{\mathrm{e}}=\frac{\mathrm{b}}{\mathrm{a}}>\Pi_{0}^{\mathrm{ZB}}=\Pi^{\mathrm{opt}} \rightarrow \mathrm{R}=0 \rightarrow \Pi^{\mathrm{a}}=\Pi^{\mathrm{opt}}<\Pi_{1}^{\mathrm{e}}=\frac{\mathrm{b}}{\mathrm{a}}=\Pi_{1}^{\mathrm{ZB}}$

Im ersten Fall wird die optimale Inflationsrate angekündigt, von den Wirtschaftssubjekten erwartet und von der Zentralbank realisiert. Die Übereinstimmung der Erwartungen der Wirtschaftssubjekte mit der tatsächlichen Realisation der Zentralbank wird hier als glaubwürdige Politik $\left(\Pi^{\mathrm{e}}=\Pi^{\mathrm{ZB}}\right)$ interpretiert. Ausschließlich die Glaubwürdigkeit der Geldpolitik führt zur Reputation. Diese äußert sich darin, daß die Wirtschaftssubjekte in der nächsten Periode die geringste Inflationsrate $\left(\Pi^{\mathrm{opt}}\right)$ erwarten, die Zentralbank also für einen Inflationsgegner halten. Die realisierte Inflationsrate ist in beiden Perioden die optimale, während die Beschäftigung auf ihrem natürlichen Niveau verharrt.

Im zweiten Fall glauben die Wirtschaftssubjekte nicht an die angekündigte optimale Inflationsrate, sondern erwarten die höhere Rate b/a. Wenn die Zentralbank eine glaubwürdige Politik durchführt und die Erwartungen erfüllt, indem sie die hohe Rate b/a realisiert, bringt ihr dies für die nächste Periode Reputation. Die Wirtschaftssubjekte erwarten dann für die nächste Periode die Durchführung der optimalen Inflationsrate, welche die Zentralbank realisiert und so ihre Reputation behält. Da die schlechte Inflationsrate in der ersten Periode erwartet wurde und somit keine Überraschung für die Wirtschaftssubjekte darstellt, kann sie nicht zur Minderung der Arbeitslosigkeit beitragen, d.h. realisiert werden die schlechteste Inflationsrate und die natürliche Arbeitslosenrate. 
Im dritten Fall besitzt die Zentralbank bereits in der ersten Periode Reputation, weil die Wirtschaftssubjekte die angekündigte optimale Rate erwarten. Tatsächlich realisiert die Zentralbank aber die höchste Rate b/a, um durch die Überraschungsinflation $\left(\Pi^{\mathrm{ZB}}>\Pi^{\mathrm{e}}\right)$ die Arbeitslosigkeit unter die natürliche Rate zu senken. Andererseits verliert sie durch diese unglaubwürdige Politik ihre Reputation, und die Wirtschaftssubjekte erwarten in der nächsten Periode die schlechteste Inflationsrate, welche die Zentralbank dann auch setzt. Die Realisation dieser hohen Inflationsrate führt nun zwar nicht mehr zur Minderung der Arbeitslosigkeit, da sie aber notwendig war für eine glaubwürdige Geldpolitik, führt sie wieder zur Reputation der Zentralbank.

Im letzten Fall erwarten die Wirtschaftssubjekte wegen der fehlenden Reputation der Zentralbank die Realisation der schlechtesten Inflationsrate b/a, die die Zentralbank aber nicht realisiert. Sie setzt die angekündigte optimale Inflationsrate. Da diese Geldpolitik allerdings nicht glaubwürdig ist, erlangt die Zentralbank so auch keine Reputation, muß aber trotzdem durch die Herbeiführung einer Überraschungsdeflation $\left(\Pi^{\mathrm{e}}>\Pi^{\mathrm{ZB}}\right)$ die Minderung der Beschäftigung unterhalb ihres natürlichen Niveaus verantworten. In der zweiten Periode erwarten die Wirtschaftssubjekte trotz der Realisation der optimalen Inflationsrate in der Vorperiode wieder die Durchführung der schlechtesten Rate b/a, die die Zentralbank diesmal auch durchführen wird, um so Reputation aufzubauen. Aufgrund der ausschließlich negativen Wirkungen in der ersten Periode kann der vierte Fall ausgeschlossen werden.

An diesem Modell ist zu kritisieren, daß ausschließlich eine - im Sinne der Erfüllung der Erwartungen der Wirtschaftssubjekte - glaubwürdige Geldpolitik zum Aufbau von Reputation führt. Dabei ist es unwesentlich, ob die beste oder schlechteste Inflationsrate erwartet wird. Eine Zentralbank gilt demnach auch dann als Inflationsgegner, wenn sie in der Vorperiode eine hohe Inflationsrate setzen mußte, um so eine glaubwürdige Politik zu realisieren.

$\mathrm{Da}$ der Aufbau von Reputation durch die Realisierung einer hohen Inflationsrate unrealistisch ist, wird der Begriff Glaubwürdigkeit in der nachfolgenden Modifizierung des Modells anders interpretiert. Bisher war eine Politik glaubwürdig, wenn die Zentralbank die von den Wirtschaftssubjekten erwartete Inflationsrate auch realisierte, unabhängig von der angekündigten Rate. Nun wird eine Politik als glaubwürdig definiert, wenn die Wirtschaftssubjekte glauben, daß die angekündigte Rate auch realisiert wird, unabhängig davon, ob dies dann tatsächlich geschieht. Bei BG handelt es sich somit um eine Ex postGlaubwürdigkeit, weil die Glaubwürdigkeit der Geldpolitik sich erst nach Realisation der Inflationsrate ergibt. In der Modifizierung handelt es sich dagegen 
um eine Ex ante-Glaubwürdigkeit, da die Qualifizierung vor Realisation der Inflationsrate erfolgt.

In der modifizierten Version von BG kann prinzipiell jede Inflationsrate zwischen den beiden Extremlösungen angekündigt werden. Da jedoch nur eine Antiinflationspolitik als glaubwürdige Geldpolitik interpretiert wird, muß die angekündigte Inflationsrate geringer sein als die in der Vorperiode realisierte Rate, solange die optimale Rate noch nicht erreicht ist. Die Zentralbank kann jede beliebige Inflationsrate zwischen $\Pi^{\text {opt }}$ und b/a setzen. Gleiches gilt für die Erwartungen der Wirtschaftssubjekte. Nur wenn die Erwartungen mit der Ankündigung übereinstimmen, liegt eine glaubwürdige Geldpolitik vor. Wird die angekündigte Rate von der Zentralbank dann realisiert, kann sie ihre Reputation steigern, die hier zwischen 0 und 100\% liegen kann und auch hier in der absoluten Höhe der Inflationserwartungen zum Ausdruck kommt. Wieder werden die unterschiedlichen Alternativen an einem Zeitstrahl verdeutlicht:

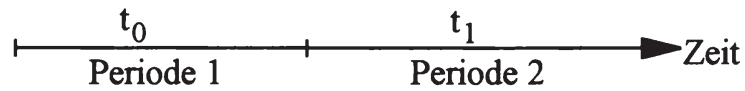

1. $\Pi_{-1}^{\mathrm{ZB}}>\Pi_{0}^{\mathrm{a}}=\Pi_{0}^{\mathrm{e}}=\Pi_{0}^{\mathrm{ZB}} \quad \rightarrow \mathrm{R} \uparrow \quad \rightarrow \quad \Pi_{0}^{\mathrm{a}}>\Pi_{1}^{\mathrm{a}}=\Pi_{1}^{\mathrm{e}}=\Pi_{1}^{\mathrm{ZB}}$

2. $\Pi_{-1}^{\mathrm{ZB}}>\Pi_{0}^{\mathrm{a}}<\Pi_{0}^{\mathrm{e}}=\Pi_{0}^{\mathrm{ZB}} \rightarrow \mathrm{R}=$ konst. $\rightarrow \Pi_{0}^{\mathrm{a}}>\Pi_{1}^{\mathrm{a}}<\Pi_{1}^{\mathrm{e}}>\Pi_{1}^{\mathrm{ZB}} \rightarrow \mathrm{R} \uparrow$

3. $\Pi_{-1}^{\mathrm{ZB}}>\Pi_{0}^{\mathrm{a}}<\Pi_{0}^{\mathrm{e}}>\Pi_{0}^{\mathrm{ZB}}>\Pi_{0}^{\mathrm{a}} \rightarrow \mathrm{R} \uparrow \quad \rightarrow \Pi_{1}^{\mathrm{a}}<\Pi_{1}^{\mathrm{e}}<\Pi_{\mathrm{o}}^{\mathrm{e}}$

aber: $\Pi_{1}^{\mathrm{e}}>\Pi_{0}^{\mathrm{a}}$ falls: $\Pi_{1}^{\mathrm{e}}>\Pi_{1}^{\mathrm{ZB}} \rightarrow \mathrm{R} \uparrow$

4. $\Pi_{-1}^{\mathrm{ZB}}>\Pi_{0}^{\mathrm{a}}<\Pi_{0}^{\mathrm{e}}>\Pi_{0}^{\mathrm{ZB}}=\Pi_{0}^{\mathrm{a}} \rightarrow \mathrm{R} \uparrow \quad \rightarrow \Pi_{1}^{\mathrm{a}}<\Pi_{1}^{\mathrm{e}}=\Pi_{0}^{\mathrm{a}}>\Pi_{1}^{\mathrm{ZB}} \rightarrow \mathrm{R} \uparrow$

5. $\Pi_{-1}^{\mathrm{ZB}}>\Pi_{0}^{\mathrm{a}}=\Pi_{0}^{\mathrm{e}}<\Pi_{0}^{\mathrm{ZB}} \rightarrow \mathrm{R} \downarrow \quad \rightarrow \Pi_{1}^{\mathrm{a}}<\Pi_{1}^{\mathrm{e}}=\Pi_{0}^{\mathrm{ZB}}>\Pi_{1}^{\mathrm{ZB}} \rightarrow \mathrm{R} \uparrow$

Im ersten Fall liegt eine glaubwürdige Politik vor, weil die Wirtschaftssubjekte an die Ankündigung der Zentralbank glauben. Erfüllt die Zentralbank diese Erwartung, die unterhalb der letzten Realisation liegt, steigt ihre Reputation. Kündigt die Zentralbank für die Folgeperiode eine noch geringere Inflationsrate an, halten die Wirtschaftssubjekte dies aufgrund ihrer positiven Erfahrungen für glaubwürdig. Realisiert die Zentralbank diese Rate wiederum, kann ihre Reputation weiter steigen. Die Inflationsraten können so von Periode zu Periode sinken, bis schließlich die optimale Rate realisiert wird. Die Arbeitslosenrate wird hiervon nicht beeinflußt, da die Wirtschaftssubjekte keine Überraschungen erleben. 
Im zweiten Fall glauben die Wirtschaftssubjekte der Ankündigung der Zentralbank in der ersten Periode nicht, folglich liegt eine unglaubwürdige Politik vor. Erfüllt die Zentralbank nun die Erwartungen der Wirtschaftssubjekte, kann sie so ihre Reputation nicht steigern, da sie ihre Ankündigung nicht eingehalten hat. Die noch geringere Ankündigung für die zweite Periode ist dann wieder nicht glaubwürdig für die Wirtschaftssubjekte. Es hängt nun von der Zentralbank ab, ob sie ihre Reputation steigern will, indem sie eine Inflationsrate unterhalb der erwarteten setzt, so aber auch eine Überraschungsdeflation mit Arbeitslosigkeit verursacht, oder ob sie die Erwartungen wieder erfüllt, um nicht von der natürlichen Arbeitslosenrate abzuweichen. Allerdings wird im zweiten Fall ihre Politik immer unglaubwürdiger, was letztlich zu einem Verlust von Reputation führen wird.

Auch im dritten Fall halten die Wirtschaftssubjekte die Ankündigung der Zentralbank für unglaubwürdig, da sie eine Inflationsrate erwarten, die oberhalb der angekündigten liegt. Realisiert die Zentralbank nun aber eine Rate, die unterhalb der Erwartungen liegt, kann sie so Reputation aufbauen. Allerdings realisiert die Zentralbank hier eine Rate, die oberhalb ihrer eigenen Ankündigung liegt, damit die Überraschungsdeflation und die daraus resultierende Arbeitslosigkeit nicht $\mathrm{zu}$ gravierend ausfallen. Kündigt die Zentralbank in der zweiten Periode eine Inflationsrate an, die noch unterhalb ihrer ersten Ankündigung liegt, werden die Wirtschaftssubjekte dies zwar wieder für unglaubwürdig halten, andererseits glauben sie an eine Realisation in Höhe der vergangenen Leistung $\left(\Pi_{0}^{Z B}\right)$, die unterhalb der Erwartungen in $t_{0}$ lag. Unterschreitet die Zentralbank nun wieder die Erwartungen, kann sie ihre Reputation weiter steigern. Dies verursacht zwar wieder Kosten in Form von Arbeitslosigkeit, andererseits erwarten die Wirtschaftssubjekte in der Folgeperiode dann die Realisation einer noch geringeren Inflationsrate.

Im vierten Fall glauben die Wirtschaftssubjekte wieder nicht an die angekündigte Rate der Zentralbank, werden von dieser aber damit überrascht, daß sie ihre Ankündigung tatsächlich realisiert. Unterstellt man eine identische Höhe der Inflationserwartungen im dritten und vierten Fall für die erste Periode, sind die Kosten in Form der Arbeitslosigkeit im vierten Fall höher, da die Differenz aus erwarteter und realisierter Rate größer ist. Man kann hier von einer Schocktherapie der Zentralbank sprechen, die dann aber auch zu einer stärkeren Zunahme der Reputation führt als im dritten Fall. Im Optimalfall steigt die Reputation so weit, daß die Wirtschaftssubjekte sogar der neuen Ankündigung der Zentralbank glauben, die unterhalb der in der ersten Periode liegt. Die Politik ist wieder glaubwürdig. Die Zentralbank kann diese Rate dann realisieren, 
sich so weiter Reputation aufbauen und muß dafür keine Arbeitslosigkeit „produzieren". 17

Im fünften Fall glauben die Wirtschaftssubjekte der Ankündigung der Zentralbank, welche jedoch durch Realisation einer höheren Rate eine Überraschungsinflation produziert. Dadurch geht zwar kurzfristig die Arbeitslosigkeit zurück, andererseits verliert die Zentralbank so Reputation. Die Wirtschaftssubjekte passen ihre Erwartungen in der nächsten Periode nach oben an, und eine Antiinflationspolitik der Zentralbank ist nicht mehr glaubwürdig. Will die Zentralbank nun ihre Reputation steigern, muß sie eine Überraschungsdeflation herbeiführen mit den damit verbundenen Kosten.

Im Gegensatz zum BG-Modell führt hier ausschließlich die Realisation geringer Inflationsraten zum Aufbau von Reputation. Eine glaubwürdige Geldpolitik wird nur über eine Antiinflationspolitik erzielt, nicht schon dadurch, daß die Erwartungen der Wirtschaftssubjekte erfüllt werden. Bestätigt die Zentralbank die Wirtschaftssubjekte in ihrer Meinung, daß die Geldpolitik glaubwürdig ist, indem sie ihre eigene Ankündigung einhält, führt dies zum Aufbau von Reputation. Aber auch wenn die Wirtschaftssubjekte die Ankündigung für nicht glaubwürdig halten, kann die Zentralbank ihre Reputation steigern, indem sie eine Inflationsrate realisiert, die unterhalb der Erwartungen liegt. Sie muß dabei nicht unbedingt ihre eigene Ankündigung einhalten. Je näher ihre realisierte Inflationsrate allerdings an die Ankündigung heranreicht, um so größer wird der positive Effekt auf die Reputation. Andererseits wird die Zentralbank hier auch bedenken, daß sie ihre Reputation wegen der dazu notwendigen Überraschungsdeflation mit einer Stabilisierungskrise „bezahlen“ muß. Je dichter ihre Realisation an der erwarteten Rate liegt, desto geringer ist die resultierende Arbeitslosigkeit, aber auch die Reputation. Andererseits führt die Reputation in der nächsten Periode zu geringeren Inflationserwartungen, die Geldpolitik wird glaubwürdiger und der für die Reputation zu zahlende Preis in Form der Arbeitslosigkeit kann zurückgehen.

Im BG-Modell ist die Reputation mit einem positiven Ansehen gleichgesetzt, d.h. es wird implizit unterstellt, daß das Ansehen einer Zentralbank bei den Wirtschaftssubjekten um so höher ist, je stärker sie die Inflation bekämpft, d.h. die Wirtschaftssubjekte präferieren immer die Inflationsvermeidung. Genau dies wird im Rahmen dieser Arbeit aber nicht unterstellt. Es wird deshalb zwischen den beiden Begriffen Prestige und Reputation unterschieden, die im all-

17 Diese Interpretation entspricht auch den Ergebnissen von Fellner (1976) und Haberler (1980). Danach werden die negativen wirtschaftlichen Effekte eines Antiinflationsprogramms um so länger und heftiger ausfallen, je unglaubwürdiger es ist. 
gemeinen Sprachgebrauch zwar synonym verwendet werden, im Rahmen der Geldtheorie aber etwas Unterschiedliches aussagen.

Prestige wird hier im Sinne von Ansehen bzw. Beliebtheit bei der Bevölkerung interpretiert. Es handelt sich somit um einen unbestimmten Begriff, da Prestige auf unterschiedliche Weise aufgebaut werden kann, und zwar je nach Grad der Inflationsaversion der Bevölkerung: Bei einer inflationsaversen Bevölkerung erhält eine Zentralbank Prestige durch eine Antiinflationspolitik und verliert es durch eine „Inflationspolitik“. Bei einer weniger inflationsaversen Bevölkerung dagegen erhält eine Zentralbank Prestige, indem sie das Stabilitätsziel eher vernachlässigt und bei der Verfolgung anderer Ziele, z.B. der Vollbeschäftigung, höhere Inflationsraten zuläßt.

Reputation dagegen kann eine Zentralbank nur erlangen, wenn sie als inflationsavers gilt, unabhängig von den Präferenzen der Bevölkerung. Reputation ist aus wissenschaftlicher Sicht immer positiv zu beurteilen ${ }^{18}$, aus Sicht der Bevölkerung kann sie aber positiv oder negativ bewertet werden. Im letzteren Falle erkennt die Bevölkerung nicht die Gefahren einer Inflationierung und fragt dementsprechend nicht im ausreichenden Maße Stabilität nach. Sie ist im Gegenteil zu einem Verzicht auf eine (strikte) Antiinflationspolitik bereit, damit andere, in ihren Augen wichtigere Ziele realisiert werden können. In diesem Fall ist eine Politik, die höhere Inflationsraten in Kauf nimmt, zwar dem Prestige, nicht aber der Reputation förderlich. Umgekehrt führt in dieser Situation eine Antiinflationspolitik zwar zum Aufbau von Reputation, mindert aber das Prestige der Zentralbank. Die Erzielung von Prestige über den Aufbau von Reputation, d.h. eine strikte Antiinflationspolitik, ist damit nur ein Spezialfall und zwar bei inflationsaverser Bevölkerung.

Um nun den Begriff Prestige zu integrieren, wird zunächst vereinfachend unterstellt, daß die Wirtschaftssubjekte immer die Rate erwarten, die sie sich von der Zentralbank tatsächlich wünschen. ${ }^{19}$ Bei dieser Annahme im BG-Modell bringt jede glaubwürdige Politik der Zentralbank nicht nur Reputation, sondern

18 Dies ergibt sich aus den für die Gesellschaft insgesamt primär negativen Effekten einer Inflation. Vgl. hierzu die umfangreiche Literatur zu den Inflationswirkungen, z.B. Issing (1990), S. 191-217, Pohl (1981), S.133-218 oder den eher formalen Ansatz von Heubes (1989), S. 125-144.

19 Diese vereinfachende Annahme wird hier nur getroffen, um den Begriff Prestige zu veranschaulichen. Tatsächlich sind die Erwartungen und Wünsche der Bevölkerung als unabhängig voneinander zu sehen. Die Erwartungen werden bestimmt durch die Meinung, was die Zentralbank aus Sicht der Bevölkerung realisieren wird, während die Wünsche der Wirtschaftssubjekte durch die eigene Bewertung der Politik bestimmt werden. 
auch Prestige, da die Zentralbank die Erwartungen, also die Wünsche der Bevölkerung, realisiert (vgl. Abbildung 2.3). Diese Interpretation ist jedoch unrealistisch, wie am zweiten Fall des BG-Modells deutlich wird. Die Wirtschaftssubjekte erwarten hier die schlechteste Inflationsrate, wünschen sich diese also von der Zentralbank, welche dann tatsächlich realisiert wird. Diese Politik bringt der Zentralbank nicht nur Prestige bei der Bevölkerung ein, sondern auch Reputation, so daß die Wirtschaftssubjekte für die nächste Periode die beste Inflationsrate erwarten.

Abbildung 2.3: Reputationsaufbau bei Glaubwürdigkeit (BG-Modell)

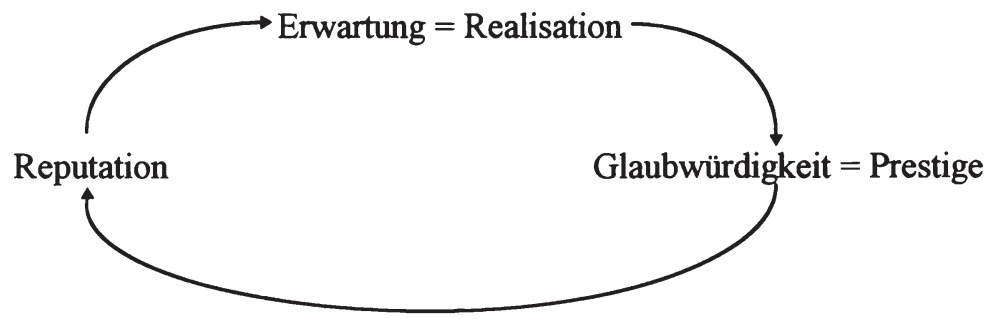

Realistischer ist die Interpretation der Modifikation, in der zwischen Glaubwürdigkeit und Prestige differenziert wird. Nur im ersten Fall ist die Geldpolitik glaubwürdig, führt zur Reputation und zu Prestige, da die Wirtschaftssubjekte sich auch geringe Inflationsraten von der Zentralbank wünschen, die diese dann auch durchführt (vgl. Abbildung 2.5, äußerer Pfad). Im zweiten Fall ist die Politik zwar nicht glaubwürdig $\left(\Pi^{\mathrm{a}} \neq \Pi^{\mathrm{e}}\right)$, bringt der Zentralbank aber trotzdem Prestige ein, weil sie das tut, was die Bevölkerung sich wünscht $\left(\Pi^{\mathrm{e}}=\Pi^{\mathrm{ZB}}\right)$. Da die Wirtschaftssubjekte sich aber keine Antiinflationspolitik wünschen, erlangt die Zentralbank durch den Aufbau von Prestige hier keine Reputation (vgl. Abbildung 2.5, innerer Pfad). Anders ist dies im dritten Fall, wo die Politik wieder unglaubwürdig ist, die Zentralbank sich aber Reputation aufbaut, indem sie eine geringere als die erwartete Rate realisiert, dafür aber auf Prestige verzichtet. Gleiches gilt für den vierten Fall (vgl. Abbildung 2.4, linke Seite).

Die Modifikation macht deutlich, daß die Erzielung von Prestige über den Aufbau von Reputation immer ein Spezialfall ist. Nur wenn die Bevölkerung inflationsavers ist, sind Reputation und Prestige synonym verwendbar. Sobald der Bevölkerung das Stabilitätsbewußtsein aber fehlt, müssen beide Begriffe voneinander abgegrenzt werden, was in der nun folgenden modifizierten Version von $B G$ berücksichtigt wird. 
Abbildung 2.4: Reputationsaufbau bei Glaubwürdigkeit und Unglaubwürdigkeit (modifiziertes Modell)

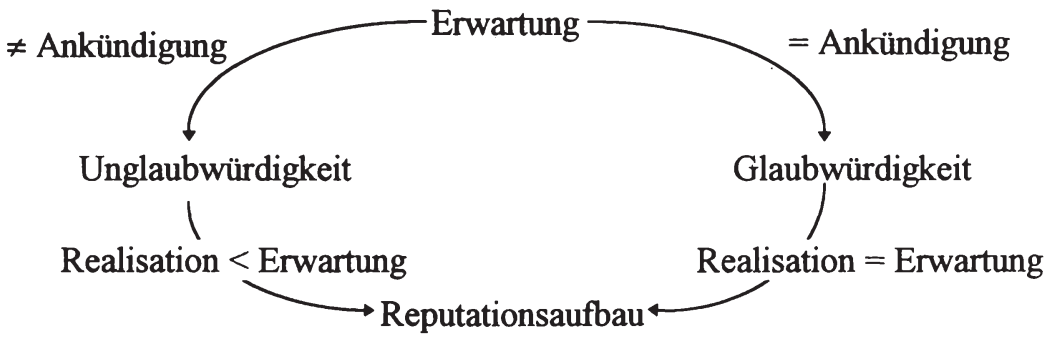

Abbildung 2.5: Prestige und Reputation (modifiziertes Modell)

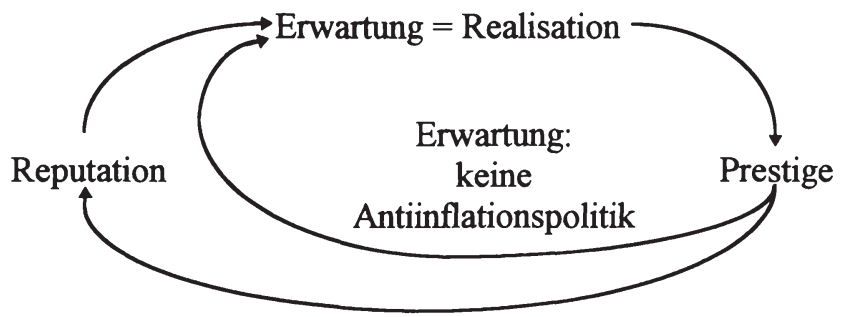

Erwartung: Antiinflationspolitik

\subsection{Das modifizierte Barro/Gordon-Modell}

Wie im Grundmodell von BG gilt auch hier, daß die als zu hoch betrachtete Arbeitslosenrate mittels einer Überraschungsinflation kurzfristig gesenkt werden kann.

Verzichtet wird hier jedoch auf die Annahme symmetrischer Informationen, d.h. Zentralbank und Öffentlichkeit verfügen nun nicht mehr über die gleichen Kenntnisse. ${ }^{20}$ Die Wirtschaftssubjekte müssen nicht die wahren Neigungen der Zentralbank kennen, letztere hat diesbezüglich einen Informationsvorsprung. Die Wirtschaftssubjekte haben lediglich eine Vorstellung darüber, wie sie die Zentralbank einschätzen, und diese Einschätzung kann die Zentralbank durch eine ,gute“ oder ,schlechte“ Politik beeinflussen.

20 Insbesondere diese Annahme bei BG wurde heftig kritisiert. Vgl. z.B. Blackburn/ Christensen (1989) und Cukierman (1986). 
Zudem wird die Inflationsrate nicht mehr als Strategievariable der Zentralbank angenommen. Sie kann sie nur noch beeinflussen, z.B. über die Geldmengensteuerung, aber nicht mehr allein setzen, da noch weitere Effekte auf die Inflation wirken. Die Strategievariable der Wirtschaftssubjekte bleibt weiterhin die Inflationserwartung.

Bisher wurde unterstellt, daß nur eine einzige Autorität die gesamte Wirtschaftspolitik bestimmt. ${ }^{21}$ Geht man jedoch davon aus, daß zwei Autoritäten diese Aufgabe erfüllen, indem die Politiker für die Fiskalpolitik und die Zentralbank, je nach Status mehr oder weniger, für die Geldpolitik zuständig ist, muß man den beschriebenen Ansatz insofern modifizieren, als die Inflationsrate nun nicht mehr von einem Akteur allein gesetzt werden kann, sondern durch alle Akteure der Wirtschaftspolitik gemeinsam bestimmt wird:

$$
\Pi=\Pi_{\mathrm{GP}}+\Pi_{\mathrm{FP}}+\Pi_{\mathrm{R}} \quad \text { mit: }
$$

$$
\Pi_{\mathrm{GP}}=\gamma \cdot \Pi_{\mathrm{ZB}}+(1-\gamma) \cdot \Pi_{\mathrm{P}} \quad \text { es gilt: } 0 \leq \gamma \leq 1
$$

Die tatsächlich realisierte Inflationsrate (П) wird jetzt nicht nur durch die Geldpolitik $\left(\Pi_{\mathrm{GP}}\right)$, sondern auch von der Fiskalpolitik $\left(\Pi_{\mathrm{FP}}\right)$ bestimmt. Zusätzlich werden unter der Restgröße $\Pi_{R}$ sämtliche anderen Faktoren zusammenge$\mathrm{fa} B \mathrm{t}$, die die Inflationsrate noch beeinflussen. Hierbei ist insbesondere an den Einfluß der Tarifparteien zu denken, deren Lohnpolitik zur Steigerung der Inflationsrate beitragen kann, wenn Löhne ausgehandelt werden, die über der Produktivität liegen. Übersteigt die Produktivitätsentwicklung hingegen die ausgehandelten Lohnsteigerungen, hat die Lohnpolitik einen deflatorischen Effekt. Sie ist inflationsneutral, wenn beide Größen sich gleich entwickeln. Dieser Einfluß der Tarifparteien gilt unter der Annahme, daß Lohnabschlüsse sich sofort in den Preisen widerspiegeln, während Mengenanpassungen am Arbeitsmarkt, z.B. in Form von Entlassungen, erst später realisiert werden können.

Neben der Lohnpolitik beeinflussen aber auch Wechselkursentwicklungen oder externe reale Schocks sowie ähnliches mehr die Inflationsrate. Diese Entwicklungen sind von der Zentralbank nicht zu kontrollieren und werden deshalb als Restgröße zusammengefaßt. Gleiches gilt für die inflatorischen Effekte der von der Regierung gesteuerten Fiskalpolitik. Auf diese kann die Zentralbank lediglich durch Appelle einwirken. Andererseits sind hier auch die Kreditaufnahmemöglichkeiten der Regierung bei der Zentralbank von Bedeutung. Aufgrund der geringen Einflußmöglichkeit der Zentralbank auf die beiden Größen $\Pi_{F P}$

Die Zentralbank war nur als ausfuhrendes Organ der Regierung zu sehen. 
und $\Pi_{R}$ wird ihre Summe als unvermeidbare Inflationsrate zusammengefaßt, die mit $2 \%$ angesetzt wird. ${ }^{22}$ Eine Inflationsrate in dieser Höhe lasten auch die Wirtschaftssubjekte nicht der Zentralbank an, da sie die Zusammensetzung der Inflationsrate in ihren Erwartungen berücksichtigen. In die Reputation der Zentralbank fließt diese unvermeidbare Inflationsrate also nicht ein, sondern nur der Teil, den sie auch verursacht hat. Sollte die von der Zentralbank nicht verursachte Inflationsrate $2 \%$ übersteigen, muß die Zentralbank über eine entsprechende Öffentlichkeitsarbeit versuchen, den Wirtschaftssubjekten dies zu verdeutlichen, damit sie ihre Reputation nicht „ungerechtfertigterweise“ verliert.

Verantwortlich ist die Zentralbank für die Realisation der von der Geldpolitik verursachten Inflationsrate. Allerdings ist hierbei wiederum der Einfluß der Regierung auf die Geldpolitik zu berücksichtigen, was in Gleichung (2.18) zum Ausdruck gebracht wird. Die Gewichtung der Teilinflationsraten entspricht den Machtverhältnissen, was durch den Parameter $\gamma$ ausgedrückt wird. Je größer $\gamma$ ist, desto mächtiger bzw. unabhängiger ist die Zentralbank. Es wird angenommen, da $B$ die Zentralbank immer eine geringere Inflationsrate setzt als die Regierung. Diese Annahme erscheint realistisch, wenn man berücksichtigt, daß die Regierung starken Anreizen zur Inflationierung unterliegt, wie sich später noch zeigen wird. Über die Anreize der Zentralbank kann man hingegen ohne weitere Annahmen noch keine Aussagen treffen. ${ }^{23}$

Diese Annahme berücksichtigen auch die Wirtschaftssubjekte bei der Bildung ihrer Erwartungen:

$$
\Pi^{\mathrm{e}}=\gamma \cdot \Pi_{Z B}^{\mathrm{e}}+(1-\gamma) \cdot \Pi_{\mathrm{P}}^{\mathrm{e}}+2 \% \quad \text { mit: } \Pi_{Z B}^{\mathrm{e}}<\Pi_{\mathrm{P}}^{\mathrm{e}}
$$

Die Erwartungen sind um so geringer, je größer der Einfluß der Zentralbank auf die Inflationsrate ist. Der Machtparameter $\gamma$ stellt für die Wirtschaftssubjekte allerdings keine sichere Variable dar, weil sie nur Vermutungen über die Höhe von $\gamma$ anstellen können. Der Grund dafür ist darin zu sehen, daß $\gamma$ nicht ausschließlich von der rechtlichen Unabhängigkeit abhängt, die jedes Wirtschaftssubjekt im Gesetz nachlesen kann, sondern auch von der informellen Macht, die nur durch Beobachtung zu ermitteln ist. Allerdings hängt diese informelle Macht von so vielen Faktoren ab, daß sie für die Wirtschaftssubjekte nur schwer einschätzbar ist.

22 Diesen Wert verwendet auch die Bundesbank zur Berechnung ihrer Geldmengenziele.

$23 \mathrm{Zu}$ den Anreizen für die Regierung vgl. Kapitel 3.1 und 3.2. Die Anreize für die Zentralbank werden im Kapitel 3.3 analysiert. 
Die Höhe der Inflationserwartungen wird im Ausgangsmodell und hier als Indikator für die Reputation betrachtet. Allerdings wird die Reputation der Zentralbank hier nur von dem Teil der Inflationsrate bestimmt, der durch die Geldpolitik verursacht wird $\left(\Pi_{\mathrm{GP}}\right)$. Die Höhe der Erwartung bezüglich dieser Größe spiegelt also die Reputation der Zentralbank wider. Während die Reputation im Basismodell eine objektive Größe darstellt, weil die Wirtschaftssubjekte die für die Reputation relevanten Präferenzen der Zentralbank kennen, handelt es sich bei der Reputation in der modifizierten Version um eine subjektive Größe, da die Wirtschaftssubjekte ihre Inflationserwartungen nicht mehr in Abhängigkeit der tatsächlichen Präferenzen der Zentralbank wählen. Mit Hilfe aller ihnen zugänglichen Informationen, wie den vergangenen Realisationen, dem juristischen Rahmen für die Zentralbank usw., stellen die Wirtschaftssubjekte Schätzungen für die die Reputation bestimmenden Größen an. Insbesondere müssen die Wirtschaftssubjekte sich eine Meinung bilden über die Stabilitätsvorliebe der Zentralbank und deren Chance, diese auch tatsächlich zu realisieren. Aufgrund dieser Einschätzung, die nicht korrekt sein muß, bilden die Wirtschaftssubjekte ihre Inflationserwartungen bezüglich der von der Zentralbank festzulegenden Inflationsrate, deren Höhe in Verbindung mit der Ankündigung der Zentralbank die Glaubwürdigkeit der Geldpolitik bestimmt. Definitionsgemäß wird die Glaubwürdigkeit als Differenz zwischen Ankündigung einer Politik und den Erwartungen der Wirtschaftssubjekte bezüglich der Realisierung dieser Ankündigung verstanden.

Anhand dieser Definition wird auch der Unterschied zwischen den beiden Begriffen Reputation und Glaubwürdigkeit deutlich. ${ }^{24}$ Eine antiinflationäre Geldpolitik kann glaubwürdig sein, selbst wenn eine $10 \%$ ige Inflationsrate erwartet wird, wenn sie angekündigt wurde. Bedingung ist nur, daß die in der Vorperiode realisierte Rate über der gegenwärtigen Ankündigung lag. Andererseits zeigt eine erwartete Inflationsrate in dieser Höhe, daß die Zentralbank sich noch nicht den Ruf erwerben konnte, ein Inflationsgegner zu sein. Sie besitzt also noch keine bzw. noch keine hohe Reputation. Nach und nach kann die Zentralbank sich aber über eine glaubwürdige Geldpolitik Reputation aufbauen. Da annahmegemäß die Ankündigungen von Periode zu Periode geringer ausfallen müssen, liegt eine glaubwürdige Politik nur vor, wenn auch die Inflationserwartungen ständig zurückgehen. Dafür werden die Wirtschaftssubjekte sich entscheiden, wenn die Zentralbank ihre Erwartungen nicht enttäuscht, also in jeder Periode ihre Ankündigung auch realisiert. 
Die Zielfunktion der Zentralbank, die zur Bestimmung der Reputation dient, ist die gleiche wie im Ausgangsmodell:

$$
Z_{t}=E\left[z_{t}+q \cdot z_{t+1}\right] ; \text { auch hier gilt: }
$$

$$
\mathrm{z}_{\mathrm{t}}=\frac{\mathrm{a}}{2} \cdot \Pi_{\mathrm{t}}^{2}-\mathrm{b} \cdot\left(\Pi_{\mathrm{t}}-\Pi_{\mathrm{t}}^{\mathrm{e}}\right) \text { mit: } 0 \leq \mathrm{q} \leq 1 \text { und } 0 \leq \mathrm{a}, \mathrm{b} \leq 1 \text { und } \mathrm{a}+\mathrm{b}=1
$$

Die Zentralbank bewertet die Inflation negativ, während eine Überraschungsinflation wegen ihrer kurzfristig positiven Wirkung auf die Beschäftigung einen Nutzen stiftet. Wieder hängen Kosten und Nutzen von den Zielgewichtungen a und $\mathrm{b}$ ab, und $\mathrm{q}$ drückt den Diskontierungsfaktor aus. Die Größen $\mathrm{a}, \mathrm{b}$ und $\mathrm{q}$ sind von den Präferenzen der Zentralbank abhängig. Diese stellen für die Zentralbank private Informationen dar, weil die Wirtschaftssubjekte sie nicht kennen, also nur unvollkommene Informationen besitzen. Dafür kennen sie auch hier die Form der Kostenfunktion, wie die Zentralbank sie nutzt. Auf die Werte für die Parameter schließen sie durch Beobachtung und Berücksichtigung der Rahmenbedingungen, unter denen die Zentralbank wirken muß.

Zur Bestimmung ihrer Erwartungen minimieren die Wirtschaftssubjekte die Kostenfunktion der Zentralbank, berücksichtigen dabei aber ihre Schätzungen für die Präferenzen der Zentralbank. Die Zentralbank minimiert dann ihrerseits ihre Kostenfunktion, in der sie die tatsächlichen Werte für ihre Neigungen berücksichtigt. Die Inflationserwartungen der Wirtschaftssubjekte sind ihr dabei bekannt, da sie vorher bestimmt wurden.

$\mathrm{Zu}$ Beginn der Periode kündigt die Zentralbank aufgrund ihrer Präferenzen (entsprechend der BG-Formel für die optimale Inflationsrate, Gleichung (2.15)) ihre beabsichtigte Inflationsrate an. Realisiert sie diese, die im Falle einer glaubwürdigen Geldpolitik auch erwartet wurde, bringt ihr dies Reputation ein, die sich darin äußert, daß die Wirtschaftssubjekte ihre Erwartungen für die nächste Periode senken.

Glauben die Wirtschaftssubjekte jedoch der Ankündigung der Zentralbank nicht, so daß ihre Geldpolitik unglaubwürdig ist, kann die Zentralbank sich nur Reputation aufbauen, wenn sie die Erwartungen der Wirtschaftssubjekte gerade nicht erfüllt. Nur die Realisation einer Inflationsrate, die unterhalb der Erwartungen liegt, führt nun zum Aufbau von Reputation. Dabei wird die Reputation um so größer ausfallen, je näher die Realisation der Zentralbank an ihrer Ankündigung liegt. Andererseits bedeutet eine zunehmende Differenz zwischen erwarteter und durchgeführter Inflationsrate auch eine Steigerung der Kosten für die Antiinflationspolitik, da die Stabilisierungskrise um so größer 
ausfallen muß. Eine hohe Reputation ermöglicht der Zentralbank also die Durchführung einer stabilitätsorientierten Geldpolitik, ohne der Gesellschaft dafür Kosten in Form von Arbeitslosigkeit aufzuerlegen.

Anders als bei BG sind hier unterschiedliche Grade der Reputation denkbar, was der folgende Erwartungsmechanismus zum Ausdruck bringt:

$$
\Pi^{\mathrm{e}}= \begin{cases}\Pi^{*}=\Pi^{\text {optimal }}(\text { opt })=0 & \text { bei } 100 \% \text { Reputation } \\ 0<\Pi^{*} \leq \dot{\Pi}^{\mathrm{e}}<\hat{\Pi} & \text { Reputation zwischen } 0 \text { und } 100 \% \\ \hat{\Pi}=\frac{\mathrm{b}}{\mathrm{a}} & \text { ohne Reputation }\end{cases}
$$

Bei einer Reputation der Zentralbank von $100 \%$ gehen die Wirtschaftssubjekte davon aus, daß die Zentralbank ein absoluter Inflationsgegner ist und den Teil der Inflationsrate, den sie kontrollieren kann, mit Null festlegt. Da die Wirtschaftssubjekte aber auch wissen, daß in die tatsächliche Inflationsrate eine Restgröße von $2 \%$ eingeht, berücksichtigen sie dies in ihren Erwartungen. Für die Reputation ist aber ausschließlich die Inflationsrate wichtig, die der Zentralbank ,,angelastet" wird, weshalb nur diese im weiteren Verlauf zu berücksichtigen ist. Die schlechteste Inflationsrate (b/a) wird nur erwartet, wenn die Zentralbank überhaupt keine Reputation besitzt. Sobald sie aber eine Reputation zwischen 0 und $100 \%$ genießt, wie es am realistischsten ist, erwarten die Wirtschaftssubjekte eine Inflationsrate, die zwischen beiden Extremen liegt. Vorher waren nur beide Extremfälle möglich, d.h. die Zentralbank besaß Reputation oder nicht.

Allerdings muß der Fall einer positiven, aber nicht perfekten Reputation weiter differenziert werden, da sowohl eine glaubwürdige als auch eine unglaubwürdige Geldpolitik vorliegen kann, je nachdem, ob die angekündigte Inflationsrate erwartet wird:

$$
\Pi^{\mathrm{e}}=\left\{\begin{array}{l}
0<\Pi^{\mathrm{opt}}=\Pi^{\mathrm{a}}=\dot{\Pi}^{\mathrm{e}} \text { glaubwürdige Politik } \\
0<\Pi^{\text {opt }}=\Pi^{\mathrm{a}}<\dot{\Pi}^{\mathrm{e}} \text { unglaubwürdige Politik }
\end{array}\right.
$$

Zu Beginn jeder Periode kündigt die Zentralbank aufgrund ihrer Präferenzen ihre optimale Inflationsrate an, die sie entsprechend der BG-Formel für $\Pi^{\text {opt }}$ berechnet. Zwar kennen die Wirtschaftssubjekte die Berechnungsmethode für $\Pi^{\text {opt }}$, da sie aber nicht die wahren Präferenzen der Zentralbank kennen, können sie eine andere Inflationsrate erwarten, als die Zentralbank sie ankündigt. Für diesen Fall einer unglaubwürdigen Geldpolitik ist für die Zentralbank die 
Realisation ihrer Ankündigung nicht mehr optimal. Sie muß dann eine „neue“ optimale Inflationsrate berechnen, in der sie nun auch die Inflationserwartungen berücksichtigen muß. Je nach Höhe der Inflationserwartungen wird die „neue“ optimale Rate variieren. Nun bestimmt also nicht mehr ausschließlich die Zentralbank über die optimale Inflationsrate, indem sie ihre Präferenzen berücksichtigt, wie dies im BG-Modell der Fall war. Die Zentralbank wird bei der Wahl ihrer optimalen Rate von den Wirtschaftssubjekten durch deren Rate restringiert. Diese Erwartungen jedoch spiegeln die Reputation der Zentralbank wider und können demnach von dieser durch ihre eigene Leistung beeinflußt werden. Gelingt es der Zentralbank, durch die Realisation einer Inflationsrate, die unterhalb der Erwartungen liegt, diese zu senken, kann sie somit auch die für sie optimale Inflationsrate von Periode zu Periode senken. Dies war bei BG nicht der Fall, weil es bei gegebenen Präferenzen der Zentralbank nur eine optimale Inflationsrate gab.

Glauben die Wirtschaftssubjekte hingegen die Ankündigung der Zentralbank, ist es für diese optimal, ihre Ankündigung einzuhalten. Dies entspricht dem BG-Fall, d.h. es gibt dann wieder nur eine optimale Inflationsrate. Mit der Wahl ihrer Inflationserwartungen bestimmen die Wirtschaftssubjekte also darüber, ob die Zentralbank ihre Ankündigung realisieren kann oder nicht. Es liegen sich selbst erfüllende Erwartungen vor, da die Zentralbank ihre Ankündigung realisiert, wenn die Wirtschaftssubjekte daran glauben bzw. andererseits die Ankündigung nicht realisiert werden kann, wenn die Wirtschaftssubjekte diese nicht erwarten.

BG untersuchen mit ihrem Modell nur einen möglichen Fall des Reputationsaufbaus. Sie gehen davon aus, daß Reputation immer durch die Realisation einer glaubwürdigen Geldpolitik aufgebaut wird. Wenn die Zentralbank die Inflationserwartungen erfüllt, erhält sie dafür Reputation, weil die Wirtschaftssubjekte ihre Erwartungen für die nächste Periode senken. Bei BG wird dann gleich wieder die „beste“ Inflationsrate erwartet. Da hier unterschiedliche Reputationsgrade möglich sind, werden die Inflationserwartungen nur langsam in Abhängigkeit von der Realisation der Zentralbank gesenkt. So kann die Zentralbank sich nach und nach Reputation aufbauen und sie ständig steigern.

Am Basismodell wird kritisiert, daß der Reputationsverlust bei einer schlechten Politik sowohl in seiner Höhe als auch in seiner Dauer genau bestimmt ist, ohne dabei die Höhe des Vergehens zu beachten. Jede realisierte Inflationsrate, die nicht den Erwartungen entspricht, führt dazu, daß sofort die ganze Reputation verloren geht, und dies genau für eine Periode. Die Wirtschaftssubjekte erwarten wieder die schlechteste Möglichkeit für die Inflationsrate. Die Zen- 
tralbank realisiert diese dann auch und erlangt so ihre komplette Reputation zurück, da sie die Erwartungen erfüllt hat.

Diese Annahme ist realitätsfern, da es unwahrscheinlich ist, daß mit einer schlechten Politik ein guter Ruf wiedererlangt werden kann. Im vorliegendem Erwartungsmechanismus wird bei jeder ,schlechten“ Politik die Inflationserwartung steigen, auch wenn sie erwartet war, und jede ,gute“ Politik läßt die Erwartungen sinken. Es gilt also:

$$
\Pi_{0}^{\mathrm{a}}<\Pi_{0}^{\mathrm{e}}=\Pi_{0}^{\mathrm{ZB}} \Rightarrow \Pi_{1}^{\mathrm{e}} \text { sinkt nicht } \Rightarrow \mathrm{R} \text { steigt nicht }
$$

falls aber:

$$
\Pi_{0}^{\mathrm{a}}<\Pi_{0}^{\mathrm{e}}>\Pi_{0}^{\mathrm{ZB}} \Rightarrow \Pi_{1}^{\mathrm{e}} \text { sinkt } \Rightarrow \mathrm{R} \text { steigt }
$$

Je öfter die Zentralbank hier eine gute Leistung hervorbringt, desto geringer werden die Inflationserwartungen, da die Reputation um so höher ist. Die Erfüllung der Erwartungen führt hier nur zur Reputationssteigerung, wenn die Wirtschaftssubjekte die Ankündigung der Zentralbank glauben $\left(\Pi^{\mathrm{a}}=\Pi^{\mathrm{e}}=\Pi^{2 \mathrm{~B}}\right.$ $\Rightarrow \mathrm{R} \uparrow$ ). Der Aufbau von Reputation erfordert in diesem Fall einer glaubwürdigen Geldpolitik auch keine Überraschungsdeflation. Die Zentralbank muß dann mit ihrer Antiinflationspolitik keine Arbeitslosigkeit verursachen, indem sie eine Inflationsrate setzt, die unterhalb der erwarteten liegt. Die Zentralbank realisiert ihre Ankündigung, die von den Wirtschaftssubjekten geglaubt wurde, und kann so von Periode zu Periode geringere Inflationsraten ankündigen, die für die Wirtschaftssubjekte dann glaubwürdig sind.

Denkbar ist jedoch auch der Fall, daß die Wirtschaftssubjekte die Ankündigung der Zentralbank nicht glauben, weil sie deren Präferenzen falsch einschätzen. Dies war bei BG nicht möglich, da diese Unsicherheit bezüglich der Präferenzen nicht gegeben war. Die Wirtschaftssubjekte waren deshalb in der Lage, die für die Zentralbank optimale Inflationsrate bei deren Präferenzen exakt zu bestimmen. Wird diese Sicherheit aufgegeben, muß auch der Fall einer unglaubwürdigen Geldpolitik für den Reputationsaufbau berücksichtigt werden. Die Wirtschaftssubjekte werden dann eine höhere Rate erwarten, als die Zentralbank sie aufgrund ihrer Präferenzen angekündigt hat $\left(\Pi^{\mathrm{e}}>\Pi^{\mathrm{a}}\right)$. Die vorher angekündigte Inflationsrate der Zentralbank ist nun nicht mehr optimal für sie, da sie jetzt auch die Inflationserwartungen der Wirtschaftssubjekte berücksichtigen muß. Die Realisation jeder Inflationsrate unterhalb der Erwar- 
tungen führt zu Kosten in Form von Arbeitslosigkeit ${ }^{25}$, welche bei der Ermittlung der optimalen Inflationsrate auch bedacht werden müssen.

Bevor nun die optimale Inflationsrate bei unglaubwürdiger Geldpolitik bestimmt wird, müssen noch die Variablen $\gamma, \mathrm{q}$, $\mathrm{a}$ und $\mathrm{b}$, wie sie in die Zielfunktionen eingehen, näher erklärt werden.

$\gamma$ als Machtparameter der Zentralbank wird um so größer sein, je unabhängiger eine Zentralbank ist, was wiederum eine um so geringere Einflußnahme der Regierung auf die Geldpolitik, ausgedrückt durch $1-\gamma$, impliziert. Diese Variable taucht in der Zielfunktion selbst zwar nicht mehr auf, bestimmt die Reputation aber wesentlich mit, indem sie entsprechend Gleichung (2.19) die Erwartungen mit beeinflußt. Andererseits bestimmt $\gamma$ auch die Richtung der Geldpolitik, indem alle übrigen Werte in Abhängigkeit von $\gamma$ gesetzt werden. Im Extremfall $\gamma=0$, d.h. im Falle einer vollkommen abhängigen Zentralbank, spielt es keine Rolle, ob die Zentralbank oder die Politiker direkt die Geldpolitik bestimmen, da die Zentralbank hier nur als ausführendes Organ zu betrachten ist. In diesem Fall werden die Wirtschaftssubjekte nicht die Präferenzen der Zentralbank berücksichtigen, sondern die der Politiker. Ist die Zentralbank hingegen unabhängig, wird sie andere Präferenzen haben als die Politiker, was die Wirtschaftssubjekte in ihrer Zielfunktion berücksichtigen. Die Reputation ist also in erheblichem Maße davon abhängig, wie stark die Zentralbank von der Politik abhängig ist.

Der Diskontierungsfaktor $\mathrm{q}=1 / 1+\mathrm{r}$ dient wieder als $\mathrm{Maß}$ für die Bedeutung, die der Zukunft beigemessen wird. Er ist um so höher, je wichtiger der Zentralbank die Zukunft ist. Im Extremfall nimmt er den Wert Eins an, was bedeutet, daß die Zentralbank die Zukunft als ebenso wichtig erachtet wie die Gegenwart und die zukünftigen Kosten deshalb schon heute voll berücksichtigt werden. Umgekehrt gilt, daß q um so geringer ist, je höher die Gegenwartsvorliebe ist, ausgedrückt durch die Zeitpräferenzrate $r$.

Da Politiker in der Regel eher kurzfristig denken, weil sie an ihrer Wiederwahl interessiert sind und deshalb primär die Wahltermine vor Augen haben, werden sie tendenziell eine hohe Zeitpräferenzrate haben. Im Gegensatz dazu werden unabhängige Zentralbanker eher langfristig denken, da sie nicht wahlabhängig sind und ihre Amtsperioden in der Regel über die Wahlzyklen hinausgehen. Es ist also realistisch, davon auszugehen, daß eine abhängige Zentralbank einen

25 Zugleich führt jede Realisation, die nicht den Erwartungen (hier im Sinne von Wünschen) entspricht, zum Verzicht auf Prestige zugunsten der Reputation. 
geringeren Diskontierungsfaktor wählt als eine unabhängige Zentralbank $\left(\mathrm{q}_{\mathrm{P}}<\mathrm{q}_{\mathrm{ZB}}\right)^{26}$. Auch die Annahme, daß sich $\mathrm{q}$ und a tendenziell in die gleiche Richtung bewegen, erscheint plausibel, da ein eher langfristig orientierter Zentralbanker auch a stärker gewichten wird als b, weil eine Überraschungsinflation die Arbeitslosigkeit nur kurzfristig senken kann.

Die beiden Parameter $a$ und $b$ stellen Gewichtungsfaktoren für die jeweiligen Ziele dar. Auch hier drückt a die Inflationsaversion aus. Der Faktor $b$ ist aber weiter zu interpretieren. Er kann wie bei BG als Vorliebe für eine Überraschungsinflation, mit der man zumindest kurzfristig die Beschäftigung steigern kann, gesehen werden, wenn unterstellt wird, daß die Zentralbank eine überraschend hohe Inflationsrate produziert, die einen Nutzen stiftet. Die mit b gewichtete Differenz zwischen $\Pi$ und $\Pi^{\mathrm{e}}$ kann aber auch zusätzliche Kosten einer Inflation bedeuten, wenn $\Pi$ kleiner als $\Pi^{\mathrm{e}}$ gewählt und eine Stabilisierungskrise hingenommen wird, um eine geringe Inflationsrate zu realisieren. Während im Basismodell für $\mathrm{a}$ und $\mathrm{b}$ jeder Wert größer Null zugelassen war, soll hier ihre Summe Eins ergeben, da nur zwei Ziele angestrebt werden und demzufolge die volle Präferenz auf beide Ziele verteilt werden muß.27

Die Wahl von a, und damit gleichzeitig von $b$, hängt nun davon $a b$, ob ein Politiker oder Zentralbanker diese wählt bzw. wie unabhängig ein Zentralbanker in seiner Entscheidung sein kann. Der Politiker wird bei seiner Überlegung die konjunkturelle Situation sowie den Wahltermin berücksichtigen. Ausgehend von der folgenden Matrix sind vier Fälle denkbar:

\begin{tabular}{|l|c|c|}
\hline Inflation & hoch & gering \\
\hline hoch & 1 & 2 \\
\hline gering & 3 & 4 \\
\hline
\end{tabular}

Diese vier Fälle sollen zunächst für einen Politiker betrachtet werden. Relativ einfach erscheint eine Entscheidung in den Fällen 2 und 3. Unabhängig vom Wahlzeitpunkt wird der Politiker im zweiten Fall seine Inflationsaversion a bzw. im dritten Fall seine Präferenz für die Beschäftigungssteigerung b stärker gewichten, da hier jeweils nur ein Ziel verletzt ist. Auch der vierte Fall er-

$\mathrm{q}_{\mathrm{p}}$ ist hier zu interpretieren als Wert, den eine abhängige Zentralbank setzt.

Diese Annahme führt dazu, daß man statt b auch 1-a schreiben könnte, worauf jedoch zunächst verzichtet wird, um die Vergleichbarkeit zum BG-Modell aufrechtzuerhalten. 
scheint nicht weiter problematisch, weil beide Ziele gut erfüllt sind und er deshalb beide gleich gewichten kann. Ein Politiker wird die Geldpolitik dazu nutzen, auch das Beschäftigungsziel zu realisieren, weshalb b hier die Überraschungsinflation gewichtet und somit einen positiven Nutzen aus einer Inflation beschreibt.

Der einzig problematische Fall ist der erste, da hier beide Ziele verfehlt sind. Die Entscheidung des Politikers wird in diesem Fall vom Wahltermin abhängen. Steht die Wahl kurz bevor, wird er b stärker gewichten, um die Arbeitslosigkeit zu senken. Da die Beschäftigung schneller reagiert als die Preise, die erst mit einer zeitlichen Verzögerung, nach Anpassung der Erwartungen, reagieren, wird zum Wahlzeitpunkt ein aus Sicht des Politikers optimales Ergebnis möglich. Er hat die Arbeitslosigkeit bekämpft, ohne dafür die Inflation noch weiter zu steigern, was erst nach der Wahl geschehen wird.

Ist andererseits der Wahlzeitpunkt noch in weiter Ferne, wird der Politiker zuerst die Inflation bekämpfen, also a stärker gewichten, um dann kurz vor der Wahl $b$ eine größere Bedeutung beimessen zu können und so wieder die Arbeitslosigkeit $\mathrm{zu}$ bekämpfen. Aufgrund der unterschiedlichen Reaktionsgeschwindigkeiten der beiden Ziele kann es im günstigsten Fall dazu kommen, $\mathrm{da} ß$ es dem Politiker genau zum Wahltermin gelungen ist, die Inflation und die Arbeitslosigkeit zu mindern, was seine Wiederwahlchance beträchtlich steigert. ${ }^{28}$

Die Gewichtung von $\mathrm{a}$ und $\mathrm{b}$ von seiten einer unabhängigen Zentralbank ist vom genauen Ausmaß der Unabhängigkeit, der Konfliktfreudigkeit mit der Regierung sowie der Zielfunktion der Zentralbank abhängig. Eine sehr unabhängige inflationsaverse Zentralbank, die einen Konflikt mit der Regierung nicht scheut und eventuell sogar die Öffentlichkeit hinter sich weiß, wird unabhängig von der konjunkturellen Situation und dem Wahltermin immer a stark gewichten und b sehr gering. Im Extremfall (der allerdings eher theoretischer Natur ist) wird sie nur noch versuchen, eine Inflation zu vermeiden, unabhängig davon, wie die wirtschaftliche Situation aussieht. Hier gewichtet $b$ eher eine Überraschungsdeflation, die einen zusätzlichen Kostenaspekt der Inflation darstellt, weil eine Stabilisierungskrise von der Zentralbank verursacht wird.

Je abhängiger eine Zentralbank ist, desto eher wird sie sich bei der Wahl ihrer Präferenzen wie ein Politiker verhalten. Gleiches gilt auch für eine zwar unabhängige Zentralbank, die aber weniger geneigt ist, sich mit der Regierung auseinanderzusetzen. Dies könnte z.B. der Fall sein, wenn die Zentralbanker sich 
bei der Regierung einen „Goodwill“" sichern wollen, etwa im Hinblick darauf, wie sie nach ihrer Zeit bei der Zentralbank weiterbeschäftigt werden.

Nachdem nun geklärt ist, wie die Werte für die Parameter $\mathrm{a}, \mathrm{b}$ und $\mathrm{q}$ festgelegt werden, soll die Reputation formal bestimmt werden. Wie im Grundmodell wird auch hier zur Vereinfachung von einem 2-Perioden-Modell ausgegangen. Zur Ermittlung der Reputation der Zentralbank dient die folgende Kostenfunktion als Grundlage 29 :

$$
Z_{t}=q(\gamma) \cdot\left[\frac{a(\gamma)}{2} \cdot \Pi_{t}^{2}-b(\gamma) \cdot\left(\Pi_{t}-\Pi_{t}^{e}\left(\gamma, \Pi_{t-1}\right)\right)\right]
$$

Auch hier kann ein Anreiz zur Inflation bestehen, weil so die Beschäftigung kurzfristig steigen könnte. Andererseits liegt aber auch ein Zwang zur Inflationsvermeidung vor, da durch Anpassung der Erwartungen in der nächsten Periode mit einer steigenden Inflation zu rechnen ist. Wieder fallen die Kosten erst in der Zukunft an, d.h. der Zwang zur Inflationsvermeidung muß mit q abdiskontiert werden. Der Anreiz besteht aus dem heutigen Gewinn.

Um den Zwang zur Stabilitätspolitik zu bestimmen, muß wieder die abdiskontierte Kostendifferenz berechnet werden, die sich ergibt aus den Kosten bei Realisierung der diskretionären Inflationsrate (dann besäße die Zentralbank überhaupt keine Reputation) und den Kosten, die bei Erzielung der optimalen Inflationsrate anfallen würden. Letzteres wäre der Fall der glaubwürdigen Geldpolitik. Wieder ergibt sich der Zwang zur Inflationsvermeidung wie folgt:

$$
E\left[q \cdot\left(\hat{z}_{t+1}-z_{t+1}^{*}\right)\right]=q \cdot\left(\frac{1}{2} \cdot \frac{b^{2}}{a}-\frac{a}{2} \cdot \Pi^{o p t 2}\right)=q \cdot \frac{a}{2} \cdot\left(\frac{b^{2}}{a^{2}}-\Pi^{o p t 2}\right)
$$

Anders sieht hier die Anreizfunktion aus. Der Anreiz äußert sich zwar wieder in der Kostendifferenz zwischen den Kosten bei Wahl der Inflationsrate bei Regelbindung und der bei Betrug, aber die Erträge für die Betrugslösung können hier geringer sein.

29 Dies ist die ausführliche Schreibweise. Sie zeigt die Abhängigkeit der Zielgewichtungen sowie des Diskontierungsfaktors und der Inflationserwartungen von der Macht der Zentralbank. Zusätzlich werden die Inflationserwartungen noch von der vergangenen Leistung bestimmt. Im weiterem Verlauf wird aber verkürzt nur noch $a, b$, q und $\Pi^{\mathrm{e}}$ geschrieben. Auch wird auf das tiefgestellte $\mathrm{t}$ verzichtet, da es sich immer um die gegenwärtige Periode handelt. Ist eine andere Periode gemeint, wird dies explizit herausgestellt. 
Im Basismodell wurde für die Betrugslösung unterstellt, daß die Zentralbank ankündigt, die optimale Inflationsrate zu realisieren, tatsächlich aber die diskretionäre Rate wählt, sobald die Wirtschaftssubjekte ihre Erwartungen entsprechend der Ankündigung gesetzt haben. Diese Lösung wird deshalb realisiert, weil in diesem Modell die Reputation entweder vorhanden ist oder nicht. In der Modellerweiterung ist auch eine Reputation zwischen 0 und 100\% denkbar. Die Wirtschaftssubjekte müssen deshalb nicht volles Vertrauen in die Zentralbank setzen, sondern können zwar eine bessere als die schlechteste Inflationsrate erwarten, aber auch nicht die für die Zentralbank optimale, selbst wenn sie diese angekündigt hat. Für die Betrugslösung ergibt sich deshalb bei einer unglaubwürdigen Geldpolitik der folgende Ertrag (ausgedrückt in Kosten):

mit: $\Pi=\frac{\mathrm{b}}{\mathrm{a}}$ und $\Pi^{\mathrm{e}}=\dot{\Pi}^{\mathrm{e}} \neq \Pi^{\mathrm{opt}}=\Pi^{\mathrm{a}}$

(2.23) $\widetilde{\mathrm{z}}=\frac{\mathrm{a}}{2} \cdot \frac{\mathrm{b}^{2}}{\mathrm{a}^{2}}-\mathrm{b} \cdot\left(\frac{\mathrm{b}}{\mathrm{a}}-\dot{\Pi}^{\mathrm{e}}\right)=-\frac{1}{2} \cdot \frac{\mathrm{b}^{2}}{\mathrm{a}}+\mathrm{b} \cdot \dot{\Pi}^{\mathrm{e}}$

Jetzt bestimmt nicht nur die realisierte Inflationsrate selbst den Ertrag, sondern auch die Inflationserwartungen, die ihn schmälern. Je größer aus Sicht der Wirtschaftssubjekte die Präferenz der Zentralbank für die Beschäftigungsankurbelung ist, desto kleiner wird der Ertrag der Inflation, da die Höhe der Überraschungsinflation um so geringer ist. Gleiches gilt bei hohen Inflationserwartungen, also einer wenig glaubwürdigen Antiinflationspolitik.

Um den kompletten Anreiz zur Inflation, also den Nutzen aus der Inflation, zu erhalten, muß wieder die Kostendifferenz zwischen den Kosten bei Erzielung der Inflationsrate bei Regelbindung und denen bei Vorliegen der Betrugslösung gebildet werden:

(2.24) $\mathrm{E}\left[\mathrm{z}^{*}-\widetilde{\mathrm{z}}\right]=\frac{\mathrm{a}}{2} \cdot \Pi^{\mathrm{opt} 2}-\left(-\frac{1}{2} \cdot \frac{\mathrm{b}^{2}}{\mathrm{a}}+\mathrm{b} \cdot \dot{\Pi}^{\mathrm{e}}\right)=\frac{\mathrm{a}}{2} \cdot\left(\frac{\mathrm{b}}{\mathrm{a}}-\Pi^{\mathrm{opt}}\right)^{2}+\mathrm{b} \cdot\left(\Pi^{\mathrm{opt}}-\dot{\Pi}^{\mathrm{e}}\right)$

Die Anreizfunktion in diesem Modell verläuft unterhalb der des Grundmodells, solange die Inflationserwartungen nicht der optimalen Rate, die angekündigt wurde, entsprechen, da der zweite Term dann negativ ist. Entsprechend dem Erwartungsmechanismus liegt $\dot{\Pi}^{\mathrm{e}}$ zwischen $\Pi^{\text {opt }}$ und der diskretionären Lösung $\hat{\Pi}=b / a$ und entspricht nur bei glaubwürdiger Geldpolitik der optimalen Inflationsrate $\Pi^{\mathrm{opt}}$. 
Abbildung 2.6: Abnehmender Anreiz zur Inflationierung bei einer Reputation zwischen 0 und $100 \%$

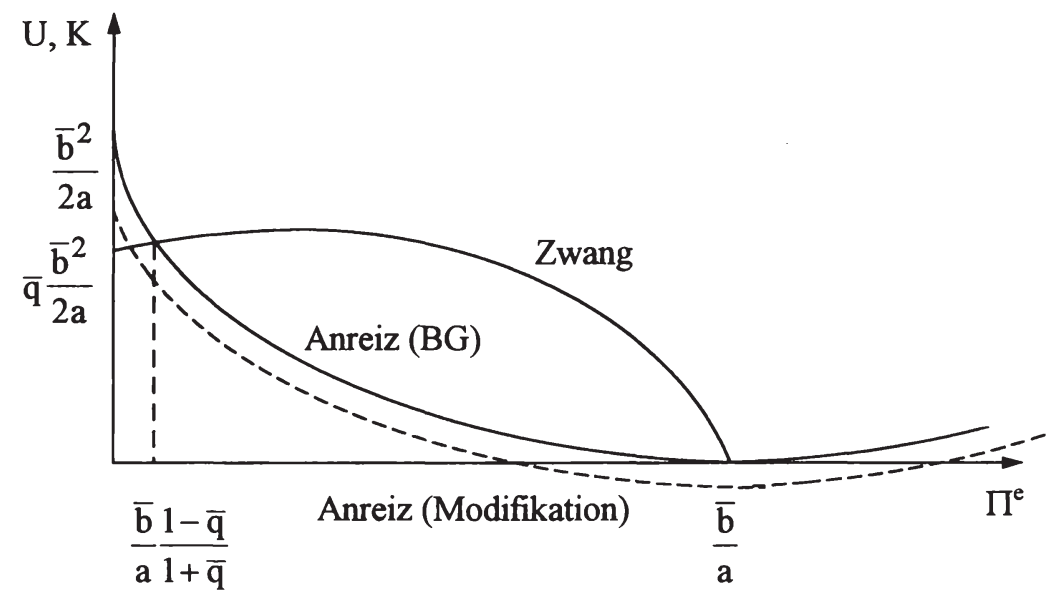

Bei gleichbleibender Zwangfunktion wird die optimale Inflationsrate bei unglaubwürdiger Geldpolitik in Richtung Ursprung verschoben, da der Nutzen einer Überraschungsinflation geringer wird. Andererseits muß berücksichtigt werden, daß die Realisation einer geringeren als der erwarteten Inflationsrate Kosten in Form einer Stabilisierungskrise verursacht, die bei der Berechnung der optimalen Inflationsrate zu berücksichtigen sind. Diese Kosten einer Anti-

Abbildung 2.7: Zunehmender Anreiz zur Inflationierung bei einer Reputation zwischen 0 und $100 \%$

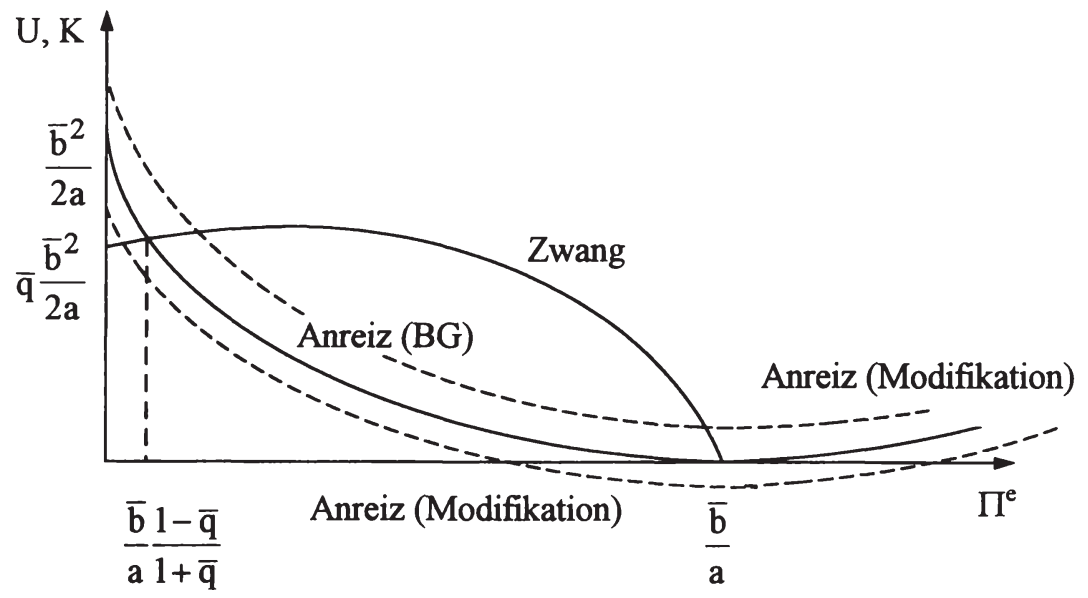


inflationspolitik stellen wiederum einen Anreiz zur Inflationierung dar, was eine Verlagerung der Kurve nach oben bedeutet.

Je nach Stärke der Verlagerung fällt bei unglaubwürdiger Geldpolitik die optimale Inflationsrate besser, schlechter oder genau so hoch aus wie im Fall von BG bei glaubwürdiger Politik. Zur exakten Bestimmung der optimalen Inflationsrate bei unglaubwürdiger Geldpolitik werden wieder die Anreiz- und Zwangfunktion gleichgesetzt:

$$
\mathrm{q} \cdot\left[\frac{\mathrm{a}}{2} \cdot\left(\frac{\mathrm{b}^{2}}{\mathrm{a}^{2}}-\Pi^{\mathrm{opt} 2}\right)\right]=\frac{\mathrm{a}}{2} \cdot\left(\frac{\mathrm{b}}{\mathrm{a}}-\Pi^{\mathrm{opt}}\right)^{2}+\mathrm{b} \cdot\left(\Pi^{\mathrm{opt}}-\dot{\Pi}^{\mathrm{e}}\right)
$$

(2.25) $\Pi^{\text {opt }}=\sqrt{\frac{2 \cdot b}{a \cdot(1+q)} \cdot \dot{\Pi}^{e}-\frac{1-q}{1+q} \cdot \frac{b^{2}}{a^{2}}}=\frac{b}{a} \cdot \sqrt{\frac{2 \cdot a}{b \cdot(1+q)} \cdot \dot{\Pi}^{e}-\frac{1-q}{1+q}}$

Damit für $\Pi^{\mathrm{opt}}$ eine Lösung existiert, darf der Wurzelausdruck nicht negativ werden. Die folgende Bedingung muß deshalb erfüllt $\operatorname{sein}^{30}$ :

(2.26) $\dot{\Pi}^{\mathrm{e}} \geq \frac{1}{2} \cdot \frac{\mathrm{b}}{\mathrm{a}} \cdot(1-\mathrm{q})$

Sobald die Inflationserwartungen genau diesem Wert entsprechen, liegt die Nullösung vor. Die Zentralbank kann dann eine Inflationsrate von Null realisieren. Im Basismodell ist dies nur der Fall, wenn $q=1$ oder $b=0$ gesetzt werden. Diese Möglichkeiten wurden aber von vornherein ausgeschlossen, da dies bedeuten würde, daß die Regierung entweder eine Zeitpräferenzrate von Null hätte oder ihr die Beschäftigung nicht wichtig wäre. Beide Fälle sind aber für eher kurzfristig denkende Politiker unrealistisch.

Im vorliegenden Fall sind beide Möglichkeiten durchaus denkbar, da man einer unabhängigen Zentralbank zutrauen kann, daß sie die Zukunft genauso stark berücksichtigt wie die Gegenwart $(\mathrm{q}=1)$ oder ausschließlich das Stabilitätsziel verfolgt $(a=1 \Rightarrow b=0)$. Zusätzlich ist die Nullinflation hier die optimale Lösung, wenn die Inflationserwartungen genau dem Wert aus Gleichung (2.26) entsprechen. Dies bedingt jedoch, daß die Wirtschaftssubjekte die Präferenzen der Zentralbank kennen bzw. richtig einschätzen. Solange die Zentralbank ihre maximale Reputation noch nicht erreicht hat, wird dieser Fall kaum eintreten. In der Regel werden die Inflationserwartungen höher ausfallen, als sie aufgrund

Im folgenden wird davon ausgegangen, daß diese Bedingung erfüllt ist. 
der tatsächlichen Präferenzen gerechtfertigt wären. Bei unglaubwürdiger Geldpolitik wird also eine Inflationsrate erwartet, die oberhalb der Ankündigung der Zentralbank liegt $\left(\Pi^{\mathrm{e}}>\Pi^{\mathrm{a}}\right)$.

Eine von der Zentralbank verursachte Inflationsrate von Null ist demnach wie bei BG ohne Stabilisierungskrise nur erreichbar, wenn die Zentralbank $b=0$ oder $\mathrm{q}=1$ setzt, was die Wirtschaftssubjekte zudem wissen müssen, also $\Pi^{\mathrm{e}}=0$ gilt. Kennen die Wirtschaftssubjekte die wahren Präferenzen der Zentralbank hingegen nicht, ist eine Nullinflation immer nur zu dem Preis einer Stabilisierungskrise erreichbar.

Die von der Zentralbank errechnete und realisierte optimale Inflationsrate ist hier jedoch nicht wie im BG-Modell die tatsächlich auch realisierte, da letztere sich entsprechend Gleichung (2.17) zusammensetzt. Demnach wird die tatsächliche Rate noch um einen Teil, den die Zentralbank nicht kontrollieren kann, nach oben „,verzerrt". Da dieser unkontrollierbare Teil aber mit $2 \%$ festgesetzt wurde, den auch die Wirtschaftssubjekte bei ihrer Erwartungsbildung und die Zentralbank bei ihrer Ankündigung berücksichtigen, kann aus der realisierten auf die von der Zentralbank verursachte Rate geschlossen werden. Anhand dieser Rate ziehen die Wirtschaftssubjekte dann Schlußfolgerungen bezüglich der Präferenzen der Zentralbank, die sie bei ihrer Erwartungsbildung berücksichtigen. ${ }^{31}$

Werden die so gebildeten Inflationserwartungen um 2\% gemindert, hat man die Höhe der Inflationserwartungen, die nur den von der Zentralbank zu verantwortenden Teil enthält und hier die Reputation zum Ausdruck bringt. Zur Festsetzung ihrer Erwartungen können die Wirtschaftssubjekte nur die tatsächlich realisierte Inflationsrate zur Hilfe nehmen, was ein Problem für die Zentralbank darstellen kann, wenn der von ihr nicht zu beeinflussende Inflationsanteil $2 \%$ übersteigt.

Selbst wenn eine vollkommen unabhängige Zentralbank $(\gamma=1)$ also eine Inflationsrate von Null realisieren sollte und die Lohnabschlüsse die Inflationsrate

31 Die Höhe der Erwartungen für die nächste Periode hängt also von der optimalen Inflationsrate dieser Periode ab. Die Bestrafung (Belohnung) in Form steigender (sinkender) Erwartungen erfolgt damit nicht unabhängig von der tatsächlichen „Erwartungsenttäuschung" der Wirtschaftssubjekte, wie dies bei BG der Fall war. Ein Modell, in dem die Größe der Abweichung zwischen Inflationserwartungen und tatsächlicher Realisation die Reaktion der Wirtschaftssubjekte bestimmt, haben Nowaihi/ Levine (1993) beschrieben. Sie gehen insbesondere darauf ein, daß es bei nur geringer Abweichung zu keinerlei Reaktion der Wirtschaftssubjekte kommt. 
nicht verändern sowie auch keine anderweitigen Verzerrungen vorliegen, kann es nicht zu einer tatsächlich realisierten Inflationsrate von Null kommen, solange die Fiskalpolitik inflationstreibend wirkt.

Gleichzeitig zeigt Gleichung (2.17), wie die Erwartungen hier gebildet werden. Im Gegensatz zu den anderen Modellen werden sie nicht direkt von der vergangenen Leistung der Akteure bestimmt, sondern nur indirekt. Aus den erbrachten Leistungen schließen die Wirtschaftssubjekte auf die Stabilitätsorientierung sowie die Zeitpräferenzrate der Autoritäten. ${ }^{32}$ Dabei werden die Wirtschaftssubjekte die Werte von a bzw. $\mathrm{q}$ aber nicht ausschließlich davon abhängig machen, für wie inflationsavers sie die Zentralbanker halten, sondern auch davon, ob diese ihre Inflationsaversion auch durchsetzen können, was durch $\gamma$ zum Ausdruck gebracht wird. Je geringer die tatsächliche Inflationsrate ist, desto höher wird $\gamma$ sein und desto höher wird der Wert für a angesetzt. Somit können die Zentralbanker auch hier durch ihr eigenes Handeln die Erwartungen beeinflussen, die ihrerseits die von der Zentralbank realisierte Inflationsrate beeinflußt und somit die Reputation der Zentralbank. Andererseits wird bei der Erwartungsbildung auch der institutionelle Rahmen berücksichtigt, der durch $\gamma$ zum Ausdruck kommt und von den Zentralbankern nicht beeinflußt werden kann. ${ }^{33}$

Eine weitere Interpretation der Erwartungen, insbesondere der Vergleich zum Basismodell, erfolgt später noch ausführlicher. Zunächst sollen die Bestimmungsgrößen der Erwartungen sowie der Reputation selbst näher untersucht werden.

Um die Wirkungsrichtung der zwei die Erwartungen beeinflussenden Variablen zu bestimmen ( $\mathrm{a}$ und q), muß die Erwartungsfunktion entsprechend abgeleitet werden. Zunächst aber muß sie noch mit der Hilfsvariablen $x>1$ erweitert werden, da in der Regel die Ungleichung $\dot{\Pi}^{\mathrm{e}}>\frac{1}{2} \cdot \frac{\mathrm{b}}{\mathrm{a}} \cdot(1-\mathrm{q})$ gelten wird:

Die Gewichtung der Vorliebe zur Beschäftigungsankurbelung muß nicht explizit bestimmt werden, da sie sich aus der Stabilitätsorientierung ergibt. Es gilt: $b=1-a$. Im weiteren Verlauf wird b so ausgedrückt.

33 So wird auch ein Zentralbanker, von dem die Öffentlichkeit weiß, daß er stabilitätsbewußt ist, keine hohe Reputation genießen, wenn ihm die Möglichkeit zur Durchsetzung seiner Präferenzen fehlt. Wenn er also nur in einer von der Regierung abhängigen Zentralbank wirken kann, werden die Wirtschaftssubjekte bei der Einschätzung über die potentielle Teilinflationsrate a tendenziell geringer gewichten, da dies tatsächlich von der Regierung dominiert wird. Die Reputation der Zentralbank wird also eher gering ausfallen. 


$$
\dot{\Pi}^{\mathrm{e}}=\frac{1-\mathrm{q}}{2} \cdot \mathbf{x} \cdot \frac{1-\mathrm{a}}{\mathrm{a}}
$$

$$
\frac{\partial \dot{\Pi}^{e}}{\partial a}=\frac{1-q}{2} \cdot x \cdot \frac{(-1) \cdot a-(1-a) \cdot 1}{a^{2}}=\frac{1-q}{2} \cdot x \cdot \frac{-a-1+a}{a^{2}}=\frac{1-q}{2} \cdot x \cdot \frac{-1}{a^{2}}<0
$$

Demnach nehmen die Inflationserwartungen mit steigender Inflationsaversion ab. Das heißt andererseits, daß sie steigen, wenn die Bevölkerung meint, einem Geldpolitiker gegenüberzustehen, dem die Beschäftigung wichtiger ist (b个 $\Rightarrow \dot{\Pi}^{\mathrm{e} \uparrow}$ ).

Analog ist die Wirkung von $\mathrm{q}$ auf $\dot{\Pi}^{\mathrm{e}}$ zu untersuchen. Es gilt:

$$
\frac{\partial \dot{\Pi}^{\mathrm{e}}}{\partial \mathrm{q}}=\frac{1-\mathrm{a}}{\mathrm{a}} \cdot \mathrm{x} \cdot \frac{1}{2} \cdot(-1)<0
$$

Je wichtiger dem Zentralbanker nach Meinung der Öffentlichkeit die Zukunft erscheint, desto geringer werden diese ihre Erwartungen setzen. Dies Ergebnis kann aber nicht überraschen, da sich a und $\mathrm{q}$ in die gleiche Richtung entwickeln.

Beide Ableitungen zeigen jedoch nur die Wirkung auf die Inflationserwartungen, wenn die Wirtschaftssubjekte meinen, daß ein Präferenzwandel stattgefunden hat. Interessant ist jedoch auch der Fall, daß ein Präferenzwandel tatsächlich vorliegt. Im Normalfall werden die Wirtschaftssubjekte dies anhand der von der Zentralbank realisierten Inflationsrate ablesen können. Denkbar ist aber auch, daß eine zunehmende Stabilitätsorientierung der Zentralbank von den Wirtschaftssubjekten nicht erkannt werden kann, weil der von der Zentralbank nicht kontrollierbare Teil der Inflationsrate in einer Periode höher ausfällt als ursprünglich angenommen. Es kann demnach sein, daß trotz größerer Anstrengung der Zentralbank bezüglich einer Antiinflationspolitik eine höhere Inflationsrate realisiert wird, weil ein exogener Schock zu einer größeren Restgröße führte. Nur wenn die Zentralbank den Wirtschaftssubjekten diese Entwicklung glaubhaft vermitteln kann, werden die Inflationserwartungen in der nächsten Periode nach unten angepaßt. Glauben die Wirtschaftssubjekte jedoch nicht an die „Schuld“ externer Faktoren, wird das tatsächlich gestiegene Stabilitätsbewußtsein der Zentralbank nicht durch sinkende Inflationserwartungen honoriert, sondern eventuell sogar mit steigenden Erwartungen quittiert. Die Wirkungsrichtung der Inflationserwartungen auf die Änderung der tatsächlichen Präferenz ist also nicht eindeutig bestimmbar. Die Ableitung der Inflati- 
onserwartungen nach der tatsächlichen Präferenz kann somit positiv, negativ oder Null werden. ${ }^{34}$

Um zu zeigen, wie die einzelnen Größen die Reputation beeinflussen, sind die Ableitungen von $\Pi^{\mathrm{ZB}}$ nach $\dot{\Pi}^{\mathrm{e}}$, a und q zu bilden.

$$
\Pi^{Z B}=\left[\frac{2 \cdot(1-a)}{a \cdot(1+q)} \cdot \dot{\Pi}^{e}-\frac{1-q}{1+q} \cdot \frac{(1-a)^{2}}{a^{2}}\right]^{\frac{1}{2}}
$$

Es gilt hierbei die Annahme, daß dieser Ausdruck nicht negativ werden soll. Da Reputation im Sinne von Stabilitätsorientierung definiert wird, führt die Realisation negativer Inflationsraten nicht zur Reputation. Eine perfekte Reputation liegt immer vor, wenn eine Nullinflation von der Zentralbank ,kostenlos" produziert werden kann. Leitet man nun die von der Zentralbank realisierte Inflationsrate nach den Inflationserwartungen $\mathrm{ab}$, ergibt sich der folgende Ausdruck:

$$
\frac{\partial \Pi^{Z B}}{\partial \dot{\Pi}^{e}}=\frac{1}{2} \cdot\left[\frac{2 \cdot(1-a)}{a \cdot(1+q)} \cdot \dot{\Pi}^{e}-\frac{1-q}{1+q} \cdot \frac{(1-a)^{2}}{a^{2}}\right]^{-\frac{1}{2}} \cdot \frac{2 \cdot(1-a)}{a \cdot(1+q)}>0
$$

Die Vorzeichenanalyse ergibt, da $ß$ mit steigenden Inflationserwartungen auch die Inflationsrate steigt, da der erste Term annahmegemäß positiv ist und bei Ausschluß der Extremlösung a $<1$ gilt, womit auch der zweite Term positiv wird. Geringe Inflationserwartungen ermöglichen also die Realisation einer geringen Inflationsrate, welche ihrerseits zum Reputationsaufbau (R) beiträgt, da gilt:

$$
\Pi^{\mathrm{e}} \downarrow \Rightarrow \Pi^{\mathrm{ZB}} \downarrow \Rightarrow \mathrm{R} \uparrow
$$

Anders sieht die Wirkungsrichtung der beiden Variablen a und $\mathrm{q}$ auf $\Pi^{\mathrm{ZB}}$ aus, wie mit den folgenden beiden Ableitungen gezeigt werden kann:

$$
\frac{\partial \Pi^{Z B}}{\partial a}=\frac{1}{2} \cdot \frac{1}{\sqrt{\frac{2 \cdot(1-a)}{a \cdot(1+q)} \cdot \dot{\Pi}^{e}-\frac{1-q}{1+q} \cdot \frac{(1-a)^{2}}{a^{2}}}} .
$$

34 Im weiteren Verlauf wird aber davon ausgegangen, daß die Wirtschaftssubjekte einen Präferenzwandel zumindest in seiner Richtung erkennen. 


$$
\left[\frac{2}{1+q} \cdot \frac{-1 \cdot a-(1-a)}{a^{2}} \cdot \dot{\Pi}^{e}+\frac{2 \cdot(1-a)}{a \cdot(1+q)} \cdot \frac{\delta \dot{\Pi}^{e}}{\delta a}-\frac{1-q}{1+q} \cdot \frac{2 \cdot(1-a) \cdot(-1) \cdot a^{2}-(1-a)^{2} \cdot 2 \cdot a}{a^{4}}\right]
$$

$$
\frac{\partial \Pi^{\mathrm{ZB}}}{\partial \mathrm{a}}=\frac{1}{2 \cdot \sqrt{\frac{2 \cdot(1-\mathrm{a})}{\mathrm{a} \cdot(1+\mathrm{q})} \cdot \dot{\Pi}^{\mathrm{e}}-\frac{1-\mathrm{q}}{1+\mathrm{q}} \cdot \frac{(1-\mathrm{a})^{2}}{\mathrm{a}^{2}}}} \cdot\left[2 \cdot \frac{1-\mathrm{q}}{1+\mathrm{q}} \cdot \frac{1-\mathrm{a}}{\mathrm{a}^{3}} \cdot(1-\mathrm{x})\right]<0
$$

Da $x$ größer als Eins ist, wird die gesamte Lösung negativ, und somit sinkt $\Pi^{\mathrm{ZB}}$ mit steigendem a. Mit steigender Inflationsaversion sinkt die optimale Inflationsrate also, und die Reputation kann steigen. Entsprechendes gilt für q:

$$
\begin{aligned}
& \frac{\partial \Pi^{Z B}}{\partial q}=\frac{1}{2 \cdot \sqrt{\frac{2 \cdot(1-a)}{a \cdot(1+q)} \cdot \dot{\Pi}^{e}-\frac{1-q}{1+q} \cdot \frac{(1-a)^{2}}{a^{2}}}} \cdot \\
& {\left[\frac{2 \cdot(1-a)}{a \cdot(1+q)^{2}} \cdot(-1) \cdot \dot{\Pi}^{e}+\frac{2 \cdot(1-a)}{a \cdot(1+q)} \cdot \frac{\partial \dot{\Pi}^{e}}{\partial q}-\frac{(1-a)^{2}}{a^{2}} \cdot \frac{-(1+q)-(1-q)}{(1+q)^{2}}\right]} \\
& \text { 32) } \frac{\partial \Pi^{Z B}}{\partial q}=\frac{1}{2 \cdot \sqrt{\frac{2 \cdot(1-a)}{a \cdot(1+q)} \cdot \dot{\Pi}^{e}-\frac{1-q}{1+q} \cdot \frac{(1-a)^{2}}{a^{2}}}} \cdot\left[2 \cdot \frac{(1-a)^{2}}{a^{2} \cdot(1+q)^{2}} \cdot(1-x)\right]<0
\end{aligned}
$$

Auch dieser Ausdruck ist negativ, weil $\mathrm{x}$ größer als Eins ist. Als Ergebnis bleibt festzuhalten, daß die optimale Inflationsrate der Zentralbank um so geringer ist, je geringer die Inflationserwartungen und je höher ihre eigene Stabilitätsorientierung a sowie q, der Gewichtungsfaktor der Zukunft, sind. Je geringer aber die realisierte Rate wird, desto höher wird die Reputation.

Bevor im nächsten Kapitel diskutiert wird, wie die Bestimmungsgrößen der Reputation zu verändern bzw. beeinflussen sind, wird noch ein Vergleich mit dem Basismodell vorgenommen, welches ausschließlich eine glaubwürdige Geldpolitik unterstellt.

Bei glaubwürdiger Geldpolitik spielen die Inflationserwartungen keine Rolle für die optimale Inflationsrate der Zentralbank. Lediglich die Präferenzen der Zentralbank sowie deren Zeitpräferenzrate bestimmen diese Rate: $\Pi^{\mathrm{opt}}=\frac{1-\mathrm{q}}{1+\mathrm{q}} \cdot \frac{\mathrm{b}}{\mathrm{a}}$. Dies ändert sich jedoch, wenn die Wirtschaftssubjekte der Ankündigung der Zentralbank nicht glauben. Dann spielen die Erwartungen 
eine herausragende Rolle für die optimale Inflationsrate. Sobald keine Nullinflation erwartet wird, steigt die optimale Rate allein aufgrund der Inflationserwartungen. Thre Höhe bestimmt dann, bei gegebenen Präferenzen der Zentralbank, die optimale Inflationsrate. Es läßt sich zeigen, daß die optimale Inflationsrate bei unglaubwürdiger Antiinflationspolitik immer über der optimalen Inflationsrate bei glaubwürdiger Geldpolitik liegt:

$$
\frac{1-\mathrm{q}}{1+\mathrm{q}} \cdot \frac{\mathrm{b}}{\mathrm{a}} \leq \frac{\mathrm{b}}{\mathrm{a}} \cdot \sqrt{\frac{2 \cdot \mathrm{a}}{\mathrm{b} \cdot(1+\mathrm{q})} \cdot \dot{\Pi}^{\mathrm{e}}-\frac{1-\mathrm{q}}{1+\mathrm{q}}} \text { wenn gilt: } \dot{\Pi}^{\mathrm{e}} \geq \frac{1-\mathrm{q}}{1+\mathrm{q}} \cdot \frac{\mathrm{b}}{\mathrm{a}}
$$

Sobald die Wirtschaftssubjekte der Ankündigung der Zentralbank nicht glauben und ihre Erwartungen höher setzen, ist nur noch eine höhere Inflationsrate für die Zentralbank optimal. Der Aufbau von Reputation ist bei unglaubwürdiger Geldpolitik also nicht nur zum Preis einer Stabilisierungskrise zu erzielen, er wird auch einen längeren Zeitraum beanspruchen. Es wäre für die Reputation der Zentralbank also förderlich, wenn die Wirtschaftssubjekte die Geldpolitik als glaubwürdig ansehen würden. Dann wäre die „bessere“ optimale Inflationsrate des BG-Modells realisierbar. Allerdings muß nochmals die unterschiedliche Bedeutung der Erwartungen im BG-Modell und in der Erweiterung herausgestellt werden.

Bei BG handelt es sich nicht um Erwartungen im eigentlichen Sinne. Die Wirtschaftssubjekte kennen das Modell und haben alle Informationen, die die Politiker auch haben. Es liegt keine Unsicherheit vor. Der Erwartungsmechanismus war auch nicht konzipiert, um eventuelle Unsicherheiten zu senken, sondern ist als Drohmechanismus zu verstehen.

Die Wirtschaftssubjekte drohen eine adaptive Erwartungsbildung insofern an, als sie bei einer schlechten Politik in der nächsten Periode die schlechteste Inflationsrate erwarten würden und bei guter Politik auch für die nächste Periode mit einer solchen rechnen und demzufolge die optimale Inflationsrate erwarten. Allerdings stellt dieser Sanktionsmechanismus nur eine schwache Drohung dar, weil es sich um eine geringe Strafe handelt. Die Politiker wissen von vornherein, da $\beta$ sie eine Periode lang mit höheren Erwartungen zu rechnen haben und diese wieder senken können, wenn sie dann tatsächlich eine „schlechte“ Politik durchführen, was sie dann tun werden.

In der vorliegenden Modellerweiterung werden Inflationserwartungen gebildet, weil die Wirtschaftssubjekte unsicher sind bezüglich der Präferenzen der Zentralbank. Sie nutzen deshalb alle Informationen, die ihnen zugänglich sind. Mit jeder Handlung der Akteure nehmen diese Informationen zu und werden in den 
Inflationserwartungen berücksichtigt. Auch hier liegen rationale Erwartungen vor, da die Wirtschaftssubjekte die Anreizstrukturen der Zentralbank kennen. Asymmetrische Informationen aber führen dazu, daß sie über die Präferenzen der Zentralbank nur Vermutungen anstellen können, die sie aus allen ihnen zugänglichen Informationen bilden.

Für die Reputation bedeutet dies, daß es sich im Grundmodell um eine objektive Größe handelt, die richtig oder falsch sein kann, da die Öffentlichkeit die Politikerpräferenzen kennt. Im vorliegenden Modell wird die Reputation als Meinung der Öffentlichkeit über die Akteure verstanden. $\mathrm{Ob}$ diese Meinung richtig oder falsch ist, spielt hierbei keine Rolle. Die Zentralbank kann diese Meinung aber durch eine entsprechende Politik im positiven oder negativen Sinne beeinflussen.

Die Folge einer positiven Reputation ist in beiden Modellen die gleiche, sie kann die Inflationsrate senken, ohne zusätzlich Kosten in Form steigender Arbeitslosigkeit hinnehmen zu müssen, was zugleich die Bedeutung der Reputation für eine erfolgreiche Antiinflationspolitik zum Ausdruck bringt. Es müssen deshalb die die Reputation bestimmenden Größen näher untersucht werden. Im Basismodell hat lediglich die zuletzt realisierte Inflationsrate die Reputation bestimmt. In der Erweiterung wurde sie als von mehreren Variablen abhängig betrachtet: dem Stabilitätsbewußtsein a bzw. der Vorliebe zur Beschäftigungsstimulierung $b$, der Zukunftsorientierung $q$ und den Inflationserwartungen $\dot{\Pi}^{\mathrm{e}}$, die zugleich über die Glaubwürdigkeit der Geldpolitik entscheiden. Diese Größen wiederum sind alle von $\gamma$, dem Machtparameter der Zentralbank, abhängig.

Das beschriebene Modell war auch notwendig, um die die Reputation bestimmenden („direkten“) Determinanten herauszuarbeiten. Für die weitere Arbeit besteht das Ziel nun darin, zu untersuchen, welche Größen wiederum die „direkten“ Determinanten bestimmen, bezüglich der Reputation also „indirekte“ Determinanten darstellen. Anhand dieser Analyse kann also gezeigt werden, wie eine Zentralbank eine möglichst hohe Reputation erlangen könnte. Dies ist die Fragestellung für das folgende Kapitel, in dem der modelltheoretische Rahmen um den Analyserahmen der Neuen Politischen Ökonomie ergänzt wird. 
Iris Henning - 978-3-631-75125-1

Downloaded from PubFactory at 01/11/2019 08:02:27AM

via free access 
Im Gegensatz zum BG-Modell, das die Reputation als ausschließlich von der Leistung abhängig sieht, wird sie hier von mehreren Determinanten bestimmt. Die Leistung - im Sinne der Realisierung geringer Inflationsraten - kann anhand realisierter Ergebnisse und bestehender Anreize zur Inflations- bzw. Antiinflationspolitik beurteilt werden. Letztere sind insbesondere dann wichtig, wenn eine Zentralbank erst neu gegründet wird und demzufolge nicht auf Erfahrungswerte zurückgegriffen werden kann. Bei der Analyse der Antiinflationspolitik ist es also sinnvoll, zunächst die Inflationsanreize zu untersuchen, um potentiell Begünstigte der Inflation zu ermitteln. Dies geschieht in Kapitel 3.1 mit dem Ergebnis, daß insbesondere die Regierung, d.h. wahlabhängige Politiker, Anreize für eine Inflationspolitik haben.

Im Kapitel 3.2 wird weiterhin gezeigt, daß die für die Reputation notwendige Glaubwürdigkeit der Geldpolitik wiederum für Politiker ein besonderes Problem darstellt. Wenn die Geldpolitik von Politikern bestimmt wird, ist sie auf drei Ebenen nicht glaubwürdig: Eine Antiinflationspolitik ist zum ersten aus politischen Gründen nicht voll glaubwürdig, weil demokratisch gewählte Regierungen aufgrund der Wahlzyklen den Inflationsanreizen nachgeben werden. Zweitens kann aufgrund strategischer Überlegungen die optimale Inflationsrate so lange nicht erreicht werden, wie die Politiker noch einen diskretionären Spielraum in der Geldpolitik besitzen. Für die Zentralbank werden die für die Politiker geltenden Anreize und Probleme dann relevant, wenn die Zentralbank nur ausführendes Organ der Regierung ist. In diesem Fall sind die Ziele der Zentralbank mit denen der Regierung identisch, und es ist letztlich irrelevant, ob die Geldpolitik durch von der Regierung abhängige Zentralbanker oder direkt von den Politikern durchgeführt wird. Drittens kommt es zusätzlich, ob die Zentralbank abhängig ist oder nicht, zu einem „technologischen“ Glaubwürdigkeitsproblem, wenn die Wirtschaftssubjekte davon überzeugt sind, daß die Geldpolitik wegen von der Zentralbank nicht zu kontrollierender Störfaktoren im Transmissionsprozeß nicht entsprechend ihrer Zielsetzung wirken kann. ${ }^{1}$

Insgesamt zeigt sich, daß eine notwendige Bedingung für eine auf allen Ebenen glaubwürdige Antiinflationspolitik darin besteht, daß die Regierung ihre Macht über die Geldpolitik an eine unabhängige Zentralbank abtritt. Deshalb wird im Kapitel 3.3 zunächst die juristische Seite der Unabhängigkeit diskutiert. Da es aber nicht gesichert ist, daß diese formelle Macht auch faktisch umgesetzt

$1 \quad$ Zu dieser Einteilung der Glaubwürdigkeitsprobleme vgl. Hamacher (1995). 
wird, muß die tatsächliche Macht hinterfragt werden. Man kann hier auch von informeller Macht der Zentralbank sprechen.

Die volle Unabhängigkeit der Zentralbank in formeller und informeller Hinsicht stellt aber noch keine hinreichende Bedingung für eine Antiinflationspolitik dar. Wenn im Sinne der Neuen Institutionenökonomie von einer eigennutzmaximierenden Zentralbank ausgegangen wird, stellt sich die Frage, inwieweit eine Antiinflationspolitik der Interessenlage der Zentralbanker entspricht. Bestehen hier potentielle Diskrepanzen, ist zu untersuchen, ob Kontrollmechanismen existieren, die die Zentralbank zum gewünschten Verhalten „Zwingen“. Sollten solche Mechanismen nicht existieren, müssen den Zentralbankern Anreize gesetzt werden, die es aus ihrer Sicht optimal erscheinen lassen, eine glaubwürdige Antiinflationspolitik zu verfolgen und somit die gesellschaftliche Wohlfahrt zu maximieren. Als mögliche Anreize werden im Kapitel 3.4 die Öffentlichkeitsarbeit, eine Regelbindung sowie persönliche Anreize für die Zentralbanker analysiert.

Bei der gesamten Diskussion ist zu beachten, daß die beschriebenen Faktoren nicht nur auf die die Reputation bestimmenden Determinanten (a, b, q, $\dot{\Pi}^{\mathrm{e}}$ ) wirken, sondern umgekehrt die Reputation auch die beschriebenen Faktoren beeinflußt. Es handelt sich hier also um ein Rückkopplungsmodell.

\subsection{Die Leistung einer Zentralbank}

\subsubsection{Erfahrungen als Indiz für die Leistung}

Wie aus Gleichung (2.17) hervorgeht, bestimmt die Zentralbank die Inflationsrate nicht allein. Auch die Fiskal- und Tarifpolitik sowie außenwirtschaftlich bedingte Störungen beeinflussen sie zumindest kurzfristig. Die Inflationsrate, die von den Wirtschaftssubjekten beobachtet werden kann, stellt deshalb keinen sicheren Indikator für die Leistung der Zentralbank dar. Sie kann den Wirtschaftssubjekten immer nur Anhaltspunkte über das Verhalten der Zentralbank liefern.

Die Bewertung der kurzfristigen Inflationsrate hängt aber auch von der Öffentlichkeitsarbeit der Zentralbank ab. Sie kann durch öffentliche Erklärungen dazu beitragen, daß die Wirtschaftssubjekte besser einschätzen können, welchen Teil der Inflationsrate die Fiskal- bzw. die Tarifpolitiker „verschuldet" haben. Hier wird die gegenseitige Abhängigkeit von Leistung und Reputation besonders deutlich. Genießt die Zentralbank schon eine hohe Reputation, werden die Wirtschaftssubjekte ihr eher glauben, als wenn sie noch keine bzw. erst eine sehr geringe Reputation aufgebaut hat. Im Falle einer hohen Reputation ist es 
sogar denkbar, daß es der Zentralbank gelingt, einen Teil der von ihr „verschuldeten" Inflationsrate auf die Regierung bzw. Tarifpartner abzuschieben. Allerdings hat sie diese Möglichkeit nicht über unendlich viele Perioden, da es irgendwann unglaubwürdig wird und sie an Reputation verliert.

Hat die Zentralbank im umgekehrten Fall noch keine oder nur eine geringe Reputation, ist es möglich, daß sie für die von anderen zu verantwortenden Teile der Inflationsrate verantwortlich gemacht wird und ihr damit die Möglichkeit zum Reputationsaufbau zumindest erschwert wird.

Da die kurzfristige Inflationsrate nur ein unsicherer Indikator für das Verhalten der Zentralbank ist, ist es sinnvoll, einen zusätzlichen Indikator heranzuziehen, der genauere Aussagen über das tatsächliche Verhalten der Zentralbank liefern kann. Eine solche Größe stellt die langfristige Inflationsrate dar, weil langfristig jede Inflation von der Zentralbank finanziert werden muß.

Problematisch kann die Beurteilung der Leistung jedoch werden, wenn die Zentralbank noch nicht (lange) existiert und noch keine Erfahrungswerte vorliegen, aus denen die Wirtschaftssubjekte ihre Informationen ziehen können. Dann bleibt ihnen ausschließlich die Möglichkeit, die sehr kurzfristig realisierten Inflationsraten zu Hilfe zu nehmen, die die beschriebenen Unsicherheiten beinhalten. Wird eine Zentralbank sogar erst neu gegründet und empirische Daten liegen noch nicht vor, können nur Vermutungen über das Verhalten der Zentralbank angestellt werden. Ein Indiz für das zu erwartende Verhalten stellen die Anreize dar, denen die Akteure unterliegen. Als erste Frage stellt sich deshalb, ob es im Sinne der Zentralbank ist, eine Inflation zu vermeiden bzw. geringe Raten anzustreben, oder ob sie aus der Inflation Vorteile ziehen kann.

\subsubsection{Inflationsanreize}

Ob eine Zentralbank Vorteile aus einer Inflation zieht oder nicht, wird wesentlich von ihrem Abhängigkeitsgrad von der Regierung bestimmt. Wird die Geldpolitik de facto von der Regierung bestimmt, unterliegt die Zentralbank starken Inflationsanreizen, da die Inflation nicht nur direkte Vorteile für die Regierung ermöglicht, sondern auch zur Verbesserung der Position bestimmter Wählergruppen beitragen kann. Eine demokratisch legitimierte Regierung ist immer wahlabhängig und zieht deshalb auch Vorteile daraus, wenn sie bestimmte Gruppen begünstigt, da so ihre Wiederwahlwahrscheinlichkeit zunehmen kann.

Die direkten Vorteile für die Regierung aus einer Inflation ergeben sich aus einer besseren Finanzierungsmöglichkeit des staatlichen Budgets. In erster Li- 
nie ist hier an Seigniorage-Gewinne der Regierung zu denken. ${ }^{2}$ Sie resultieren aus der Einnahmesteigerung aus der Emission von Zentralbankgeld. Insofern stellt die Inflation eine Art Steuer auf die Kassenhaltung dar. ${ }^{3}$ Obwohl bei zunehmender Inflationsrate die Wirtschaftssubjekte ihre Realkassenhaltung mindern, steigt die nominelle Geldmenge, d.h. die Wirtschaftssubjekte müssen sich den Nutzen der Geldhaltung bei Inflation mit dem Geldemittenten teilen. ${ }^{4}$ Dieser Nutzen des Geldproduzenten wird als Inflationssteuer interpretiert. Ein Mangel an anderen effizienten Steuerquellen könnte einen Anreiz für die Regierung zur Inflationierung darstellen. Es wurden bereits Modelle zur Frage entwickelt, welche „Inflationspolitik“ zum maximalen Steueraufkommen führt.

Neben diesen Seigniorage-Gewinnen zieht die Regierung aber noch weitere Vorteile aus den Umverteilungseffekten der Inflation. ${ }^{5}$ Nach der Transfereinkommens-Hypothese erhalten Bezieher von Nicht-Markteinkommen, z.B. Rentner und Sozialhilfeempfänger, keinen vollen Inflationsausgleich. Da eine Erhöhung dieser Einkommen immer eine politische Entscheidung ist bzw. einer gesetzlichen Regelung bedarf, wird die Entwicklung zumindest mit einer Zeitverzögerung verbunden sein.

Entsprechend der Nettoeinkommens-Hypothese steigen in einer inflationären Volkswirtschaft die realen Steuererträge, ohne daß die realen Einkommen steigen. Bei Vorliegen einer progressiven Einkommenssteuer nehmen die Steuereinnahmen des Staates überproportional zum Einkommen zu, was auch als „kalte Progression“ bezeichnet wird. 6

Aber nicht nur die Einkommensseite des Staates wird von einer Inflationierung positiv betroffen, auch die Ausgabenseite wird begünstigt. So geht nicht nur die reale Zinsbelastung zurück, da dem Realzinsargument folgend die nominelle Zinsentwicklung der Preisniveausteigerung nicht entspricht, auch die gesamte Staatsschuld wird durch ihren sinkenden Realwert verringert. Letzteres ist die

2 Eine modelltheoretische Analyse von Glaubwürdigkeitsaspekten der Geldpolitik unter Berücksichtigung der Fiskalpolitik, insbesondere der Seigniorage-Gewinne, nehmen Alesina/Tabellini (1987) vor.

3 Die Literatur zur Inflation als Steuer hat heute ein beträchtliches Ausmaß erreicht. Eine Literaturübersicht geben z.B. Tower (1971) und Tatom (1976).

4 Der Punkt des Wohlfahrtsverlustes durch eine suboptimale Kassenhaltung bei Inflation wurde zum ersten Mal von Bailey (1956) erwähnt.

5 Vgl. hierzu z.B. Fricke (1981).

6 Zusätzliche Steuern erhält der Staat in Zeiten der Inflation auch durch die Besteuerung von Scheingewinnen. Diese fallen an, weil die Unternehmen ihre Gewinne aufgrund des Nominalwertprinzips berechnen müssen. Tatsächlich sind die vergangenen Werte mit den heutigen aber nicht mehr vergleichbar durch die Inflation. 
Aussage der Gläubiger-Schuldner-Hypothese, nach der das Finanzvermögen zugunsten der Schuldner verlagert wird. Da traditionell der Staat und der Unternehmenssektor die Schuldnerposition in einer Volkswirtschaft einnehmen, werden diese beiden Gruppen in dieser Hinsicht durch eine Inflationierung begünstigt. Zusätzlich wird das Realvermögen von den Gläubigern zu den Schuldnern umverteilt, da die Inflation Forderungstitel bei Nominalwertfixierung entwertet im Gegensatz zum Realvermögen. Wieder ist der Unternehmenssektor hiervon begünstigt.

Schließlich bedingt eine Inflation auch eine Begünstigung der Gewinnquote zu Lasten der Lohnquote, da entsprechend der Lohn-lag-Hypothese die Lohnerhöhungen hinter den Preissteigerungen zurückbleiben.

Die letzten drei Hypothesen werden unter dem „Finanzierungsargument“ zusammengefaßt, da die Umverteilung zugunsten des Unternehmenssektors zugleich eine Begünstigung der Investitionsfinanzierung darstellt, wovon letztlich auch die Arbeitnehmer positiv betroffen sein können, wenn es durch zunehmende Investitionen zu Beschäftigungseffekten kommt.

Ein weiterer Inflationsanreiz resultiert aus der kurzfristigen Phillips-Kurve. Danach können kurzfristige Beschäftigungseffekte direkt durch eine Inflationierung herbeigeführt werden, sofern sie für die Wirtschaftssubjekte überraschend kommt. ${ }^{7}$ Institutionelle Hemmnisse, wie sie in der Realität durch längerfristige Arbeitsverträge gegeben sind, führen zu zeitweisen Lohnstarrheiten und verhindern eine spontane Anpassung an die veränderten Marktdaten. Diese langfristigen Bindungen wirken faktisch wie das Vorliegen von Geldillusion. ${ }^{8}$ Andererseits können die erforderlichen Anpassungen auch ausbleiben, weil sie nicht kostenlos realisierbar sind. Es können erhebliche Transaktions- und Informationskosten anfallen.

Auch wenn diese Beschäftigungseffekte bestenfalls nur kurzfristig wirksam sind, stellen sie für kurzfristig denkende Politiker erhebliche Inflationsanreize dar. Dies gilt insbesondere kurz vor Wahlen, da sie so ihre Wiederwahlwahrscheinlichkeit positiv beeinflussen können.

Aufgrund der genannten kurzfristigen Vorteile in Form der Beschäftigungszunahme und Umverteilung zugunsten der Regierung ist es fragwürdig, ob kurzfristig denkende wahlabhängige Politiker eine dauerhafte Stabilitätspolitik ver-

7 Vgl. hierzu auch die Ausführungen in Kapitel 2.1.

8 Dieser Ansatz, der trotz rationaler Erwartungsbildung zu kurzfristigen Beschäftigungseffekten führt, stammt von Fischer (1977). 
folgen werden. Spätestens vor Wahlterminen unterliegen sie einem starken Anreiz zur Inflation, da so nicht nur kurzfristig die Beschäftigung gesteigert und Wahlgeschenke ,günstig“ finanziert werden können, sondern auch bestimmte Wirtschaftsgruppen aufgrund der für sie günstigen Umverteilungseffekte ein Interesse an der Inflation haben könnten.

Wenn die Wirtschaftssubjekte ihre Erwartungen rational bilden, kennen auch sie diese Anreize und werden eine abhängige Zentralbank kaum mit Reputation „belohnen“. Zumindest wird es einer abhängigen Zentralbank schwerer fallen, Reputation aufzubauen, als einer Zentralbank, die ihre Entscheidungen unabhängig vom tagespolitischen Geschehen treffen kann. Um einen wirksamen Schutz vor der Inflation zu gewährleisten, muß demnach eine langfristig denkende, von der Regierung unabhängige Instanz, die von Fachleuten geführt wird, die Geldpolitik einer Volkswirtschaft bestimmen.

\subsubsection{Die Bedeutung der Leistung für das Modell}

Es wurde modelltheoretisch gezeigt, daß die Reputation vom Stabilitätsbewußtsein und der Zeitpräferenzrate der Zentralbank sowie der Einschätzung der Wirtschaftssubjekte bezüglich dieser beiden Größen bestimmt wird. Aufgrund der von der Zentralbank erbrachten Leistung schließen die Wirtschaftssubjekte auf deren Stabilitätsbewußtsein und Gegenwartsvorliebe. Dabei wissen die Wirtschaftssubjekte, daß die Inflationsrate, die sie beobachten, nicht ausschließlich die Leistung der Zentralbank widerspiegelt, da sich die tatsächlich realisierte Inflationsrate entsprechend Gleichung (2.17) aus einem von der Zentralbank zu verantwortenden und einem von ihr nicht $\mathrm{zu}$ kontrollierenden Teil zusammensetzt. Es wird zwar angenommen, daß dieser letzte Teil in der Regel etwa $2 \%$ beträgt, dennoch wissen auch die Wirtschaftssubjekte, daß es vereinzelt $\mathrm{zu}$ anderen Werten kommen kann.

Für den Fall einer völlig gebundenen Zentralbank $(\gamma=0)$ müssen die Inflationsanreize für die Regierung hinterfragt werden. Wie sich bei der diesbezüglichen Diskussion gezeigt hat, ziehen Politiker mehrere Vorteile aus einer positiven Inflationsrate, egal ob diese antizipiert ist oder nicht. Besonders wichtig sind die kurzfristigen Beschäftigungseffekte. Da die Regierung von den Wirtschaftssubjekten nicht nur für ein einziges Ziel verantwortlich gemacht wird, kann es nicht dazu kommen, daß Politiker ausschließlich a gewichten und b unberücksichtigt lassen.

Wie gezeigt wurde, ist die jeweilige Gewichtung auch von der Zielrealisation abhängig. Als Problemfall hat sich die Situation erwiesen, in der beide Ziele verfehlt sind. Hier werden Politiker beiden Zielen einen positiven Nutzen bei- 
messen. Die Inflationsaversion von Politikern wird also nicht vollkommen sein, solange das Beschäftigungsziel noch nicht befriedigend realisiert ist (es gilt $a=1-b$, deshalb ist $a<1$, solange noch $b>0$ gilt). Da auch die Wirtschaftssubjekte dies wissen, werden sie der Zentralbank nicht die höchste Reputation zugestehen, solange noch Politiker Einfluß auf die Geldpolitik haben. Modelltheoretisch bedeutet dies, daß die Reputation nur suboptimale Werte annehmen wird, solange der Machtparameter der Zentralbank noch seinen Maximalwert unterschreitet, d.h. $\gamma<1$ gilt.

Gleiches gilt auch für den Diskontierungsfaktor q. Weder werden Politiker die Zukunft genauso stark gewichten wie die Gegenwart, noch werden die Wirtschaftssubjekte daran glauben, daß Politiker dies tun. Politiker denken eher kurzfristig, da ihr Zeithorizont in der Regel nur die Länge einer Legislaturperiode beträgt. Eine positive Wirkung auf die Reputation hätte es demnach, wenn nicht Politikerpräferenzen wichtig wären, sondern die einer unabhängigen Instanz, die langfristig orientiert handeln könnte und aus der Inflation keine Vorteile zieht. Eine unabhängige Zentralbank $(\gamma \rightarrow 1)$ würde die tatsächliche Inflationsrate auch in zunehmendem Maße beeinflussen, da sie im Idealfall die komplette Macht über die Geldpolitik hätte (vgl. Gleichung (2.18)) und andererseits, bei entsprechender Definition der Unabhängigkeit, auch die Einflußnahme der Regierung auf die Inflationsrate über die Fiskalpolitik zurückgehen könnte. Entsprechend Gleichung (2.17) könnte die tatsächlich realisierte Inflationsrate dann zurückgehen.

Wie anhand des Modells gezeigt wurde, stellen die Inflationserwartungen der Wirtschaftssubjekte eine wichtige Determinante für die optimale Inflationsrate der Zentralbank dar. Sobald die Wirtschaftssubjekte der Ankündigung der Zentralbank nicht glauben, muß letztere die Inflationserwartungen in ihrem Kalkül berücksichtigen. Auch wenn die Zentralbank bei der Berechnung ihrer Ankündigung und ihrer tatsächlichen Realisation von gleichen Präferenzen ausgeht, können beide Werte erheblich voneinander abweichen, je nach Höhe der Erwartungen, was das folgende Beispiel verdeutlicht:

Die Zentralbank kündigt zu Beginn der Periode aufgrund ihrer Präferenzen eine Inflationsrate von $0,2 \%$ an, welche sich aus den folgenden Werten ergibt:

$$
\begin{aligned}
& a=0,98 \quad b=0,02 \quad q=0,8 \\
& \Pi^{a}=\frac{b}{a} \cdot \frac{1-q}{1+q}=0,0022=0,2 \%
\end{aligned}
$$


Unterstellen die Wirtschaftssubjekte der Zentralbank aber nur eine Stabilitätsaversion von $a=0,8$, ergibt sich für die Inflationserwartung der folgende Wert:

$$
\Pi^{\mathrm{e}}=\frac{\mathrm{b}}{\mathrm{a}} \cdot \frac{1-\mathrm{q}}{1+\mathrm{q}}=0,027=2,7 \%
$$

Da die Geldpolitik aufgrund der Inflationserwartungen unglaubwürdig für die Wirtschaftssubjekte ist, muß die Zentralbank bei der Berechnung ihrer optimalen Inflationsrate nun auch die Inflationserwartungen berücksichtigen. Es ergibt sich somit der folgende Wert:

$$
\Pi \quad \frac{\mathrm{b}}{\mathrm{a}} \sqrt{\frac{2 \mathrm{a}}{\mathrm{b}(1 \mathrm{q})} \Pi \frac{1 \mathrm{q}}{1 \mathrm{q}}} \quad 0,023 \quad 2,3 \%
$$

Setzt man nun für die Inflationserwartungen andere Werte ein, ergeben sich auch andere optimale Inflationsraten:

$$
\begin{aligned}
& \Pi^{\mathrm{e}}=4 \% \quad \Rightarrow \quad \Pi^{\mathrm{ZB}}=2,9 \% \\
& \Pi^{\mathrm{e}}=1 \% \quad \Rightarrow \quad \Pi^{\mathrm{ZB}}=1,3 \%
\end{aligned}
$$

Selbst wenn die sehr stabilitätsbewußte Zentralbank $(\mathrm{a}=0,98)$ zu Beginn der Periode eine Inflationsrate von 0,2 realisieren wollte und diese auch ankündigt, wird sie diesen Wert nicht realisieren können, solange die Wirtschaftssubjekte nicht an die Ankündigung glauben. Je höher die Erwartungen der Wirtschaftssubjekte sind, desto höher fällt auch die optimale Inflationsrate der Zentralbank aus, da sie bei der Berechnung nun auch die Stabilisierungskrise berücksichtigen muß, die sie durch ihre Antiinflationspolitik verursacht $\left(\Pi^{\mathrm{ZB}}<\Pi^{\mathrm{e}}\right)$. Es gilt:

Nur bei einer glaubwürdigen Geldpolitik könnte eine Stabilisierungskrise ausbleiben. Wie ausgeführt wurde, bilden die Wirtschaftssubjekte ihre Inflationserwartungen aufgrund vergangener realisierter Werte und der für die Zentralbank gültigen Rahmenbedingungen. Ist eine Zentralbank von Politikern abhängig, wissen auch die Wirtschaftssubjekte, daß trotz eines ausgeprägten Stabilitätsbewußtseins der Zentralbank nicht deren Präferenzen über die Geldpolitik entscheiden, sondern die Präferenzen der Politiker. Letztere ziehen aus einer Inflationierung aber tendenziell Vorteile. Selbst wenn die Politiker den Inflationsanreizen nicht nachgeben wollen, ist es zumindest schwierig, dies den Wirtschaftssubjekten zu verdeutlichen. Eine unabhängige Zentralbank könnte des- 
halb zur Senkung der Inflationserwartungen beitragen, wie es auch durch Formel (2.19) zum Ausdruck kommt:

$$
\Pi^{\mathrm{e}}=\gamma \cdot \Pi_{\mathrm{ZB}}^{\mathrm{e}}+(1-\gamma) \cdot \Pi_{\mathrm{P}}^{\mathrm{e}}+2 \%
$$

Da die Wirtschaftssubjekte davon ausgehen, daß $\Pi_{\mathrm{ZB}}^{\mathrm{e}}$ aufgrund der Inflationsanreize immer geringer ist als $\Pi_{\mathrm{P}}^{\mathrm{e}}$, sind die Inflationserwartungen um so geringer, je höher $\gamma$, also größer die Unabhängigkeit der Zentralbank ist.

Es bleibt festzuhalten, daß allein durch Abgabe der Geldpolitik von den Politikern an eine unabhängige Zentralbank die Reputation steigen kann, was sich in sinkenden Inflationserwartungen widerspiegelt und letztlich zu geringeren optimalen Inflationsraten führt. Dies Ergebnis gilt bereits aufgrund der Tatsache, daß Inflationsanreize für Politiker nur existieren. Ob diese Anreize auch ausgenutzt werden, ist zunächst noch unwichtig. Allein die Möglichkeit zur Abweichung von einer stabilitätsorientierten Geldpolitik läßt die Wirtschaftssubjekte vorsichtiger disponieren.

\subsection{Die Glaubwürdigkeit der Geldpolitik}

\subsubsection{Das ,politische“ Glaubwürdigkeitsproblem}

Nun soll gezeigt werden, daß die Regierung als potentieller Nutznießer der Inflation diesen Nutzen auch wird realisieren wollen. Die Ursache dafür liegt im politischen Wahlsystem, d.h. eine strikte Antiinflationspolitik kann am ,politischen" Glaubwürdigkeitsproblem scheitern. Dieser Punkt der politischen Ursachen der Inflation wird zwar oft vernachlässigt, muß aber berücksichtigt werden, da die Geldpolitik nicht im ,politikfreien“ Raum stattfindet.

Einen möglichen Erklärungsansatz für eine ,politisch“ bedingte Inflation bietet die Theorie des politischen Konjunkturzyklus. ${ }^{9}$ Politiker werden als Eigennutzmaximierer betrachtet und sind deshalb ausschließlich an ihrer Wiederwahl interessiert. Entscheidend für die Wiederwahl ist die Popularität der Regierung, welche wiederum signifikant von der Inflationsrate und der Arbeitslosenquote beeinflußt wird. ${ }^{10} \mathrm{Da}$ die Wirtschaftssubjekte ihre Erwartungen adaptiv, also rückwärtsschauend bilden, können die Politiker kurzfristig den PhillipsKurven-Trade-off nutzen und kurz vor der Wahl die Arbeitslosigkeit senken, was nach der Wahl mit einer steigenden Inflationsrate „bezahlt“ werden muß.

9 Der bekannteste Ansatz ist der von Nordhaus (1975).

10 Zur Erklärung der Popularitätsfunktion und für weiterführende Literatur vgl. Dittus (1987), S. 155f. 
Die Wirtschaftssubjekte werden bei ihrer Entscheidung aber nur die günstige Situation zum Wahlzeitpunkt berücksichtigen, weil sie annahmegemäß kurzfristig denken.

Es kann gezeigt werden, daß die wesentlichen Ergebnisse dieses Ansatzes auch bei Existenz rationaler Erwartungen Bestand haben, sobald Informationsasymmetrien vorliegen. ${ }^{11}$ Entsprechend der Theorie des politischen Konjunkturzyklus orientieren sich die Politiker immer am Medianwähler, und deshalb werden sich die wirtschaftspolitischen Programme der unterschiedlichen Parteien stark angleichen. Da diese Modelle aber eher die Situation in den USA als in Europa erklären können, sollen sie hier nicht weiterverfolgt werden. Für Europa ist eine alternative Verhaltensannahme geeigneter, das politische Handeln zu erklären. Entsprechend der „Partisanentheorie“ bzw. ihrer Weiterentwicklung unter Berücksichtigung rationaler Erwartungen als sogenannte „Rationale Partisanentheorie" handeln die Parteien entsprechend einer Ideologie, die sich aus den Präferenzen der Stammwählerschaft ableiten läßt. ${ }^{12}$

In der Regel stehen sich eine „linke“ oder „progressive“ und eine „rechte“ bzw. „konservative“ Partei gegenüber. Die Stammwählerschaft der „linken“ Partei ist der unteren Mittelklasse zuzurechnen, die eher von einer Kombination niedriger Arbeitslosigkeit bei höherer Inflation profitiert. Die „rechte“ Partei hingegen wird von der oberen Mittelklasse gestützt, die eine geringere Inflation höher gewichtet als die Arbeitslosigkeit. Dementsprechend präferieren ,linke“ Parteien eine expansive Wirtschaftspolitik bei geringerer Abneigung gegen eine Inflation, während „rechte“ Parteien eher die Inflation bekämpfen und die Staatsausgaben mindern wollen.

Die Volkswirtschaft wird durch eine kurzfristige Phillips-Kurve beschrieben. $\mathrm{Zu}$ nominalen Rigiditäten kommt es aufgrund der Existenz langfristig gültiger nicht-indexierter Arbeits- bzw. Lohnverträge. Es finden regelmäßig demokratische Wahlen statt. Diese Wahlen bergen für die Volkswirtschaft Risiken, da die Wirtschaftssubjekte vorher nicht wissen, welche Partei die Regierung stellen wird. Die Ungewißheit folgt aus der Tatsache, daß nur ein Teil der Wählerschaft den Stammwählern zuzurechnen ist und die Wechselwähler somit über den Wahlausgang entscheiden. Die Wirtschaftssubjekte müssen demnach ihre

11 So berücksichtigen Cukierman/Meltzer ökonomische Schocks, die die Wirtschaftssubjekte nicht erkennen können, und Rogoff argumentiert mit Informationsasymmetrien hinsichtlich der Fähigkeiten der Politiker. Vgl. Cukierman/Meltzer (1986c) und Rogoff (1987b) sowie Rogoff/Siebert (1988).

12 Zum ursprünglichen Ansatz vgl. Hibbs (1977). Zur Erweiterung, die hier als Grundlage dient, vgl. Alesina (1987), (1988), (1989) und Alesina/Sachs (1988). 
Nominallohnverträge vor der Wahl unter Unsicherheit abschließen. Zwar kennen sie die konsistenten Inflationsraten der beiden Parteien, da sie aber nicht wissen, welche Partei die Wahl gewinnen wird, werden sie eine gewichtete Inflationsrate aus beiden erwarten. Die Wirtschaftssubjekte wissen, daß eine „rechte“ Regierung (im Extremfall) nach der Wahl eine Nullinflation realisieren wird:

(3.1) $\Pi_{R}=0$

Inflationsrate einer ,rechten“ Regierung

Eine „linke“ Regierung bevorzugt jedoch das Beschäftigungsziel und kann dementsprechend nur die schlechtere diskretionäre Inflationsrate realisieren:

(3.2) $\quad \Pi_{L}=\frac{b}{a}$

Inflationsrate einer „linken“ Regierung

Da die Wirtschaftssubjekte nicht wissen, welche Partei an die Regierung kommt, werden sie eine mittlere Inflationsrate erwarten, die mit der jeweiligen Wahrscheinlichkeit für einen Wahlsieg gewichtet wird und sich aus der Verteilung der Stammwähler ergibt. Bei der Wahl ihrer Inflationserwartungen können die Wirtschaftssubjekte somit nicht die Wechselwähler berücksichtigen:

$$
\Pi^{\mathrm{e}}=\mathrm{p} \cdot \Pi_{\mathrm{R}}+(1-\mathrm{p}) \cdot \Pi_{\mathrm{L}} \quad \text { mit: } \Pi_{\mathrm{R}}<\Pi_{\mathrm{L}}
$$

Kommt es nun zu einem Wahlsieg der „rechten“ Partei, was mit einer Wahrscheinlichkeit von $p$ der Fall ist, wird sie die Wirtschaft temporär in eine Rezession führen, da sie eine Inflationsrate realisiert, die unterhalb der erwarteten liegt. Bei einem Wahlsieg der "linken“ Partei erlebt die Volkswirtschaft zunächst eine Boomphase durch die Überraschungsinflation. Die „linke“ Partei realisiert eine höhere Inflationsrate, als die Wirtschaftssubjekte dies erwartet haben, weil in die Erwartungen auch die gewichtete Nullinflation der rechten Partei eingeht. Sobald die Wirtschaftssubjekte ihre Erwartungen aber aufgrund neuer Lohnverhandlungen richtig antizipieren können, werden sie nicht mehr getäuscht, da sie die konsistenten Inflationsraten der Parteien kennen. Die höhere Inflationsrate der ,linken“ Partei kommt dann nicht mehr überraschend, und die positiven Beschäftigungseffekte bleiben aus. Es stellt sich ein Outputniveau ein, welches sich auch unter einer ,rechten“ Regierung ergibt. Allerdings bleibt die Inflation unter der ,linken“ Regierung signifikant höher.

Aus der bisherigen Erörterung scheint es, daß nur unter einer „linken“ Regierung positive Inflationsraten auftreten. Solange „rechte“ Parteien regieren, stellt die Inflation kein Problem dar, und das einzige Problem ist in der Rezession zu Beginn der Amtszeit zu sehen. Diese Aussage ist aber weder theore- 
tisch noch empirisch haltbar. Theoretisch läßt sich dies relativieren, wenn man beide Theorien, die des politischen Konjunkturzyklus und die „Rationale Partisanentheorie“, miteinander verbindet. ${ }^{13}$

Es ist durchaus realistisch, daß eine Partei ihrem ideologischen Konzept folgt, solange ihre Wiederwahl gesichert erscheint. Sobald dies nicht mehr gegeben ist, wird sie sich an den Präferenzen der Wechselwähler orientieren. In dieser Situation unterscheiden sich die Ziele einer ,linken“ und „rechten“ Partei nicht mehr, beide verfolgen dann die Maximierung des Stimmanteils. Da die Wechselwähler die Regierung vor allem über ihre aktuelle Leistung auf dem Gebiet der Wirtschaftspolitik beurteilen, besteht ein Anreiz zur Herbeiführung eines „Vorwahlbooms". Über eine expansive Wirtschaftspolitik wird die Regierung versuchen, die Beschäftigung und damit das Wirtschaftswachstum günstig zu beeinflussen. Ein weiterer Anreiz zur Überraschungsinflation besteht in dem zusätzlich gewonnenen Ausgabenspielraum durch die reale Entwertung der Staatsschuld bzw. der Inflationssteuereinnahmen, den sie für Wahlgeschenke an die Wechselwähler nutzen kann. Diese werden durch die direkte Begünstigung der Regierung tendenziell positiv gegenüberstehen. ${ }^{14}$ Es kann gezeigt werden, da $\beta$ dieser Anreiz zur Inflationierung für Koalitionsregierungen noch zunimmt. 15

Neben den Glaubwürdigkeitsproblemen, die in direkter Verbindung mit der Wahl selbst stehen, können Politiker aber auch innerhalb einer Legislaturperiode Probleme haben, Glaubwürdigkeit für eine Antiinflationspolitik zu erzielen. Dies gilt insbesondere, wenn die politischen Erfahrungen der Wähler dagegen sprechen. Hat die gleiche Regierung z.B. schon in der Vergangenheit die Verfolgung der Preisniveaustabilisierung angekündigt und sich nicht daran gehalten bzw. vorzeitig das Ziel wieder aufgegeben, wird sie für ihr erneutes Programm keine hohe Glaubwürdigkeit erwarten dürfen. Die Wirtschaftssubjekte werden dann Zweifel am Reformwillen der Regierung haben. Selbst wenn der Regierung der Wille zur Stabilisierung zuerkannt wird, kann ihrem Programm die nötige Glaubwürdigkeit fehlen, wenn die Wähler Zweifel an der politischen Durchsetzbarkeit haben. Determinanten dafür sind die wirtschaftliche Lage der Volkswirtschaft und die Kosten der Stabilisierungsmaßnahmen. Auch für eine „linke“ Regierung ist es in einer Aufschwung- oder Boomphase nicht problematisch, eine an der Geldwertstabilität orientierte Geldpolitik an-

13 Vgl. hierzu Frey/Schneider (1978), (1979) und Schneider/Frey (1988).

14 Vgl. Hamacher (1995), S. 81-96, Kösters (1991), S. 157-160, Pfister (1981), S. $231 \mathrm{ff}$. und Willeke (1993), S. 83f. und $111 \mathrm{f}$.

15 Vgl. dazu die Untersuchungen von Roubini und Sachs (1988) und (1989). 
zukündigen und diese tatsächlich durchzuführen. Fraglich wird es in einer Rezession. Genau in dieser Phase könnte eine Regierung demonstrieren, daß sie es ernst meint mit der Inflationsbekämpfung. Die Wirtschaftssubjekte würden sie dafür mit einer steigenden Glaubwürdigkeit für ihre zukünftige Politik belohnen.

Ein Problem stellen aber die Kosten der Antiinflationspolitik dar. Stabilisierungsmaßnahmen werden in der Regel zu Beginn eines Inflationsprozesses, zu noch relativ geringen Kosten, von den Politikern noch nicht für notwendig erachtet und erst bei sichtbaren Inflationserscheinungen in Betracht gezogen. Dann jedoch können die Kosten der Inflationsbekämpfung bereits so hoch sein, daß sie politisch nicht mehr durchsetzbar ist. Insbesondere eine „linke“ Partei kann aufgrund ihrer Stammwähler der Geldwertstabilität keine Priorität beimessen, zumindest wenn gleichzeitig die Beschäftigungssituation eher negativ beurteilt wird. Eine „rechte“ Partei wird zwar annahmegemäß während der Legislaturperiode ihrer Ideologie folgend primär die Inflation bekämpfen, sobald aber der Wahlzeitpunkt in die Nähe rückt, wird sie sich nur im Falle einer sicheren Wiederwahl weiterhin für dieses Ziel einsetzen. Sollte sie hingegen am Machterhalt zweifeln müssen, wird sie sich am Wechselwähler orientieren und gegebenenfalls das Inflationsziel aufgeben.

Ein weiteres Problem der Inflationsbekämpfung ergibt sich aus dem zeitlichen Auseinanderfallen von stabilitätspolitischen Anstrengungen und Erfolg dieser Maßnahmen. Da in der Regel zunächst Mengenreaktionen erfolgen, bevor die positiven Preiswirkungen realisiert werden können, müssen Geldpolitiker einen längerfristigen Horizont haben, als dies für Politiker der Fall ist, da sie sich regelmäßig demokratischen Wahlen stellen müssen. ${ }^{16}$

Abschließend ist festzuhalten, daß viele unterschiedliche Effekte, die ihre Ursache im politischen System haben, auf die Glaubwürdigkeit der Geldpolitik und damit auf die Inflationsrate wirken. Da die Geldpolitik zumindest kurzfristig nutzbar ist, um positive Wohlfahrtseffekte für die Volkswirtschaft zu erzielen, ist nicht damit zu rechnen, daß eine permanente, am Geldwert orientierte Antiinflationspolitik von Politikern durchgeführt wird.

\subsection{2 „Strategische“ Glaubwürdigkeitsprobleme}

Probleme für eine Antiinflationspolitik resultieren aber auch aus dem ökonomischen System, wie im folgenden gezeigt wird. Betrachtet man die ökonomi-

16 Vgl. Funke (1991), S. 178-180, Issing (1992b), S. 53, Kirsch (1982), S. 38, Masson/ Symansky (1991), S. 11 und Puchta (1981), S. 106. 
schen Prozesse als Ergebnis der Interaktion zwischen dem Privatsektor und einer politischen Instanz, spielen Informationen und das strategische Verhalten hierfür eine wesentliche Rolle. Wie im zweiten Kapitel gezeigt wurde, gelingt es einer mit diskretionärer Macht ausgestatteten Regierung nicht, eine optimale Geldpolitik zu verwirklichen.

Obwohl das Problem im Rahmen des wohlfahrtstheoretischen Ansatzes diskutiert wurde und die Regierung demnach die Wohlfahrt der Bevölkerung maximieren will, ist die optimale Geldpolitik zeitinkonsistent, weil die Politiker zwei Ziele gleichzeitig anstreben. Sobald die Wirtschaftssubjekte eine Nullinflation erwarten würden, hätte die Regierung einen Anreiz, eine höhere Inflationsrate zu realisieren und dies „zum Wohle“ der Bevölkerung, da so die gesamtwirtschaftliche Wohlfahrt steigt bzw. im Rahmen des zugrunde liegenden Modells die volkswirtschaftlichen Kosten gesenkt werden können. Da die Wirtschaftssubjekte diesen Anreiz aber kennen, werden sie die Nullinflation nicht erwarten, weil sie ihren Nutzen maximieren, indem sie ihren Erwartungsfehler minimieren. Mit Hilfe der folgenden vier Gleichungen kann dies einfache Modell beschrieben werden: 17

$$
\begin{aligned}
& y=y_{n}+\left(\Pi-\Pi^{e}\right) \\
& Z=\Pi^{2}-2 \cdot\left(y-y_{n}\right) \\
& Z=\Pi^{2}-2 \cdot\left(\Pi-\Pi^{e}\right) \\
& Z_{W S}=\left(\Pi-\Pi^{e}\right)^{2}
\end{aligned}
$$$$
\text { Zielfunktion der Politiker }
$$$$
\text { Zielfunktion der Wirtschaftssubjekte }
$$

Entsprechend der ersten Gleichung kann das aktuelle Outputniveau (y) mittels einer Überraschungsinflation über das natürliche Niveau $\left(\mathrm{y}_{\mathrm{n}}\right)$ ansteigen. Dieser höhere Output beeinflußt die volkswirtschaftliche Wohlfahrt positiv und geht somit negativ in die Kostenfunktion (3.5) der Politiker bzw. der Gesellschaft ein. Durch Einsetzen der Gleichung (3.4) in Gleichung (3.5) ergibt sich die dritte Gleichung, die die Abhängigkeit der volkswirtschaftlichen Kosten von der Inflation bzw. den Inflationserwartungen darstellt. Die vierte Gleichung bringt die Kosten der Wirtschaftssubjekte zum Ausdruck, die ihren Nutzen gemindert sehen, sobald sie einem Erwartungsirrtum unterliegen. 18 Mit Hilfe dieser Gleichungen lassen sich die folgenden beiden Kostentabellen erstellen:

17 Im wesentlichen entspricht dieser Ansatz dem von BG, nur werden hier die Gleichungen vereinfacht, indem $a=b=2$ gesetzt werden. 
Tabelle 3.1: Kosten der Politiker bei Sicherheit

\begin{tabular}{c|cc} 
& \multicolumn{2}{|c}{ Wirtschaftssubjekte: $\Pi^{\mathrm{e}}=$} \\
Politiker: $\Pi=$ & 0 & 1 \\
\hline 0 & 0 & 2 \\
1 & -1 & 1
\end{tabular}

Tabelle 3.2: Kosten der Wirtschaftssubjekte bei Sicherheit

\begin{tabular}{c|cc} 
& \multicolumn{2}{|c}{ Wirtschaftssubjekte: $\Pi^{\mathrm{e}}=$} \\
Politiker: $\Pi=$ & 0 & 1 \\
\hline 0 & 0 & 1 \\
1 & 1 & 0
\end{tabular}

Die beiden Tabellen bringen die Kosten der Politiker (3.1) bzw. der Wirtschaftssubjekte (3.2) bei alternativer Wahl der Strategievariablen zum Ausdruck. Annahmegemäß setzen die Politiker die Inflationsrate und die Wirtschaftssubjekte die Inflationserwartungen fest. Wie Tabelle 3.1 zeigt, ist für die Regierung die Alternative der positiven Inflationsrate $(\Pi=1)$ die dominante Strategie, da sie unabhängig davon, was die Wirtschaftssubjekte setzen, immer die geringeren Kosten verursacht.

Da die Wirtschaftssubjekte die Anreizstruktur der Politiker kennen, wissen sie, daß die Politiker nie die Nullinflationsrate setzen werden und wählen zur Minimierung ihrer Kosten ihre Erwartung entsprechend $\left(\Pi^{\mathrm{e}}=1\right)$. In dieser Situation tragen sie Kosten von Null, während die Regierung Kosten in Höhe von Eins tragen muß. Diese Kosten, die zugleich Wohlfahrtskosten für die Gesellschaft darstellen, könnten auf Null reduziert werden, ohne daß sich die Kosten für die Wirtschaftssubjekte ändern müßten. Es liegt also keine Pareto-optimale Lösung vor. Diese, die eine erwartete und realisierte Inflationsrate von Null erfordert, könnte nur bei Kooperation erzielt werden. Die Politiker müßten sich dafür wirksam und damit glaubhaft an diese Regel binden können. Solange sie aber diskretionäre Macht besitzen, ist eine solche Regelbindung nicht glaubwürdig und damit die Pareto-optimale Lösung nicht erreichbar. ${ }^{19}$

und Wirtschaftssubjekte kommt, kann damit begründet werden, daß die Politiker sich für besser informiert halten und deshalb meinen, besser zu wissen, was gut fur das Volk ist. Vgl. Francke (1990), S. 145, FN 21. Eine alternative Erklärung ist in der Existenz externer Effekte zu sehen. Vgl. Kapitel 2.1. 
Das oben beschriebene Glaubwürdigkeitsproblem resultiert aus der Annahme symmetrischer Informationen, also einer Situation vollkommener Sicherheit. Die Wirtschaftssubjekte kennen hier nicht nur die Anreizstruktur, sondern auch die Präferenzen der Politiker. Innerhalb solch eines Rahmens ist aber kein Platz für Reputationsüberlegungen, wie sie nun vorgenommen werden sollen. Damit überhaupt ein Anreiz zum Aufbau von Reputation vorhanden ist, müssen asymmetrische Informationen vorliegen, d.h. die Politiker müssen einen Informationsvorteil haben, wie es auch realistisch ist. Dieser Informationsvorteil bezieht sich hier auf die Präferenzen der Politiker bezüglich der beiden Ziele. Zudem wird die Wohlfahrtsorientierung der Politiker aufgegeben und durch die Eigennutzhypothese ersetzt. Im weiteren Verlauf wird also der Ansatz der Neuen Politischen Ökonomie unterstellt.

Wieder wird die bekannte Zielfunktion betrachtet, die nun nicht zu vereinfachen ist:

$$
\mathrm{Z}=\frac{\mathrm{a}}{2} \cdot \Pi^{2}-\mathrm{b} \cdot\left(\Pi-\Pi^{\mathrm{e}}\right)
$$

Bei Unterstellung dieser Zielfunktion ergibt sich die folgende Kostentabelle für die Politiker:

Tabelle 3.3: Kosten der Politiker bei Unsicherheit

\begin{tabular}{c|cc} 
Politiker: $\Pi=$ & 0 & 1 \\
\hline 0 & 0 & $\mathrm{~b}$ \\
1 & $\frac{\mathrm{a}}{2}-\mathrm{b}$ & $\frac{\mathrm{a}}{2}$
\end{tabular}

Entsprechend der modifizierten Modellversion nehmen die Parameter $a$ und $b$ Werte zwischen Null und Eins an, und ihre Summe muß Eins ergeben. Man kann nun nicht mehr sofort entscheiden, welche für die Politiker die dominante Strategie darstellt. Dies ist von ihren Präferenzen für die Inflationsbekämpfung (a) bzw. die Arbeitslosigkeitsbekämpfung ( $b=1-a)$ abhängig. Nur wenn die Ungleichung $\mathrm{a} / 2<\mathrm{b}$ erfüllt ist, kann auch weiterhin davon ausgegangen werden, daß eine positive Inflationsrate hier die dominante Strategie ist. Dann führt diese Strategie unabhängig von der Wahl der Inflationserwartungen wieder zu den geringsten Kosten. Dies ist solange der Fall, wie a einen Wert unter 0,67 annimmt. Sollte die Präferenz zur Inflationsvermeidung aber größer als 0,67 (und damit relativ hoch) sein, wird die Nullinflation zur dominanten Strategie. Das Glaubwürdigkeitsproblem für eine strikte Antiinflationspolitik resul- 
tiert hier also aus der Unsicherheit der Wirtschaftssubjekte. Es liegen asymmetrische Informationen vor, weil die Wirtschaftssubjekte den genauen Wert für a nicht kennen.

Dieser spieltheoretische Nachweis zeigt, daß die Pareto-optimale Lösung nicht automatisch verfehlt wird, wenn zwei Ziele verfolgt werden. Wichtiger ist die stärkere Bewertung des Inflationsziels. Wie im vorherigen Abschnitt aber gezeigt wurde, ist dies permanent weder von einer "linken“ noch einer „rechten“ Regierung bzw. von einer von der Regierung abhängigen Zentralbank zu erwarten. Nur eine unabhängige Zentralbank, die eine hohe Reputation wünscht, wird dieser Forderung gerecht.

In der Literatur werden zwei Modelltypen zur Lösung des Glaubwürdigkeitsproblems über die Reputation diskutiert. Beide gehen von asymmetrischen Informationen aus, die sich wieder auf die unterschiedliche Zielgewichtung beziehen. Während in der ersten Modellvariante aber die Zielgewichtung zeitinvariant ist, geht die zweite davon aus, daß die Präferenzen der Geldpolitiker sich über die Zeit ändern und somit immer ein Informationsvorsprung der Politiker gegenüber den Wirtschaftssubjekten existiert. Entsprechend der Annahme der rationalen Erwartungen kennen die Wirtschaftssubjekte die Anreizstrukturen der Politiker bei Unsicherheit über deren Zielpräferenzen.

\section{a) Zeitinvariante Präferenzen}

Im Rahmen dieser Modelle ändern die Politiker ihre Präferenzen bezüglich der Zielgewichtung nicht. Die Wirtschaftssubjekte sammeln anhand der Zielrealisationen Erfahrungen über die Einstellung der Geldpolitiker, die sie in ihren zukünftigen Erwartungen berücksichtigen. Es werden meist zwei Typen von Politikern unterstellt: der absolute Inflationsgegner, der eine Nullinflation realisieren will und als ,hart" bezeichnet wird, und der Typ, dem auch die Beschäftigung wichtig ist. Diese, als „weich“ bezeichneten, Politiker, werden eine positive Inflationsrate realisieren. Reputation verdienen hier nur die „harten“ Politiker.

Da die Höhe der Reputation aber die Höhe einer Überraschungsinflation bestimmt, hat der „weiche“ Typ ebenfalls einen Anreiz, sich zunächst Reputation aufzubauen, um am Ende seiner Legislaturperiode einen möglichst hohen Ertrag in Form von sinkender Arbeitslosigkeit zu erzielen. Die Modelle unterstellen demnach einen begrenzten Zeithorizont. Da die Wirtschaftssubjekte zu Beginn einer Amtszeit nicht mit Sicherheit wissen, welcher Art von Politikern sie sich gegenübersehen, werden sie beide Möglichkeiten bei ihrer Erwartungsbildung berücksichtigen. Es gilt: 


$$
\Pi^{\mathrm{e}}=\alpha \cdot \Pi^{\mathrm{H}}+(1-\alpha) \cdot \Pi^{\mathrm{W}} \text { mit: } 0 \leq \alpha \leq 1
$$

Der Gewichtungsfaktor $\alpha$ bringt die Wahrscheinlichkeit für einen "harten“ Politiker zum Ausdruck, der mit der Inflationsrate eines „harten“ Politikers $\left(\Pi^{\mathrm{H}}\right)$ multipliziert wird. Entsprechend wird die Inflationsrate des „weichen“ Typs $\left(\Pi^{\mathrm{W}}\right)$ mit der Gegenwahrscheinlichkeit $(1-\alpha)$ gewichtet. Die Wahrscheinlichkeit $\alpha$ bringt hier die Reputation des Politikers zum Ausdruck. Bei einer hohen Reputation ist $\alpha$ nahe seinem Maximalwert Eins. Trauen die Wirtschaftssubjekte dem Politiker hingegen keine Politik zu, die an der Geldwertstabilität orientiert ist, wird $\alpha$ nahe bei Null liegen. Solange es nicht zur Inflation kommt, wird $\alpha$ von Periode zu Periode steigen, d.h. eine Antiinflationspolitik wird mit einer steigenden Reputation belohnt. Sobald jedoch nur einmal inflationiert wird, nimmt $\alpha$ den Wert Null an und behält ihn bis zum Ende des Spiels. Der Politiker besitzt dann keine Reputation mehr, und die Wirtschaftssubjekte erwarten mit Sicherheit die ,schlechte" Inflationsrate des „weichen“ Typen. Die Veränderungen von $\alpha$ spiegeln in diesen Modellen also den Lernprozeß der Wirtschaftssubjekte wider.

Aber auch der „harte“ Typ hat einen Anreiz, den Wirtschaftssubjekten möglichst schnell zu signalisieren, daß er ein Inflationsgegner ist, da die Kosten einer Inflationsbekämpfung ebenfalls von der Höhe der Reputation abhängig sind. Die Wirtschaftssubjekte und der "harte“ Politiker kennen aber andererseits den Anreiz des „weichen“ Typen zur Täuschung, und deshalb muß das Signal stark genug sein, damit er als „harter“ Typ erkannt wird. ${ }^{20}$ Nur wenn das Signal stark genug ausfällt, was unter Umständen eine erhebliche Anfangsrezession bedeutet, können die Wirtschaftssubjekte davon ausgehen, daß der „weiche" Typ nicht bereit wäre, solche hohen Kosten für die Investition in seine Reputation zu tragen. ${ }^{21}$

Das Ergebnis dieser Modelle ist, daß die Reputation das Glaubwürdigkeitsproblem zwar mindern kann, dies unter Umständen aber zu erheblichen Kosten in

Signalisierung im Rahmen der Spieltheorie bedeutet, daß der Spieler mit den privaten Informationen den ersten Schritt macht. Dies steht im Gegensatz zur bloßen Ankündigung, bei der nur die Absicht zu einem bestimmten Schritt bekundet, dieser tatsächlich aber noch nicht durchgeführt wird.

21 Zum Anreiz zur Täuschung durch den „weichen“ Typen vgl. Barro (1986) und Backus/Driffill (1985a). Die Signalisierung des „harten“ Typen kommt bei Vickers (1986) zum Ausdruck. Die Modelle basieren auf den industrieökonomischen Ansätzen von Kreps/Wilson (1982a und b) bzw. Milgrom/Roberts (1982a und b). Ein Modell, in dem beide Typen einen Anreiz zum Signalisieren haben, ist das von Mino/Tsutsui (1990). 
Form einer starken Rezession. Man könnte mit Hilfe dieser Modelle aber zu einem günstigeren Ergebnis gelangen, wenn dafür gesorgt wird, daß die Wirtschaftssubjekte schon zu Beginn des Spiels von einer hohen Anfangsreputation ausgehen. So könnten institutionelle Vorkehrungen dazu führen, daß die Geldpolitiker dem Stabilitätsziel eine hohe Gewichtung zuordnen „müssen“ und daß andererseits der Zeithorizont gegen unendlich geht. Beide Bedingungen können wieder nicht von wahlabhängigen Politikern erfüllt werden, wenn auch die zweite Bedingung insofern als erfüllt betrachtet werden könnte, als den Politikern die Zeit nach ihrer eigenen Amtszeit nicht egal ist, wie in der ersten Modellvariante unterstellt, sondern sie als Angehörige einer Partei auch an diese denken müssen und dafür Sorge zu tragen haben, daß sie wiedergewählt wird. ${ }^{22}$

Wichtiger als der größere Zeithorizont ist aber die sichtbare Präferenz für das Stabilitätsziel, die einem Politiker nicht zu jedem Zeitpunkt unterstellt werden kann. Dies könnte wieder nur eine von der Regierung völlig unabhängige Zentralbank, die sich entsprechenden Anreizen zur Verfolgung der Geldwertstabilität gegenübersieht. Wenn die Wirtschaftssubjekte davon ausgehen können, daß diese Anreize, etwa in Form negativer Sanktionen für die Zentralbank, im Falle einer Inflationierung auch greifen, könnten sie der Zentralbank bedenkenlos eine hohe Anfangsreputation zuerkennen, und eine Anfangsrezession wäre nicht erforderlich. Durch eine entsprechende institutionelle Ausgestaltung könnten also die Kosten einer Inflationsbekämpfung verringert und gleichzeitig die Unsicherheit der Wirtschaftssubjekte beseitigt, zumindest aber eingeschränkt werden.

Wenn diese Unsicherheit nicht mehr vorhanden wäre, wären die schlechteren Ergebnisse des nächsten Modells für die Reputation auch hinfällig. Dennoch soll es kurz beschrieben werden, da es sich um das realistischere Modell handelt, wenn Politiker die Geldpolitik bestimmen.

\section{b) Zeitvariante Präferenzen}

Innerhalb dieses Modells wird davon ausgegangen, daß die Politiker nicht über die gesamte Zeit die gleichen Präferenzen für ihre anzustrebenden Ziele haben. Die Präferenzen können sich von Periode zu Periode ändern, und deshalb wird die Unsicherheit der Wirtschaftssubjekte nie völlig beseitigt, obwohl sie aus dem vergangenen Verhalten wieder lernen können. Der geldpolitische Kurs wird hier von unterschiedlichen Interessen beeinflußt. Je nach Machtverhältnissen spiegeln die geldpolitischen Präferenzen die Meinung der verschiedenen

Vgl. Driffill (1988), S. 538. 
politischen Parteien, Interessenverbände oder Gewerkschaften wider. Die Stärke der Einflußnahme ist vom Ausmaß der Unabhängigkeit der Zentralbank abhängig. Nur wenn die Geldpolitik ,entpolitisiert" wird, indem die Zentralbank völlig weisungsungebunden ist, braucht die Geldpolitik dem äußeren Druck nicht nachzugeben. Auch dieses Modell führt also wieder zum Ergebnis, daß die Unabhängigkeit der Zentralbank die Glaubwürdigkeit begünstigt, welche dann zu sinkenden Inflationserwartungen und somit geringeren Kosten der Inflationsbekämpfung führt. ${ }^{23}$

Eine Erweiterung erfährt dies Modell noch um die Nutzung von Ankündigungen als Instrument der Geldpolitik, die die Unsicherheit der Wirtschaftssubjekte senken sollen. Der Vorteil einer Ankündigung wäre auch, daß sie im Gegensatz zur Signalisierung keine Kosten verursacht. Während zur Signalisierung zunächst tatsächlich eine geringe Inflationsrate gesetzt werden muß, die aufgrund der höheren Erwartungen zur Rezession führt, kann diese bei einer Ankündigung ausbleiben, da die Wirtschaftssubjekte ihre Erwartungen vorher an die beabsichtigte, geringere Inflationsrate anpassen können. Bedingung für die Erwartungsminderung ist jedoch, daß die Ankündigung von den Wirtschaftssubjekten auch geglaubt werden muß, und dies wiederum ist abhängig von der Reputation, die eine Autorität schon besitzt.

Wenn ein Politiker im Extremfall noch keine Reputation besitzt, werden die Wirtschaftssubjekte nicht allein aufgrund der Ankündigung einer stabilitätsorientierten Geldpolitik ihre Inflationserwartungen senken. Dies Instrument ist also erst nutzbar, wenn der Politiker schon eine gewisse Reputation besitzt oder in langer Frist. So werden die Wirtschaftssubjekte Ankündigungen glaubwürdig finden, wenn sie in der Vergangenheit feststellen konnten, daß die Politiker sich an ihre Ankündigungen gehalten haben. Dann steigt tatsächlich die Planungssicherheit für die Wirtschaftssubjekte durch die Ankündigung, da sie sich nun nicht mehr ausschließlich auf die vergangenen Realisierungen der Geldpolitik stützen müssen, sondern auch aktuelle Signale über den Kurs der Geldpolitik berücksichtigen können. Die Unsicherheit der Wirtschaftssubjekte nimmt um so mehr ab, je glaubwürdiger die Ankündigungen sind, und desto schneller werden sie ihre Erwartungen anpassen.

Die Glaubwürdigkeit der Ankündigung ist auch abhängig von ihrer Präzision. Je unpräziser, z.B. in Form von Bandbreiten, sie vorgenommen wird, desto glaubwürdiger ist sie zwar, aber gleichzeitig nimmt auch ihr Informationsgehalt

23 Auf eine weiterführende Ausführung dieses Modells soll hier verzichtet werden, weil letztlich nur das Ergebnis interessiert. Zur detaillierten Analyse vgl. Cukierman/ Meltzer (1986b) und Cukierman (1992), S. 165ff. 
ab. Damit die Ankündigung für die Wirtschaftssubjekte nutzbar ist, d.h. ihre Unsicherheit sinken kann, muß immer der Informationsgehalt berücksichtigt werden. Andererseits hat eine Ankündigung selbst auch einen disziplinierenden Effekt auf den Politiker, da er weiß, daß unzuverlässige Ankündigungen die Glaubwürdigkeit und damit dann seine Reputation schnell wieder mindern können.

$\mathrm{Zu}$ berücksichtigen ist aber auch, daß dies Instrument von einem wahlabhängigen Politiker dazu genutzt werden könnte, die Wähler bewußt zu täuschen. Langfristig wirksam dürfte dies Instrument deshalb auch wieder nur sein, wenn eine unabhängige Zentralbank es anwendet, mit den entsprechenden Anreizen zur Stabilitätspolitik. Nur in diesem Falle können die Wirtschaftssubjekte davon ausgehen, daß das Instrument nicht irgendwann wahltaktisch genutzt wird. ${ }^{24}$

Alle strategischen wie auch politischen Glaubwürdigkeitsprobleme sprechen bisher für die Installierung einer unabhängigen Zentralbank, da nur diese eine hohe Reputation mit allen positiven Wirkungen erzielen kann. Genau diese institutionelle Trennung zwischen Geld- und Wirtschaftspolitik kann aber zu einem anderen Glaubwürdigkeitsproblem führen, wenn das gesamte makroökonomische Programm inkonsistent ist, weil die Regierung eine expansive Fiskal- und die Zentralbank eine restriktive Geldpolitik verfolgen will.

Bei dieser unterschiedlichen Politikausrichtung ist das Ziel der Preisniveaustabilisierung nicht voll glaubwürdig. Die Gegner einer unabhängigen Zentralbank argumentieren eben mit diesem Koordinationsproblem, welches letztlich zu Effizienzverlusten für die Wirtschaft führt. Das Gegenteil ist aber der Fall, wenn man einen stabilen Geldwert garantieren will, da nur eine tatsächlich unabhängige Zentralbank die Regierung dann dominieren und disziplinieren kann. ${ }^{25}$

\subsection{3 „Technologisches“ Glaubwürdigkeitsproblem}

Unabhängig davon, ob ein Politiker oder eine unabhängige Zentralbank die Geldpolitik lenkt, tritt noch ein zusätzliches Glaubwürdigkeitsproblem auf,

24 Zur analytisch ausführlichen Darstellung der Ankündigungseffekte vgl. Cukierman/ Meltzer (1986b). Die Nichtbeachtung von Ankündigungen abhängiger Zentralbanken bringt Neumann (1992), S.66 zum Ausdruck.

25 Vgl. dazu den grundlegenden Artikel von Sargent/Wallace (1981). Kooperation zieht immer eine Kompromißlösung nach sich. So könnte ein „,bißchen“ Inflation zugelassen werden, um die wirtschaftliche Entwicklung nicht zu behindern. Genau dies widerspräche aber den empirischen Ergebnissen. Vgl. hierzu auch Hasse (1990), S. 75. 
welches die Literatur zum Zeitinkonsistenzproblem nahezu komplett vernachlässigt. Da die meisten Modelle von der Annahme der perfekten Kontrolle der Inflation oder des Geldangebots durch die Zentralbank ausgehen, wurde dies Problem ausgeklammert.

Eine Modellvariante berücksichtigt zwar die unzureichende Kontrolle des Geldangebots durch die geldpolitische Instanz, hinterfragt aber nicht weiter die Ursachen dessen, sondern begründet dies mit Zufallseinflüssen. ${ }^{26}$ Gerade aufgrund der Ergebnisse dieses Modells sind die Ursachen der Nicht-Kontrollierbarkeit der Geldmenge aber zu erkunden. Das Fazit lautet, daß bei Vorliegen von Kontrollfehlern des Geldangebots die Wirtschaftssubjekte die vergangene Leistung der Zentralbank weniger berücksichtigen für ihre Prognose der zukünftigen Inflationsrate. Auch die anderen Determinanten der Reputation werden eher unwichtig, da diese in zunehmendem Maße vom vorliegenden Glaubwürdigkeitsproblem dominiert wird. Anhand des Zeitpfades in Abbildung 3.1 kann das Problem veranschaulicht werden:

Abbildung 3.1: Inflationserwartungen, geplante und tatsächliche Inflation

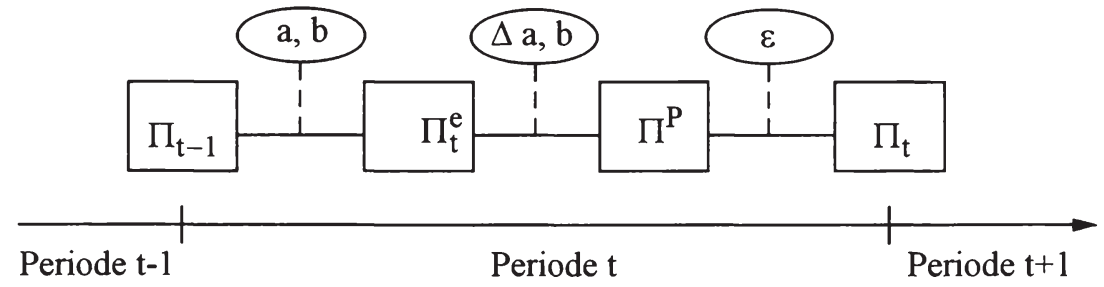

Quelle: Vgl. Kastner (1994), S. 125.

Die Wirtschaftssubjekte beobachten die realisierte Inflationsrate der Periode t-1 am Ende dieser Periode. Aufgrund dieser Beobachtung ziehen sie Rückschlüsse auf die Präferenzen ( $a$ und $b$ ) der Politiker, wie sie in der Periode t-1 ihrer Meinung nach vorlagen. Unter Berücksichtigung der vergangenen Realisation und der daraus abgeleiteten Präferenzen bilden sie ihre Inflationserwartungen für die Periode t. Erst nachdem die Wirtschaftssubjekte unter Annahme einer bestimmten Inflationsrate ihre Löhne ausgehandelt haben, planen die Politiker die Realisation von $\Pi\left(\Pi^{\mathrm{P}}\right)$, wobei sich aber aufgrund neuer Ausgangsbedingungen ihre Präferenzen für die beiden Ziele verlagert haben können. Die von den Politikern geplante Realisation der Inflationsrate kann nun wiederum durch Zufallseinflüsse $(\varepsilon)$ gestört werden, so daß tatsächlich eine „verzerrte“

Vgl. Cukierman/Meltzer (1986a und b) und Cukierman (1992). 
Inflationsrate realisiert wird. Nur diese „verzerrte“ Rate können die Wirtschaftssubjekte beobachten und werden mit Hilfe dieser Rate ihre Erwartungen bilden. Da diese Rate von den Politikern aber nicht perfekt kontrollierbar ist, sind sie nicht in der Lage, ihre Reputation allein zu verursachen, weil diese auch durch Zufallseinflüsse bestimmt wird.

Die Wirtschaftssubjekte könnten aufgrund einer hohen Realisation von $\Pi$ auf eine geringe Inflationsaversion der Zentralbank schließen, was tatsächlich aber nicht der Fall sein muß, wenn Störungen des Geldangebots zu dieser hohen Realisation geführt haben. Andererseits könnte der Fall eintreten, daß die Wirtschaftssubjekte der Zentralbank zutrauen, im Sinne der Geldwertstabilität handeln zu wollen, wegen der hohen realisierten Inflationsrate aber daran zweifeln, daß die Zentralbank die Inflationsrate aufgrund hoher Kontrollfehler überhaupt noch steuern kann. Aufgrund der fehlenden Kontrolle über die Geldpolitik kann diese nicht glaubwürdig sein, da sie in erheblichem Maße vom Zufall mitbestimmt wird. Die fehlende Glaubwürdigkeit bedingt wiederum nur eine geringe Reputation.

Zur Reputationssteigerung ist es hier notwendig, dieses "technologische“ Glaubwürdigkeitsproblem zu lösen. Den Ausgangspunkt bildet die Unwirksamkeit der Geldpolitik, womit sich als zu untersuchendes Problem der monetäre Transmissionsmechanismus ergibt, was Abbildung 3.2 verdeutlichen soll:

Abbildung 3.2: Mögliche Störungen der Geldpolitik

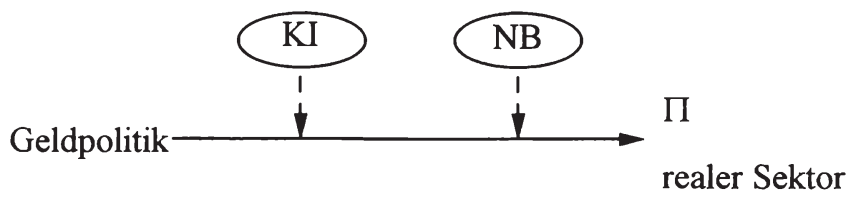

Es wird nun nicht weiterhin unterstellt, daß die geldpolitischen Impulse sofort und in gewünschter Weise auf die Inflationsrate und den realen Sektor wirken, sondern daß es im Laufe des monetären Transmissionsmechanismus zu Störungen kommt, verursacht durch das Geschäftsbankensystem (KI) oder den Nichtbankensektor (NB) ${ }^{27}$ Die Geldpolitik kann somit nur verzerrt oder sogar völlig entgegengesetzt wirken. ${ }^{28}$

27 Eventuelle Zeitverzögerungen, die auch ohne „fremdes Zutun“ effizienzmindernd auf die Geldpolitik wirken können, werden hier nicht berücksichtigt.

28 Dieser Sachverhalt wird auch durch die Bestimmung des Geldangebots (M) als $\mathrm{M}=\mathrm{m} \cdot \mathrm{B}$ zum Ausdruck gebracht. Die Zentralbank kann demnach nicht das gesamte Geldangebot allein bestimmen, sondern nur die Geldbasis (B). Der Geldmultiplikator 
Letztlich versucht die Zentralbank mit Hilfe ihres geldpolitischen Instrumentariums, das Verhalten der Akteure zu beeinflussen. Eine zentrale Rolle spielen hierbei die Finanzintermediäre, da sie als erste auf die geldpolitischen Maßnahmen reagieren und die Impulse an den Nichtbankensektor weiterleiten sollen. Demnach muß zunächst das Verhalten der Kreditinstitute untersucht werden. Dieses läßt sich aufgrund ihrer Zielfunktion ableiten. Wie jedes andere Unternehmen streben die Geschäftsbanken eine Gewinnmaximierung an. Dabei werden sie durch die Zentralbank insofern restringiert, als diese über die Zuteilung von Zentralbankgeld entscheidet, ein Medium, auf welches die Kreditinstitute bei Durchführung ihrer Geschäfte angewiesen sind und das sie nicht selbst schaffen können.

Will die Zentralbank zur Durchführung einer Antiinflationspolitik die Geldmenge mindern, muß sie dafür sorgen, daß den Kreditinstituten weniger Zentralbankgeld zur Verfügung steht. Hiermit wird die Frage nach der Verfügbarkeit eines effizienten geldpolitischen Instrumentariums aufgeworfen.

In entwickelten Marktwirtschaften spielen zunehmend die indirekten, marktkonformen Wertpapierpensionsgeschäfte die dominante Rolle. Wenn man davon ausgeht, daß genügend handelbare Papiere zur Verfügung stehen, stellt dies Instrument zur Feinsteuerung ein sehr wirksames und zugleich geräuschloses Instrument der Geldpolitik dar. Es muß jedoch berücksichtigt werden, ob nicht gerade im Hinblick auf die Bedeutung für die Reputation der Zentralbank auch das Vorhandensein möglicher Zwangsmaßnahmen, also nicht-marktkonformer Instrumente, förderlich wären. Selbst wenn der Einsatz dieses Instruments nicht nötig ist, könnte allein die Existenz disziplinierend auf die Übermittler der monetären Impulse wirken. Sollte aus irgendwelchen Gründen die Offenmarktpolitik nicht entsprechend der Intention der Zentralbank wirken können, hätte sie über die Mindestreservepolitik doch die Möglichkeit, die Kreditinstitute zu einem bestimmten Verhalten zu zwingen.

Aber auch auf die nicht so „scharfen“, dafür aber ,lauteren“ Instrumente sollte im Rahmen der geldpolitischen Möglichkeiten nicht völlig verzichtet werden, da auch sie positiv auf die Reputation wirken können. Zwar ist die Bedeutung der hier angesprochenen Refinanzierungspolitik (Diskont- und Lombardpolitik) für die Steuerung der Geldmenge erheblich zurückgegangen, trotzdem sollte ihre Signalwirkung auf das Publikum berücksichtigt werden. Wie im letzten Abschnitt dargestellt wurde, können Signale der Zentralbank ihre Reputation beeinflussen. Die Signalwirkung von Offenmarktgeschäften hat aber noch nicht

(m) beinhaltet hier das Störpotential. 
das gleiche Ausmaß erreicht wie insbesondere die Veränderung der Refinanzierungssätze. Bei einem völligen Verzicht auf dieses geldpolitische Instrument würde man freiwillig auf eine Möglichkeit der Beeinflussung der Reputation und damit der Inflationserwartungen verzichten.

Die Notwendigkeit eines sichtbaren Signals auch für die Wirtschaftssubjekte resultiert aus der Tatsache, daß auch der Nichtbankensektor einen Störfaktor für die Geldpolitik der Zentralbank darstellen kann. Selbst wenn die Kreditinstitute den geldpolitischen Impuls ,unverzerrt" weitergeleitet haben, bleibt der restriktive Effekt einer Antiinflationspolitik von seiten der Zentralbank aus, wenn die Unternehmen aufgrund fehlender Möglichkeiten zur Kreditaufnahme bei den Geschäftsbanken vermehrt dazu übergehen, sich bei offenen Kapitalmärkten im Ausland zu refinanzieren. Eine weitere Konterkarierungsmöglichkeit für die Geldpolitik kann ein entsprechendes Wechselkursregime darstellen. Besteht aufgrund der Vereinbarungen fester Wechselkurse für die Zentralbank die Pflicht zur Intervention am Devisenmarkt, kann dadurch die Effizienz ihrer heimischen Antiinflationspolitik gemindert werden.

Es bleibt festzuhalten, daß es aufgrund von Störungen des monetären Transmissionsprozesses zu ,technologischen" Glaubwürdigkeitsproblemen für eine Antiinflationspolitik kommen kann, die den Aufbau von Reputation trotz sonst günstiger Rahmenbedingungen verhindern können. Durch eine geeignete institutionelle Ausgestaltung insbesondere des geldpolitischen Instrumentariums können die möglichen Problemursachen aber zumindest teilweise beseitigt werden. Unterstützend für die Glaubwürdigkeit ist auch eine systematische Durchführung der Geldpolitik, da es den Wirtschaftssubjekten so leichter fällt, Veränderungen der Politik festzustellen. Die völlige Beseitigung der Störeinflüsse auf die Kontrollierbarkeit des Geldangebots und damit die Inflationsrate wird nicht möglich sein, da exogene Schocks nicht auszuschließen sind. Solange es sich aber tatsächlich um exogene Schocks handelt, die nur vereinzelt auftreten und von den Wirtschaftssubjekten auch als solche erkannt werden können, leidet die Reputation der Zentralbank nicht. Wenn die Wirtschaftssubjekte davon ausgehen können, daß die Zentralbank die Geldmenge im „Normalfall“ unter Kontrolle hat, werden sie die Reputation auch wieder in Abhängigkeit der anderen Determinanten wählen. ${ }^{29}$

29 Vgl. Eijffinger (1991), S. 180ff., Hamacher (1995), S. 48 und 106-118, Marlow (1991), S. 472 und Puchta (1981), S. 244 und 262. 


\subsubsection{Die Bedeutung der Glaubwürdigkeit für das Modell}

Es wurde modelltheoretisch gezeigt, daß der Aufbau von Reputation einer Zentralbank leichter fällt, wenn die Wirtschaftssubjekte ihre Geldpolitik glaubwürdig finden $\left(\Pi^{\mathrm{e}}=\Pi^{\mathrm{a}}\right)$. Die Zentralbank wird ihre Reputation dann nicht nur schneller steigern, weil sie geringere optimale Inflationsraten realisieren kann, sie muß zudem keine keine Stabilisierungskrise hierfür verursachen. Die Kosten für die Gesellschaft hängen dann nur von der realisierten Inflationsrate der Zentralbank ab. Wie im BG-Modell gilt hier:

$$
\begin{aligned}
& \Pi^{\mathrm{opt}}=\Pi^{\mathrm{a}}=\Pi^{\mathrm{e}}=\frac{\mathrm{b}}{\mathrm{a}} \cdot \frac{1-\mathrm{q}}{1+\mathrm{q}} \text { und } \\
& Z=\frac{\mathrm{a}}{2} \cdot\left(\Pi^{\mathrm{opt}}\right)^{2}
\end{aligned}
$$

Halten die Wirtschaftssubjekte die angekündigte optimale Inflationsrate der Zentralbank nicht für glaubwürdig, ist diese auch für die Zentralbank nicht mehr optimal. Sie muß dann die höheren Erwartungen der Wirtschaftssubjekte bei der Wahl ihrer Realisation berücksichtigen. Es gilt:

$$
\Pi^{\mathrm{opt}}=\Pi^{\mathrm{a}}=\frac{\mathrm{b}}{\mathrm{a}} \cdot \frac{1-\mathrm{q}}{1+\mathrm{q}}<\Pi^{\mathrm{e}}
$$

Dann muß die Zentralbank ihre optimale Inflationsrate entsprechend (2.25) berechnen:

$$
\Pi^{\mathrm{ZB}}=\frac{\mathrm{b}}{\mathrm{a}} \cdot \sqrt{\frac{2 \cdot \mathrm{a}}{\mathrm{b} \cdot(1+\mathrm{q})} \cdot \Pi^{\mathrm{e}}-\frac{1-\mathrm{q}}{1+\mathrm{q}}}
$$

Für die Realisation der Zentralbank gilt:

$$
\Pi^{\mathrm{a}}<\Pi^{\mathrm{ZB}}<\Pi^{\mathrm{e}}
$$

Sie verursacht damit die folgenden Kosten:

$$
\mathrm{Z}=\frac{\mathrm{a}}{2} \cdot \Pi^{\mathrm{ZB}}-\mathrm{b} \cdot\left(\Pi^{\mathrm{ZB}}-\Pi^{\mathrm{e}}\right) \quad \text { mit: } \Pi^{\mathrm{ZB}}<\Pi^{\mathrm{e}}
$$

Im Falle einer unglaubwürdigen Geldpolitik treten also Kosten nicht nur in Höhe der realisierten Inflationsrate auf, sondern zusätzlich noch in Höhe der 
zunehmenden Arbeitslosigkeit, die durch die Antiinflationspolitik verursacht wird.

Wie schon im Kapitel 3.1.3 gezeigt wurde, wird die optimale Inflationsrate der Zentralbank je nach Höhe der Inflationserwartungen der Wirtschaftssubjekte variieren. Je höher ihr Wert ist, desto höher wird auch die optimale Inflationsrate für die Zentralbank ausfallen, da die Kosten der Stabilisierungskrise zu berücksichtigen sind. Andererseits bedeutet dies für die Reputation, daß sie um so langsamer steigen wird. Hohe Inflationserwartungen der Wirtschaftssubjekte bedingen eine ,relativ hohe“ Realisation der Zentralbank, welche die Inflationserwartungen für die nächste Periode dann nur ,geringfügig“ mindern können. Genau in der Höhe der Inflationserwartungen kommt aber die Reputation zum Ausdruck.

Würde die Zentralbank eventuell sogar auf eine Stabilisierungskrise verzichten wollen, indem sie die Inflationserwartungen der Wirtschaftssubjekte erfüllt, würde dies für ihre Reputation bedeuten, daß sie sich nicht ändert. ${ }^{30}$ Die Wirtschaftssubjekte würden dann in der Zukunft die gleichen „hohen“ Inflationsraten erwarten und geringere Inflationsankündigungen der Zentralbank nicht glauben, selbst wenn diese es ehrlich meinen sollte. Um in solch einer Situation der unglaubwürdigen Geldpolitik die Inflationserwartungen zu brechen, ist es unabdingbar für die Zentralbank, eine Stabilisierungskrise herbeizuführen. Dabei gilt, daß sie ihre Reputation um so schneller steigern kann, je größer die Krise ausfällt und umgekehrt. Bei einer unglaubwürdigen Geldpolitik sieht die Zentralbank sich also einem Trade-off zwischen Reputationssteigerung und temporärer Steigerung der Arbeitslosigkeit gegenüber. Für die Reputation der Zentralbank wäre es demnach förderlich, wenn die Wirtschaftssubjekte die Antiinflationspolitik glaubwürdig finden würden. Nur dann gelingt der Aufbau von Reputation „kostenlos“ und ist deshalb um so wahrscheinlicher. Eine glaubwürdige Geldpolitik kann deshalb als Voraussetzung für den Aufbau von Reputation betrachtet werden. Es ist dann erforderlich, die diskutierten Glaubwürdigkeitsprobleme zu lösen.

\subsubsection{Lösung der Glaubwürdigkeitsprobleme}

Es wurde gezeigt, daß insbesondere Politiker Probleme mit einer glaubwürdigen Antiinflationspolitik haben. Um die Glaubwürdigkeitsprobleme für die Politiker zu lösen, wurde vorgeschlagen, ihre Macht einzuschränken, indem sie an eine fest vorgegebene Regel gebunden werden. Die Unsicherheit der Wirt-

30 Das war bei BG anders, da bei ihnen die Erfüllung der Erwartungen zum Reputationsaufbau führte. Vgl. auch Kapitel 2.1 und 2.2. 
schaftssubjekte könnte so sinken, weil sie wüßten, wie die Politiker handeln würden bzw. müßten. Als Problem erweist sich hierbei jedoch die Formulierung einer optimalen Regel. Eine starr vorgegebene Regel, die den Politikern keinen Spielraum mehr läßt, birgt die Gefahr, bei unvorhergesehenen Ereignissen nicht reagieren zu können. Dann würden die Politiker zwar die Erwartungen erfüllen und durch die Realisation der glaubwürdigen Geldpolitik ihre Reputation steigern, andererseits würden sie dafür eine Stabilisierungskrise verursachen. Diese könnten sie verhindern, wenn sie sich nicht an die starr vorgegebene Regel hielten und die Erwartungen nicht erfüllten. Dennoch muß diese „Nichtrealisation“ der Erwartungen nicht unbedingt zur Minderung der Reputation beitragen. Es hängt dann von der Öffentlichkeitsarbeit der Politiker ab, ob diese den Wirtschaftssubjekten ihren „Verstoß“ gegen die Regel glaubhaft begründen können. Gelingt dies, würden die Wirtschaftssubjekte eine Antiinflationspolitik auch für die nächste Periode glaubwürdig finden, da sie darauf vertrauen könnten, daß nur bei selten auftretenden unvorhersehbaren Störungen von der Regel abgewichen wird und dies auch nur, um der Volkswirtschaft eine Anpassungskrise zu ersparen.

Ließe man den Politikern aber gleich die Möglichkeit, eigenmächtig zu entscheiden, sobald eine Störung auftritt, die bei der Formulierung der Regel nicht vorgesehen wurde, könnten die Politiker letztlich doch wieder wahltaktisch handeln, indem sie sich mit dem Vorliegen einer Ausnahmesituation und deshalb erforderlichem Abweichen von der Regel entschuldigten. Eine Lösung des Glaubwürdigkeitsproblems kann eine Regelbindung allein also nicht darstellen.

Eine andere Möglichkeit der Einschränkung der diskretionären Macht der Regierung ist in der Gewaltenteilung zu sehen. Diese kann sowohl horizontal als auch vertikal erfolgen, wie Abbildung 3.3 verdeutlicht:

Abbildung 3.3: Institutionelle Machtteilungsmechanismen

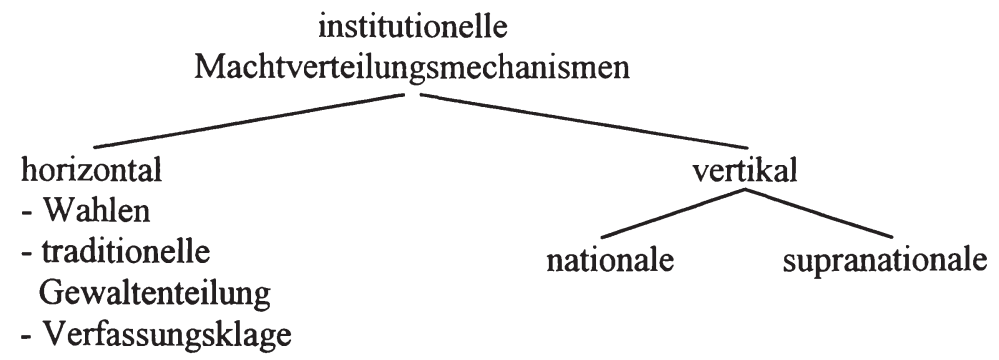

Quelle: Vgl. Hamacher (1995), S. 102. 
Untersucht man die prinzipiellen Möglichkeiten der Machtteilung für das Ziel der Geldwertstabilität und damit der Reputation, muß man die horizontalen Mechanismen als wenig wirksam einstufen. Wie gezeigt wurde, wirken Wahlen eher in die entgegengesetzte, inflationäre Richtung. Auch die traditionelle Gewaltenteilung in Exekutive, Legislative und Judikative verspricht wenig Abhilfe für das Problem der positiven Inflationsraten. Zwar kann die Exekutive nur innerhalb des Rahmens handeln, den die Legislative ihr vorgibt und dessen Einhaltung bzw. Verfassungsmäßigkeit die Judikative zu überwachen hat, in bezug auf das Ziel der Geldwertstabilität hat die Exekutive aber weitgehend freie Hand, da die Preisniveaustabilität in der Praxis nicht als einziges Ziel für die Akteure der Wirtschaftspolitik gesehen wird. Gibt die Legislative der Exekutiven aber mehrere Ziele vor, könnte letztere versuchen, ein Abweichen vom Ziel Preisniveaustabilität mit den Erfordernissen anderer, auch vorgegebener Ziele zu begründen. Für das Ziel der Geldwertstabilität ist die traditionelle Gewaltenteilung demnach nicht hilfreich. Zum gleichen Urteil kommt man bezüglich des Instituts der Verfassungsklage, da das öffentliche Gut, Vermeidung von Inflation, nicht einklagbar ist. Selbst wenn dies theoretisch möglich wäre, würde kaum ein Wirtschaftssubjekt die privaten Kosten einer Klage auf sich nehmen, um die Realisation eines Nutzens durchzusetzen, der der gesamten Währungsgemeinschaft zugute käme.

Erfolgversprechender sind die vertikalen Machtteilungsmechanismen. Die Regierung hat die Möglichkeit, Macht nach unten oder oben abzugeben. Die erste Möglichkeit erfolgt auf der nationalen Ebene und meint zunächst den föderalistischen Staatsaufbau. Da die Geldpolitik innerhalb eines Landes effizient aber nur zentralistisch durchgeführt werden kann, muß hier die Delegation an nur eine unabhängige Instanz gemeint sein. Demnach könnte das Glaubwürdigkeitsproblem der Politiker für eine am Geldwert orientierte Geldpolitik gelöst werden, indem sie diese Aufgabe an eine unabhängige Zentralbank delegieren. Die Bindung der Regierung ist jedoch nur wirksam, wenn dadurch die Macht der Regierung tatsächlich abnimmt, d.h. sie darf nicht in der Lage sein, diese Selbstbindung wieder aufzuheben. Unter der Annahme, daß Zentralbank und Politiker unterschiedliche Präferenzen haben, kommen die in der Literatur diskutierten theoretischen Modelle zum gleichen Ergebnis, unabhängig davon, ob die Geldpolitiker ihre Präferenzen über die Zeit beibehalten oder wechseln.

Eine andere Möglichkeit für die Regierung, ihre Macht einzuschränken, besteht in der Wahl der internationalen Lösung. Das Glaubwürdigkeitsproblem könnte gelöst werden, indem die Macht über die Geldpolitik an supranationale Organisationen abgegeben wird. Die Wahl fester Wechselkurse kann eine erhebliche Begrenzung der diskretionären Macht der inländischen Regierung über die 
Geldpolitik darstellen. Man kann in diesem Fall von einem Glaubwürdigkeitsimport sprechen, vorausgesetzt die Währung bzw. die Zentralbank, an die man sich bindet, besitzt einen entsprechenden Ruf. ${ }^{31}$ Eine abgeschwächte Variante dieser Politik ist die informelle Bindung an einen Stabilitätsanker. Bei dieser Lösung wird die Macht der inländischen Regierung nicht so stark begrenzt, da es keine formellen Vereinbarungen gibt und somit auch keine Pflicht zur Intervention. Andererseits wird die Glaubwürdigkeit der Politik entsprechend langsamer steigen, da die latente Gefahr der Aufgabe dieser Bindung besteht. Die Delegation nach oben ist allerdings eher für kleine Volkswirtschaften möglich. ${ }^{32}$ Deshalb bleibt großen Volkswirtschaften letztlich nur die Möglichkeit, die Geldpolitik an eine unabhängige Zentralbank abzugeben, damit das Glaubwürdigkeitsproblem der Politiker gelöst werden kann.

\subsection{Die Unabhängigkeit einer Zentralbank \\ 3.3.1 Unabhängigkeit als notwendige, aber nicht hinreichende Bedingung}

Oft wird die Installierung einer unabhängigen Notenbank in der Literatur als notwendige und schon hinreichende Bedingung zur Erreichung des Ziels der Preisniveaustabilität gesehen. Untermauert wird diese Aussage von einer Vielzahl empirischer Untersuchungen, die die negative Korrelation zwischen Inflation und Unabhängigkeit bestätigen. Des weiteren negieren sie auch den negativen Zusammenhang zwischen Inflation und Arbeitslosenrate, da Länder mit hohen Inflationsraten auch hohe Arbeitslosenquoten aufweisen. Eine Politik des stabilen Geldes ist somit die beste Voraussetzung für ein hohes Beschäftigungsniveau und ein gleichmäßiges Wirtschaftswachstum. ${ }^{33}$

31 Vgl. zur nationalen Delegation Rogoff (1985), der die Wahl „konservativer“ Zentralbankpräsidenten fordert, also Akteuren, die das Inflationsziel mehr betonen als das Beschäftigungsziel, oder alternativ die Vorgabe konkreter Anreize für die Zentralbank ein bestimmtes, eng definiertes Ziel einzuhalten. Zur internationalen Delegation vgl. Giavazzi/Pagano (1988), die den Beitritt zum EWS mit dem Glaubwürdigkeitsimport begründen, genau wie die wohlfahrtstheoretischen Analysen von Giavazzi/Giovannini (1989) und Melitz (1988).

32 Vgl. Brunetti (1992), S. 100-106, Collignon (1991), S. 119f., Eijffinger (1991), S. 179, Hamacher (1995), S. 102-104, Kastner (1994), S. 205 und 226 sowie Neumann (1992), S. 68.

33 Vgl. Filc (1994), S. 699 und 701 und Woll (1991), S. 166f. Eine Übersicht empirischer Studien zur Notenbankunabhängigkeit findet sich bei Kastner (1994), S. 231235. Vgl. auch Hasse (1989a), S. 127f. 
Allerdings vernachlässigen die empirischen Untersuchungen meist die Differenzierung zwischen formaler und faktischer Unabhängigkeit. ${ }^{34}$ Gleiches gilt für die theoretische Diskussion um die Unabhängigkeit, da der größte Teil der Unabhängigkeitsliteratur ausschließlich den Aspekt der rechtlichen Unabhängigkeit zum Untersuchungsgegenstand hat. Tatsächlich muß neben der rechtlichen auch die faktische Unabhängigkeit hinterfragt werden, die als informelle Macht gesehen werden kann. Aber selbst wenn eine Zentralbank sowohl in rechtlicher als auch formaler Hinsicht unabhängig ist, strebt sie nicht automatisch das Ziel Preisniveaustabilität an. Eine Zentralbank handelt nicht ex definitione im Interesse des Gemeinwohls, sondern auch sie verfolgt primär ihre eigenen Ziele ${ }^{35}$, die sich nur unter ganz bestimmten Umständen mit dem Allgemeinwohl decken. Bevor Aussagen über die Realisierungschancen des Ziels Geldwertstabilität getroffen werden können, müssen also die möglichen Ziele der Zentralbanker untersucht werden. Es ist zu hinterfragen, ob der Aufbau von Reputation ein primäres persönliches Ziel eines Zentralbankers ist.

Ist dies nicht der Fall, dann ist die Unabhängigkeit zwar eine notwendige, aber noch keine hinreichende Bedingung für die Geldwertstabilität. Als institutionelle Verselbständigung des Ziels der Geldwertstabilität wäre eine unabhängige Zentralbank zu sehen, die gesetzlich auf ein Ziel, die Preisniveaustabilität, verpflichtet wäre. Die Unabhängigkeit der Zentralbank wäre dann eine funktionsbezogene und würde das eigentliche Ziel, den Aufbau von Reputation, mehr in den Vordergrund stellen. ${ }^{36}$

\subsubsection{Bestimmungsfaktoren der rechtlichen Unabhängigkeit}

Unabhängigkeit, wie sie hier gefordert wird, soll nun anhand ihrer Bestimmungsfaktoren diskutiert werden. ${ }^{37}$ Die Zentralbank sollte in funktioneller, inklusive instrumenteller, personeller und finanzieller Hinsicht von der Regierung unabhängig sein, wie Abbildung 3.4 veranschaulicht.

34 Vgl. Filc (1994), S. 702. Eine sehr umfangreiche Untersuchung neueren Datums, die diese Differenzierung vornimmt, ist die von Cukierman (1992).

35 Dies klingt schon bei Eucken an: „...die Erfahrung zeigt, daß eine Währungsverfassung, die den Leitern der Geldpolitik freie Hand läßt, diesen mehr zutraut als ihnen im allgemeinen zugetraut werden kann. Unkenntnis, Schwäche gegenüber Interessengruppen und der öffentlichen Meinung, falsche Theorien, alles das beeinflußt diese Leiter sehr zum Schaden der ihnen anvertrauten Aufgabe." Eucken (1990), S. 257. Vgl. Filc (1994), S. 700, Kirsch (1982), S. 43 und Kösters (1990), S. 128f.

37 Zu möglichen Definitionen dieses Begriffs vgl. Caesar (1981), S. 56-62. Teilweise wird in der neueren Literatur der von Hansmeyer (1968), S. 155ff. geprägte Begriff „Handlungsspielraum“ statt „Unabhängigkeit“ verwendet. 
Abbildung 3.4: Komponenten der rechtlichen Unabhängigkeit

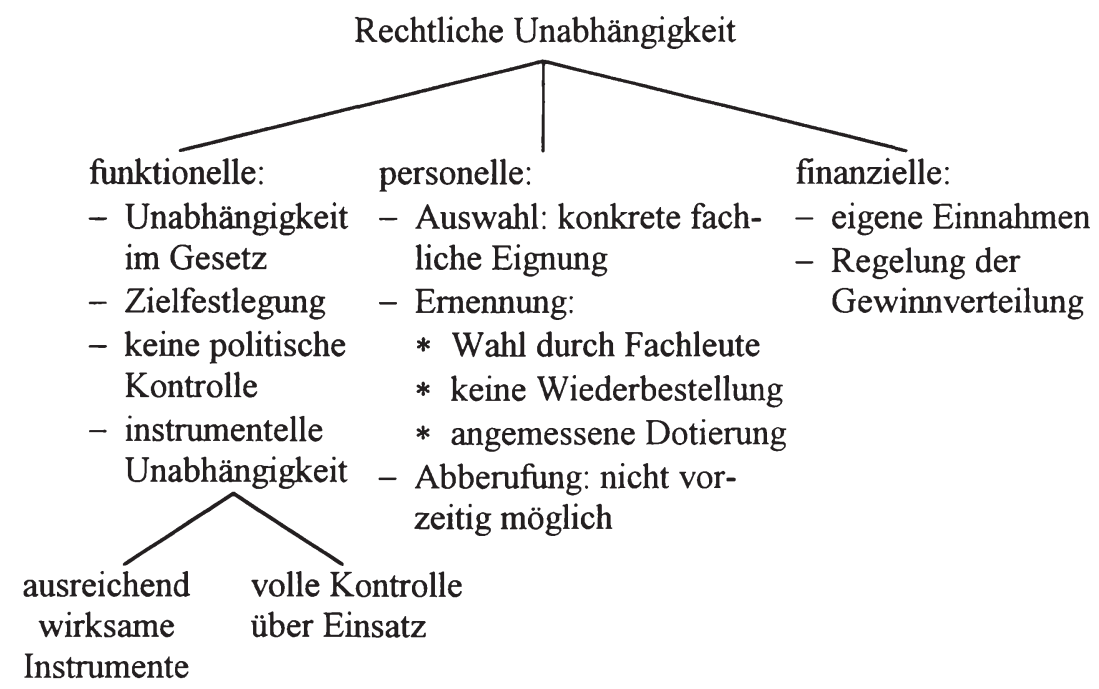

Die funktionelle Unabhängigkeit beinhaltet, daß die Unabhängigkeit eindeutig im Gesetz festgelegt und die Zentralbank klar auf das Ziel der Preisniveaustabilität verpflichtet wird. Damit sich die Zentralbank ihrer Unabhängigkeit sicher sein könnte und nicht eventuellen „Erpressungsversuchen“ der Regierung nachgeben müßte, da sie das Gesetz einfach aufheben könne, sollte der Zentralbankstatus Verfassungsrang erhalten oder zumindest die Hürde einer qualifizierten Mehrheit erfordern. Die klare Zielvorgabe im Gesetz bedeutet, daß die Zentralbank nicht zur Unterstützung anderer Politikbereiche herangezogen werden darf, da so eine Zielverletzung mit der Verfolgung eines anderen Ziels entschuldigt werden könnte. Insofern ist die eindeutige Zielvorgabe zwar eine Beschränkung der Unabhängigkeit ${ }^{38}$, allerdings eine der Reputation förderliche. Die explizite Zielvorgabe muß aber die einzige Beschränkung für die Entscheidungsmöglichkeit der Zentralbanker bleiben. Es darf der Regierung nicht gestattet sein, durch Weisungs-, Genehmigungs-, Veto- oder Kontrollrechte die Entscheidungen der Zentralbankratsmitglieder zu manipulieren. Selbst die Pflicht zur Koordination oder Kooperation mit der Regierung kann sich als Hemmnis für eine strikte Antiinflationspolitik erweisen und sollte aus diesem

In der Literatur findet man häufig die Forderung, die Zentralbank müsse ihre wirtschafts- und währungspolitischen Zielprioritäten selbst setzen dürfen, um vollständig unabhängig zu sein. Solch eine weitgehende Unabhängigkeit wird hier nicht gefordert, da sie zudem dem Ziel der Erlangung von Reputation zuwiderlaufen könnte. 
Grunde unterbleiben. Die eindeutige Zielvorgabe genügt zudem zur Vermeidung bzw. Minimierung von Konfliktsituationen. Wenn jeder Akteur von vornherein weiß, daß die Zentralbank sich ausschließlich an die Erfüllung ihrer Aufgabe hält, kann er dies in seinen Entscheidungen berücksichtigen. Falls keine eindeutige Zielvorgabe im Gesetz erwähnt bzw. die Zentralbank auf mehrere Ziele gleichzeitig verpflichtet wird, sollte zumindest eine verbindliche Regelung für Konfliktsituationen im Gesetz getroffen werden, damit die Unabhängigkeit nicht eingeschränkt wird.

Bei Verfolgung ihrer Aufgabe sollte die Zentralbank auch unabhängig vom nationalen Parlament sein, d.h. sie sollte keiner demokratischen Kontrolle unterliegen, die von Politikern wahrgenommen wird. Die Unabhängigkeit ist gerade als Schutz vor politischem Einfluß gedacht. Eine politische Kontrolle würde diesen Schutz wieder aufheben und die Unabhängigkeit konterkarieren. ${ }^{39}$

Damit die funktionelle Unabhängigkeit von praktischer Bedeutung ist, muß die Zentralbank nicht nur über die entsprechende Macht, sondern auch über die Möglichkeit verfügen, diese Unabhängigkeit umzusetzen. Hiermit ist die instrumentelle Ebene der Unabhängigkeit angesprochen. Die Zentralbank muß nicht nur über ein ausreichendes und wirksames Instrumentarium verfügen, sie sollte auch die volle Kontrolle darüber besitzen. Jeglicher Einfluß von seiten der Politik auf den Einsatz bzw. Nichteinsatz des Instrumentariums oder Verzögerung von Maßnahmen durch aufschiebendes Vetorecht begrenzen die Unabhängigkeit der Zentralbank. Ein wesentlicher Punkt ist auch das explizite Verbot der Kreditvergabe an die Regierung. Dies käme bei unbegrenztem Umfang einer Subordination der Geldpolitik unter die Fiskalpolitik gleich. Da die Inflationen der Vergangenheit meistens aber durch die Regierung verursacht wurden, sollte die Zentralbank weder direkte Kredite an die Regierung gewähren noch diese indirekt über den Kauf von Offenmarktpapieren am Primärmarkt finanzieren. Strikte Budgetrestriktionen für die Regierung können eine Fiskalpolitik, die nicht inflationstreibend wirkt, begünstigen.

Ein weiterer wichtiger Punkt der instrumentellen Unabhängigkeit ist die Entscheidungskompetenz für die Wechselkurspolitik. Nur wenn ausschließlich die Zentralbank über Wechselkursvereinbarungen bzw. -anpassungen entscheidet,

39 Dieser Punkt ist in der Literatur allerdings umstritten. Es werden teilweise parlamentarische Kontrollen oder Rechenschaftspflichten gegenüber dem Parlament gefordert, genau um die Geldwertstabilität zu erhöhen. Dies ist jedoch nicht der Fall, wie man anhand der Erfahrungen des Federal Reserve Systems (Fed) der USA sehen kann. Hier wird es deshalb als Reputationssteigerung gesehen, wenn Politiker überhaupt keinen Einfluß haben, auch nicht über die Kontrolle. 
kann davon ausgegangen werden, daß die binnenwirtschaftliche Geldpolitik nicht durch außenwirtschaftliche Einflüsse konterkariert wird. Bei geteilter Kompetenz für Geld- und Währungspolitik wird die formale Unabhängigkeit de facto aufgehoben, weil die Regierung mit Hilfe der Wechselkurspolitik den geldpolitischen Kurs der Zentralbank unterlaufen kann, wie dies in der Praxis immer wieder geschieht. Die Erfahrung spricht deshalb dafür, zur wirksamen Inflationsbekämpfung Geld- und Währungspolitik in eine Hand zu legen.

Die funktionelle Unabhängigkeit ist aber nur zu verwirklichen, wenn die Mitglieder der Zentralbank auch in personeller Hinsicht unabhängig sind. Hier sind insbesondere die Umstände der Auswahl, Ernennung und Abberufung der Zentralbankratsmitglieder angesprochen. Damit der politische Einfluß nicht durch „die Hintertür" in die Zentralbank dringt, muß eine parteipolitische Durchdringung des Zentralbankrats verhindert werden. Einen Beitrag könnte die Forderung nach hohen fachlichen Ansprüchen an die Mitglieder des Zentralbankrats leisten. Diese gewünschte Qualifikation sollte allerdings explizit im Gesetz konkretisiert sein. Eine Formel wie „eine besondere fachliche Eignung" ist dazu nicht geeignet, da sie erhebliche Interpretationsspielräume läßt.

Der immer wieder auftauchende Verweis auf den Beckett-Effekt ist als Argument für eine Inflationsaversion nicht stichhaltig. Es wird implizit unterstellt, daß die Mehrheit der Mitglieder des Zentralbankrats inflationsavers ist und die neu dazu kommenden „Politiker“ sich automatisch der Mehrheitsmeinung anschließen, d.h. auch Inflationsgegner werden, selbst wenn sie es vorher noch nicht waren. Wie aber wirkt der Beckett-Effekt, wenn die Mitglieder des Zentralbankrats keine Inflationsgegner sind? Dann könnte theoretisch ein Inflationsgegner zum Inflationsbefürworter werden. Es ist also zu berücksichtigen, $\mathrm{da} ß$ der Beckett-Effekt in unterschiedliche Richtungen wirken kann.

Bei der Auswahl muß deshalb besonderer Wert auf die fachliche Kompetenz gelegt werden. Dies wäre um so eher gegeben, als nicht Politiker die Mitglieder des Zentralbankrats wählen dürfen, sondern Fachleute. Dies würde auch ein eventuelles „Wohlverhalten“ der Zentralbanker zwecks Wiederbestellung verhindern. Besser als eine Wiederbestellung wäre ohnehin eine lange Amtszeit ohne die Möglichkeit der Wiederbestellung, aber auch ohne vorherige willkürliche Abrufbarkeit. Der Vorschlag einer flexiblen Gestaltung der Amtszeit in Abhängigkeit des Alters insofern, als das Mitglied des Zentralbankrats nach seiner Zeit in der Zentralbank das aktive Berufsleben beenden sollte, damit es nicht sein Amt im Hinblick auf spätere Anstellungschancen ausnutzt, kann nicht als Lösung des Problems der persönlichen Abhängigkeit betrachtet werden. Das Erreichen des Pensionsalters ist in der Praxis kein Hindernis, auch 
weiterhin in der Politik oder in einer Beraterfunktion tätig zu sein. Ein „Wohlverhalten" von seiten der Zentralbanker gegenüber Politikern ist also nicht vollkommen auszuschließen. Eine angemessene Dotierung, bei der die Privatwirtschaft als Richtschnur dient, könnte schließlich gewährleisten, daß einerseits auch tatsächlich die besten Fachkräfte kommen und andererseits dieser Posten nicht lediglich als Sprungbrett in die private Wirtschaft betrachtet wird. Wären diese Voraussetzungen erfüllt, könnte weitestgehend von einer personellen Unabhängigkeit gesprochen werden.

Neben der funktionellen und personellen Unabhängigkeit muß noch der finanzielle Aspekt berücksichtigt werden, damit die Zentralbank unabhängig die Inflation bekämpfen kann. Die finanzielle Unabhängigkeit bedeutet, daß die Zentralbank über eigene Einnahmen verfügt, einen eigenen Haushalt hat und selbständig bilanziert. Eine Haushaltskontrolle darf nicht von Politikern, sondern nur von unabhängigen Wirtschaftsprüfern vorgenommen werden. Zudem muß eine eindeutige Regelung zur Gewinnverteilung vorgesehen sein bei gleichzeitiger Gewähr, daß der oder die Begünstigten keinen politischen Druck auf die Zentralbank ausüben können, um die Höhe des Gewinns zu steigern.

Auch wenn alle drei Bereiche der Unabhängigkeit erfüllt sind, ist noch nicht gewährleistet, daß diese rechtliche Autonomie tatsächlich durchsetzbar ist. Die juristische Unabhängigkeit ist nutzlos, falls die Zentralbank faktischen Handlungszwängen in Form politischer Pressionen oder ökonomischer Restriktionen unterliegt. Die rechtliche Unabhängigkeit kann bei faktischer Abhängigkeit sogar als raffinierter Schachzug der Regierung betrachtet werden, sich für schlechte wirtschaftliche Daten einen Sündenbock besorgt zu haben, während sie alle guten Ergebnisse für sich verbucht. ${ }^{40}$

\subsubsection{Tatsächliche Unabhängigkeit}

Die rechtliche Unabhängigkeit liefert nur den juristischen Rahmen für die tatsächliche Unabhängigkeit. Je nachdem, wie konkret die gesetzlichen Bestimmungen sind, kann die politische und ökonomische Realität die tatsächliche Unabhängigkeit der Zentralbank relativieren. Diese faktische Unabhängigkeit der Zentralbank hängt wesentlich von ihrer informellen Macht $a b$, über die letztlich die Öffentlichkeit befindet. Der Gesetzgeber andererseits entscheidet darüber, welche Rolle die informelle Macht spielen muß. Je höher die infor-

40 Vgl. Berthold (1992), S. 28, Dittus (1987), S. 183, Filc (1994), S.700f., Gischer (1993), S. 62, Hasse (1989a), S. 115-120, Loef (1990), S. 107-109, Neumann (1990), S. 67-69, Socher (1991), S. 388 und Woll (1988), S. 122-124. Zur „Sündenbock-Funktion“ vgl. Kane (1980), der dies für die Fed festgestellt hat. 
melle Macht ist, desto mehr können sowohl die politischen Pressionen, die dazu führen, daß geldpolitische Entscheidungen erst gar nicht getroffen werden, als auch die ökonomischen Restriktionen minimiert werden. Letztere liegen vor, wenn Entscheidungen zwar getroffen werden, aufgrund konterkarierender Verhaltensweisen anderer Wirtschaftsgruppen oder eines nicht effizienten Instrumentariums, nicht wirken und somit den Aufbau von Reputation über geringe Inflationsraten verhindern.

Ob eine Zentralbank politischen Zwängen unterliegt, ist insbesondere vom Konfliktpotential der Geldpolitik sowie von der Konfliktbereitschaft der beteiligten Akteure abhängig, weshalb in der Literatur oft die Häufigkeit sowie der Ausgang von Konflikten als Hilfskriterien für den tatsächlichen politischen Handlungsspielraum verwendet werden. Das Konfliktpotential ist um so geringer, je präziser die Angaben zur Zielsetzung und Stellung der Notenbank gegenüber der Regierung im Gesetz sind, je weniger Interpretationsspielräume das Gesetz also läßt. Auch in Zeiten einer Rezession, in der das Konfliktpotential der Geldpolitik tendenziell höher ist, darf kein Zweifel daran bestehen, daß die Zentralbank primär die Geldwertstabilität zu sichern hat. Das Gesetz sollte der Regierung kein Drohpotential gegenüber der Zentralbank belassen insofern, als die Regierung bei nicht genehmen Entscheidungen einer unabhängigen Zentralbank deren Status durch eine Gesetzesänderung einfach aufhebt.

Des weiteren sind aber auch alle anderen Forderungen, wie sie im letzten Abschnitt gestellt wurden, zu erfüllen, damit das Konfliktpotential möglichst minimiert wird. Insbesondere ist noch einmal das Währungssystem herauszuheben, da das Konfliktpotential der Geldpolitik mit dem Ausland um so größer ist, je fester die Wechselkurse aneinander gebunden sind und demzufolge die Pflicht der Zentralbank zur Intervention besteht. Diese stellt gleichzeitig eine ökonomische Restriktion der Zentralbank dar, weil es ihr trotz antiinflationärer heimischer Geldpolitik nicht gelänge, sich vom internationalen Inflationsniveau abzukoppeln.

Aber auch die Zentralbank selbst hat die Möglichkeit, das Konfliktpotential zu mindern, indem sie sich monetäre Zwischenziele vorgibt, die sie öffentlich verkündet. Diese Selbstbindung stellt einen Schutz vor politischen Pressionen dar und kann offene Konflikte vermeiden. Ob es zu offen ausgetragenen Konflikten kommt, ist abhängig von der Konfliktbereitschaft der beteiligten Akteure. Hierbei kann auch das „Element des Persönlichen“ zum Tragen kommen, wonach 
eine starke Persönlichkeit an der Zentralbankspitze erheblich zur Reputationssteigerung beitragen kann. ${ }^{41}$

\subsubsection{Die Zentralbank als Eigennutzmaximierer}

Selbst wenn eine Zentralbank ein Höchstmaß an rechtlicher und faktischer Unabhängigkeit genießt, ist nicht gesichert, daß sie primär versucht, über geringe Inflationsraten ihre Reputation zu maximieren. ${ }^{42}$ Es ist theoretisch inkonsistent, aufgrund der Erkenntnisse der Neuen Politischen Ökonomie für alle Akteure inklusive der Politiker eigennutzorientiertes Handeln anzunehmen, den Zentralbankern aber altruistische Motive zu unterstellen. Diese Sichtweise entspräche einer sehr weiten Interpretation der Weberschen Bürokratietheorie, wonach Fachleute ihre Aufgaben nach bestem Wissen und Gewissen erfüllen. Angebrachter scheint die Sichtweise der Neuen Institutionenökonomie zu sein, nach der jede Institution primär ihre eigenen Interessen verfolgt. ${ }^{43}$ Dann muß aber zuerst geklärt werden, welche Interessen eine Zentralbank überhaupt verfolgen könnte, d.h. welche Ziele sie anstrebt, um ihren Nutzen zu maximieren.

In der Literatur werden verschiedene Arten von Nutzenfunktionen für Zentralbanken diskutiert. Es wird dabei davon ausgegangen, daß alle Mitglieder des Zentralbankrats die gleichen Präferenzen haben, da ein Außenstehender keinen Einblick in die internen Auseinandersetzungen des Zentralbankrats hat. Deshalb werden zunächst die Interessen der Zentralbankratsmitglieder mit denen der Zentralbank gleichgesetzt.

Im wesentlichen findet man drei Arten von Zielfunktionen für die Zentralbank in der Literatur:

a. Prestige-Sicherheits-Ansätze,

b. Optimierung des Handlungsspielraums oder der Macht,

c. Maximierung der diskretionären Ausgaben.

41 Zur ausführlichen Diskussion der politischen und ökonomischen Restriktionen einer Zentralbank vgl. Caesar (1981).

42 Auch auf das Problem der X-Ineffizienz der Zentralbank muß hingewiesen werden, wenn sie als nicht kontrollierbare Bürokratie gilt. Da es aber noch keine empirischen Studien über das Verhältnis zwischen Unabhängigkeit und X-Ineffizienz gibt, soll dieser Punkt hier nicht weiter verfolgt werden. Vgl. auch Neumann (1992), S. 73.

43 Die ersten Arbeiten, die sich explizit mit dem Verhalten von Zentralbanken beschäftigen, verwenden den Rahmen der Bürokratietheorie. Vgl. Acheson/Chant (1972), (1973a) und (1973b). Da dieser Rahmen jedoch zu eng erscheint und zudem für die Zentralbank nicht immer ganz passend ist, wird hier der weitere Rahmen der Neuen Institutionenökonomie verwendet. Vgl. dazu Issing (1982), S. 58f. und Puchta (1981), S. 185-190. 
Das Nutzenelement Einkommen, welches für Bürokraten auch immer wieder genannt wird - und nichts anderes sind die Zentralbanker im weitesten Sinne kann als implizit enthalten betrachtet werden. Unterscheidet man zwischen erfolgsunabhängigem und erfolgsabhängigem Einkommen, ist ersteres über das Element Sicherheit abgedeckt. Nur im Falle des Verbleibens im Amt, was der Faktor Sicherheit zum Ausdruck bringt, ist von einer Fortzahlung eines sicheren Gehaltes auszugehen. Die erfolgsabhängige Entlohnung kann durch das Element Prestige abgedeckt werden, da dies auch den Erfolg einer Zentralbank widerspiegelt.

Der Begriff „Prestige“ wird im Rahmen dieser Arbeit jedoch differenzierter betrachtet ${ }^{44}$ Hier wird nicht unterstellt, daß die Bevölkerung immer inflationsavers ist und primär eine antiinflationäre Zentralbankpolitik wünscht. Reputation und Prestige wären sonst synonym zu verwenden und stellten ein einziges Ziel dar. Sobald diese Annahme aber aufgegeben wird, können zwei unterschiedliche Ziele vorliegen. Prestige wird dann interpretiert als Ansehen bzw. Beliebtheit bei der Bevölkerung, was durch opportunistisches Verhalten erreicht wird. Reputation hingegen wird immer nur durch eine Antiinflationspolitik erreicht. Man kann in diesem Sinne auch von Respekt bzw. Hochachtung für eine erbrachte Leistung sprechen. Die Zentralbank kann also bei einer inflationsaversen Bevölkerung nur durch eine strikte Antiinflationspolitik sowohl Reputation als auch Prestige erlangen. Bei einer weniger inflationsaversen Bevölkerung kann sie Reputation ebenfalls nur durch eine Antiinflationspolitik erlangen, dies schadet jedoch ihrem Prestige. Letzteres kann sie nur durch Vernachlässigung der Preisstabilität zugunsten anderer von der Bevölkerung präferierter Ziele erlangen, was dann aber zum Verlust der Reputation führt. Bei einer weniger inflationsaversen Bevölkerung muß die Zentralbank sich also entscheiden, ob sie Reputation oder Prestige erlangen will.

Als viertes mögliches Ziel einer Zentralbank wird in der Literatur die Maximierung des Handlungsspielraums genannt. Der Handlungsspielraum eigennutzorientierter Zentralbanken wird als Freiraum für eigenständiges und eigenzielorientiertes Handeln verstanden. ${ }^{45}$ Dies ist durch das allgemeinere Ziel Machterlangung abgedeckt. Da das letztere als wesentliches Ziel in der Bürokratietheorie akzeptiert ist, wird es auch hier berücksichtigt. Zudem ist es umfassender als das Ziel der Handlungsspielraummaximierung. Macht wird hier im

Vgl. hierzu auch Kapitel 2.2.

45 Vgl. Puchta (1981), S. 226. Anders definiert Hansmeyer den Handlungsspielraum für eine gemeinwohlorientierte Zentralbank als den Freiraum, welcher nicht durch juristische, ökonomische oder politische Faktoren eingeschränkt wird. Vgl. Hansmeyer (1968) S. 155ff. 
Sinne Webers als Möglichkeit zur Durchsetzung des eigenen Willens auch gegen Widerstand verstanden. Externe Widerstände sind aber innerhalb des Handlungsspielraums der Zentralbank nicht zu erwarten.

Einige Ansätze berücksichtigen das Ziel der Maximierung der diskretionären Ausgaben für eine Zentralbank. Dieser Ansatz entspricht dem der Budgetmaximierung ${ }^{46}$ für die Bürokratietheorie. $\mathrm{Zu}$ den diskretionären Ausgaben sind die Ausgaben zu zählen, die den Zentralbankleitern direkt zugute kommen, wie etwa Dienstreisen, gut ausgestattete Büros und ähnliches mehr. Die primäre Verfolgung dieses Ziels wird hier aber als unrealistisch eingeschätzt und nicht weiter berücksichtigt, da das gleiche oder sogar noch bessere Ergebnisse in anderen Behörden bei weitaus geringerem Arbeitseinsatz zu erreichen sind.

Zusammenfassend bleibt festzuhalten, daß mögliche „Oberziele“ eigennutzorientierter Zentralbanker die Erlangung von Prestige, Reputation oder Macht, die Erzielung von Einkommen oder einfach die Erhaltung der Sicherheit sein können. Welches dieser sich teilweise widersprechender Ziele primär von einem Zentralbanker angestrebt wird, hängt von dessen subjektiver Einstellung ab.

Um konkrete Handlungen der Zentralbank erklären zu können, müssen Unterziele zur Konkretisierung des allgemeinen primären Ziels formuliert werden. Genau hier liegt aber das Problem, da die konkrete Ausgestaltung einer Nutzenfunktion von den Restriktionen des polit-ökonomischen Gesamtsystems abhängig ist. Die zu beachtenden Bedingungen zur Formulierung der Zielfunktion und der Nebenbedingungen variieren von Land zu Land und können auch innerhalb eines Landes zu verschiedenen Zeitpunkten unterschiedlich sein. Ein wesentlicher Einflußfaktor sind zudem die historischen Erfahrungen. Welche Unterziele eine Zentralbank im konkreten Fall verfolgt, ist nicht nur von ihrem primären Oberziel abhängig, sondern auch vom jeweiligen Aktionsrahmen. Eine konkrete Nutzenfunktion der Zentralbank ist deshalb immer nur für den Einzelfall zu beschreiben. ${ }^{47}$

Neben der Nutzenfunktion der Zentralbank spielt die Präferenzstruktur der Bevölkerung eine wesentliche Rolle für die Reputation. Deshalb muß auch für die privaten Wirtschaftssubjekte eine Nutzenfunktion aufgestellt werden. Hier gilt allerdings die gleiche Restriktion wie für die Zentralbank, daß dies nur für den konkreten Einzelfall erfolgen kann. Da in der Literatur aber meist unterstellt

46 Vgl. Niskanen (1975).

47 Vgl. Kösters (1990), S. 129ff., Puchta (1981), S. 154 und 176ff. und Willeke (1993), S. 29ff. und 68ff. Einen Überblick über in der Literatur verwendete Nutzenfunktionen für Zentralbanken gibt Willeke (1993), S. 74f. 
wird, daß die Bevölkerung von der Zentralbank Geldwertstabilität nachfragt, wird dieser Punkt hier aufgegriffen und relativiert. In dieser Arbeit wird gerade davon ausgegangen, daß die Bevölkerung nicht immer Geldwertstabilität nachfragt. $\mathrm{Zu}$ beachten sind hier die Inflationsmentalität der Bevölkerung sowie die Tatsache, daß das von der Zentralbank angebotene Gut Geldwertstabilität ein öffentliches Gut darstellt. Selbst wenn sich eine große latente Gruppe aufgrund ihrer Vorliebe für Stabilität dieses Gut wünschen sollte, wird sie ihr Interesse kaum als Organisation vorbringen. ${ }^{48}$ Fehlt das Interesse an diesem Gut eventuell ganz, weil sein Wert unterschätzt wird, kann die Geldwertstabilität sogar als meritorisches Gut betrachtet werden, da es dann trotz fehlenden individuellen Bedürfnisses im öffentlichen Interesse liegt, daß dieses Gut angeboten wird. Ist dieser Fall gegeben, muß das Angebot „Zwangsweise“ erfolgen. ${ }^{49}$ Wenn dann eine Zentralbank die Geldpolitik bestimmt, deren primäres Interesse z.B. in der Erzielung von Prestige liegt, wird sie dieses gesellschaftlich wünschenswerte Gut freiwillig nicht anbieten. Hier wäre dann die Situation gegeben, daß auch eine unabhängige Zentralbank die Preisniveaustabilität nicht garantieren würde.

\subsubsection{Die Bedeutung der Unabhängigkeit der Zentralbank für das Modell}

Die Reputation einer Zentralbank ist vom institutionellen Rahmen abhängig. Entsprechend Gleichung (2.19) werden die Inflationserwartungen in Abhängigkeit der Machtverhältnisse gesetzt. Bei zunehmender Unabhängigkeit der Zentralbank werden die Wirtschaftssubjekte ihre Erwartungen mindern, was zu einer geringeren optimalen Inflationsrate für die Zentralbank führt. Die Realisation einer geringeren Inflationsrate wiederum führt zur Reputationssteigerung der Zentralbank.

(2.19) $\downarrow \Pi^{\mathrm{e}}=\uparrow \gamma \cdot \Pi_{\mathrm{ZB}}^{\mathrm{e}}+\downarrow(1-\gamma) \cdot \Pi_{\mathrm{P}}^{\mathrm{e}}$ wegen: $\Pi_{\mathrm{ZB}}^{\mathrm{e}}<\Pi_{\mathrm{P}}^{\mathrm{e}}$

Die gesunkene Erwartung beeinflußt die optimale Inflationsrate $\Pi^{\mathrm{ZB}}$ :

$$
\downarrow \Pi^{\mathrm{ZB}}=\frac{\mathrm{b}}{\mathrm{a}} \cdot \sqrt{\frac{2 \cdot \mathrm{a}}{\mathrm{b} \cdot(1+\mathrm{q})} \cdot \downarrow \Pi^{\mathrm{e}}-\frac{1-\mathrm{q}}{1+\mathrm{q}}}
$$

Zum Problem der Organisierbarkeit großer Gruppen siehe Olson (1968), insbesondere S. $163 \mathrm{f}$.

49 Vgl. Kirsch (1982), S. 34ff. Zur Theorie öffentlicher Güter im allgemeinen und meritorischer Güter im besonderen vgl. Zimmermann/Henke (1987), S. 39ff. 
Die Minderung der optimalen Inflationsrate der Zentralbank führt nun ihrerseits über Gleichung (2.17) dazu, daß die tatsächliche Inflationsrate sinken kann:

(2.17) $\Pi=\Pi_{\mathrm{GP}}+\Pi_{\mathrm{FP}}+\Pi_{\mathrm{R}}$

Durch Einsetzen der Gleichung (2.18) und Zusammenfassen der beiden von der Zentralbank nicht zu kontrollierenden Teilinflationsraten, deren Höhe mit $2 \%$ angenommen wurde, ergibt sich:

$$
\downarrow \Pi=\uparrow \gamma \cdot \downarrow \Pi_{Z B}+\downarrow(1-\gamma) \cdot \Pi_{P}+2 \% \text { wegen: } \Pi_{Z B}^{\mathrm{e}}<\Pi_{\mathrm{P}}^{\mathrm{e}}
$$

Die tatsächlich realisierte Inflationsrate wird gleich in zweifacher Hinsicht von der zunehmenden Unabhängigkeit der Zentralbank getroffen. Die gestiegene Unabhängigkeit der Zentralbank kommt durch ein steigendes $\gamma$ zum Ausdruck, der Einfluß der Zentralbank auf die realisierte Inflationsrate steigt also. Die Zentralbank kann die Inflationsrate nahezu perfekt kontrollieren, wenn alle „Unabhängigkeitskriterien“, wie sie oben beschrieben wurden, erfüllt sind. Sie bestimmt dann nicht nur allein die Geldpolitik, sondern begrenzt in gewissem Umfang auch den Einfluß der Fiskalpolitik auf die Inflationsrate. Der Wert von $\gamma$ liegt dann nahe Eins. Hinzu kommt, daß die gestiegene Reputation, die sich in den Erwartungen der Wirtschaftssubjekte widerspiegelt, eine geringere von der Zentralbank zu verantwortende Teilinflationsrate $\left(\Pi_{\mathrm{ZB}}\right)$ zuläßt. Der positive Gesamteffekt auf die tatsächliche Rate wird also verstärkt.

Wenn diese Argumentationskette gilt, was zunächst sinnvoll erscheint, da die Regierung mehreren Anreizen zur Inflationierung unterliegt ${ }^{50}$, ist es nur folgerichtig, eine möglichst umfassende Unabhängigkeit für die Zentralbank zu fordern, damit $\gamma$ steigt. Gleichzeitig würde auch die Gewichtung für das Stabilitätsziel a steigen bzw. für das Beschäftigungsziel b sinken, da annahmegemäß eine Zentralbank um so stabilitätsbewußter sein kann, je unabhängiger sie ist. Das gestiegene a würde auch zu einem steigenden q führen, d.h. die Bedeutung der Zukunft würde wichtiger. Eine größere Berücksichtigung der Zukunft bedeutet aber nichts anderes als eine höhere Bewertung der Kosten aus einer Überraschungsinflation, und somit würde im Modell bei gleichbleibendem Anreiz die gleichgewichtige Inflationsrate sinken. Bei verändertem Stabilitätsbewußtsein sinkt der Anreiz zur Inflation aber sogar noch, und so würde auch die Nutzenbewertung der Inflation in die gleiche Richtung wirken. Damit diese positiven Effekte auf die Reputation und damit letztlich auf die Inflationsrate wirken können, müssen aber nicht nur alle Voraussetzungen, wie sie im Kapi-

Vgl. dazu Kapitel 3.1.2. 
tel 3.3.2 beschrieben wurden, strikt erfüllt sein, sondern neben diesen rechtlichen Voraussetzungen ist auch die faktische Unabhängigkeit zu gewährleisten. Es muß sichergestellt werden, daß die politischen Pressionen und ökonomischen Restriktionen minimiert werden. Wie gezeigt wurde, ist dies am ehesten durch präzise Angaben im Gesetz zu erzielen. In erster Linie darf es keine Interpretationsspielräume bezüglich des von der Zentralbank zu verfolgenden Ziels Preisniveaustabilität zulassen, noch darf die gesetzlich verankerte Unabhängigkeit zum „Spielball“ der Politiker werden.

Geht man jedoch realistischerweise davon aus, daß die Zentralbank genau wie alle anderen Akteure ihren Eigennutz maximieren will, sind die Effekte auf die Reputation nicht so eindeutig wie oben beschrieben. Das Verhalten der Zentralbank wird dann von ihrer individuellen Nutzenfunktion und den zugrundeliegenden Rahmenbedingungen bestimmt. Um nun die Effekte auf die Reputation zu zeigen, werden nur die Extremfälle einer völlig unabhängigen $(\gamma=1)$ und einer völlig abhängigen $(\gamma=0)$ Zentralbank mit vollständiger Inflationsaversion $(a=1)$ oder vollständig fehlendem Stabilitätsbewußtsein $(a=0)$ unterstellt:

Für den Fall, daß die Zentralbank in erster Linie versucht, Prestige zu erzielen, ist die Frage nach der Einstellung der Bevölkerung zu beantworten. Da Prestige hier im Sinne von Beliebtheit bei der Bevölkerung verstanden wird, muß die Zentralbank dann eine Geldpolitik verfolgen, die die Bevölkerung sich wünscht. Tabelle 3.4 enthält die acht denkbaren Fälle.

Die Zentralbank wird unabhängig von ihrem Status und Stabilitätsbewußtsein immer die Inflationsrate realisieren, die den Wünschen der Bevölkerung entspricht. Unabhängig von ihren eigenem Stabilitätsbewußtsein wird sie zur Bestimmung ,ihrer" optimalen Inflationsrate a immer entsprechend den Wünschen der Bevölkerung gewichten. Nur für den Fall einer inflationsaversen Bevölkerung kann die Zentralbank über den Aufbau von Reputation auch Prestige erzielen. Gilt die Zentralbank durch die Wahl eines hohen a als Inflationsgegner, ist sie bei einer stabilitätsbewußten Bevölkerung angesehen (Fälle 1, 3, 5 und 7). Die Inflationsrate wird sich dann gegen Null bewegen. Eine Antiinflationspolitik ist glaubwürdiger, die Wirtschaftssubjekte werden zukünftig geringere Inflationsraten auch erwarten, und der Gesellschaft entstehen durch die Wahl geringer Inflationsraten keine Anpassungskosten. Gewichtet die Bevölkerung jedoch andere Ziele mehr und macht die Zentralbank für deren Realisation mitverantwortlich (Fälle 2, 4, 6 und 8), gelingt es der Zentralbank nur dann, Ansehen zu erlangen, wenn sie auch das andere Ziel berücksichtigt (b also einen positiven Nutzen beimißt) und dafür auf eine konsequente Inflationsbekämpfung $(\mathrm{a}=1)$ verzichtet. In diesen Fällen bewertet die Bevölkerung 
Tabelle 3.4: Prestige, Stabilitätsbewußtsein und Inflation

\begin{tabular}{|c|c|c|c|c|}
\hline \multirow[t]{2}{*}{$\mathrm{Nr}$. } & \multirow[t]{2}{*}{ Unabhängigkeit } & \multicolumn{2}{|c|}{ Stabilitätsbewußtsein } & \multirow[t]{2}{*}{ Inflation $\Pi$} \\
\hline & & der Zentralbank & der Bevölkerung & \\
\hline 1 & $\gamma=1$ & $a=1$ & inflationsavers & $\Pi \rightarrow 0$ \\
\hline 2 & $\gamma=1$ & $a=1$ & $\begin{array}{c}\text { nicht } \\
\text { inflationsavers }\end{array}$ & $\overline{\Pi \uparrow}$ \\
\hline 3 & $\gamma=1$ & $a=0$ & inflationsavers & $\Pi \rightarrow 0$ \\
\hline 4 & $\gamma=1$ & $a=0$ & $\begin{array}{c}\text { nicht } \\
\text { inflationsavers }\end{array}$ & $\Pi \uparrow$ \\
\hline 5 & $\gamma=0$ & $a=1$ & inflationsavers & $\Pi \rightarrow 0$ \\
\hline 6 & $\gamma=0$ & $a=1$ & $\begin{array}{c}\text { nicht } \\
\text { inflationsavers }\end{array}$ & $\Pi \uparrow$ \\
\hline 7 & $\gamma=0$ & $a=0$ & inflationsavers & $\Pi \rightarrow 0$ \\
\hline 8 & $\gamma=0$ & $\mathrm{a}=0$ & $\begin{array}{c}\text { nicht } \\
\text { inflationsavers }\end{array}$ & $\Pi \uparrow$ \\
\hline
\end{tabular}

die Reputation eher negativ, weil sie die Gefahren der Inflation nicht erkennt und somit auch nicht den positiven Charakter der Reputation. Die Zentralbank wird dann aus Prestigegründen tendenziell auf den Aufbau von Reputation verzichten und zur Erreichung anderer Ziele positive Inflationsraten zulassen. Um mit der Geldpolitik aber reale Effekte zu erzielen, müssen die positiven Inflationsraten für die Wirtschaftssubjekte überraschend kommen, d.h. sie dürfen deren korrekte Höhe nicht erwartet haben. Für die Glaubwürdigkeit bedeutet dies, daß die Zentralbank ihre Ankündigung nicht einhalten darf, selbst wenn sie erwartet war. Langfristig werden die Wirtschaftssubjekte der Ankündigung einer Antiinflationspolitik aber keinen Glauben mehr schenken, und die Geldpolitik wird unglaubwürdig. Die von der Zentralbank realisierte Inflationsrate muß von Periode zu Periode höher ausfallen, um überraschend hoch zu sein.

Versucht die Zentralbank hingegen in erster Linie, Respekt (also Reputation) zu erlangen, wird sie unabhängig von ihrem Status immer die Inflation bekämpfen wollen, also a immer mit (fast) Eins gewichten. Die von der Zentralbank individuell gewünschte Inflationsrate von Null stimmt mit der, die im Interesse der Bevölkerung liegt, überein (Fall 9). Im Falle dieser Interessenharmonie stellt die Inflation kein Problem dar, weil die Erlangung von Reputation auch das individuelle Ziel der Zentralbank ist. Glauben die Wirtschaftssubjekte al- 
lerdings zunächst nicht daran, daß die Zentralbank primär ihre Reputation steigern will, werden sie eine angekündigte Antiinflationspolitik für nicht glaubwürdig halten. Die Zentralbank wird dann eine überraschend geringe Inflationsrate realisieren müssen, um die Erwartungen zu „brechen“, und so Arbeitslosigkeit produzieren. Diese ist dann als Preis für die Reputation zu sehen. Langfristig werden die Wirtschaftssubjekte der Ankündigung einer Antiinflationspolitik aber glauben, und eine Stabilisierungskrise aus Gründen des Reputationsaufbaus ist nicht mehr erforderlich.

Sollte die Zentralbank jedoch von der Regierung abhängig sein, reicht allein ihr Wille zum Aufbau von Reputation nicht aus. Trotz des primären Ziels der Zentralbank sich Reputation aufzubauen, kann dies dann nur bei einer stabilitätsbewußten Bevölkerung realisiert werden, da die Regierung die Macht über die Geldpolitik besitzt und diese die Wählerwünsche berücksichtigen wird (Fall 10).

Verfolgt die Zentralbank primär das Ziel der Machtsteigerung, wird die Inflationsrate im Falle der gesicherten Unabhängigkeit vom Stabilitätsbewußtsein der Zentralbank bestimmt, während wieder das Stabilitätsbewußtsein der Bevölkerung ausschlaggebend ist, wenn die Zentralbank abhängig ist. Die Gewichtung von a entspricht im ersten Fall den Präferenzen der Zentralbank, während im zweiten Fall die Bevölkerung darüber „bestimmt“. Im Falle der Unabhängigkeit besitzt sie bereits eine hohe Macht und kann diese ihren Interessen entsprechend nutzen. Ist die Zentralbank aber abhängig, ihre Macht demnach gering, wird sie versuchen, ihre Macht zu steigern, indem sie sich Rückhalt bei der Bevölkerung verschafft, sich also informelle Macht aufbaut. Hier wird sie wieder versuchen, Prestige zu erlangen, diesmal jedoch im Sinne eines Mittels zum Zweck. Durch eine positive Bewertung bei der Bevölkerung kann selbst eine rechtlich abhängige Zentralbank faktisch nahezu unabhängig sein. Durch die Erlangung von Prestige könnte eine abhängige Zentralbank über Macht verfügen, die ihr vom Gesetz so nicht verliehen war. Wieder sind acht Fälle zu unterscheiden.

Die Fälle 11 bis 14 in Tabelle 3.5 zeigen, daß für den Fall einer völlig unabhängigen Zentralbank, die sich ihrer Unabhängigkeit sicher sein kann, ausschließlich das eigene Stabilitätsbewußtsein ausschlaggebend ist für die gewählte Inflationsrate. Die Zentralbank wird a entsprechend ihren tatsächlichen Präferenzen wählen. Sieht sie ihre Sicherheit hingegen nicht gewährleistet, wird sie unabhängig vom eigenen Stabilitätsbewußtsein immer eine Inflationsrate wählen, die dem Stabilitätsbewußtsein der Bevölkerung entspricht. Da sie 
Tabelle 3.5: Macht, Stabilitätsbewußtsein und Inflation

\begin{tabular}{|c|c|c|c|c|}
\hline \multirow[t]{2}{*}{$\mathrm{Nr}$. } & \multirow[t]{2}{*}{ Unabhängigkeit } & \multicolumn{2}{|c|}{ Stabilitätsbewußtsein } & \multirow[t]{2}{*}{ Inflation $\Pi$} \\
\hline & & der Zentralbank & der Bevölkerung & \\
\hline 11 & $\gamma=1$ & $a=1$ & inflationsavers & $\Pi \rightarrow 0$ \\
\hline 12 & $\gamma=1$ & $a=1$ & $\begin{array}{c}\text { nicht } \\
\text { inflationsavers }\end{array}$ & $\Pi \rightarrow 0$ \\
\hline 13 & $\gamma=1$ & $a=0$ & inflationsavers & $\Pi \uparrow$ \\
\hline 14 & $\gamma=1$ & $a=0$ & $\begin{array}{c}\text { nicht } \\
\text { inflationsavers }\end{array}$ & $\Pi \uparrow$ \\
\hline 15 & $\gamma=0$ & $a=1$ & inflationsavers & $\Pi \rightarrow 0$ \\
\hline 16 & $\gamma=0$ & $a=1$ & $\begin{array}{c}\text { nicht } \\
\text { inflationsavers }\end{array}$ & $\Pi \uparrow$ \\
\hline 17 & $\gamma=0$ & $a=0$ & inflationsavers & $\Pi \rightarrow 0$ \\
\hline 18 & $\gamma=0$ & $a=0$ & $\begin{array}{c}\text { nicht } \\
\text { inflationsavers }\end{array}$ & $\Pi \uparrow$ \\
\hline
\end{tabular}

bei gefährdeter Sicherheit ihr Prestige steigern will, muß sie den Wünschen der Bevölkerung entsprechen (Fälle 15-18).

Im Fall 15 sind sowohl die Zentralbanker als auch die Bevölkerung inflationsavers, d.h. die Zentralbank maximiert hier ihr Prestige, indem sie ihren eigenen Präferenzen nachkommt. In diesem Fall können die Inflationserwartungen schon allein dadurch sinken, daß die Zentralbank unabhängig wird. Der sechzehnte Fall ist durch eine stabilitätsbewußte abhängige Zentralbank gekennzeichnet, die ihre Antiinflationspolitik nicht lange durchhalten kann, selbst wenn sie es wollte, da die Bevölkerung nicht inflationsavers ist. In diesem Fall kann die Zentralbank nur Prestige erlangen, wenn sie auf die Erzielung von Reputation verzichtet und a in Gleichung (2.25) tendenziell gering gewichtet.

Es kann demnach durchaus sein, daß es aufgrund ihres primären Ziels nicht im Interesse der Zentralbank liegt, Reputation zu erhalten (Fall 16). Andererseits kann eine Zentralbank auch „gezwungen“ werden, Reputation aufzubauen, obwohl sie dies eigentlich nicht will (Fall 17). Im letzten Fall (Fall 18) wollen weder die Zentralbank noch die Bevölkerung, daß erstere sich eine hohe Reputation aufbaut. Die Inflationsrate wird sich von Null weg bewegen. Ob die Zentralbank mit dieser Politik aber ihr primäres Ziel, Steigerung der Macht, erreichen kann, muß bezweifelt werden. Da die Zentralbank dafür letztlich Unab- 
hängigkeit benötigt, ist es sehr zweifelhaft, daß sie dies über öffentlichen Druck auf die Politiker erreichen kann, da die Bevölkerung überhaupt keine unabhängige Zentralbank wünscht.

Diese Fallunterscheidung sollte demonstrieren, daß weder die Unabhängigkeit noch ein hohes Stabilitätsbewußtsein der Bevölkerung hinreichend sind, um geringe Inflationsraten zu gewährleisten. Eine stabilitätsbewußte Bevölkerung wird aber in jüngster Zeit als notwendige und auch hinreichende Bedingung für eine an der Geldwertstabilität orientierte Geldpolitik gesehen. ${ }^{51}$ Die Unabhängigkeit sei notwendig, aber nicht hinreichend. Ob die Zentralbank Preisniveaustabilität anstrebt und somit den Wunsch nach Reputation hat, ist neben den Rahmenbedingungen vor allem von ihrer eigenen Zielfunktion abhängig. Wie gezeigt wurde, stellt eine inflationsaverse Bevölkerung nur dann eine hinreichende Bedingung für eine Stabilitätspolitik dar, wenn die Zentralbank primär Prestige erzielen will. Dann ist die Verfolgung einer Antiinflationspolitik sogar unabhängig vom Status der Zentralbank, d.h. in solch einem Fall erscheint die Unabhängigkeit gar nicht unbedingt erforderlich. Das wäre sie, wenn die Zentralbank als primäres Ziel ihre Reputation steigern will, da sie das Ziel dann leichter durchsetzen könnte. Je abhängiger die Zentralbank ist, desto mehr muß sie die Ziele der Regierung berücksichtigen. Ob diese primär ihre Reputation verfolgt, ist abhängig von ihren Wählern. Hier wäre eine inflationsaverse Bevölkerung wieder notwendig und unter Umständen hinreichend. Sollte die Zentralbank primär ihre Machtinteressen verfolgen, kann es aus stabilitätspolitischer Sicht sogar vorteilhaft sein, die Zentralbank nicht in die Unabhängigkeit zu entlassen. Solange sie bei einer stabilitätsbewußten Bevölkerung abhängig ist, wird sie zur Machtsteigerung ihr Prestige steigern müssen, was in solch einem Fall nur über den Aufbau von Reputation gelingt. Im Falle der Unabhängigkeit ist das Stabilitätsbewußtsein der Bevölkerung dann irrelevant, und nur die Inflationsaversion der Zentralbank bestimmt die Inflationsrate.

Gelangt man jedoch zu der Einsicht, daß Inflation in jedem Fall schlecht ist und deshalb bekämpft werden muß, sind zusätzliche Anreize nötig. Diese sind wiederum davon abhängig, welcher der Fälle vorliegt. Es gilt aber auch zu bedenken, daß Präferenzen sich ändern können und zukünftige Zentralbanker oder Bevölkerungen nicht die gleiche Stabilitätskultur haben wie die heutigen. In den Fällen 1, 3, 5, 7, 9, 10, 11, 12, 15 und 17 könnte man aus heutiger Sicht weitestgehend auf andere äußere Anreize verzichten, da die Zentralbank entweder sowieso stabilitätsorientiert ist oder zu solch einem Handeln ,gezwungen" wird. Äußere Anreize wären hier allerdings als eine Art Versicherung für 
die Zukunft zu interpretieren. Trotz sich ändernder Präferenzen könnte durch weitergehende Anreize die Durchführung einer an der Geldwertstabilität orientierten Geldpolitik auch für die Zukunft garantiert werden. Allerdings müßte in den Fällen 5, 7, 10, 15 und 17, in denen die Vermeidung von Inflation durch die Bevölkerung garantiert wird, für die Unabhängigkeit der Zentralbank gesorgt werden. Da die Anreize nur auf die Zentralbanker wirken können, müssen diese auch unabhängig von den Präferenzen der Bevölkerung handeln können.

In den Fällen 2, 4, 6 und 8, in denen die Zentralbank ihr Prestige steigern will, die Bevölkerung aber nicht inflationsavers ist, müssen externe Anreize dafür sorgen, daß es im Interesse der Zentralbank liegt, Reputation aufzubauen und dafür auch auf ihr eigentliches Ziel Prestige zu verzichten. Die Zentralbank muß dann ,gezwungen“ werden, der Bevölkerung ein Gut anzubieten, welches diese gar nicht wünscht, andererseits aber gut für sie ist.

Im dreizehnten und vierzehnten Fall sind externe Anreize für die Zentralbank absolut notwendig, da sie aufgrund gegebener Sicherheit ihre eigenen - nicht stabilitätsorientierten - Präferenzen durchsetzen kann. Die Zentralbank müßte hier „gezwungen“ werden, eine Antiinflationspolitik zu verfolgen. Im zehnten und sechzehnten Fall würde es aus heutiger Sicht reichen, der Zentralbank Unabhängigkeit zu gewähren. Nur dann wären die Präferenzen der Bevölkerung für sie unwichtig, und sie könnte ihrer Stabilitätsorientierung folgen (dies entspräche dann dem elften Fall). Aber auch hier muß bedacht werden, daß sich das Stabilitätsbewußtsein der Zentralbanker, z.B. durch Personalwechsel im Zentralbankrat, ändern kann. Externe Anreize wären wieder als eine Art Versicherung für die Zukunft zu verstehen. Der achtzehnte Fall schließlich erfordert eine unabhängige Zentralbank und externe Anreize auch schon aus heutiger Sicht, da die Zentralbanker nicht stabilitätsbewußt sind.

Es ist festzuhalten, daß eine an der Geldwertstabilität orientierte Geldpolitik in der Gegenwart und Zukunft letztlich nur gesichert ist, wenn die Zentralbank am Aufbau von Reputation interessiert ist. Da dies nicht immer gesichert ist, muß sie durch externe Anreize zum Reputationsaufbau „gezwungen“ werden. Damit ihr dies aber gelingen kann, ist es erforderlich, daß sie ihre Entscheidungen unabhängig von der Regierung und der Bevölkerung treffen kann, d.h. sie muß bei Verfolgung ihres Ziels unabhängig sein. Bei entsprechenden Rahmenbedingungen kann davon ausgegangen werden, daß jede Zentralbank primär nur noch ein Ziel, die Reputationssteigerung, verfolgt und ihre eigentlichen primären Ziele aufgrund der externen Anreize vernachlässigt. Zudem kann der Aufbau von Reputation dann auch gelingen, ohne daß eine Stabilisierungskrise 
herbeigeführt werden muß. Kennen die Wirtschaftssubjekte die externen Anreize zum Reputationsaufbau der Zentralbank, werden sie ihre Erwartungen mindern, und eine Antiinflationspolitik wird glaubwürdig. Die Realisation geringer Inflationsraten wird dann möglich, ohne dafür kurzfristig Arbeitslosigkeit zu produzieren.

\subsection{Anreize für Zentralbanker}

\subsubsection{Kontrollmechanismen}

Bevor im nächsten Abschnitt untersucht wird, welche expliziten Anreize die Zentralbanker zum gewünschten Verhalten veranlassen sollen, muß zunächst geprüft werden, ob dies Verhalten nicht schon durch die Existenz automatisch wirkender marktähnlicher oder politischer Kontrollmechanismen garantiert werden kann. Den Rahmen einer solchen Fragestellung stellt die PrinzipalAgent-Theorie dar. Sie geht davon aus, daß es zwischen Prinzipal und Agent wegen unterschiedlicher Zielfunktionen zu Interessengegensätzen kommt, die aufgrund der asymmetrischen Informationsverteilung nicht gelöst werden können. Es ist deshalb zu untersuchen, ob die zugrundeliegende institutionelle Ausgestaltung Kontrollmechanismen beinhaltet, die automatisch wirken können. Bei Abwesenheit solcher Mechanismen müssen externe Anreize die gewünschten Verhaltensweisen induzieren. In der Literatur werden die beiden Kontrollmechanismen ,exit" und ,voice“ unterschieden, die im folgenden näher ausgeführt werden. 52

Im Unternehmensbereich können die auftretenden Agency-Probleme abgemildert werden, indem marktähnliche Mechanismen den Agenten (Manager) dazu „Zwingen“, aus Eigeninteresse das Interesse des Prinzipals (Aktienbesitzer) zu verfolgen. Bei schlechter Leistung des Managers, ausgedrückt durch die realisierten Gewinne und Verluste, kann der Aktienbesitzer durch Verkauf seiner Anteile seine Unzufriedenheit zum Ausdruck bringen. Diese „Exit"-Möglichkeit verursacht dem Prinzipal nur geringe Transaktionskosten. Der Agent aber nimmt diese schlechte Bewertung seiner Leistung durch sinkende Kurse am Aktienmarkt wahr und ist dazu gezwungen, sein Verhalten zu ändern.

Genau diese Rückkoppelung fehlt für die Zentralbank. Zwar kann theoretisch jedes Individuum bei schlechter Politik der Zentralbank das Währungsgebiet verlassen, dies wird es in der Regel aber nicht tun, da die Austrittskosten unverhältnismäßig hoch wären. Selbst wenn das Individuum sich doch zum Aus-

52 Vgl. dazu Hirschman (1970). Die nachfolgende Argumentation für den Unternehmenssektor ist auf eine Aktiengesellschaft ausgelegt. Zur Prinzipal-Agent-Theorie vgl. z.B. Arrow (1985). 
tritt entschließen sollte, wird die Zentralbank dies nicht zum Anlaß nehmen, ihr Verhalten und somit ihre Politik zu ändern, da sie den Austritt nicht als Folge ihrer Politik bewerten würde.

Wenn im Rahmen der Zentralbankpolitik „exit“ als Straf- bzw. Kontrollmöglichkeit betrachtet wird, ist die Nutzung von Geld als Zahlungsmittel gemeint. Die Möglichkeit, seine eigene Währung als Wertaufbewahrungsmittel durch eine andere, „bessere“ zu ersetzen, ist zu relativ geringen Kosten möglich und wird auch in der Realität praktiziert. Das Argument, daß zwischen verschiedenen Zentralbanken keine marktähnliche Konkurrenz besteht, gilt zumindest nicht für Geld in seiner Funktion als Wertaufbewahrungsmittel. ${ }^{53} \mathrm{Ob}$ diese Form der Kontrollmöglichkeit aber schon ausreicht, um eine Zentralbank zu einer anderen Politik zu zwingen, muß bezweifelt werden, wenn man die Ergebnisse der Realität betrachtet.

Ein großes Problem für die automatische Kontrolle über den Absatzmarkt stellt auch die Tatsache dar, daß das von der Zentralbank angebotene Gut „Preisniveaustabilität" ein öffentliches Gut darstellt und somit niemand einen Anreiz besitzt, private Kosten auf sich zu nehmen, um die Zentralbank zu kontrollieren. Würde aus der realisierten Kontrolle tatsächlich ein „besseres“ Angebot der Zentralbank resultieren, käme dies allen Bürgern zugute und nicht nur denjenigen, die durch ihren Einsatz zu diesem besseren Ergebnis beigetragen haben. Genau diese eigennutzorientierte Sichtweise verhindert die Organisation einer Interessengemeinschaft für das Gut „Preisniveaustabilität““

Deshalb reagiert das einzelne Individuum auf geldpolitische Maßnahmen in erster Linie auch nicht als Kontrolleur, sondern als Besitzer von Vermögenswerten. Es wird sein individuelles Vermögen durch Umschichtung eher an die neuen Verhältnisse anpassen als Kosten für eine mögliche Kontrolle aufzuwenden. Eine Kontrollautomatik, wie sie für die Kapitalmärkte existiert, liegt für eine Zentralbank demnach nicht vor. Die Existenz von Kapitalmärkten und somit die kostengünstige Umwandlung von Vermögen erschwert sogar die Kontrolle der Zentralbank.

Eine weitere Kontrollmöglichkeit für den Unternehmensbereich stellen die internen und externen Arbeitsmärkte dar. So gewährleistet die Eigenkontrolle der Manager zumindest eine Minimaleffizienz. Bei schlechter Leistung müssen

Man muß sich nur die Beträge der Gelder ansehen, die täglich aus spekulativen Gründen gehandelt werden. Auch das Festkurssystems von Bretton Woods konnte die Spekulation nicht verhindern. Die D-Mark mußte mehrfach aufgewertet werden, weil der Markt sie höher bewertete als die Politiker. 
Manager befürchten, ausgewechselt zu werden. Dies stellt wiederum für Zentralbanker keine wirksame Drohung dar, weil ihre Amtsdauer in der Regel gesetzlich festgelegt ist und eine Änderung demzufolge einen entsprechenden Aufwand verursachen würde. Ein weiteres Problem für die Zentralbank stellt die Berufungspraxis der Zentralbankratsmitglieder dar. Diese werden im Rahmen eines politischen Entscheidungsprozesses bestimmt. Damit besteht zumindest die Gefahr, daß die politische Gesinnung mit eine Rolle spielt und die fachliche Qualifikation eher in den Hintergrund tritt.

Schließlich ist auch das Entlohnungssystem als Kontrollinstrument für die Zentralbank in der Regel nicht relevant. Die Zentralbanker erhalten ein leistungsunabhängiges Einkommen und müssen deshalb nicht befürchten, eine Minderung ihres Gehaltes hinnehmen zu müssen, sobald sie schlechte Ergebnisse erzielen. Ein Argument der Befürworter eines leistungsunabhängigen Einkommens ist die Ablehnung der Einkommensmaximierungshypothese für Zentralbanker. Nur nicht-monetäre Nutzenüberlegungen könnten die Zentralbanker dazu bewegen, auf weitaus höhere Einkommen zu verzichten, die sie in der Privatwirtschaft bei gleichen Qualifikationsanforderungen erzielen könnten. Somit stelle das Einkommen keinen Anreiz für die Zentralbanker dar, und demzufolge könne man sie darüber nicht sanktionieren.

Dieser Punkt wird hier jedoch angezweifelt, da die untergeordnete Bedeutung des Geldes nicht mit Sicherheit für jeden Zentralbanker unterstellt werden kann. Es wird bezweifelt, daß dieser Punkt allgemeingültig ist, und deshalb wird eine Spezifizierung erforderlich. Diese wird jedoch erst im Abschnitt 3.4.2.3 vorgenommen, wenn die möglichen externen Anreize diskutiert werden. Hier bleibt festzuhalten, daß das Entlohnungssystem in der bisherigen Praxis keinen Kontrollmechanismus für die Zentralbank darstellt, da es nicht leistungsbezogen konzipiert ist.

Nachdem die möglichen Kontrollautomatismen, die im Unternehmensbereich dazu führen, daß der Agent im Sinne des Prinzipals handelt, für die Zentralbank als nicht wirksam eingestuft werden mußten, bleibt noch die Prüfung eines Mechanismus, der für öffentliche Einrichtungen angeführt wird. Demnach könnte eine gewisse Disziplinierung erfolgen, wenn die Zentralbank durch das staatliche Budget finanziert würde, da dann eine direkte Verbindung zum Steuerzahler bestände. In diesem Fall wäre eine Zentralbank auch eher bereit zu reagieren, wenn einzelne Individuen ihre „Exit"-Möglichkeit wahrnehmen und das Währungsgebiet verlassen. Dies würde für die Zentralbank sonst den Verlust von Steuerzahlern bedeuten. Da Zentralbanken in der Regel aber ihre Auf- 
wendungen aus eigenen Erträgen finanzieren, wirkt auch dieser Kontrollmechanismus nicht. 54

Nachdem die Kontrollvariable „exit“ sich als für die Zentralbank nicht wirksam herausgestellt hat, muß noch geprüft werden, ob der Korrekturmechanismus ,voice“ wirkt. Es sind jetzt also nicht mehr automatisch wirksame marktähnliche Mechanismen gefragt, sondern diskretionär auftretende politische Aktivitäten.

Das Instrument Wahl als „Voice“-Kontrollvariable kommt bei einer unabhängigen Zentralbank nicht zur Anwendung, weil sie nicht direkt demokratisch legitimiert ist. Es besteht demnach auch keine direkte Beziehung zwischen der Zentralbank und den Wählern. Für die Zentralbank können nur andere politische Kontrollmechanismen relevant werden.

Ein Merkmal politischer Kontrollmechanismen ist die kollektive Organisierung von Individualinteressen, da politischer Druck nicht vom einzelnen Individuum ausgeübt werden kann, sondern nur von Interessengemeinschaften. Wie bereits ausgeführt wurde, wird sich keine Interessengemeinschaft zusammenfinden, die primär für die Preisniveaustabilität eintritt. Es werden also andere Gruppen sein, die Druck auf die Zentralbank auszuüben versuchen. ${ }^{55}$

Wie anhand der Inflationsanreize gezeigt wurde, hat die Inflation nicht für alle Wirtschaftssubjekte ausschließlich negative Effekte, sondern begünstigt einzelne Gruppen. Diese werden versuchen, politischen Druck auf die Zentralbank auszuüben und so individuelle Vorteile zu erzielen. Hier kommt wieder ein Unterschied zur Unternehmenstheorie zum Tragen. Während bei einer Aktiengesellschaft die Träger von Nutzen und Kosten identisch sind, sind sie es bei der Zentralbank nicht. Die Aktionäre erhalten nicht nur die Gewinne, sie müssen auch eventuelle Verluste tragen. Die Zentralbank könnte aber durch eine inflationäre Politik bestimmte Gruppen, die Druck auf sie ausüben, begünstigen, während die Kosten dieser Politik von der gesamten Währungsgemeinschaft zu tragen sind. Solange diese Kosten eine kritische Schwelle nicht über-

54 Die Wirksamkeit eines solchen Mechanismus wird hier ohnehin eher skeptisch beurteilt, da die Behörden, die durch das öffentliche Budget finanziert werden, sich in keiner Weise als vom Steuerzahler abhängig zu sehen brauchen. Es ist sehr zweifelhaft, ob eine Behörde effizienter arbeitet, nur weil sie vom Steuerzahler finanziert wird.

Zur Interdependenz zwischen der Zentralbank und Interessengruppen findet man in der Literatur nur wenig Hinweise. Insbesondere aber der Bankensektor und die Gewerkschaften haben ein Interesse an einer Einflußnahme auf die Zentralbankpolitik. Zu diesen Punkten vgl. Puchta (1981), S. 254-263. 
schreiten, werden die Kostenträger dieser Politik sich aber nicht zur Wehr setzen.

Eine geeignete Methode zur Beruhigung eventueller Wortführer sich formierender Gruppen stellt die Internalisierung dar. Als Wortführer der Zentralbank kritisch gegenüberstehender Gruppen können Ökonomen und Wirtschaftsjournalisten betrachtet werden. Da sie aber auf Informationen der Zentralbank angewiesen sind, um deren Handlungen zu beurteilen, kann eine geeignete Informationspolitik der Zentralbank zur Beeinflussung dieser Wortführer dienen. Noch weiter geht die Einbeziehung möglicher Kritiker in das Zentralbankgremium. Die Chance, selbst eine Position bei der Zentralbank zu besetzen, kann die Kontrollintensität beeinflussen. 56

Zusammenfassend läßt sich festhalten, daß weder die Kontrollvariable „exit“ noch der Mechanismus „,voice“ für die Zentralbank wirksam werden. Allein über die Kontrolle bzw. die nicht vorhandene Kontrolle kann eine unabhängige Zentralbank also nicht dazu gebracht werden, im Sinne des Prinzipals tätig zu werden, d.h. ausschließlich Preisniveaustabilität anzustreben. Es sind demnach doch externe Anreize zu setzen.

\subsubsection{Externe Anreize}

Man kann mehrere Formen der externen Anreize unterscheiden. Je nach Stärke der Beschränkung der Zentralbank lassen sie sich nach der Informiertheit der Bevölkerung, also aller Wirtschaftssubjekte eines Währungsgebiets, der Bindung an eine Regel oder, als schärfste Form, der direkten Anreize oder Sanktionen, die persönlich auf die Zentralbanker wirken, differenzieren. In diesem Kapitel werden nun nacheinander alle drei im Hinblick auf ihre Wirkung auf die Reputation untersucht.

\subsubsection{Abbau der Informationsasymmetrie}

Sobald die Annahme der symmetrischen Informationen aufgegeben wird und von einem Informationsvorsprung der Zentralbank, also des Agenten, ausgegangen wird, kann es zu Wohlfahrtsverlusten für die Gesellschaft kommen. Um diese zu minimieren, muß die Tätigkeit des Agenten kontrolliert werden. Die Grundlage jeder Kontrolle bilden aber Informationen. Da die Kontrolle selbst ein öffentliches Gut darstellt und die Informationsbeschaffung Kosten verursacht, kann die Zentralbank nahezu unkontrolliert handeln. Das einzelne Wirtschaftssubjekt geht nur von einer geringen Einflußnahme auf die Zentralbank 
aus und bewertet deshalb den eventuellen Nutzen einer Kontrolle gering. Da diesem aber andererseits hohe Kosten gegenüberstehen, wird es nur zu einer geringen Nachfrage nach Informationen kommen.

Informationen dienen aber dazu, eigene Präferenzen besser zu erkennen oder sie sogar zu verändern. Durch neue Informationen ändern sich die Rahmenbedingungen, und es kann modelltheoretisch zu völlig neuen Ergebnissen kommen. Die große Bedeutung von Informationen, vor allem die Möglichkeit der daraus resultierenden Präferenzänderungen, haben die Zentralbanken seit Anfang der siebziger Jahre systematisch genutzt, um so auf informellem Wege ihren Handlungsspielraum zu erweitern. Frei nach dem Motto „tue Gutes und sprich darüber" haben sie ihre Öffentlichkeitsarbeit verstärkt und den Wirtschaftssubjekten kostenlos Informationen zur Verfügung gestellt. Entsprechende Informationen aber können zur Prestigesteigerung beitragen, was wiederum die Position der Zentralbank stärkt. Eine positive Meinung der Bevölkerung über die Zentralbank stellt einen erheblichen Schutz vor politischen Pressionen dar. Selbst wenn die Unabhängigkeit einer Zentralbank nicht gewährleistet und sie politischem Druck ausgesetzt ist, wird dieser durch den Rückhalt der Bevölkerung zurückgehen oder zumindest auf indirektem Wege erfolgen müssen. Eine solche indirekte Schiene, auf der politische Interessen doch noch durchgesetzt werden können, ist z.B. die Besetzung des Zentralbankrats mit Politikern oder Fachkräften, die zumindest eine entsprechende parteipolitische Gesinnung aufweisen. ${ }^{57}$

Die einseitige Beeinflussung durch Informationen kann aber auch zur Ausnutzung der Macht führen. Ist die Zentralbank und somit der Agent der einzige Anbieter von Informationen, muß er nicht primär die Interessen des Prinzipals verfolgen, sondern kann seine Präferenzen durchsetzen und dem Prinzipal gleichzeitig suggerieren, daß er ausschließlich in dessen Interesse handele. Damit dieser Fall des eventuellen Informationsmißbrauchs nicht eintritt, muß dem Agenten zumindest vorgeschrieben werden, welche Art von Informationen er verbreiten muß. Dann tragen Informationen nicht nur dazu bei, den eigenen Handlungsspielraum zu erweitern, sondern können ihn auch begrenzen. In diesem Fall ist die Öffentlichkeitsarbeit keine freiwillige Entscheidung der Zentralbank mehr, sondern eine erzwungene. Da eine transparentere Geldpolitik eine Voraussetzung für deren Glaubwürdigkeit ist, sollte die Zentralbank ihre Beweggründe für ihre durchgeführten Maßnahmen - eventuell auch nicht er-

57 Dieser Fall wird hier aber ausgeschlossen, da an dieser Stelle die Forderungen des Kapitels 3.3.3 bereits erfüllt sein sollten. 
griffene Maßnahmen - der Öffentlichkeit erklären. Nur so wird das Vertrauen in die Geldpolitik gestärkt, und dies führt letztlich zur Reputationssteigerung.

Durch den Zwang zur Information auch über Zielverfehlungen wird der Handlungsspielraum der Zentralbank begrenzt, und die Kontrollmöglichkeit des Prinzipals nimmt zu. Ob allerdings die vermehrte Informationsverbreitung allein dafür sorgen kann, daß die Zentralbank ausschließlich am Ziel der Geldwertstabilität interessiert ist, muß bezweifelt werden. $58 \mathrm{Um}$ dies tatsächlich zu gewährleisten, müßte ihr Handlungsspielraum schon stärker eingeschränkt werden. Eine solche Möglichkeit kann die Einführung einer Regelbindung darstellen.

\subsubsection{Machtbegrenzung durch Regelbindung}

Die Frage, ob der geldpolitische Handlungsspielraum durch Einführung einer Regelbindung begrenzt werden soll, wird in der Wissenschaft schon sehr lange diskutiert. ${ }^{59}$ Unter einer Regelbindung wird dabei die strikte Einschränkung des Rechts zur geldpolitischen Entscheidung verstanden. Der diskretionäre Handlungsspielraum der Zentralbank wird komplett beseitigt, da die Geldpolitik nach einer Formel abläuft, die im voraus festgelegt wird. Die Glaubwürdigkeit der Geldpolitik wird quasi per Gesetz verordnet. Die Erwartungsunsicherheit der Wirtschaftssubjekte aufgrund fehlender Informationen über den aktuellen und zukünftigen Kurs der Geldpolitik ist nicht mehr gegeben, weil der Gesetzgeber den Geldpolitikern einen exakt definierten Handlungsrahmen vorgibt und ihnen so jeglichen diskretionären Entscheidungsfreiraum nimmt. Andererseits stellt eine Regelbindung aber auch einen Schutz vor politischen Pressionen dar. Reputationsüberlegungen spielen hier keine Rolle mehr, da die Reputation automatisch vorhanden ist. 60

Vollständige Diskretionarität hingegen liegt vor, wenn der Zentralbank nur das vage Ziel der Sorge für die ökonomische Wohlfahrt vorgegeben wird und sie Ermessensfreiheit im Hinblick auf die Formulierung ihrer ökonomischen Endziele, Zwischenziele und den Einsatz ihrer Instrumente besitzt.

58 Vgl. Diffenbach/Higgins (1987), S.16f., Filc (1994), S. 706ff., Frey (1981), S. 7, 278 und 291, Kastner (1994), S. 192, Kloten (1988a), S. 401 und Puchta (1981), S. 84.

59 Den Anstoß zu dieser Diskussion um „Regeln versus Diskretionarität“ in der Geldpolitik gab bereits Simon (1936). Im Rahmen dieser Arbeit soll aber nicht näher auf diese Kontroverse eingegangen werden. 
Die Erfahrung hat gezeigt, daß im Falle eines vollständig diskretionären Spielraums die Geldpolitik aufgrund von Time lags selbst zur Quelle ökonomischer Störungen werden kann. Deshalb wurde zum Zwecke der Stabilitätsorientierung die Einführung einer strikten Regelbindung gefordert. Diese Beseitigung des diskretionären Handlungsspielraums stellt den Kern des wirtschaftspolitischen Programms der Monetaristen dar. ${ }^{61}$

Unterstützt wird diese Negativbeurteilung in neuerer Zeit durch das Argument der Zeitinkonsistenz der optimalen Geldpolitik bei diskretionärem Handlungsspielraum der Politiker. Wie gezeigt wurde, führen strategische Überlegungen der Akteure zu einer suboptimalen Lösung für die Gesellschaft, und die Einführung einer Regelbindung könnte über die Erwartungsstabilisierung der Wirtschaftssubjekte zu einem besserem Ergebnis führen.

Es kann jedoch gezeigt werden, daß bei Auftreten realer Schocks die Regelbindung wegen des Fehlens der notwendigen Flexibilität zu schlechteren Ergebnissen führt als eine diskretionäre Politik. ${ }^{62}$ Andererseits kann letztere aber nicht zur Stabilisierung der Erwartungen beitragen. Dies kann nur eine stetige Geldpolitik erreichen, weshalb prinzipiell eine Regelbindung erforderlich ist. $\mathrm{Da}$ die Wirtschaftssubjekte einen überraschenden Schock aber als solchen erkennen, kann die Zentralbank in diesem Fall einem Beschäftigungsrückgang entgegenwirken, ohne daß dies ihre Reputation zwangsläufig mindert. Die Regelbindung sollte also Spielraum lassen, um bei unvorhersehbaren Ereignissen reagieren zu können.

In der Literatur findet man eine Vielzahl von Vorschlägen zur Regelbindung. ${ }^{63}$ Dennoch ist es bisher nicht gelungen, eine allgemeingültige optimale Regel zu entwickeln. Sobald aber unzureichende Regeln angewendet werden, besteht die Gefahr der Politisierung der Regelanpassung. Tritt eine Störung auf, die bei der Regelformulierung nicht berücksichtigt wurde, muß es zur Neuformulierung oder Spezifizierung der Regel kommen. Dies aber kann politisch ausgenutzt werden und erschüttert deshalb nur das Vertrauen und somit die Glaubwürdigkeit in die Geldpolitik.

61 Friedman fordert die Einführung einer starren Geldmengenregel, die als k-ProzentRegel bekannt geworden ist. Vgl. Friedman (1959).

62 Zum formalen Beweis vgl. Persson/Tabellini (1991), S. 21ff. und Clausen/Willms (1993), S. 607.

63 Diese sollen hier aber nicht im einzelnen diskutiert werden. Einen Überblicksartikel zu verschiedenen Regelbindungen gibt z.B. Wagner (1992), S. $133 \mathrm{ff}$. 
Zudem müssen auch die Kosten einer Regelbindung berücksichtigt werden. Insbesondere die Implementierungs- und Kontrollkosten sind hier zu nennen. Es muß hinterfragt werden, ob von seiten der Politiker überhaupt ein Anreiz besteht, eine Regelbindung einzuführen, da dies auch deren Profilierungsverlangen widerspricht. Kommt es dennoch zur Regeleinführung, muß sichergestellt werden, daß diese eingehalten wird, was wiederum eine wirksame Kontrolle erforderlich macht. ${ }^{64}$

Wichtiger als die explizite Formulierung einer Regel ist zur Stabilisierung der Erwartungen aber die Verstetigung der Geldpolitik. Nicht die Beseitigung des Ermessensspielraums der Zentralbanker ist erforderlich, sondern nur die Berechenbarkeit ihrer Handlungen. Ein Mittel dafür stellt die Vorankündigung eines Geldmengenziels dar. Dies kann als Signal für die Wirtschaftssubjekte dienen und vertrauensbildend wirken. Die anderen Akteure können die eigenen Handlungen dann auf die beabsichtigte Geldpolitik abstimmen. So wird auch eine effizientere Planung finanzpolitischer Maßnahmen möglich, weil die monetären Restriktionen im voraus bekannt sind. Zudem stellt es eine Selbstbindung der Zentralbank dar mit der Folge, daß Zielverfehlungen öffentlich begründet werden müssen. Faktisch liegt für die Zentralbank eine Beschränkung ihres Handlungsspielraums vor. Gleichzeitig entkräftet diese Konkretisierung ihres Handlungsspielraums auch das staatspolitische Argument gegen eine unabhängige Zentralbank, so daß die Selbstbindung eine wichtige Unterstützungsfunktion für die De-facto-Autonomie hat. Durch die vorherige öffentliche Bekanntgabe ihres zukünftigen geldpolitischen Kurses wird die Zentralbank auch besser überprüfbar, und die Möglichkeit zur Kritik an ihrer Politik steigt.

Bei der Wahl der geldpolitischen Steuerungsgröße ist jedoch zu berücksichtigen, daß die folgenden vier Kriterien erfüllt sind: das Endzielkriterium, das eine direkte Verbindung der Steuerungsgröße zum Endziel fordert, das Anreizkriterium, nach welchem zu hinterfragen ist, ob die angekündigte Steuerung eingehalten wird, das Kontroll- sowie das Bestandskriterium. Entsprechend dem Kontrollkriterium müssen Entscheidungs- und Verantwortungsbereich identisch sein, also eine eindeutige Schuldzuweisung ermöglichen, während nach dem Bestandskriterium eine einmal festgelegte Regel bzw. hier festgelegte Steuerungsgröße nicht wieder abgeschafft wird, sobald ihre Einhaltung zu Problemen führt. ${ }^{65}$

Vgl. Kastner (1994), S. 226. Den Kostenaspekt für unterschiedliche Regelbindungen untersucht Hamacher (1995), S. 50ff. 
Die Ankündigung eines angestrebten Ziels bzw. Zwischenziels ist aber nur zweckmäßig, wenn sie auch glaubwürdig ist. Nur wenn die Wirtschaftssubjekte davon ausgehen können, daß die Zentralbank tatsächlich so handelt, wie sie es vorher angekündigt hat, werden sie dies entsprechend in ihren Erwartungen berücksichtigen. Wenn Erfahrungen mit Ankündigungen fehlen und die Wirtschaftssubjekte somit ihre Erwartungen nicht in Abhängigkeit davon wählen können, ob die Zentralbank frühere Versprechungen eingehalten hat oder nicht, kann eine Determinante für die Glaubwürdigkeit einer solchen Ankündigung das Vertrauen in die Person(en) sein, die die Zentralbank vertritt bzw. vertreten. Da solch eine Bewertung aber nur aus subjektiver Sicht der Bevölkerung erfolgen kann, ist es besser, eine Ankündigung sofort so glaubwürdig zu machen, daß sie aus objektiver Sicht realisierbar ist. Dies erfolgt dadurch, daß die Kosten einer Nichteinhaltung der Ankündigung erhöht werden. Wenn die Kosten der Abweichung von der Ankündigung und somit der Zwang zur Einhaltung genauso hoch oder höher sind als der Anreiz, von ihr abzuweichen, kann eine Abweichung ausgeschlossen werden. Nur dann erscheint die Einhaltung der Ankündigung auch ex post noch optimal.

Letztlich ist also eine explizite Sanktionierung erforderlich, die zudem automatisch ausgelöst werden muß. Androhungen von potentiellen Bestrafungen bei Nichteinhaltung der Ankündigung, die letztlich doch nicht vorgenommen werden, reichen nicht aus, um eine Ankündigung glaubwürdig zu machen. Die Wirtschaftssubjekte verlassen sich bei ihrer Erwartungsbildung nicht auf bloße Bekenntnisse der geldpolitisch Verantwortlichen, sie verlangen stärkere Garantien in Form handfester Sicherungen gegen Fehlleitungen der Geldpolitik. ${ }^{66}$

\subsubsection{Persönlich wirkende Anreize und Sanktionen}

In neuerer Zeit werden vereinzelt immer wieder persönlich wirkende Sanktionen für Zentralbanker gefordert, damit diese ihr primäres Ziel „Vermeidung von Inflation“, also „Aufbau von Reputation“, auch verfolgen.67 Gefordert wird allerdings immer eine Kollektivstrafe für den gesamten Zentralbankrat oder alternativ ausschließlich die Bestrafung des Präsidenten. Als Begründung wird angeführt, daß für einen Außenstehenden die Entscheidungsfindung im

66 Vgl. Caesar (1981), S. 119, Caesar (1982), S. 576, Francke (1990), S. 143ff., Frey (1981), S. 30f., Huß/Trapp (1982), S. 85, Issing (1992b), S.51f., Kloten (1988a), S. 404f., Kösters (1990), S.129, Kösters (1991), S. 160ff., Neumann (1988), S. 48, Neumann (1992), S. 63 und 70ff., Puchta (1981), S. 292f. und Willms (1990b), S. 555 .

67 In einigen Zentralbankstatuten wurden solche Forderungen sogar schon explizit eingebaut. Als Pionier auf diesem Gebiet gilt die Zentralbank von Neuseeland. 
Zentralbankrat nicht nachvollziehbar sei. Das Problem ist jedoch zu lösen, indem Sitzungsprotokolle angefertigt und veröffentlicht werden, die genau erfassen, wer wie abgestimmt hat. Dann wird ersichtlich, welche einzelnen Mitglieder für eine eventuell schlechte Leistung verantwortlich sind und deshalb „,bestraft" werden müssen. Zumindest bei einer sehr heterogenen Gruppe von Zentralbankern ist nicht einzusehen, daß alle bzw. nur der Präsident bestraft werden, vor allem wenn einzelne sogar explizit gegen bestimmte Maßnahmen, die das schlechte Ergebnis verursachten, gestimmt haben, sich aber der Mehrheitsentscheidung fügen mußten. Die Bewertung nur der eigenen Leistung kann selbst schon einen Anreiz darstellen, sich für das Stabilitätsziel einzusetzen.

Ein weiteres Problem in der Literatur ist darin zu sehen, daß zwar allgemein pekuniäre Anreize nicht für notwendig gehalten werden, weil schon nicht-pekuniäre die gleiche Funktion erfüllen könnten, diese werden aber kaum konkretisiert, und auch die notwendigen Voraussetzungen werden nicht explizit herausgestellt. Ein Argument gegen pekuniäre Anreize ist, daß es nicht die Bezahlung sei, die die Zentralbanker dazu motiviere, eine solche Position zu übernehmen, da in der Privatwirtschaft weitaus höhere Einkommen erzielbar sind. Die Gültigkeit dieses Arguments kann aber nicht für jeden einzelnen Zentralbanker unterstellt werden. Gewiß gibt es Beispiele für Zentralbanker, die auf Angebote mit höheren Gehältern verzichtet haben ${ }^{68}$, ob dies aber der Regelfall ist, kann bezweifelt werden. Für viele Mitglieder des Zentralbankrats ist die Berufung mit einer deutlichen Verbesserung ihres Einkommens verbunden, und demnach kann die Entscheidung, zur Zentralbank zu gehen, durchaus finanziell motiviert sein. Die höhere Bezahlung der gleichen Leistung in der Privatwirtschaft bedeutet auch nicht, daß die berufenen Zentralbanker entsprechende Angebote erhalten hätten.

Es soll nun aufgezeigt werden, welche Sanktionsmöglichkeiten existieren, um vom Agenten das gewünschte Ergebnis zu erhalten. Welche Sanktionsmöglichkeit jeweils relevant ist, hängt dabei im wesentlichen von der Zielfunktion des einzelnen Zentralbankers ab. Die Sanktionen und Anreize sind also nur bestimmbar, wenn die Nutzenüberlegungen der Agenten berücksichtigt werden. Die für Zentralbanker möglichen Nutzenelemente wurden im vorherigen Kapitel diskutiert. Sie dienen jetzt als Grundlage für die zu untersuchenden Anreizstrukturen.

Wie ausgeführt wurde, werden für Zentralbanker im wesentlichen die fünf möglichen Zwischenziele Einkommen, Sicherheit, Prestige, Reputation oder 
Macht unterstellt, durch deren Erfüllung sie ihren Eigennutz maximiert sehen. Dabei wurde davon ausgegangen, daß jeweils ein Ziel besonders wichtig ist, um den jeweils subjektiven Eigennutz eines Zentralbankers zu optimieren.

Unterstellt man zunächst, daß das primäre Ziel eines Zentralbankers in der Erzielung von Einkommen liegt, sind pekuniäre Anreize am besten geeignet, das erwünschte Ziel zu erreichen. Gefordert wird hier also ein leistungsbezogenes Einkommen, welches sowohl aus positiven Anreizen für gute Leistungen als auch aus negativen Sanktionen in Form von Gehaltskürzungen bei schlechten Leistungen bestehen kann.

In der Literatur werden hierzu sowohl Nominallohn- als auch Reallohnkürzungen diskutiert. Der Vorschlag zur Nominallohnkürzung sieht vor, daß das nominale Gehalt gemindert werden soll, sobald die Preissteigerungsrate über einen bestimmten Zeitraum eine vorher im Gesetz festgelegte Inflationsrate übersteigt. Diese Forderung kann aber zu einer zu restriktiven Politik führen, weil die Zentralbanker versuchen könnten, auch von ihnen nicht zu verantwortende Inflationstendenzen zu bekämpfen. Deshalb knüpft ein abgemilderter Vorschlag einer Einkommenseinbuße am Realeinkommen an. Die Zentralbanker sollen nicht bestraft werden, indem ihre Einkommen nominal gekürzt werden, sondern indem die Gehälter nicht an das Preisniveau gekoppelt werden. Durch das explizite Verbot zur Indexierung der Gehälter besteht ein Anreiz zur Stabilitätspolitik, da die Zentralbanker sonst einen Reallohnverlust erleiden. Allerdings kann auch diese Form der Sanktionierung eine Rezession verursachen, da auch hier der Anreiz zur Durchführung einer „zu restriktiven“ Geldpolitik bestehen kann, wenn eine importierte oder nicht von der Zentralbank verschuldete Inflation beseitigt werden soll. Letztlich sind die Konsequenzen für die Wirtschaftsentwicklung unabhängig davon, welche der beiden Sanktionsformen gewählt wird. Einen effektiveren Anreiz stellt aber die erste Form dar, weil hier ein Versagen explizit sichtbar wird, welches durch eine Einkommensminderung bestraft wird.

Um die negativen Effekte auf die Wirtschaft zu vermeiden, müßte ein geeigneter Zeithorizont für die Bewertung der Leistung gewählt werden. Es besteht kein Zweifel, daß die Inflationsrate kurzfristig auch von der Tarif- und Fiskalpolitik mitbestimmt wird. Nur langfristig ist davon auszugehen, daß die Zentralbank die Inflationsrate bestimmen kann, da langfristig jede Inflation zu finanzieren ist, und das Geldangebot liegt in der Hand der Zentralbank. Es sollte demnach nicht zur Bestrafung der Zentralbanker kommen, wenn kurzfristig positive Inflationsraten realisiert werden. Als Grundlage sollte hier ein mehrjähriger Durchschnitt dienen, da dann eher als in der kurzen Frist davon ausgegan- 
gen werden kann, daß die Inflationsrate von der Zentralbank verursacht wurde. Es kann allerdings argumentiert werden, daß die Inflationsrate in jedem Jahr wieder von einem externen Effekt dominiert werden kann, den die Zentralbanker nicht zu vertreten haben. Dieser Fall ist nicht auszuschließen, kann aber als allgemeines Berufsrisiko behandelt werden. Auch Manager in der Privatwirtschaft werden für Ergebnisse sanktioniert, die sie eventuell nicht zu vertreten haben. Dieses Risiko einer ungerechten Bestrafung wird durch ein überdurchschnittliches Einkommen einschließlich einer Risikoprämie abgegolten. Entsprechend sollten auch Zentralbanker einen Risikozuschlag zu ihrem Gehalt bekommen. ${ }^{69}$

Wie die pekuniäre Bestrafung im einzelnen aussehen soll und über wieviel Jahre eine Erfolgsbetrachtung vorzunehmen ist, soll hier im Detail nicht erörtert werden. Wichtig ist nur, daß die Androhung einer Einkommenseinbuße bei entsprechender Zielfunktion einen bedeutenden Anreiz darstellen kann, um die Zentralbanker zu stabilitätsbewußtem Verhalten zu animieren, sich also doch Reputation aufzubauen.

Neben diesen pekuniären Anreizen können aber auch nicht-pekuniäre Anreize wirksam werden, wenn die einzelnen Zentralbanker ihren Nutzen durch entsprechend andere Faktoren maximieren. Ist die Sicherheit ihr primäres Ziel, kann man diese wirksam durch die Drohung beeinflussen, entweder ihre Amtszeit bei schlechter Leistung zu verringern oder sie gleich zu entlassen. Dieser letzte Vorschlag stellt eine harte, damit aber auch wirksame Drohung dar. Müssen Zentralbanker bei Zielverfehlungen den Verlust ihres Arbeitsplatzes befürchten, werden sie aufgrund ihres Sicherheitsstrebens eine Antiinflationspolitik verfolgen und sich somit doch Reputation aufbauen.

Teilweise wird das Ziel Sicherheit in der Literatur auch als Sicherung des Status der Zentralbank interpretiert. Dies Ziel kann nur für unabhängige Zentralbanken gelten, da es nicht im Interesse einer abhängigen Zentralbank liegen wird, ihren Status zu sichern. Dann könnte die Unabhängigkeit insofern als Anreiz dienen, als die Zentralbank Konflikte mit dem Parlament vermeiden müßte, da sonst die Gefahr einer Gesetzesänderung bestände. ${ }^{70}$ Die Disziplinierung der Zentralbank durch das Parlament über die stets drohende Gefahr des Verlustes der Unabhängigkeit würde die Zentralbank doch wieder von wahlorientierten Politikern abhängig machen. Gerade die Abhängigkeit würde sie daran hindern, den Aufbau von Reputation als ihr primäres Ziel zu sehen.

69 Vgl. hierzu Puchta (1981), 324f.

70 Zu diesem Punkt vgl. Filc (1994), S. 705ff. 
Ein anderes nicht-monetäres Ziel eines Zentralbankers kann die Erzielung von Prestige sein. Für diesen Fall ist zunächst zu klären, bei wem man überhaupt beliebt sein will. In der Regel wird davon auszugehen sein, daß der Adressat die heimische Bevölkerung ist. Ob der Aufbau von Prestige nun über den Aufbau von Reputation geschieht oder gerade nicht, ist somit abhängig von der Inflationsmentalität der Bevölkerung.

Im Optimalfall kann ein Zentralbanker sein persönliches Ziel, die Erzielung von Prestige, nur über den Aufbau von Reputation und somit Verfolgung einer strikten Antiinflationspolitik realisieren. Bedingung dafür ist jedoch eine ausgeprägte Inflationsaversion der Bevölkerung. Ist diese hingegen nicht gegeben und ein Zentralbanker wird mit Prestige belohnt, wenn er gerade auf eine strikte Stabilitätspolitik verzichtet und statt dessen zur Realisation anderer wirtschaftspolitisch gewünschter Ziele beiträgt, müssen „zum Wohle des Volkes“ dem Zentralbanker explizite Anreize gesetzt werden, damit sich dieser Reputation aufbaut.

Es sind Sanktionen gesucht, die dazu führen, daß der Zentralbanker sein eigentlich primäres Ziel zugunsten des Reputationsaufbaus aufgibt. Eine solche Möglichkeit besteht darin, daß es dem Zentralbanker unmöglich wird, Prestige zu erhalten, selbst wenn er sich so verhält, wie es die Bevölkerung wünscht. Muß er befürchten, daß er im Falle einer Zustimmung zu stabilitätswidrigen Entscheidungen sein Stimmrecht zeitweise verliert und somit von zukünftigen Entscheidungen ausgeschlossen wird, kann er davon ausgehen, daß dies seinem Prestige schadet. Die Wirtschaftssubjekte wissen dann eventuell, daß der Zentralbanker zwar genau die gleichen Zielvorstellungen hat wie sie selbst, diese jedoch nicht umsetzen kann. Da die Erzielung von Prestige aber eher mit dem „Können“ als dem „Wollen“ der Durchführung bestimmter Handlungen verbunden ist, kann davon ausgegangen werden, daß der kurzfristigen Prestigesteigerung durch die Vernachlässigung des Stabilitätsziels schon schnell eine Prestigeeinbuße durch den Verlust des Stimmrechts folgt.

Sollte dies jedoch nicht der Fall sein und der Zentralbanker trotz der Verfehlung seines primären Ziels sich nicht veranlaßt sehen, seine Reputation zu verbessern, bleibt letztlich als weitestgehender Anreiz noch die Drohung der Entlassung. Diese Sanktion führt mit großer Wahrscheinlichkeit zum gewünschten Verhalten. Statt eines solchen persönlich wirkenden Anreizes könnte allerdings auch eine entsprechende Rahmenvorschrift für den gesamten Zentralbankrat zur gewünschten Zielrealisation führen. Würde eine expansive geldpolitische Maßnahme nicht nur eine Mehrheit, sondern Einstimmigkeit erfordern („Veto für die Stabilität"), könnte dies die Wahrscheinlichkeit für stabilitätswidriges 
Verhalten zumindest reduzieren. Zum Problem kann allerdings auch diese Regelung werden, wenn der gesamte Zentralbankrat aus nichtstabilitätsbewußten Individuen besteht oder es den eher inflationsverharmlosenden Mitgliedern gelingt, die anderen zu überzeugen, stabilitätswidrig abzustimmen. Wieder wäre hier als letztes Mittel die Entlassung zu sehen, in diesem Fall sogar des gesamten Zentralbankrats.

Der andere Extremfall wäre gegeben, wenn alle oder auch nur ein Zentralbanker den Aufbau von Reputation als primäres Ziel haben. Im letzteren Falle muß zusätzlich die Forderung nach Einstimmigkeit der Beschlüsse expansiver Maßnahmen im Zentralbankrat erfüllt sein. Wenn der Reputationsaufbau als persönliches Ziel des Agenten gesehen werden kann, liegt Interessenharmonie mit dem Prinzipal vor, und es sind keine zusätzlichen Anreize erforderlich. Der Agent würde dann ein schlechtes Ergebnis in Form positiver Inflationsraten schon als persönlichen Mißerfolg betrachten und demzufolge auch ohne Androhung von Sanktionen das gewünschte Ergebnis zu realisieren versuchen.

Als letzte Möglichkeit zur Nutzenmaximierung des Zentralbankers wurde die Erzielung und Ausübung von Macht genannt. Es wird teilweise argumentiert, daß die strikte Durchführung einer an der Stabilität orientierten Geldpolitik schon die Ausübung von Macht bedeutet. Mit einer strengen Antiinflationspolitik kann die Zentralbank der Regierung und den Tarifpartnern (hier primär den Gewerkschaften) ihre Macht demonstrieren, indem sie über deren realen Ausgabenspielraum entscheidet. Trotz anderer Vorstellungen der Politiker oder Gewerkschafter kann die Zentralbank durch Setzen des Geldangebots ihre Interessen durchsetzen und die anderen Akteure zu einem stabilitätsgerechten Verhalten zwingen.

Diese Argumentation kann theoretisch aber auch entgegengesetzt laufen, daß nämlich die Regierung inflationsavers ist und die Zentralbank ihre Macht demonstriert, indem sie positive Inflationsraten zuläßt. Um dieses Szenario zu vermeiden, ist es dann doch wieder erforderlich, Anreize zum Aufbau von Reputation zu setzen. Diese müßten so aussehen, daß im Falle eines stabilitätswidrigen Verhaltens die Macht des Zentralbankers beschnitten wird, da er so seinen persönlichen Nutzen gemindert sieht.

Eine Machteinschränkung stellt es dar, wenn im Falle eines Fehlverhaltens des Zentralbankers diesem das Stimmrecht entzogen wird, selbst wenn es nur kurzfristig erfolgt. Alternativ könnte sein Aufgabenbereich beschränkt werden. Dies könnte dadurch erfolgen, daß er für weniger Aufgaben zuständig ist oder aber durch Einführung des Vier-Augen-Prinzips. Letzteres würde bedeuten, 
daß er keine Entscheidung mehr allein fällen darf, sondern von mindestens einem Partner unterstützt werden muß. Eine solche Einschränkung stellt für jemanden, der primär seine persönliche Macht maximieren will, eine empfindliche Störung dar und kann somit als wirksamer Anreiz gesehen werden, ihn zu einem geforderten Verhalten zu zwingen. Eine andere Möglichkeit der Machtbeschränkung wäre wieder die Entlassung bei Mißerfolg. Diese Sanktion stellt auch hier die schärfste Form eines Anreizes dar. ${ }^{71}$

Zusammenfassend bleibt festzuhalten, daß die Drohung der automatischen Entlassung bei mehrjähriger Zielverfehlung unabhängig vom primären Ziel des Zentralbankers immer einen wirksamen Anreizmechanismus zum Aufbau von Reputation darstellt. Nur wenn der Wunsch nach Reputation ohnehin das Ziel des Zentralbankers ist, kann auf diesen oder andere Anreize verzichtet werden. Sobald aber ein anderes Zwischenziel zur Nutzenmaximierung des Zentralbankers führt, muß es einen Anreizmechanismus geben, der zudem automatisch wirkt. Wenn erst noch politisch bestimmt werden muß, ob ein Versagen überhaupt vorliegt bzw. ob dies so eklatant ist, daß es bestraft werden muß, ist das ganze Anreizsystem wirkungslos, da Politiker häufig zu Kompromissen bereit sind.

Andererseits wird ein nach Reputation strebender Zentralbanker externe Anreize nicht ablehnen, sie im Gegensatz zu seinen Kollegen, die andere Ziele verfolgen, sogar begrüßen, da Anreize der Reputation förderlich sind. Wissen oder glauben die Wirtschaftssubjekte nicht, daß ein Zentralbanker primär seine Reputation steigern will, werden sie spätestens durch die Existenz der externen Anreize ihre Erwartungen für die Zentralbank gering wählen. Die externen Anreize sind hier als vertrauensschaffende $\mathrm{Maßnahme}$ zu interpretieren. ${ }^{72} \mathrm{Die}$ Bereitschaft zur persönlichen Haftung durch Verlust des Arbeitsplatzes bei Mißerfolg steigert das Vertrauen der Wirtschaftssubjekte gegenüber den Zentralbankern, welches durch geringe Inflationserwartungen zum Ausdruck kommt. Eine Antiinflationspolitik wird also glaubwürdiger. Das Ergebnis der persönlichen Anreize kann demnach nicht nur der Wunsch des Zentralbankers nach Reputation sein, sondern auch die Möglichkeit, Reputation zu erlangen, ohne der Gesellschaft Kosten in Form von Arbeitslosigkeit aufzubürden. Die

71 Vgl. Kösters (1991), Neumann (1990), (1992), Vaubel, (1989), (1990), (1993) und Willms (1990b).

72 Wie die persönliche Haftung eines Unternehmers sein Unternehmen aus Sicht der Banken kreditwürdiger werden läßt im Gegensatz z.B. zu einer nur beschränkt haftenden $\mathrm{GmbH}$, führen die persönlichen Anreize für die Zentralbank auch zur Verbesserung ihrer Kreditwürdigkeit, welche als Vertrauen zu ihr zu sehen ist und in geringen Inflationserwartungen der Wirtschaftssubjekte zum Ausdruck kommt. 
Reputation kann dann schneller und leichter (wegen der fehlenden Anpassungskrise) gesteigert werden.

\subsubsection{Die Bedeutung für das Modell}

Die Reputation der Zentralbank ist um so größer, je höher der von der Zentralbank zu kontrollierende Teil der Inflationsrate ist. Entsprechend Gleichung (2.25) hängt dieser Teil der tatsächlichen Inflationsrate vom Stabilitätsbewußtsein der Zentralbank sowie von ihrer Zeitpräferenzrate ab. Aber auch die Meinung der Wirtschaftssubjekte bezüglich der Gewichtung dieser Größen von seiten der Zentralbank ist wichtig, da diese die Inflationserwartungen bestimmen und somit über die Glaubwürdigkeit der Geldpolitik entscheiden. Letztere wiederum bestimmt die Kosten einer Antiinflationspolitik. Wie gezeigt wurde, kann die Zentralbank ihre angekündigte Inflationsrate, die sie entsprechend ihren Präferenzen berechnet, nur realisieren, wenn die Wirtschaftssubjekte diese für glaubwürdig halten. Sind die Ankündigungen für die Wirtschaftssubjekte jedoch unglaubwürdig, muß die Zentralbank bei der Realisation ihrer optimalen Inflationsrate die höheren Erwartungen berücksichtigen, da ein Unterschreiten des erwarteten Wertes eine Überraschungsdeflation bedeutet mit der Folge gesellschaftlicher Kosten in Form von Arbeitslosigkeit.

Werden der Zentralbank wirksame externe Anreize gesetzt, indem jeder Zentralbanker mit persönlichen Nachteilen zu rechnen hat, wenn er sich nicht stabilitätskonform verhält, kann mit großer Wahrscheinlichkeit davon ausgegangen werden, daß jeder Zentralbanker seine Reputation steigern will. Die Reputation ist dann quasi automatisch festgelegt. Die Zentralbanker werden die Werte für a und q nahe dem Maximalwert Eins wählen, um keine persönlichen Nachteile durch hohe Inflationsraten in Kauf nehmen zu müssen, und die Wirtschaftssubjekte können darauf vertrauen, daß die Zentralbank die optimale Inflationsrate auch tatsächlich realisieren will. Der Anreiz zur Inflationierung bei einer von den Wirtschaftssubjekten erwarteten Inflationsrate von Null wird überkompensiert durch Kosten, die jeden Zentralbanker persönlich treffen. Sind diese Kosten hoch genug, wovon spätestens bei der Androhung mit Entlassung ausgegangen werden kann, haben die Zentralbanker keinen Anreiz mehr zu einer Überraschungsinflation. Eine Antiinflationspolitik wird glaubwürdiger, und die Inflationserwartungen werden sinken.

Wenn davon ausgegangen werden kann, daß die Kosten für die Zentralbanker bei einem Verstoß tatsächlich wirksam werden, wird die optimale Inflationsrate mit hoher Wahrscheinlichkeit realisiert. Geeignete externe Rahmenbedingungen für die Zentralbank können also dazu führen, daß sie eine Reputation 
nahe ihrem Optimalwert genießt bzw. ihn sogar erreicht. Wie gezeigt wurde, führt dies dann dazu, daß die Zentralbank tatsächlich eine geringe Inflationsrate realisieren kann. Dies muß auch kurzfristig nicht mit Beschäftigungs- oder Wachstumseinbußen erkauft werden, sondern ist ohne gesellschaftliche Kosten möglich, weil die Antiinflationspolitik der Zentralbank glaubwürdig ist. 
Iris Henning - 978-3-631-75125-1

Downloaded from PubFactory at 01/11/2019 08:02:27AM

via free access 
Die Reputation der Europäischen Zentralbank (EZB) Die Leistung der potentiellen Mitgliedsländer

Im Rahmen dieser Arbeit wird nicht davon ausgegangen, daß die EZB die Reputation der besten Zentralbank einfach übernehmen kann. Es erscheint unrealistisch, daß eine Zentralbank, die sich gleichberechtigt aus mehreren Partnern zusammensetzt, nur von der Reputation bestimmt wird, die ein Partner genießt. Zwar kann die Reputation durch entsprechende Rahmenbedingungen, wie sie im theoretischen Teil dieser Arbeit diskutiert wurden, positiv beeinflußt werden, an dieser Stelle soll jedoch zunächst nur die erbrachte Leistung als Indikator untersucht werden. Die EZB wird allerdings erst neu gegründet und demzufolge existieren für sie keine empirischen Daten. Es ist deshalb erforderlich, die Leistung aller nationalen Zentralbanken zu betrachten, die eventuell Mitglied der EZB werden könnten. Geht man davon aus, daß alle Mitglieder des Europäischen Währungssystems (EWS) sowie Griechenland als Mitglied der Europäischen Union (EU) eine Teilnahme an der EZB wünschen, sind deren vergangene Leistungen relevant.

Aufgrund der kurzfristigen Beeinflussung der Inflationsrate durch die Fiskalund Lohnpolitik stellt die kurzfristige Inflationsentwicklung nur einen unsicheren Indikator für das Verhalten der Zentralbank dar. Eine engere Bindung zwischen Geldpolitik und Inflationsrate kommt in der langfristigen Inflationsrate zum Ausdruck, da jede Inflation zu finanzieren ist. Tabelle 4.1 zeigt in Spalte 2 die durchschnittlichen Inflationsraten der einzelnen Länder im Zeitraum von 1957 bis 1995.1 Diese Werte können erste Anhaltspunkte für die Reputation der einzelnen Zentralbanken liefern.

Danach hat die Bundesbank das beste Ergebnis erzielt. Allein aufgrund dieses Kriteriums hätte die Bundesbank die höchste Reputation. Es muß jedoch berücksichtigt werden, daß die Zentralbanken im betrachteten Zeitraum von 1957 bis 1971 aufgrund des Festkurssystems von Bretton Woods keine autonome Geldpolitik durchführen konnten. Dies zeigt sich auch in den realisierten Inflationsraten. Wie aus Spalte 3 der Tabelle zu erkennen ist, beträgt der Abstand zwischen der geringsten ( $2,1 \%$ in Griechenland) und der höchsten Inflationsrate $(6,8 \%$ in Spanien) ,nur“ 4,7 Prozentpunkte. Die tatsächliche Leistung der Zentralbanken zeigt sich aber eher bei flexiblen Wechselkursen. Deshalb enthält die Tabelle in der vierten Spalte die durchschnittlichen Inflationsraten der Länder für einen Zeitraum, der überwiegend durch flexible Wechselkurse

11957 wird hier als Ausgangsjahr gewählt, weil in diesem Jahr mit dem Inkrafttreten des Bundesbankgesetzes (BBG) die Deutsche Bundesbank gegründet wurde. 
gekennzeichnet war. Die Betrachtung erfolgt vom Zusammenbruch des Bretton Woods-Systems 1971 bis zum Entstehen eines neuen „Festkurssystems“, des EWS, im Jahr 1979. Diese Periode dauerte also von 1972 bis 1978. Da nicht alle Länder von Beginn an am Wechselkursmechanismus teilnahmen, werden zusätzlich in den Spalten 6 und 7 die Werte berücksichtigt, die tatsächlich in die Zeit flexibler Wechselkurse bzw. in der Zeit der EWS-Teilnahme auftraten. Zudem wird berücksichtigt, daß Großbritannien und Italien seit 1992 nicht mehr am EWS teilnehmen und ihre Geldpolitik wieder autonom bestimmen (siehe den Klammerausdruck in Spalte 7). ${ }^{2}$

Auch dieser Vergleich fällt zugunsten Deutschlands aus. Wieder hat die Bundesbank die mit Abstand geringsten Inflationsraten zugelassen. Auffällig ist auch, daß bei allen Partnern - mit Ausnahme Griechenlands - der Abstand zur deutschen Inflationsrate größer wurde. Beispielhaft sei hier auf Portugal als Land mit der höchsten Inflationsrate sowie Großbritannien hingewiesen, die im Durchschnitt von 1957 bis 1995 noch Inflationsdifferenzen von 7,8 bzw. 3,6 Prozentpunkte zu Deutschland realisierten, im Zeitraum der flexiblen Wechselkurse aber 14,1 bzw. 8,6 Prozentpunkte über der deutschen Rate lagen. Zur Zeit des Bretton Woods-Systems betrugen die Differenzen lediglich 1,2 bzw. 1,3 Prozentpunkte. Demnach müßte die Bundesbank auch nach diesem Kriterium die höchste Reputation erzielt haben. ${ }^{3}$ Anhand von Spalte 5 läßt sich zudem erkennen, daß das Schwankungsintervall der Inflationsraten zur Zeit des EWS wieder geringer geworden ist. Läßt man Griechenland als Nichtmitglied des EWS außer Betracht und berücksichtigt, daß Portugal erst 1992 dem EWS beigetreten ist, muß Italien als „,schlechtestes“ Mitglied zum Vergleich herangezogen werden: Es hat mit 9,3\% den höchsten Abstand zum „besten“ Land (Niederlande) mit 2,9\% realisiert. ${ }^{4}$ Die Schwankungsbreite verringerte sich also im neuen „Festkurssystem“ von 14,1 Prozentpunkten zur Zeit der autono-

2 Diese zusätzlichen Werte werden hier nur der Vollständigkeit halber berücksichtigt. An den generellen Aussagen ändern sie jedoch nichts. Zudem können die Werte der drei neuen Partner Finnland, Schweden und Österreich in Spalte 7 noch nicht als repräsentativ betrachtet werden, da sie lediglich das Ergebnis eines Jahres widerspiegeln.

3 Wenn die absoluten Werte auch nicht als Stabilität interpretiert werden können, ist doch die Relation zu den Partnern interessant. Man muß berücksichtigen, daß in diese Zeit die erste Ölpreiskrise fällt.

4 Deutschland realisierte in diesem Zeitraum mit einer durchschnittlichen Inflationsrate von 3,1\% nur noch die zweitbeste Rate. Die Ursache hierfur ist aber nicht in der Geldpolitik der Zentralbank zu sehen, sondern vor allem im „Vereinigungsschock“, der sich seit 1991 in den Inflationsraten widerspiegelt. Vgl. auch Herr/Westphal (1991), S. 82. 
Tabelle 4.1: Durchschnittliche Inflationsraten der EU-Mitglieder ${ }^{5}$

\begin{tabular}{|c|c|c|c|c|c|c|}
\hline Länder & $\begin{array}{c}1957- \\
1995 \\
\end{array}$ & $\begin{array}{l}1957- \\
1971\end{array}$ & $\begin{array}{l}1972- \\
1978\end{array}$ & $\begin{array}{c}1979- \\
1995\end{array}$ & $\begin{array}{c}1972- \\
\text { EWS-Beitritt }\end{array}$ & $\begin{array}{c}\text { seit EWS- } \\
\text { Beitritt }\end{array}$ \\
\hline Belgien & 4,3 & 2,7 & 8,3 & 4,0 & & \\
\hline Dänemark & 5,9 & 4,7 & 10,1 & 5,3 & & \\
\hline Deutschland & 3,2 & 2,5 & 5,1 & 3,1 & & \\
\hline Finnland $^{4)}$ & 6,7 & 5,2 & 12,4 & 5,7 & 7,9 & 1,0 \\
\hline Frankreich & 6,0 & 4,6 & 9,6 & 5,7 & & \\
\hline (Griechenland $^{6)}$ & 10,5 & 2,1 & 13,8 & 17,8 & 16,6 & \\
\hline Großbritannien $^{2)}$ & 6,8 & 3,8 & 13,7 & 6,7 & 10,1 & $6,3(2,5)$ \\
\hline Irland & 7,2 & 4,3 & 13,8 & 7,0 & & \\
\hline Italien $^{5)}$ & 7,7 & 3,2 & 14,2 & 9,3 & & \begin{tabular}{|l}
$10,3(4,6)$ \\
\end{tabular} \\
\hline Luxemburg & 4,0 & 2,4 & 7,3 & 4,1 & & \\
\hline Niederlande & 4,2 & 4,1 & 7,9 & 2,9 & & \\
\hline Österreich $^{4)}$ & 4,0 & 3,2 & 6,9 & 3,6 & 4,6 & 2,3 \\
\hline Portugal $^{3)}$ & 11,0 & 3,7 & 19,2 & 14,4 & 17,8 & 6,2 \\
\hline Schweden ${ }^{4)}$ & 6,2 & 4,1 & 9,1 & 6,9 & 7,8 & 2,5 \\
\hline Spanien $^{1)}$ & 9,3 & 6,8 & 16,2 & 8,8 & 13,2 & 5,6 \\
\hline
\end{tabular}

1) EWS-Beitritt 1989: Wert in Spalte 6 entspricht 1972-1988, Wert in Spalte 7 ent-

2) spricht 1989-1995

2) EWS-Beitritt 1990, -Austritt 1992: Wert in Spalte 6 entspricht 1972-1989, Wert in Spalte 7 entspricht 1990-1992, Wert in Klammern 1993-95

3) EWS-Beitritt 1992: Wert in Spalte 6 entspricht 1972-1991, Wert in Spalte 7 entspricht 1992-1995

4) EWS-Beitritt 1995: Wert in Spalte 6 entspricht 1972-1994, Wert in Spalte 7 entspricht 1995

5) EWS-Austritt 1992: Wert in Spalte 6 entspricht 1979-1992, Wert in Klammern 1993-1995

6) kein EWS-Beitritt: Wert in Spalte 6 entspricht 1972-1995

men Geldpolitik auf 7,6 Prozentpunkte zur Zeit des EWS, also um 6,5 Prozentpunkte.

Aber nicht nur die durchschnittliche Inflationsrate ist für die Reputation bedeutsam, sondern auch die Entwicklung der Inflationsrate in der Zeit. Zur Veranschaulichung der Leistung der nationalen Zentralbanken in dieser Hinsicht sollen die folgenden Abbildungen dienen.

5 Die verwendeten Daten stammen vom IMF (1986) und (1995) sowie der OECD (1996). 
Abbildung 4.1: Die Inflationsraten der EU-Länder von 1957 bis 1995
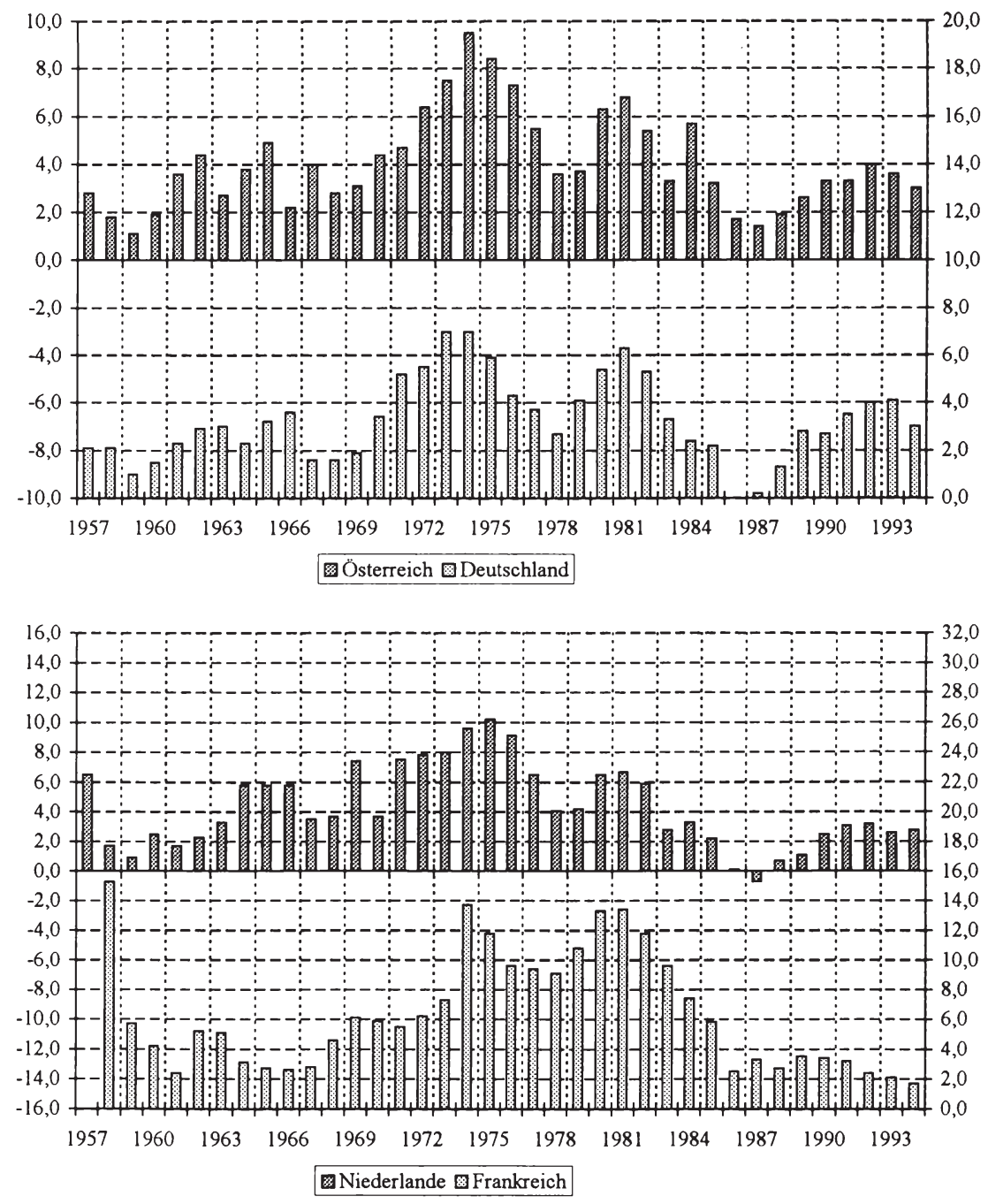

Will man anhand dieser Entwicklungen Aussagen bezüglich des Wunsches bzw. der Realisation des Reputationsaufbaus der nationalen Zentralbanken treffen, gelangt man wieder zum Ergebnis, daß die Bundesbank die höchste Reputation hat, da sie seit ihrem Bestehen kontinuierlich eine Antiinflationspolitik, die zum Teil durch exogene Schocks gestört wurde, verfolgt. Aber 
Abbildung 4.1: $\quad$ Fortsetzung

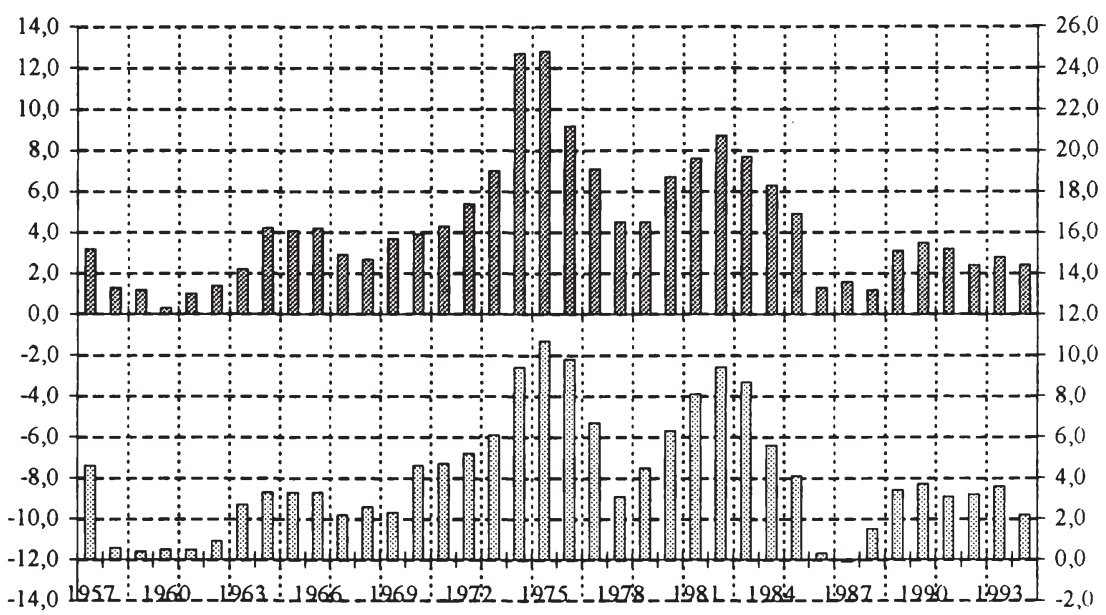

Belgien : Luxemburg

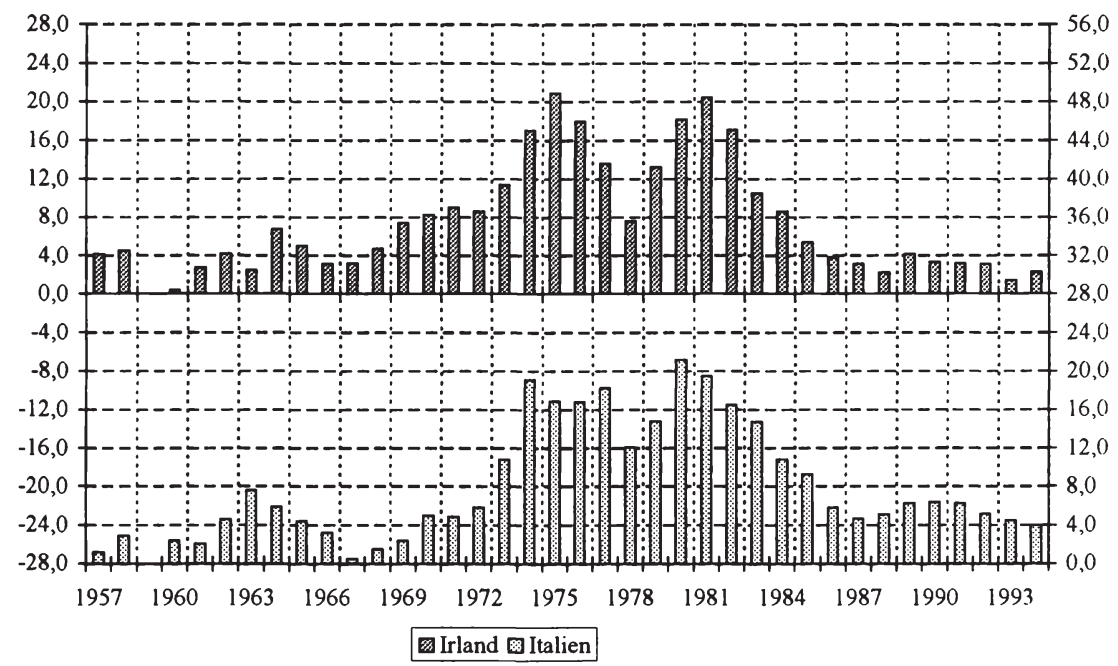

auch Österreich hat seit 1974 beständig versucht, geringe Inflationsraten zu realisieren, die höheren Raten während der beiden Ölpreiskrisen mußte jeder andere Partner auch hinnehmen. Dieser Erfolg Österreichs ist nicht dem EWS zu verdanken, da es erst 1995 beigetreten ist. Allerdings hat die österreichische Zentralbank Glaubwürdigkeit und somit Reputation von Deutschland impor- 


\section{Abbildung 4.1: $\quad$ Fortsetzung}
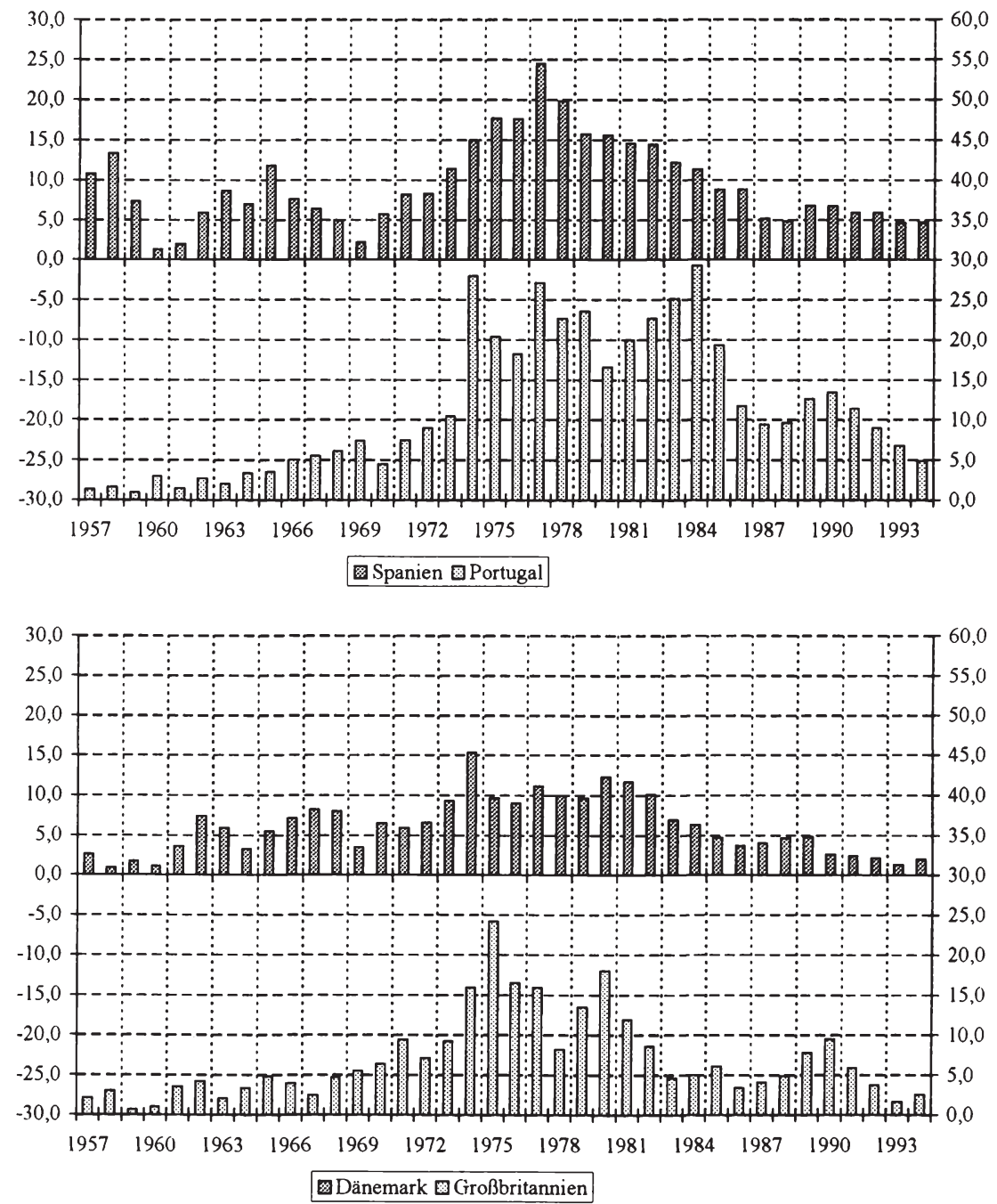

tiert, indem der Schilling fest an die D-Mark gekoppelt wurde, was sich in der ähnlichen Entwicklung der Inflationsrate in Deutschland und Österreich widerspiegelt. 6

6 Das Beispiel Österreich zeigt auch, daß der Wille der Regierung für den Stabilitätsimport wichtiger ist als feste Wechselkurse. Vgl. hierzu Schäfer (1993), S. 102. Öster- 


\section{Abbildung 4.1: $\quad$ Fortsetzung}
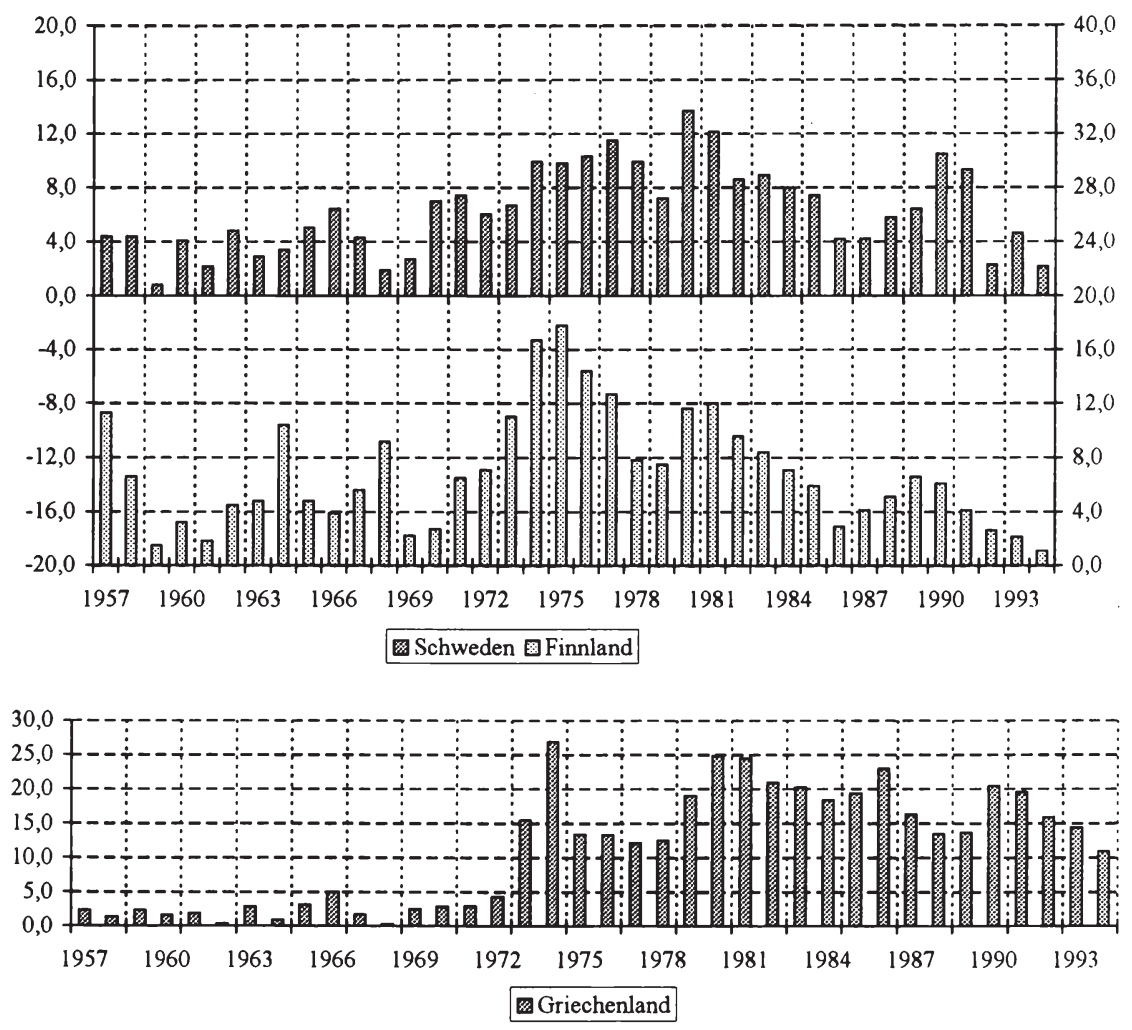

$\mathrm{Zu}$ diesen Niedriginflationsländern können auch die Niederlande gezählt werden, die bereits seit 13 Jahren eine strikte Antiinflationspolitik verfolgen. Auch ihnen sollte es gelungen sein, sich eine gewisse Reputation aufzubauen. Allerdings ist hierbei zu berücksichtigen, daß die Niederlande die deutsche „Vor-

reich ging nach dem Zusammenbruch des Bretton Woods-Systems zunächst zu einer Bindung des Schilling an einen Währungskorb über, der die D-Mark „nur“ zu ca. 50\% gewichtete. Als die anderen Währungen aber abwerteten, wurden sie nacheinander aus dem Korb herausgenommen. Übrig blieb die D-Mark als wertbeständigste Währung, weshalb zwischen Österreich und Deutschland bereits seit 1982 eine De-factoWährungsunion besteht. Zur ausführlichen Diskussion der deutsch-österreichischen „Währungsunion“vgl. Socher (1991). 
herrschaft" im EWS akzeptierten und es als erste Aufgabe ihrer Geldpolitik ansahen, den Wechselkurs zur D-Mark aufrechtzuerhalten. ${ }^{7}$

Eine zweite Ländergruppe bilden Frankreich, Belgien und Luxemburg. Speziell in Frankreich fand 1983 eine wirtschaftspolitische Wende statt, indem nun eine Strategie des „Franc fort" betrieben wurde. Die Rolle der D-Mark als Leitwährung wurde akzeptiert, und zur Stabilisierung der eigenen Währung folgte man der deutschen Geldpolitik. Das Ergebnis zeigte sich ab 1986 in einer gestiegenen Wechselkursstabilität und abnehmenden Inflationsdifferenzen zu Deutschland. Das gestiegene Vertrauen der Märkte spiegelt sich in gesunkenen Zinsdifferenzen wider. ${ }^{8}$ Dieser klare Wechsel zur Antiinflationspolitik ist auch für Belgien und Luxemburg zu erkennen. Zwar realisierten beide auch vorher Inflationsraten, die sich abgesehen von den beiden Ölpreiskrisen um 4\% bewegten, dies aber im Festkurssystem von Bretton Woods. Seit 1986 betragen die Raten in den drei Ländern etwa 3\%. Die zehn Jahre der Inflationsbekämpfung haben sicherlich zum Reputationsaufbau der nationalen Zentralbanken dieser drei Länder geführt. Allerdings wird sich noch zeigen müssen, ob diese Reputation verdient bzw. gefestigt oder nur von der Bundesbank „geliehen“ ist, weil diese aufgrund der von den Gründern nicht beabsichtigten asymmetrischen Funktionsweise des EWS de facto die Geldpolitik in Europa bestimmte. Die Geldwertsicherungspolitik dieser Länder stellte somit keine originäre, sondern eine aus der D-Mark-Ankerfunktion abgeleitete Politik dar, was bei einem Verlust dieses Ankers zu erheblichen Problemen für die Glaubwürdigkeit und somit letztlich für die Reputation führen kann. ${ }^{9}$

Gleiches gilt im Prinzip auch für Irland, nur daß dieses Land bereits seit 1981 seine Inflation zurückführte. ${ }^{10}$ Auch hier muß aber die Frage gestellt werden,

7 Vgl. Szass (1989) oder Duisenberg (1986).

8 Vgl. Collignon (1991), S. 133, Eijffinger (1991), S. 171 und Gäckle (1992), S. 67f. Der Grund der wirtschaftspolitischen Wende in Frankreich ist in der Erfolglosigkeit der vorher praktizierten nachfragestimulierenden Politik zur Beschleunigung des Wachstums zu sehen. Eine Bewertung dieser Politik nehmen Sachs/Wyplosz (1986) vor.

9 Vgl. Vaubel (1989), S. 276 und Hasse (1993a), S. 132 und Gischer (1993), S. 61.

10 Irland wird in empirischen Untersuchungen immer wieder als eindeutiges Beispiel eines Landes herausgestellt, daß durch die Mitgliedschaft im EWS Glaubwürdigkeit importieren konnte. Nur so konnten dann tatsächlich die geringeren Inflationsraten realisiert werden. Diese Annahme entspricht den Ausfuihrungen dieser Arbeit, da es kaum möglich ist, eine Antiinflationspolitik über einen längeren Zeitraum durchzuhalten, wenn die dafür notwendige Glaubwürdigkeit fehlt. Vgl. auch Kremers (1990), aber auch Dornbusch (1989), der diese These insbesondere fur Irland ablehnt. Die Reputationsgewinne aller EWS-Partner diskutiert von Hagen (1989). Literaturübersich- 
ob dies Ausdruck einer vorhandenen bzw. inzwischen entwickelten Stabilitätskultur ist oder dieser Erfolg wesentlich der Bundesbank zu verdanken ist.

Zwar gingen die Inflationsraten in Italien, Spanien und Portugal während ihrer Mitgliedschaft im EWS ebenfalls (nahezu) fortwährend zurück - was zum Aufbau von Reputation führte -, dennoch konnte keines dieser Länder bisher Inflationsraten von weniger als $4 \%$ realisieren. Damit liegen sie deutlich über dem Durchschnitt der Partnerländer, was zur Folge hat, daß ihre Reputation geringer als die der bisher erwähnten Partner sein muß.

Die Tendenz der Inflationsrate in Dänemark ist seit der Mitgliedschaft im EWS abwärts gerichtet und hat sich seit 1990 bei $2 \%$ stabilisiert. Die Reputation der dänischen Zentralbank ist demnach höher als die der drei eben diskutierten Zentralbanken. Sie müßte aufgrund einer ähnlichen Entwicklung etwa der von Frankreich entsprechen.

Die Situation in Großbritannien und Finnland ist durch zyklische Auf- und Abwärtsentwicklungen gekennzeichnet. In Finnland hat die Inflation mittlerweile zwar mit gut $1 \%$ ein sehr geringes Niveau erreicht, dennoch kann die Reputation der Zentralbank noch nicht sehr hoch sein, da die Wirtschaftssubjekte sich aufgrund der vergangenen Entwicklung kaum ein Bild über den weiteren Verlauf machen können. Noch geringer muß die Reputation in Großbritannien sein, da eine stetige Abwärtsentwicklung nur von 1990 bis 1993 zu erkennen ist und seit 1994 schon wieder höhere Raten realisiert werden. ${ }^{11} \mathrm{Zu}$ berücksichtigen ist des weiteren, daß das Pfund seit 1992 nicht mehr am Wechselkursmechanismus teilnimmt.

Eine bestenfalls geringe Reputation können auch Griechenland und Schweden erst besitzen. Zwar ist das Inflationsniveau in Schweden seit den letzten vier Jahren gering, die Entwicklung bis dahin ist aber recht unstetig. Aussagen über die weitere Entwicklung können nicht getroffen werden. In Griechenland ist zwar seit 1990 ein klarer Abwärtstrend zu erkennen, aber das Niveau ist zu hoch, als daß die Zentralbank als Inflationsgegner gelten könnte.

ten zur Diskussion der „EWS-Hypothese“ geben von Hagen (1989) und Weber (1991). Letzterer versucht auch eine Messung der absoluten Reputation der einzelnen EWS-Mitglieder.

11 In Großbritannien ist die Situation gegeben, daß die Wirtschaftssubjekte der Zentralbankspitze zwar den Wunsch nach Reputation zutrauen, dieser aber aufgrund der gegebenen Abhängigkeit von der Regierung nicht realisiert werden kann. 
Es bleibt festzuhalten, daß allein aufgrund der erbrachten Leistung die Bundesbank die höchste Reputation genießt. Dies Ergebnis kann allerdings nicht überraschen, da sie die größte Unabhängigkeit besaß. Die Geldpolitik aller anderen Länder wird mehr oder weniger direkt von der Regierung bestimmt. ${ }^{12}$ Wie im theoretischen Teil gezeigt wurde, unterliegt diese aber aufgrund der Wahlzyklen erheblichen Inflationsanreizen. Insbesondere die kurzfristige Beschäftigungsankurbelung kurz vor Wahlterminen stellt hier ein Hindernis für eine stetige Antiinflationspolitik dar.

\subsection{Die Glaubwürdigkeit einer europäischen Geldpolitik}

Wie modelltheoretisch gezeigt wurde, stellt eine glaubwürdige Geldpolitik eine wesentliche Bedingung für den Reputationsaufbau einer Zentralbank dar. Nur wenn die Wirtschaftssubjekte an eine angekündigte Antiinflationspolitik glauben, kann diese auch ohne Stabilisierungskrise realisiert werden. Haben die Wirtschaftssubjekte jedoch aufgrund einer geringen Reputation der Zentralbank kein Vertrauen in eine Antiinflationspolitik und setzen ihre Erwartungen entsprechend höher als die von der Zentralbank gemachte Ankündigung, kann die Zentralbank nur Reputation aufbauen, indem sie die Erwartungen über eine Stabilisierungskrise „bricht“. Allerdings ist es für die Zentralbank bei höheren Erwartungen nicht mehr optimal, ihre angekündigte Inflationsrate zu realisieren, weil jetzt die höheren Erwartungen berücksichtigt werden müssen. Durchgeführt wird eine Rate, die zwischen angekündigter und erwarteter liegt. Je näher sie an der angekündigten liegt, desto höher ist einerseits der Effekt auf die Reputation, andererseits aber auch die resultierende kurzfristige Arbeitslosigkeit. Längerfristig wird der Reputationsaufbau bei unglaubwürdiger Geldpolitik immer schwieriger, da die dafür notwendigen Überraschungsdeflationen längerfristig nicht durchzuhalten sind. Dies gilt insbesondere, wenn wahlabhängige Politiker die Geldpolitik bestimmen.

Aufgrund der wesentlichen Bedeutung der Glaubwürdigkeit für die Reputation wird nun hinterfragt, welche Inflationsrate die Wirtschaftssubjekte von einer europäischen Zentralbank erwarten könnten, welche Politik also glaubwürdig sein könnte. Es werden dabei unterschiedliche Szenarien betrachtet, da die Glaubwürdigkeit insbesondere davon abhängig sein wird, welche Länder an der Währungsunion teilnehmen werden.

12 $\mathrm{Zu}$ einem Vergleich der verschiedenen Zentralbankstatuten vgl. Studt (1993) und Hasse (1989a). 
Zunächst wird eine These über die glaubwürdige Geldpolitik in den einzelnen Nationalstaaten aufgestellt. ${ }^{13}$ Da die EZB sich gleichberechtigt aus allen Partnern zusammensetzen wird, entscheidet die vergangene Geldpolitik der jeweiligen Partner über die Glaubwürdigkeit ihrer Politik. Zur Bestimmung der Glaubwürdigkeit der Politik der einzelnen Zentralbanken werden die letzten drei Realisationen der Geldpolitik berücksichtigt. Es wird entsprechend der folgenden drei Kriterien vorgegangen:

1. Es wird die letzte Realisation erwartet, wenn die Veränderungen der Inflationsrate im Betrachtungszeitraum nicht das gleiche Vorzeichen aufwiesen.

2. Wenn die Veränderungen das gleiche Vorzeichen hatten,

a. wird diese (Auf- oder Abwärts-) Entwicklung berücksichtigt, falls es vor dem Betrachtungszeitraum (d.h. vor 1992, vgl. Abbildung 4.1) bereits Realisationen in der entsprechenden Größenordnung gegeben hat,

b. wird diese Entwicklung nicht berücksichtigt, falls es in der Vergangenheit (siehe oben) keine entsprechenden Realisationen gab:

1. Wenn die vorhandene Reputation der Zentralbank mit der letzten Realisation vereinbar ist, wird diese erwartet.

2. Wenn die letzte(n) Realisation(en) vor dem Hintergrund der Reputation der Zentralbank als zu niedrig angesehen wird (werden), wird die jüngste Realisation innerhalb des Betrachtungszeitraums erwartet, die gerade noch mit der Reputation für vereinbar gehalten wird.

Anhand von Tabelle 4.2 wird ersichtlich, daß die Inflationsrate in Deutschland seit 1993 deutlich verringert wurde. Zwischen 1993 und 1994 fand ein Sprung von 1,1 Prozentpunkten statt, der nächste Sprung ins Jahr 1995 betrug sogar 1,3 Prozentpunkte. Ein Grund für die relativ hohen Sprünge ist im für Deutschland hohen Ausgangsniveau von 1993 zu sehen, welches noch als „Vereinigungslast“ zu interpretieren ist. Aufgrund dieser klaren Antiinflationspolitik und ihrer schon vorhandenen hohen Reputation werden die Wirtschaftssubjekte der Zentralbank auch eine noch geringere Inflationsrate zutrauen, wenn sie diese ankündigt. Es erscheint jedoch wegen des bereits erreichten Niveaus unrealistisch, wieder einen Sprung von über 1 Prozentpunkt anzunehmen. Deshalb wird hier vorsichtig kalkuliert und eine zugetraute Minderung um 0,5 Prozentpunkte zugestanden, was etwa 50\% des geringeren Sprunges ausmacht. Die resultierende Größe von 1,2\% könnte die erwartete Inflationsrate der Wirtschaftssubjekte darstellen, wenn die Bundesbank diesen Wert ankündigt. Die Wirtschaftssubjekte würden diesen Wert jedoch nicht ,automatisch" aufgrund der vergangenen drei Realisationen erwarten, sondern erst dann, wenn die Zentralbank ankündigt, diesen aufgrund ihrer Präferenzen reali- 
Tabelle 4.2: „Glaubwürdigkeit“ der nationalen Geldpolitiken

\begin{tabular}{|l|c|c|c|c|c|}
\hline Land & 1993 & 1994 & 1995 & Entwicklung & $\begin{array}{c}\text { "glaubwürdige“ } \\
\text { Inflationsrate* }\end{array}$ \\
\hline Deutschland & 4,1 & 3,0 & 1,7 & abwärts & 1,2 \\
\hline Österreich & 3,6 & 3,0 & 2,3 & abwärts & 2,0 \\
\hline Niederlande & 2,6 & 2,8 & 2,0 & - & 2,0 \\
\hline Frankreich & 2,1 & 1,7 & 1,7 & - & 1,7 \\
\hline Belgien & 2,8 & 2,4 & 1,4 & abwärts & 1,4 \\
\hline Luxemburg & 3,6 & 2,2 & 2,0 & abwärts & 2,0 \\
\hline Irland & 1,4 & 2,3 & 2,4 & aufwärts & 2,5 \\
\hline Italien & 4,5 & 4,0 & 5,4 & - & 5,4 \\
\hline Spanien & 4,6 & 4,7 & 4,6 & - & 4,6 \\
\hline Portugal & 6,8 & 4,9 & 4,1 & abwärts & 4,1 \\
\hline Dänemark & 1,3 & 2,0 & 2,0 & - & 2,0 \\
\hline Großbritannien & 1,6 & 2,5 & 3,4 & aufwärts & 4,3 \\
\hline Schweden & 4,6 & 2,2 & 2,5 & - & 2,5 \\
\hline Finnland & 2,1 & 1,1 & 1,0 & abwärts & 2,1 \\
\hline Griechenland & 14,4 & 10,9 & 9,3 & abwärts & 9,3 \\
\hline * Diese Inflationsraten ergeben sich bei Anwendung der beschriebenen Kriterien. \\
\hline
\end{tabular}

sieren zu wollen. Dann könnte sie die Inflation bis auf $1,2 \%$ im nächsten Jahr weiter zurückführen, ohne dafür eine Stabilisierungskrise in Kauf nehmen zu müssen.

Ein klarer Abwärtstrend, wenn auch nicht so ausgeprägt, ist in Österreich zu erkennen. Die Verringerungen betrugen hier 0,6 bzw. 0,7 Prozentpunkte. Wieder kann eine Fortführung der strikten Antiinflationspolitik erwartet werden. Aber auch hier wird vorsichtig kalkuliert, indem der nächste Sprung mit 0,3 Prozentpunkten - wieder 50\% des geringeren bereits realisierten Sprunges entsprechend - angesetzt wird. Die geringste Inflationsrate für 1996, die die Wirtschaftssubjekte noch für glaubwürdig halten könnten, beträgt demnach $2 \%$. Sollte die österreichische Zentralbank dann eine geringere Rate realisieren wollen, müßte sie dafür eine Stabilisierungskrise hinnehmen.

Eine andere Entwicklung ist in den Niederlanden zu erkennen. Die Inflationsrate nahm 1994 gegenüber 1993 zu und ging 1995 wieder zurück. Deshalb wird hier davon ausgegangen, daß die Wirtschaftssubjekte eine Ankündigung der Zentralbank in Höhe der letzten Realisation für glaubwürdig halten. Gleiches gilt für Frankreich, da auch hier die vergangene Rate, die sogar in den 
beiden letzten Jahren realisiert wurde, den Wirtschaftssubjekten für das nächste Jahr glaubwürdig erscheinen könnte.

In Belgien und Luxemburg ist wieder ein klarer Abwärtstrend zu erkennen. Da die längerfristige Erfahrung im Gegensatz zu Deutschland und Österreich (vgl. hierzu Abbildung 4.1) aber gegen noch geringere Werte spricht (Kriterium 2b1), könnten die Wirtschaftssubjekte auch hier Inflationsraten in Höhe der letzten Realisation erwarten.

In Irland ist eine klare Aufwärtsentwicklung der Inflationsrate feststellbar, weshalb es durchaus realistisch erscheint, daß die Wirtschaftssubjekte von einer entsprechenden Entwicklung auch für das nächste Jahr ausgehen könnten. Die Differenz wird hier mit 0,1 Prozentpunkten gewählt, da eine solche auch im Jahr davor realisiert wurde. Der Wert für das Jahr 1993 kann für Irland als „Ausreißer“ interpretiert werden, da so ein geringer Betrag im gesamten Betrachtungszeitraum nur einmal, im Jahr 1960, realisiert wurde.

Italien und Spanien weisen keine stetige Entwicklung in den letzten drei Jahren auf, deshalb wird hier unterstellt, daß wieder die letzten Realisationen erwartet werden. In Portugal liegt zwar ein klarer Abwärtstrend vor, eine noch geringere Inflationsrate zu erwarten wäre aber - analog zu Belgien und Luxemburg unrealistisch, da das bereits erreichte Niveau in den letzten zwanzig Jahren nicht unterschritten wurde (Kriterium 2b1).

In Dänemark ist eine ähnliche Entwicklung wie in Frankreich beobachtbar. Auch hier wurden in den letzten beiden Jahren Inflationsraten in gleicher Höhe realisiert, weshalb sie auch für das nächste Jahr glaubwürdig erscheinen könnte.

In Großbritannien liegt eine aufwärts gerichtete Entwicklung vor. Die Differenzen zwischen den Jahresraten betrugen je 0,9 Prozentpunkte, deshalb könnte eine Fortsetzung dieser Entwicklung unterstellt werden.

Für Schweden wird hier die letzte Realisation erwartet, da die letzten drei Jahre keine stetige Entwicklung erkennen lassen (Kriterium 1). In Finnland ist zwar ein klarer Abwärtstrend zu erkennen, dennoch ist es eher unwahrscheinlich, daß die Wirtschaftssubjekte eine solche strikte Antiinflationspolitik schon für glaubwürdig halten könnten. Zudem muß berücksichtigt werden, daß die Entwicklung in Finnland seit 1969 durch ständige Auf- und Abwärtsentwicklungen gekennzeichnet ist. 1975 begann ein vier Jahre und 1981 ein fünf Jahre andauernder Abwärtstrend. Die aktuelle Abwärtsentwicklung hält nun bereits sechs Jahre an, und es erscheint somit plausibel, wenn für das nächste Jahr 
wieder mit einem Anstieg der Inflationsrate gerechnet wird. Zudem ist das bereits erreichte Niveau vorher noch nie erzielt worden (vgl. Abbildung 4.1). Aufgrund der vergangenen Erfahrungen wird deshalb angenommen, da $\beta$ die Wirtschaftssubjekte eine Inflationsrate in Höhe von $2,1 \%$ für realistisch halten könnten, welche auch im kurzfristigen Betrachtungszeitraum realisiert worden ist (Kriterium 2b2). Eine erwartete Inflationsrate von $1 \%$ oder weniger wäre vor dem Hintergrund der bisher aufgebauten Reputation der Zentralbank unrealistisch.

Für Griechenland gilt das gleiche wie für Portugal, weshalb auch hier angenommen wird, daß trotz des klaren Abwärtstrends bestenfalls die jüngste Realisation für glaubwürdig gehalten wird (Kriterium 2b1).

Geht man nun von diesen nationalen „Glaubwürdigkeitswerten“ aus, erhält man erste Anhaltspunkte dafür, welche Antiinflationspolitik einer europäischen Zentralbank gerade noch glaubwürdig sein könnte. ${ }^{14}$ Im Rahmen dieser Arbeit werden zwei Möglichkeiten der Erwartungsbildung als realistisch angenommen. Die erste Alternative besteht darin, daß die Wirtschaftssubjekte die Inflationsrate für glaubwürdig halten könnten, die das „schlechteste“ Mitglied der Gruppe der erfolgreichsten Inflationsbekämpfer realisieren kann. Da im Zentralbankrat Mehrheitsentscheidungen notwendig sind, werden je nach Anzahl der Teilnehmer die Länder mit den geringsten Inflationsraten betrachtet, die zusammen eine Mehrheit bilden. Die höchste dieser Realisationen soll auch jedem erfolgreicheren Partner zugetraut werden. Die zweite Alternative der Erwartungsbildung besteht darin, daß die Wirtschaftssubjekte den Durchschnittswert der beteiligten Mitglieder erwarten.

Tabelle 4.3 zeigt in der vierten Spalte die geringstmögliche glaubwürdige Inflationsrate der EZB, wenn die Mehrheit die Erwartungen bestimmt (erste Alternative) und in der fünften Spalte die Werte, die sich als Durchschnitt der jeweiligen Partner ergeben (zweite Alternative). In der zweiten Spalte werden die jeweiligen Partner der EZB genannt und in der dritten Spalte die Anzahl der Mitglieder sowie die sich daraus ergebende notwendige Mehrheit. Als Grundlage für die Berechnungen dienen die „Glaubwürdigkeitswerte“ aus Tabelle 4.2.

14 Es ist allerdings zu betonen, daß es sich bei dieser Betrachtungsweise um eine bloße Extrapolation der Inflationsraten handelt, d.h. hier wird eine Fortentwicklung der bisherigen Realisationen unterstellt. Tatsächlich stellt die Gründung der EZB einen "qualitativen institutionellen Sprung“ dar, der zu ganz anderen Ergebnissen führen kann. 
Tabelle 4.3: „Glaubwürdige“ Antiinflationspolitik der EZB

\begin{tabular}{|c|l|c|c|c|}
\hline & Mitglieder & $\begin{array}{c}\text { Anzahl } \Rightarrow \\
\text { Mehrheit }\end{array}$ & Mehrheit & $\begin{array}{c}\text { Durch- } \\
\text { schnitt }\end{array}$ \\
\hline 1. & Deutschland + Frankreich & $2 \Rightarrow 2$ & 1,7 & 1,45 \\
\hline 2. & $1 .+$ Belgien & $3 \Rightarrow 2$ & 1,4 & 1,43 \\
\hline 3. & $2 .+$ Luxemburg + Niederlande & $5 \Rightarrow 3$ & 1,7 & 1,66 \\
\hline 4. & $3 .+$ Österreich & $6 \Rightarrow 4$ & 2,0 & 1,72 \\
\hline 5. & $4 .+$ Italien & $7 \Rightarrow 4$ & 2,0 & 2,24 \\
\hline 6. & $5 .+$ Irland & $8 \Rightarrow 5$ & 2,0 & 2,28 \\
\hline 7. & 6. - Italien & $7 \Rightarrow 4$ & 2,0 & 1,80 \\
\hline 8. & $6 .+$ Finnland & $9 \Rightarrow 5$ & 2,0 & 2,26 \\
\hline 9. & 8. - Italien & $8 \Rightarrow 5$ & 2,0 & 1,86 \\
\hline 10. & $8 .+$ Schweden & $10 \Rightarrow 6$ & 2,0 & 2,28 \\
\hline 11. & 10 - Italien & $9 \Rightarrow 5$ & 2,0 & 1,93 \\
\hline 12. & $6 .+$ Spanien & $9 \Rightarrow 5$ & 2,0 & 2,54 \\
\hline 13. & $12 .+$ Portugal & $10 \Rightarrow 6$ & 2,0 & 2,69 \\
\hline 14. & $10 .+$ Spanien & $11 \Rightarrow 6$ & 2,0 & 2,50 \\
\hline 15. & $14 .+$ Portugal & $12 \Rightarrow 7$ & 2,1 & 2,60 \\
\hline 16. & $4 .+$ Dänemark & $7 \Rightarrow 4$ & 2,0 & 1,76 \\
\hline 17. & $5 .+$ Dänemark & $8 \Rightarrow 5$ & 2,0 & 2,21 \\
\hline 18. & $6 .+$ Dänemark & $9 \Rightarrow 5$ & 2,0 & 2,25 \\
\hline 19. & $7 .+$ Dänemark & $8 \Rightarrow 5$ & 2,0 & 1,83 \\
\hline 20. & $8 .+$ Dänemark & $10 \Rightarrow 6$ & 2,0 & 2,23 \\
\hline 21. & $10 .+$ Dänemark & $11 \Rightarrow 6$ & 2,0 & 2,26 \\
\hline 22. & $12 .+$ Dänemark & $10 \Rightarrow 6$ & 2,0 & 2,49 \\
\hline 23. & $13 .+$ Dänemark & $11 \Rightarrow 6$ & 2,0 & 2,63 \\
\hline 24. & $14 .+$ Dänemark & $12 \Rightarrow 7$ & 2,0 & 2,46 \\
\hline 25. & $15 .+$ Dänemark & $13 \Rightarrow 7$ & 2,0 & 2,55 \\
\hline 26. & $4 .+$ Irland + Finnland + Dänemark & $9 \Rightarrow 5$ & 2,0 & 1,88 \\
\hline 27. & $25 .+$ Großbritannien & $14 \Rightarrow 8$ & 2,1 & 2,68 \\
\hline 28. & $27 .+$ Griechenland & $15 \Rightarrow 8$ & 2,1 & 3,12 \\
\hline & & & & \\
\hline & & & \\
\hline
\end{tabular}

Es werden insgesamt 28 unterschiedliche Szenarien betrachtet. Die erste Möglichkeit besteht darin, daß zunächst nur die beiden „großen“ Partner Deutschland und Frankreich eine Währungsunion eingehen. Da für Belgien mit 1,4\% die zweitgeringste Inflationsrate als glaubwürdig unterstellt wurde, wird auch die Alternative einer EZB bestehend aus diesen drei Partnern durchdacht. Dann 
wird der Fall betrachtet, daß Deutschland, Frankreich und die Benelux-Länder die Währungsunion gründen. Im vierten Fall wird zusätzlich das neue Mitglied Österreich berücksichtigt. Danach setzt sich die EZB neben den bisherigen Mitgliedern auch aus den Gründungsmitgliedern des EWS Italien und Italien/ Irland bzw. im siebten Fall ohne Italien zusammen. Die nächsten vier Fälle beziehen auch die beiden neuen Mitglieder Finnland und Schweden in die Betrachtung mit ein, je mit und ohne Italien. Dann werden die Partner Spanien und Portugal berücksichtigt. Denkbar sind Lösungen mit und ohne die neuen Mitglieder. Die Fälle 16 bis 26 berücksichtigen einen Beitritt Dänemarks. Dieses Land hat sich allerdings genau wie Großbritannien eine „Opting out“-Klausel für die Währungsunion vorbehalten. Aufgrund der relativ geringen Inflationsrate, die in Dänemark glaubwürdig erscheint, werden verschiedene Szenarien betrachtet. So besteht die Währungsunion zunächst aus den Partnern Deutschland, Frankreich, den Benelux-Ländern, Österreich sowie Dänemark. Dann kommen nacheinander noch Italien, Irland, Finnland, Schweden, Spanien und Portugal hinzu. Auch die Möglichkeit, daß Italien sowie die beiden neuen Mitglieder nicht sofort teilnehmen, wird berücksichtigt. Der Vollständigkeit halber werden abschließend die beiden weniger realistischen Fälle berücksichtigt, daß Großbritannien bzw. Großbritannien und Griechenland von Beginn an Teilnehmer der Währungsunion sind.

Anhand der unterschiedlichen Szenarien läßt sich ablesen, daß für die Wirtschaftssubjekte eine Inflationsrate von weniger als $2 \%$ (nach der ersten Methode der Erwartungsbildung) vermutlich nur glaubwürdig ist, wenn die Währungsunion von Deutschland, Frankreich und den Benelux-Ländern gebildet wird. Allerdings ergibt sich das gleiche Ergebnis, wenn statt Luxemburg oder den Niederlanden Österreich als Mitglied hinzugenommen wird, da für diese Länder mit $2 \%$ die gleiche Inflationsrate glaubwürdig erscheint. Nimmt man aber alle drei in die Währungsunion auf, ergibt sich wegen der dann notwendigen Mehrheit von vier Stimmen ,nur" noch eine glaubwürdige Geldpolitik von $2 \%$. Diese wird in nahezu allen Konstellationen erwartet. Nur für den Fall, daß Großbritannien oder Großbritannien und Griechenland beitreten, steigt die erwartete Rate auf 2,1\% an. Gleiches gilt für den fünfzehnten Fall, in dem bis auf Dänemark, Großbritannien und Griechenland alle Partner des EWS auch an der Währungsunion teilnehmen.

Realistischer als diese Erwartungsbildung dürfte aufgrund der differenzierteren Ergebnisse jedoch die zweite Methode sein. Hiernach wird eine Inflationsrate von unter $2 \%$ nur dann für glaubwürdig gehalten, wenn Italien kein Mitglied der EZB ist. Dies erscheint aufgrund der in Italien bisher realisierten Politik und demzufolge überdurchschnittlich hohen Inflationserwartungen von 5,4\% 
realistisch. Die beste Alternative stellt auch hier eine Währungsunion zwischen Deutschland, Frankreich und Belgien dar.

Stellt man eine Rangfolge auf, nach der die Partner der EZB beitreten dürften, müßte diese entsprechend den nationalen „Glaubwürdigkeitswerten“ gebildet werden. Tabelle 4.4 zeigt das Ergebnis.

Tabelle 4.4: Rangfolge des Beitritts der EU-Länder zur Währungsunion entsprechend ihrer nationalen "Glaubwürdigkeit“

\begin{tabular}{|c|l|c|c|}
\hline & Land & $\begin{array}{c}\text { nationale } \\
\text { „Glaubwürdigkeit“ }\end{array}$ & Durchschnitt \\
\hline 1. & Deutschland & 1,2 & \\
\hline 2. & Belgien & 1,4 & 1,30 \\
\hline 3. & Frankreich & 1,7 & 1,40 \\
\hline 4. & Luxemburg & 2,0 & 1,58 \\
\hline 4. & Niederlande & 2,0 & 1,66 \\
\hline 4. & Österreich & 2,0 & 1,70 \\
\hline 4. & Dänemark & 2,0 & 1,76 \\
\hline 8. & Finnland & 2,1 & 1,80 \\
\hline 9. & Irland & 2,5 & 1,88 \\
\hline 9. & Schweden & 2,5 & 1,94 \\
\hline 11. & Portugal & 4,1 & 2,14 \\
\hline 12. & Großbritannien & 4,3 & 2,32 \\
\hline 13. & Spanien & 4,6 & 2,50 \\
\hline 14. & Italien & 5,4 & 2,70 \\
\hline 15. & Griechenland & 9,3 & 3,14 \\
\hline
\end{tabular}

Je mehr Mitglieder entsprechend dieser Reihenfolge beitreten, desto höher würde die für glaubwürdig gehaltene Inflationsrate (Spalte 4). $\mathrm{Zu}$ berücksichtigen ist, daß aufgrund gleicher "Glaubwürdigkeitswerte" die Reihenfolge des Beitritts von Luxemburg, den Niederlanden, Österreich und Dänemark den Durchschnittswert nicht verändert, der Durchschnitt aber zwangsläufig steigt, je mehr von ihnen beitreten. Gleiches gilt für Irland und Schweden. Interessant ist auch die Rangfolge Italiens, das der Währungsunion vom Beginn an angehören möchte. Auf kürzere Sicht ist Italiens Beitritt ökonomisch aber nicht zu rechtfertigen, da dies nur um so größere Verluste für die stabilitätsbewußteren Partner bedeuten würde. Als Ergebnis läßt sich festhalten, daß jedes Land, welches glaubwürdig eine geringere Inflationsrate bei nationaler Geldpolitik realisieren könnte, durch die Währungsunion Verluste hinnehmen muß, wäh- 
rend jedes beteiligte „Hochinflationsland“ gewinnt. 15 In der ersten Gruppe ist eine vorher glaubwürdige Geldpolitik nicht mehr durchführbar, ohne nun eine Stabilisierungskrise hinzunehmen, während in den anderen Ländern sprungartig eine geringere Inflationsrate glaubwürdig wird.

Bei allen bisherigen Ergebnissen ist jedoch zu berücksichtigen, daß die Überlegungen nur auf Grundlage bereits vergangener Realisationen bei gegebenen Rahmenbedingungen angestellt werden konnten. Die Gründung der EZB stellt aber eine neue Ausgangssituation dar, weil der institutionelle Rahmen wesentlich verändert wird. Dieser Punkt wird in der Diskussion um die Währungsunion allerdings ständig vernachlässigt. Seit Bestehen des EWS wurde insofern eine Wettbewerbslösung praktiziert, als die nationalen Zentralbanken miteinander konkurrierten und die erfolgreichste automatisch eine „Führerrolle“ übernahm. In diesem Lichte könnte auch die Annäherung der anderen Partner an die geringen Inflationsraten der Bundesbank gesehen werden. Nun aber soll die Wettbewerbslösung zugunsten der Verhandlungslösung aufgegeben werden. ${ }^{16}$ Die Zentralbanken konkurrieren dann nicht mehr untereinander, sondern müssen sich in Verhandlungen über eine bestimmte Geldpolitik einigen. Jeder Partner darf dann entsprechend seinen tatsächlichen Präferenzen für eine bestimmte Inflationsrate stimmen, ohne daß er dabei durch andere Partner restringiert wird. Es könnte demnach der Fall eintreten, daß sich die derzeit ,guten“ Ergebnisse der Partnerländer bei einem anderen institutionellen Rahmen ins Gegenteil verkehren, wenn sich noch keine entsprechende Stabilitätskultur herausgebildet haben sollte.

Ein besonderes Problem für die Glaubwürdigkeit stellt die Tatsache dar, daß innerhalb der Währungsunion kein Anreiz zur Senkung der Staatsverschuldung besteht, sondern eher im Gegenteil ein Anreiz zu deren Steigerung, da sich im gemeinsamen Währungsgebiet ein einheitlicher Zinssatz herausbilden wird. Die sich verschuldenden Länder müssen dann eine geringere Zinssteigerung hinnehmen, als wenn sie ihre Geldpolitik weiterhin in nationaler Verantwortung beließen, da der Effekt nun ein größeres Währungsgebiet betrifft. Die durch nur einige Partnerländer verursachten Zinssteigerungen stellen negative externe Effekte dar, die von Dritten zu tragen sind. Für die Wirtschaftssubjekte und Unternehmen der stabilitätsbewußteren Partnerländer können die Zinssteige-

15 Der „größte“ Verlierer der Währungsunion wäre demnach Deutschland, da die Bundesbank die erfolgreichste Zentralbank ist. Vgl. auch Herr/Westphal (1991), S. 100 und Socher (1991), S. 388. 
rungen eine abnehmende Investitionstätigkeit und eine steigende Staatsverschuldung implizieren, die eventuell zu steigenden Steuerbelastungen führt. ${ }^{17}$

Die betrachteten Szenarien stellen also nur die günstigsten Bedingungen dar, daß die Partner ihre (mehr oder weniger) strenge Antiinflationspolitik auch beibehalten. Dies ist aber, wie ausgeführt wurde, in keiner Weise gewährleistet. Um präzisere Aussagen über die Reputation der EZB machen zu können, muß also der geplante institutionelle Rahmen genauer untersucht werden, da dieser ausschlaggebend ist.

\subsection{Die Unabhängigkeit der EZB}

Im theoretischen Teil dieser Arbeit hat sich die Unabhängigkeit einer Zentralbank als notwendige Bedingung zum Reputationsaufbau erwiesen. Aufgrund der positiven empirischen Erfahrungen haben auch die verantwortlichen Politiker im Vertrag über die Europäische Union (Maastricht-Vertrag), der die Gründung einer europäischen Zentralbank vorsieht, sowie in der Satzung der EZB deren Unabhängigkeit festgeschrieben. ${ }^{18}$

Da die Bundesbank die unabhängigste Zentralbank Europas ist und zudem die höchste Reputation genießt, diente das Bundesbankgesetz (BBG) als Grundlage für die Satzung der EZB bzw. der einschlägigen Artikel im MaastrichtVertrag, um so auch die EZB mit einer möglichst hohen Anfangsreputation auszustatten. Um die Ähnlichkeit mit dem BBG aufzuzeigen, werden neben den Regelungen für die EZB auch die Bestimmungen für die Bundesbank herausgestellt.

Gemäß Artikel 107 des EU-Vertrags bzw. Artikel 7 der Satzung dürfen weder die EZB noch eine nationale Zentralbank bei der Durchführung ihrer Aufgaben Weisungen von Gemeinschaftsorganen oder nationalen Regierungen einholen oder entgegennehmen. Entsprechend verpflichten sich die Gemeinschaftsorgane bzw. die nationalen Regierungen, gemäß diesem Grundsatz zu handeln und eine Beeinflussung der EZB oder ihrer Mitglieder zu unterlassen. Diese Forderung entspricht im wesentlichen dem $\$ 12$ Satz 2 im BBG. Wie konkret diese Unabhängigkeit aber im Gesetz festgelegt ist bzw. welche zur Reputationssteigerung aufgestellten Kriterien sie erfüllt, soll nun ausführlicher untersucht werden.

17 Vgl. Berthold (1992), Herr/Westphal (1991), S.89ff. und Vaubel (1993), S. 60f.

18 Der Maastricht-Vertrag folgt im wesentlichen den Vorschlägen des Delors-Berichts, der hier nicht mehr explizit herausgestellt wird. Vgl. Delors (1989). 
Damit die Zentralbank in funktioneller Hinsicht als unabhängig gelten kann, muß ihre Unabhängigkeit eindeutig im Gesetz festgelegt sein, welches dann Verfassungsrang erhält bzw. nur mit qualifizierter Mehrheit zu ändern ist. Im Gegensatz zum BBG, in dem zwar die Unabhängigkeit in $\$ 12$ festgelegt ist, deren Aufhebung aber nur eine einfache Mehrheit des Parlaments benötigt, ist die Unabhängigkeit der EZB nicht nur in deren Satzung festgelegt, sondern auch im Vertrag über die Europäische Union, der nur einstimmig von allen Regierungschefs der Mitglieder der EU-Länder geändert werden kann, da er einen völkerrechtlichen Vertrag darstellt. Zusätzlich ist noch die Ratifikation durch die nationalen Parlamente erforderlich, womit die Unabhängigkeit quasi einen verfassungsrechtlichen Rang erhält. Die Unabhängigkeit der EZB kann somit zunächst als gesicherter als die der Bundesbank betrachtet werden. ${ }^{19}$

Die Forderung nach einer eindeutigen und einzigen Zielvorgabe der Preisniveaustabilisierung für die Zentralbank wurde genau wie im BBG nur teilweise erfüllt. Zwar geben auch der EU-Vertrag in den Artikeln 3a II und 105 II sowie die Satzung mit Artikel 2 die Preisniveaustabilisierung als vorrangiges Ziel der EZB an ${ }^{20}$, in denselben Artikeln wird aber zusätzlich unter Wahrung ihrer primären Aufgabe die Unterstützung der allgemeinen Wirtschaftspolitik gefordert, damit die umfangreichen Ziele des Artikel 2 des EU-Vertrags erfüllt werden können. Die Ziele der europäischen Wirtschaftspolitik gehen dabei über die vier Ziele der deutschen Wirtschaftspolitik hinaus, wie sie im Stabilitätsgesetz verankert sind und deren Erreichung die Bundesbank unter Wahrung ihrer Aufgabe zu unterstützen hat (Artikel 12 Satz 1 BBG). Zudem wird die Unterstützung der allgemeinen Wirtschaftspolitik auf europäischer Ebene in den gleichen Artikeln genannt, in denen auch das primäre Ziel aufgeführt ist. Im BBG wird diese allgemeine Pflicht erst an späterer Stelle (\$12) genannt, während ihre primäre Aufgabe in $\S 3$ aufgeführt ist. Diese Erwähnung der sekundären Aufgabe in einem separaten, nachgelagerten Artikel betont zugleich die größere Bedeutung der eigentlichen Aufgabe.

Dennoch erfüllt keine der beiden Rechtsgrundlagen für die Zentralbanken die für die Reputation aufgestellte Forderung nach der Ausschließlichkeit der Nennung der Preisniveaustabilisierung. Auf beide Zentralbanken ist somit ein politischer Druck, auch andere Ziele mit Hilfe der Geldpolitik zu begünstigen, nicht auszuschließen. Allein die Existenz einer solchen Möglichkeit kann schon

19 Vgl. Bofinger (1993), S. 44, Hasse (1989a), S. 156 und Schlüter (1991), S. 109.

20 Wörtlich wird die Preisniveaustabilität nur von der EZB verlangt, die Bundesbank hat den etwas „allgemeineren“ Auftrag, die Währung zu sichern. Da letzteres aber nur über die Erfüllung der Preisniveaustabilisierung zu gewährleisten ist, wird dieser Unterschied hier nicht weiter ausgeführt. 
reputationsmindernd wirken. Es kann zwar argumentiert werden, daß dieser Druck auf die EZB erheblich geringer ausfält als auf eine nationale Zentralbank, da jede nationale Regierung nur einen Zentralbankpräsidenten entsendet, auf den sie Druck ausüben kann, und somit muß schon eine Mehrheit Druck ausübender nationaler Regierungen existieren. Dennoch hätte der Verzicht auf eine solche „Zusatzaufgabe“ reputationssteigernd wirken können, da die alleinige Nennung der Preisniveaustabilisierung ein sichtbares Zeichen für alle Wirtschaftssubjekte hätte sein können, daß es der EZB primär bzw. ausschließlich um ihre Reputationssteigerung geht. Es ist auch kaum anzunehmen, daß die EZB ohne diese zusätzliche Zielvorgabe bewußt zu Lasten anderer Ziele agiert, wenn dies zur Erreichung ihres primären Ziels nicht erforderlich ist. Ein Verzicht auf diese Klausel hätte also keine negativen Konsequenzen, könnte andererseits aber durch seine Signalwirkung reputationssteigernd gewirkt haben.

Auf direkte Einwirkungsrechte von Gemeinschaftsorganen oder der nationalen Regierungen wurde im EZB-Statut im Gegensatz zum BBG verzichtet. Letzteres sieht für die Regierung ein aufschiebendes Vetorecht insofern vor, als geldpolitische Entscheidungen um vierzehn Tage verzögert werden können $(\$ 13$ II). Ein solches Recht haben die europäischen Parlamentarier im Franz-Bericht (Artikel 7 IV) für sich zwar auch gefordert ${ }^{21}$, letztlich fand dies aber keinen Niederschlag in der Satzung der EZB. Der Präsident des Rats und ein Mitglied der Kommission können an den Sitzungen des EZB-Rats teilnehmen, haben aber kein Stimmrecht (Artikel 109b I des Vertrags). Der Präsident kann lediglich Anträge zur Beratung vorlegen.

Nach Artikel 15 III der Satzung bzw. Artikel 109b III des Maastricht-Vertrags unterbreitet die EZB dem Europäischen Parlament, dem Rat und der Kommission einen Jahresbericht über ihre vergangene und zukünftige Tätigkeit. Hinzu kommen vierteljährliche Berichte über ihre Tätigkeit sowie wöchentlich ein konsolidierter Ausweis, welche zu veröffentlichen sind. Diese Berichtspflichten können eher reputationssteigernd wirken, da so der „Mythologisierung“ der Geldpolitik entgegengewirkt wird.

Damit die funktionelle Unabhängigkeit faktisch aber realisierbar ist, muß auch die instrumentelle Unabhängigkeit gegeben sein. Diese sieht ein ausreichendes und wirksames Instrumentarium für die Zentralbank vor, über welches sie die volle Kontrolle besitzt. Zudem darf keine direkte Kreditvergabe an die Regierung vorgesehen sein, und die Kompetenz für die Geld- und Währungspolitik

Vgl. Franz (1990), S. 360f. 
muß in einer Hand liegen, d.h. die Zentralbank muß auch die Entscheidungskompetenz über die Wechselkurspolitik besitzen. ${ }^{22}$ Die Instrumentenfrage ist in den Artikeln 18 bis 20 der Satzung geregelt. Neben den marktkonformen Instrumenten der Offenmarkt- und Kreditgeschäfte besteht für die EZB genau wie für die Bundesbank die Möglichkeit, eine Mindestreserve zu erheben (Artikel 19 bzw. §16). Eine solche Zwangsmaßnahme wurde als der Reputation förderlich angenommen, weil so das technologische Glaubwürdigkeitsproblem zumindest gemindert werden könnte. Wie beschrieben, kann bereits allein die Existenz dieses Instrumentes disziplinierend auf die Übermittler der monetären Impulse wirken.

Die Forderung nach dem Verbot der direkten Kreditvergabe an öffentliche Stellen erfüllt die Satzung der EZB ebenso wie das BBG. ${ }^{23}$ Gemäß Artikel 104 I des Maastricht-Vertrags bzw. Artikel 21 I der Satzung besteht ein absolutes Verbot der Kreditvergabe sowie des unmittelbaren Erwerbs von Schuldtiteln nationaler Regierungen durch die EZB.

Eine empfindliche Störung der instrumentellen Unabhängigkeit ist aber durch die fehlende Wechselkurskompetenz der EZB gegeben. Wie im BBG bleibt auch in der Satzung der EZB die Währungspolitik unberücksichtigt. Der Maastricht-Vertrag ordnet die Währungspolitik in Artikel 109 klar den Politikern zu, die EZB wird lediglich dazu gehört. Zwar muß die Wechselkurspolitik mit dem primären Ziel der Preisniveaustabilität der EZB im Einklang stehen, welche Politik diese Forderung jedoch erfült, beurteilt nicht ein Gremium der EZB, sondern bleibt den Politikern überlassen. Diese Regelung kann dazu beitragen, daß die Wirtschaftssubjekte der EZB nicht die optimale Reputation zuerkennen, selbst wenn sie davon überzeugt sind, daß der Reputationsaufbau das primäre Anliegen der EZB ist. Letzterer würde dann trotz des Wunsches nach Reputation die Möglichkeit zu ihrer Realisierung fehlen, da die Politiker ihre stabilitätsbewußte Geldpolitik konterkarieren können. Insbesondere wenn die Zentralbank zur Produktion marktwidriger Erwartungseffekte in bezug auf den Wechselkurs gezwungen ist, wird sie aufgrund des Glaubwürdigkeitsdefizits dieser Politik an Reputation verlieren. Eine besondere Problematik entsteht zusätzlich, wenn nicht alle EU-Mitglieder auch Partner der Währungsunion sind, da dann Politiker Einfluß auf die Geldmengenentwicklung nehmen

22 Diese Forderung ist auch im Delors Bericht verankert. Vgl. Delors (1989), Punkt 32.

23 Vor der letzten Änderung des BBG sah dieses zwar noch die Möglichkeit von Kassenkrediten zur Überbrückung kurzfristiger Liquiditätsprobleme vor, da die Bundesbank die Kredite aber geben durfte und nicht mußte, handelte es sich bestenfalls um eine bedingte Einschränkung der Unabhängigkeit der Bundesbank. Vgl. auch Studt (1993), S. 80. 
können, deren Länder von diesen Beschlüssen gar nicht betroffen sind. Besser, weil reputationssteigernd, wäre hier eine Regelung, die die Zustimmung der EZB zur Wechselkurspolitik der Politiker verlangt. ${ }^{24}$

Neben der funktionellen ist die personelle Unabhängigkeit der EZB zu prüfen. Es wurden hohe fachliche Ansprüche an die Entscheidungsträger der Zentralbank gefordert, die explizit im Gesetz festzulegen sind, damit eine parteipolitische Durchdringung des Zentralbankrats verhindert wird. Zu beantworten sind auch die Fragen der Wahl, Dauer der Amtszeit sowie der Abberufungsmöglichkeiten. Schließlich sollte eine angemessene Dotierung einen Anreiz dafür bieten, daß tatsächlich die besten Fachkräfte zur Verfügung stehen.

Weder im BBG noch in der Satzung der EZB sind die Ansprüche an die Entscheidungsträger befriedigend geregelt. Das BBG fordert ganz allgemein eine besondere fachliche Eignung für die Mitglieder des Direktoriums und der Vorstände der Landeszentralbanken (\$7 II und 8 III). Diese „Floskel“" läßt erhebliche Interpretationsspielräume und kann kaum eine rein nach politischen Gesichtspunkten vorgenommene Besetzung verhindern, was die Besetzungspraxis besonders der Landeszentralbankpräsidenten, insbesondere in den letzten Jahren, immer wieder zeigt. Die Satzung der EZB (Artikel 11 II) wird hier zwar etwas präziser, indem sie, wie im Maastricht-Vertrag (Artikel 109 II b) selbst, das Direktorium nur mit Persönlichkeiten besetzen will, welche in Währungsoder Bankfragen anerkannt und erfahren sind. Eine solche Forderung kann aber nicht gewährleisten, daß hier tatsächlich ausschließlich geldpolitische Fachleute angesprochen sind.

Erschwerend kommt hinzu, daß diese „Ansprüche“ lediglich an die Mitglieder des Direktoriums gestellt werden, obwohl diese im Zentralbankrat in der Minderheit sein können. Entsprechend dem Maastricht-Vertrag (Artikel 109a II a bzw. Artikel 11 I der Satzung) besteht das Direktorium aus maximal sechs Mitgliedern, während jede nationale Zentralbank ihren Präsidenten in den europäischen Zentralbankrat schickt, der gleiches Stimmrecht hat wie ein Direktoriumsmitglied. Spätestens wenn die Währungsunion aus sieben Mitgliedern besteht, haben die Zentralbankpräsidenten die Stimmenmehrheit. Zu deren fachlicher Qualifikation sehen aber weder der Maastricht-Vertrag noch die Satzung der EZB irgendwelche Forderungen vor. Es wird lediglich die allgemeine Aussage getroffen, daß bis zur Errichtung der EZB die innerstaatlichen Vorschriften bzw. Satzungen der nationalen Zentralbanken der Mitgliedsstaa(1993), S. 97, Herr/Westphal (1991), S. 87 und Studt (1993), S. 262. 
ten im Einklang mit dem Maastricht-Vertrag bzw. der Satzung der EZB stehen sollen (Artikel 108 bzw. 14 I). Artikel 109e V des Vertrags wird etwas präziser, indem er die Einleitung der notwendigen Maßnahmen zur Unabhängigkeit der nationalen Zentralbanken in der zweiten Stufe vorsieht. Ihre volle Unabhängigkeit müssen die nationalen Zentralbanken jedoch erst zu Beginn der dritten Stufe, wenn die EZB ihre Arbeit aufnimmt, erhalten. Wie diese Unabhängigkeit aber z.B. in personeller Hinsicht aussehen soll, darüber werden kaum weitere Angaben gemacht, und letztlich bleibt die Auswahl der nationalen Zentralbankpräsidenten allein die Angelegenheit der Länder.

Aber auch die Auswahl und Ernennung der Direktoriumsmitglieder ist ausschließlich eine Aufgabe von Politikern. Artikel 109 II b des Maastricht-Vertrags bzw. Artikel 11 II der Satzung ordnen die Bestimmung des Direktoriums den Staats- und Regierungschefs zu. Der EZB-Rat kann hierzu, wie der deutsche Zentralbankrat, lediglich gehört werden. ${ }^{25}$ Allerdings ist eine relativ lange Amtszeit von acht Jahren vorgesehen, und eine Wiederernennung ist im Gegensatz zum BBG verboten (Artikel 11 II). Diese beiden Bestimmungen tragen zur persönlichen Unabhängigkeit positiv bei. Eine Beeinträchtigung dieses positiven Effektes liegt allerdings in der Bestimmung über die Amtsdauer der nationalen Zentralbankpräsidenten. Diese muß nur mindestens fünf Jahre betragen und kann deshalb in einigen Mitgliedsstaaten der Legislaturperiode einer gewählten Regierung entsprechen. Liegt eine solche Kombination vor, kann die Besetzungsfrage wieder primär politisch motiviert sein.

Unbefriedigend ist auch die Frage der Abwahl sowohl der Direktoriumsmitglieder als auch der nationalen Zentralbankpräsidenten gelöst. Beide können entlassen werden, wenn sie die Voraussetzungen für die Ausübung ihres Amtes nicht mehr erfüllen oder schwere Verfehlungen begangen haben (Artikel $11 \mathrm{IV}$ und 14 II). Eine solche Formulierung läßt erhebliche Interpretationsspielräume und räumt den Politikern die Möglichkeit ein, sich ,unbequemer" Zentralbanker vorzeitig zu entledigen. Zwar wird den nationalen Zentralbankpräsidenten die Möglichkeit zur Klage gegen ihre Entlassung vor dem Europäischen Gerichtshof zugestanden, die reale Wirksamkeit ein solchen Schutzes ist aber fraglich. Die Möglichkeit, die Präsidenten in Frankreich oder Italien jederzeit abzusetzen, wird durch eine solche Vorschrift de facto kaum beeinträchtigt.

25 Dieses Anhörungsrecht hat faktisch aber keinerlei Bedeutung, wenn die Politiker anderer Meinung sind. So hat sich der deutsche Zentralbankrat z.B. nicht nur das erste Mal gegen Hans-Jürgen Koebnick als Präsidenten der saarländischen Landeszentralbank ausgesprochen, sondern auch bei dessen Wiederbestellung. Trotzdem wurde dieser von den Politikern wieder ernannt. Diese Berufungspraxis könnte als Indiz für den Versuch der Politisierung des Zentralbankrats gewertet werden. 
Schließlich bleibt noch die Frage der angemessenen Dotierung. Diese läßt die Satzung der EZB aber bewußt offen. Für die Gehälter der nationalen Präsidenten ist keine Angabe gemacht, und die Gehälter sowie weitere Beschäftigungsbedingungen der Direktoriumsmitglieder sind gemeinsam von den Zentralbankpräsidenten festzulegen (Artikel 11 III).

Als dritte Ebene der rechtlichen Unabhängigkeit ist noch die finanzielle Seite zu analysieren, und hier insbesondere die Frage der Gewinnverteilung. Die Gewinnverteilung für die Bundesbank und die EZB entsprechen sich weitestgehend. Das BBG bestimmt in $\$ 27$ die Zuführung des Gewinnes in Rücklagen, die noch näher spezifiziert werden. Nur wenn dann noch ein Rest verbleibt, ist dieser dem Bund als Eigentümer zu übertragen. Ähnlich bestimmt Artikel 33 der Satzung die Gewinnverwendung für einen allgemeinen Reservefonds und die Abgabe des Restbetrags an die Eigner, die nationalen Regierungen. Eine Einflußmöglichkeit der Begünstigten auf die Gewinnhöhe besteht zumindest direkt nicht, da die Zentralbank ihre Geldpolitik unabhängig von den nationalen Regierungen bestimmt. Indirekt könnte solch ein Einflußnahmeversuch jedoch über die personelle Schiene erfolgen, indem ein Zentralbanker eingesetzt wird, der als nicht so inflationsavers gilt und die ,zugelassene“ Inflation dann zu zusätzlichen Einnahmen für die Regierung führt. Allerdings sind hier wieder die dafür notwendigen Mehrheitsverhältnisse zu berücksichtigen. ${ }^{26}$

Zusammenfassend bleibt festzuhalten, daß die rechtlich verankerte Unabhängigkeit der EZB genau wie die der Bundesbank nicht ausreichend ist, um das Ziel der Preisniveaustabilisierung zu gewährleisten. Zudem bleiben viele Interpretationsspielräume, so daß die tatsächliche Unabhängigkeit je nach informeller Macht, die einer Zentralbank von der Bevölkerung verliehen wird, erheblich von der juristischen Unabhängigkeit abweichen kann.

Die Bundesbank konnte trotz unzureichender gesetzlicher Grundlage eine äußerst hohe tatsächliche Unabhängigkeit erzielen, da die deutsche Bevölkerung das Ziel der Bundesbank untrennbar mit deren Unabhängigkeit verbindet. Dies ist aber nur eine denkbare Alternative für die tatsächliche Unabhängigkeit, die durch die Interessengleichheit von Bundesbank und Wirtschaftssubjekten begründet wird. Nur weil die stabilitätsbewußte Bundesbank ihre Geldpolitik für eine inflationsaverse Bevölkerung durchführt, ist ihre Unabhängigkeit nicht gefährdet, selbst wenn die Regierung von Politikern gestellt wird, die andere Ziele bevorzugen.

26 Vgl. Gäckle (1992), S. 98, Leigh-Pemberton (1990), S. 46, Caesar (1982), Hasse (1989a), S. 209ff. und Berthold (1992), S. 27f. 
Wie „unangreifbar“ und somit unabhängig die Bundesbank letztlich ist, zeigte sich besonders in den siebziger Jahren, als immer wieder Vorschläge von politischer Seite kamen, die Macht der Bundesbank einzuschränken und das BBG entsprechend zu ändern. Wie dagegen die tatsächliche Unabhängigkeit der EZB aussehen wird, ist ungewiß. Im theoretischen Teil wurde gezeigt, daß die De-facto-Unabhängigkeit wesentlich von den politischen Zwängen, die auf die Zentralbank ausgeübt werden, abhängt. Die politischen Zwänge bzw. Beeinflussungsversuche hängen wiederum vom Konfliktpotential und der Konfliktbereitschaft der beteiligten Akteure ab.

Das Konfliktpotential in Europa wird aber höher sein als das in Deutschland, allein schon aufgrund der Größenverhältnisse. Auch wird auf europäischer Ebene die Interessenvielfalt zunehmen. Die Geldpolitik muß dann nicht nur die wirtschaftliche Lage eines Landes berücksichtigen, sondern die vieler Länder.

Zudem ist keine europäische Fiskal- oder Tarifpolitik vorgesehen. Beide bleiben weiterhin in nationaler Verantwortung, und eine „Abstimmung“ mit der Geldpolitik wird schwieriger. Zwar wurde im Maastricht-Vertrag festgelegt, daß die Gemeinschaft nicht für Verbindlichkeiten der Zentralregierungen haftet (Artikel 104b I), es ist jedoch kaum realistisch, daß ein überschuldetes Mitglied nicht die Solidarität der Partner erfährt. Einen ,geräuschlosen“ Weg, die Fehler einer zu expansiven Fiskalpolitik zu verdecken, bietet die Geldpolitik, durch eine Inflationierung. Der Druck auf die Zentralbank kann also erheblich sein. Ein institutionelles Einfallstor für zunehmende politische Pressionen bietet der Artikel 2 der EZB-Satzung, der die Unterstützung der allgemeinen Wirtschaftspolitik fordert. Eine akkommodierende Geldpolitik würde aber das Vertrauen in die Geldpolitik bzw. die EZB erschüttern, und letztlich könnten dann doch nur unerwünschte Zinssteigerungen zur Wiedererlangung der Reputation führen.

Eine Möglichkeit, das Konfliktpotential zu mindern, besteht in der Vorgabe und Veröffentlichung von monetären Zwischenzielen, welche in der EZB-Satzung zumindest vorgesehen sind (Artikel 12 I). An diesen können sich dann nicht nur alle wirtschaftspolitischen Partner orientieren, auch den Wirtschaftssubjekten wird ein klares Signal gegeben, was die Zentralbank zu realisieren beabsichtigt. Reputationssteigernd wirkt diese Strategie allerdings nur langfristig, wenn die Zentralbank bewiesen hat, daß sie ihre Ankündigungen auch einhält. Weicht sie hingegen ständig von ihrem selbstgesteckten Ziel ab, kann dies eher reputationsmindernd wirken. 
Aber nicht nur das steigende Konfliktpotential der Geldpolitik stellt für die tatsächliche Unabhängigkeit der EZB eine Gefahr dar, auch die steigende Konfliktbereitschaft ihrer "Gegner" wirkt in die gleiche Richtung. Die EZB wird sich nicht wie die Bundesbank nur einer nationalen Regierung, welche die Interessen eines Volkes zu berücksichtigen hat, und einer nationalen Tarifpolitik gegenübersehen. Mehrere Regierungen, die unterschiedliche Bevölkerungen mit verschiedenen Inflationserfahrungen vertreten, und viele Gewerkschaften mit unterschiedlicher Einsichts- bzw. Streikbereitschaft müssen die stabilitätskonforme Geldpolitik der EZB unterstützen. ${ }^{27}$

Die Konfliktbereitschaft der Zentralbank ist hingegen ungewiß. Die Bundesbankspitze weiß die Bevölkerung hinter sich und kann Konflikte mit der Regierung riskieren. Die Mentalität bzw. Inflationsaversion der europäischen Bevölkerung ist aber nicht einheitlich. Auch ist wichtig, ob Politiker oder Fachleute in die Zentralbank berufen werden. Wie gezeigt wurde, ist es nicht ausgeschlossen, daß auch Politiker die europäische Geldpolitik bestimmen werden, und die weisen eine geringere Konfliktbereitschaft gegenüber den Regierungen auf. Die Auswahl insbesondere der ersten Zentralbankratsmitglieder wird ein wichtiges Signal für die Wirtschaftssubjekte sein, wie ernst es den Politikern damit ist, daß die EZB primär die Geldwertstabilität sichern bzw. ihre Reputation maximieren soll.

Die tatsächliche Unabhängigkeit der EZB als reputationssteigernde Maßnahme ist bisher also ungewiß. Die gesetzlichen Regelungen reichen hierfür nicht aus, und wie es mit der Umsetzung der juristischen Unabhängigkeit in die Realität aussehen wird, ist fraglich, da ein steigendes Konfliktpotential in Verbindung mit der steigenden Konfliktbereitschaft der anderen Wirtschaftspolitiker zu erwarten ist, die Konfliktbereitschaft der Zentralbanker selbst aber erst nach deren Wahl durch die Politiker beurteilt werden kann.

Nach den bisherigen Ausführungen ist nicht zu erwarten, daß die EZB mit einer ähnlich hohen Reputation starten wird, wie die Bundesbank sie bereits besitzt. Zudem wurde bisher implizit davon ausgegangen, daß alle Zentralbanker ihre Reputation automatisch steigern wollen, wenn sie nur tatsächlich unabhängig agieren können. Wie im theoretischen Teil gezeigt wurde, ist dies aber selbst für Fachleute nicht zu unterstellen. Entsprechend der Neuen Institu-

27 Eine hierzu kontroverse Beurteilung findet sich bei Bofinger (1993), S. 45, der die Meinung vertritt, daß die EZB als supranationale Zentralbank einem geringeren politischen Druck ausgesetzt sein wird als eine nationale Zentralbank, da die Forderungen eines einzelnen Landes weniger wiegen. Die Schaffung der EZB bedeute gleichsam eine „Entnationalisierung des Geldes“. 
tionenökonomie will jedes Wirtschaftssubjekt seinen Eigennutz maximieren, und dies gilt auch für die Zentralbanker.

Wie ausgeführt wurde, sind letztlich alternativ drei primäre Ziele für Zentralbanker denkbar. Die Reputation ist hiervon nur eines. Ein Zentralbanker könnte auch ein Interesse an der Erzielung von Prestige oder Macht haben. Es wurde gezeigt, daß nur unter ganz bestimmten Umständen auch die anderen Ziele teilweise den Wunsch nach Reputation beinhalten.

Nur bei einer inflationsaversen Bevölkerung wird der Wunsch eines unabhängigen Zentralbankers nach Prestige über den Aufbau von Reputation erfüllt. Liegt die Machterzielung im Eigeninteresse des Zentralbankers, muß er schon selbst stabilitätsbewußt sein. Sieht das Zentralbankstatut dann auch noch eine Erweiterung des Handlungsspielraums der Zentralbanker vor, indem der Aufgabenkatalog um reale Ziele erweitert ist, impliziert eine Verletzung des Stabilitätsziels noch nicht einmal eine Pflichtverletzung, da die Verfehlung der Preisniveaustabilität dann mit Maßnahmen begründet werden kann, die andere Ziele begünstigen. Letztlich ermöglicht eine Vorgabe mehrerer Ziele, die womöglich kurzfristig miteinander konkurrieren, dem Zentralbanker die Rechtfertigung jeder Handlung, und der Wunsch bzw. der Zwang zum Aufbau von Reputation wird um so unwahrscheinlicher. ${ }^{28}$

Da kein Zentralbanker seine persönlichen Präferenzen offenlegen wird und der Wunsch, sich Reputation aufzubauen, immer nur einen Spezialfall darstellt, müßte die Satzung der EZB externe Anreize für die Zentralbanker beinhalten, die sie persönlich treffen, damit diese auch mit Sicherheit ihre Reputation steigern wollen. Die Formen der theoretischen Anreize wurden bereits im theoretischen Teil beschrieben.

\subsection{Anreize für die EZB}

Wie gezeigt wurde, existieren für eine Zentralbank keine wirksamen marktähnlichen oder politischen Kontrollmechanismen. Letztlich können nur persönlich wirkende Anreize die Zentralbanker zu einem stabilitätskonformen Verhalten ,zwingen“, da auch die schwächeren Formen der Anreize, wie der Abbau der Informationsasymmetrie und die Einführung einer Regelbindung, ein solches Verhalten nicht gewährleisten können. ${ }^{29}$

Vgl. Hasse (1989a), S. 137, Tietmeyer (1992), S. 20, Gäckle (1992), S. 96, Scharrer (1988), S. 99, ders. (1989), S. 282, Herr/Westphal (1991), S. 90f. und Issing (1992c), S. 7.

Vgl. hierzu Kapitel 3.4. 
Aber weder der Maastricht-Vertrag noch die Satzung der EZB sehen irgendwelche persönlichen Anreize für die Zentralbanker vor, damit es in deren primärem Interesse liegt, ihre Reputation zu steigern. Selbst dauerhafte Zielverfehlungen führen für die Verantwortlichen der Geldpolitik zu keinerlei Sanktionen. Die Gesetzgeber sind offensichtlich davon ausgegangen, daß solche Regelungen nicht notwendig sind, weil für sie allein die Unabhängigkeit eine notwendige und bereits hinreichende Bedingung für den Reputationsaufbau ist. Dies belege das deutsche Beispiel, da im BBG auch keine externen Anreize vorgesehen sind und die Bundesbank trotzdem eine solch hohe Reputation erzielt hat. Hierbei übersehen die Politiker jedoch, daß nicht allein die Unabhängigkeit zu dieser hohen Reputation geführt hat, sondern die anderen günstigen Rahmenumstände. Insbesondere die ausgeprägte Stabilitätskultur der deutschen Bevölkerung, die die Erfahrung zweier großer Inflationen hat, ist ein wesentlicher Faktor des Erfolges der Bundesbank. Ob alle anderen Partner, die an der Währungsunion teilnehmen werden, ebensolche günstigen Ausgangsbedingungen mitbringen, ist zumindest zweifelhaft. ${ }^{30}$

Diese Befürchtungen müssen auch die Gesetzgeber gesehen haben, da sie ihnen durch die Aufstellung von Konvergenzkriterien meinten begegnen zu können, welche ein Land für die Teilnahme an der Währungsunion erfüllen muß. Die Formulierung dieser Kriterien kann als reputationsfördernde Maßnahme für die EZB gesehen werden, da so gezeigt werden soll, daß nur Länder die Währungsunion bilden, denen es tatsächlich ernst ist mit der Geldwertstabilität. Ob die aufgestellten Kriterien diesem Anspruch gerecht werden, soll nun näher geprüft werden.

Die Konvergenzkriterien sind im Artikel $109 \mathrm{j}$ I des Maastricht-Vertrags festgelegt und werden in Zusatzprotokollen über die Konvergenzkriterien bzw. über das Verfahren bei einem übermäßigen Defizit näher spezifiziert. Danach müssen die potentiellen Mitglieder einen hohen Grad an Preisstabilität erzielt haben, der aus einer Inflationsrate ersichtlich wird, die der Rate der drei Länder nahe kommt, die auf diesem Gebiet das beste Ergebnis erzielt haben. Im Protokoll wird zwar der Ausdruck ,nahe kommen" konkretisiert, indem eine Abweichung von 1,5 Prozentpunkten von der Vergleichsrate gewählt wird, die Höhe dieser Vergleichsrate bleibt aber ungeregelt. Man findet keine Aussage darüber, ob hier als Maßstab das schlechteste der drei besten Länder oder ein Durchschnitt genommen wird. Zudem muß diese Bedingung auch nur ein Jahr vor der Prüfung der Kriterien erfüllt sein. Hier besteht der Anreiz, zum Prüftermin seine Inflationsrate kurzfristig zu senken, damit das Kriterium erfüllt ist.

Vgl. hierzu auch Abschnitt 4.1 und 4.2. 
Welche Höhe die Inflationsraten aber vor und nach dem Prüftermin haben, scheint den Gesetzgebern unwichtig gewesen zu sein.

Ebenso unzureichend wie dieses sind auch die drei nachfolgenden Kriterien geregelt. So wird eine „tragbare Finanzlage der öffentlichen Hand“ gefordert, die durch das Fehlen eines übermäßigen Defizits spezifiziert ist. Wann ein übermäßiges Defizit vorliegt, wird durch Artikel 104c II des Vertrags bzw. durch das Protokoll über das Verfahren bei einem übermäßigen Defizit festgelegt. Danach darf weder das Verhältnis des geplanten oder tatsächlichen Defizits noch das des öffentlichen Schuldenstandes zum Bruttoinlandsprodukt einen bestimmten Referenzwert überschreiten. Die Referenzwerte wurden (willkürlich) mit 3\% bzw. $60 \%$ festgelegt. Es gelten aber Ausnahmen von diesen Regeln, wenn die Verhältnisse rückläufig sind und sich ,in der Nähe“ der Referenzwerte befinden. Des weiteren wird auch geprüft, ob der Referenzwert eventuell nur ausnahmsweise überschritten wird.

Das dritte Kriterium fordert die „Einhaltung der normalen ${ }^{31}$ Bandbreiten des Wechselkursmechanismus“ des EWS seit mindestens zwei Jahren. Die Währung darf in dieser Zeit gegenüber einem anderen Mitglied nicht abgewertet worden sein. Insbesondere dieses Kriterium zeigt die Sinnlosigkeit dieser Forderungen. Bei strenger Auslegung dürfte es gar nicht mehr zur Währungsunion kommen, da die „normalen“ Bandbreiten von 2,25\% im Zuge der Währungskrise 1992 aufgegeben wurden und durch eine Abweichungsmöglichkeit von $15 \%$ nach oben und unten ersetzt wurden. ${ }^{32}$ De facto liegt hier zumindest aus ökonomischer Sicht kein Festkurssystem mehr vor. Bei politischer Interpretation der „normalen“ Bandbreite von tatsächlich 30\% wird jedes Mitgliedsland in der Lage sein, dieses Kriterium zu erfüllen. Die positive Wirkung auf die Reputation ist aber komplett aufgehoben. Sie kann sich dadurch sogar in die negative Richtung umkehren.

Schließlich müssen sich auch die langfristigen Zinssätze der Mitglieder angenähert haben. Wie schon beim ersten Kriterium wird auch hier ein Maßstab gewählt, den die drei „besten“ Länder vorgeben. Die Abweichung vom Referenzwert darf $2 \%$ nicht überschreiten. Wieder bleibt ungeklärt, ob es sich um einen Durchschnittswert handeln soll oder der höchste der drei geringsten Zinssätze als Maßstab dient. Auch dieses Kriterium muß wieder nur in einem Jahr erfüllt sein. Die These, daß aufgrund der Konvergenzkriterien also nur die Länder an der Währungsunion teilnehmen, die über mehrere Jahre bereits eine

31 Hervorhebung durch den Autor.

32 Der Franz-Bericht des Europaparlaments sah vor, die zulässige Schwankungsbreite im EWS bis 1995 stufenweise auf Null zu verringern. 
stabilitätsorientierte Wirtschaftspolitik verfolgen, welche zudem von der Bevölkerung gestützt und deshalb als Indiz für die Entwicklung einer Stabilitätskultur entsprechend der deutschen gewertet werden $\operatorname{kann}^{33}$, muß hier als nicht gerechtfertigt abgelehnt werden. Der im Maastricht-Vertrag gewählte Zeitraum von einem Jahr reicht kaum als Grundlage für eine Beurteilung aus, ob eine Stabilitätsgemeinschaft erreicht wurde.

Vollends werden diese ohnehin sehr weit interpretierbaren Kriterien, nach ihrer ausführlichen Darstellung, schließlich wieder aufgehoben, indem anschließend im zweiten Absatz des Artikels 109j bestimmt wird, daß der Rat erst mit qualifizierter Mehrheit bestimmen muß, ob die Kriterien erfüllt sind oder nicht. Man muß sich hier fragen, warum zunächst Werte festgelegt werden, wenn diese dann doch nicht gelten sollen, da letztlich politisch entschieden wird. Sollte die politische Entscheidung die aufgestellten Werte tatsächlich nicht berücksichtigen, wirkt dies negativ auf die Reputation der EZB.

Aber auch die präzise Terminvorgabe, daß die EZB spätestens am 1. Januar 1999 gegründet werden müsse, paßt nicht zu den aufgestellten Kriterien. Es ist auch keine Regelung für den Fall vorgesehen, daß keines der Länder bis zu diesem Termin die Kriterien erfüllt. Berücksichtigt man nun auch noch den Wunsch der Politiker, mit einer möglichst großen Teilnehmerzahl zu starten, relativiert dies die Chancen für die Einhaltung der Kriterien noch weiter.

Wenn die EZB mit einer möglichst hohen Reputation starten soll, müßten die Politiker auf der strikten Einhaltung der Kriterien bestehen, selbst wenn diese ökonomisch nicht begründbar sind. Sie wurden aber zur „Meßlatte“ einer Teilnahme erklärt, und dann sollten sie auch eingehalten werden, da sonst das Vertrauen in die neue Institution leiden würde. Wenn hierfür der bestimmte Termin verschoben werden müßte, würde diese Flexibilität der Politiker nur den Wunsch nach Reputation für die EZB belegen. ${ }^{34}$

33 Vgl. hierzu Bofinger (1993).

34 Vgl. Vaubel (1989), S. 278, ders. (1993), S.59, Gischer (1993), S. 50, Caesar (1990), S. 116, Tietmeyer (1993), S. 34, Berthold (1992), S. 27f., Hasse (1992), S. 29, ders. (1993a), S. 132 ff., ders. (1993b), S. 2. 
Iris Henning - 978-3-631-75125-1

Downloaded from PubFactory at 01/11/2019 08:02:27AM

via free access 


\section{Zusammenfassung und abschließende Beurteilung}

Das Ziel dieser Arbeit war es, zunächst die Bedeutung der Reputation für eine Zentralbank zu zeigen und im zweiten Schritt herauszuarbeiten, wie die Reputation aufgebaut werden kann, um Anhaltspunkte für die Reputation der zukünftigen EZB zu erhalten.

Das grundlegende Modell zur Reputation haben Barro/Gordon beschrieben, weshalb dieses zu Beginn des analytischen Teils in Kapitel 2 vorgestellt wurde. Hiermit konnte gezeigt werden, daß trotz eines diskretionären Handlungsspielraums der Zentralbank nicht automatisch die „schlechte“ diskretionäre Inflationsrate realisiert wird. Dies war das Ergebnis der bahnbrechenden Arbeit von Kydland/Prescott, die nachgewiesen hatten, daß bei diskretionärem Handlungsspielraum nie die optimale Inflationsrate erreicht werden kann. Der Grund dafür lag im Anreizproblem der Geldpolitik, von der ex ante optimal erscheinenden Inflationsrate abzuweichen, sobald die Wirtschaftssubjekte an deren Realisation glauben und sich durch ihre Lohnverträge an diese gebunden hätten. Würden die Geldpolitiker dann doch eine höhere Inflationsrate setzen, könnte die resultierende Überraschungsinflation das Problem der aufgrund marktverzerrender Einflüsse als zu hoch bewerteten Arbeitslosigkeit zumindest kurzfristig mindern.

Da auch die Wirtschaftssubjekte dieses Anreizproblem der Politiker kennen, werden sie immer die „schlechteste“ Inflationsrate antizipieren, um nicht von den Politikern „,betrogen“ zu werden. Die Wahl dieser „schlechten“ Inflationsrate führt dann aber aufgrund des fehlenden Überraschungseffektes nicht einmal zur Steigerung der Beschäftigung. Die einzig konsistente Lösung dieses Modells führt für die Gesellschaft zu Wohlfahrtseinbußen, da das resultierende Nash-Gleichgewicht kein Pareto-Optimum darstellt.

Zur Lösung dieser Gefangenendilemma-Situation wurde zunächst gefordert, aus dem nicht-kooperativen Spiel (diskretionäre Politik) ein kooperatives zu machen, indem die Geldpolitik an fest vorgegebene Regeln gebunden wird. Durch diese Beseitigung des diskretionären Handlungsspielraums der Geldpolitiker könnten die Wirtschaftssubjekte sich darauf verlassen, da $\beta$ die angekündigte geringe Inflationsrate auch realisiert wird, und als Lösung ergibt sich tatsächlich eine geringere Inflationsrate bei gleichbleibender Arbeitslosenrate.

Betrachtet man jedoch die Geldpolitik als fortlaufenden Prozeß und berücksichtigt, daß die geldpolitischen Entscheidungen und die Erwartungsbildung der Wirtschaftssubjekte in einem kontinuierlichen Wechselspiel zueinander stehen, wird ein intertemporaler Ansatz erforderlich, der die Ergebnisse relati- 
vieren kann. Wird berücksichtigt, daß der heutige Betrug der Politiker nicht nur einen Nutzen in Form temporär sinkender Arbeitslosigkeit stiftet, sondern auch Kosten verursacht, die in Form steigender Inflationserwartungen in der Zukunft zum Ausdruck kommen, nimmt der Anreiz zur Inflation ab. Der drohende Anstieg der Inflationserwartungen wird als Reputationsverlust interpretiert. Diese Sanktion ermöglicht die Realisation einer geringeren Inflationsrate als der diskretionären, auch wenn der diskretionäre Handlungsspielraum der Politiker bestehen bleibt. Die Höhe der realisierbaren Inflationsrate ist dabei abhängig von den Zielpräferenzen der Politiker sowie deren Zeitpräferenzrate, die auch die Wirtschaftssubjekte kennen. Es ist also die Reputation, die letztlich über den Erfolg einer Antiinflationspolitik entscheidet.

In diesem Grundmodell beschreibt die Erfüllung der Erwartungen eine glaubwürdige Geldpolitik, die dann zum Reputationsaufbau führt. Letzterer spiegelt sich in geringen Erwartungen für die nächste Periode wider. Da in diesem Modell aber ausschließlich die Erfüllung der Erwartungen unabhängig von deren Höhe zum Reputationsaufbau führt, wurde anschließend eine Erweiterung des BG-Modells vorgestellt, in der nur eine Antiinflationspolitik zum Aufbau der Reputation beiträgt.

Es wurde nun erforderlich, die beiden häufig synonym verwendeten Begriffe Reputation und Prestige voneinander abzugrenzen. Die Reputation wurde definiert als Ruf der Zentralbank in der Öffentlichkeit, ein Inflationsgegner zu sein. Unabhängig davon, was die Bevölkerung sich von der Zentralbank wünscht, muß sie diese „nur“ als stabilitätsorientiert einstufen, damit sie Reputation besitzt. Sehen die Wirtschaftssubjekte hingegen ihre eigenen Präferenzen von der Zentralbankpolitik erfüllt, werden sie diese mit Prestige belohnen, unabhängig davon, ob das Ziel Geldwertstabilität oder Bekämpfung der Arbeitslosigkeit von der Bevölkerung bevorzugt wird.

Diese Unterscheidung hat zur Folge, daß eine Zentralbank nur unter bestimmten Umständen durch die Erzielung von Prestige auch Reputation aufbauen kann. Nur wenn die Bevölkerung eine inflationsaverse Zentralbank wünscht, sind Reputation und Prestige über die gleiche Politik zu erzielen. Da diese Differenzierung eine wesentliche Grundlage dieser Arbeit darstellt, wurde hierauf im Abschnitt 2.2 ausführlich anhand mehrerer Fallunterscheidungen eingegangen. Des weiteren wurde noch der Begriff Glaubwürdigkeit erläutert, der sich auf die Politik selbst bezieht und im Rahmen dieser Arbeit immer als Antiinflationspolitik verstanden wurde. 
Nach Klärung dieser Begriffe wurde dann das modifizierte Modell vorgestellt, welches durch die Annahme eigennutzorientierter Zentralbanker den Erkenntnissen der Neuen Politischen Ökonomie Rechnung trägt. Eine Folge dieser neuen Betrachtungsweise war die Aufgabe der Unterstellung symmetrischer Informationen, da die Zentralbank nun aufgrund sich wandelnder Präferenzen im Zeitablauf einen Informationsvorsprung hat, der nie ganz abgebaut werden kann.

Zwar kündigt die Zentralbank auch hier ihre nach der BG-Formel errechnete Inflationsrate an, da die Wirtschaftssubjekte aber aufgrund unvollkommener Informationen eine andere Meinung über das Stabilitätsbewußtsein bzw. der Zeitpräferenzrate der Zentralbank haben können, müssen die Wirtschaftssubjekte diese Rate nicht als glaubwürdig erachten. Zudem wissen sowohl die Zentralbanker als auch die Wirtschaftssubjekte, daß erstere die Inflationsrate nicht allein setzen können, sondern nur den Teil bestimmen, der durch die Geldpolitik verursacht wird. Die tatsächlich realisierte Inflationsrate wird zumindest kurzfristig insbesondere auch von der Fiskalpolitik der Regierung bestimmt. Da dies aber auch die Wirtschaftssubjekte wissen, berücksichtigen sie diese Tatsache bei der Festlegung der Reputation für die Zentralbank, die nur für die eigene Leistung verantwortlich gemacht wird. Ein weiteres Unterscheidungsmerkmal zum Basismodell kommt durch die Definition der Glaubwürdigkeit als Übereinstimmung der Ankündigung mit den Erwartungen zum Ausdruck.

Glauben die Wirtschaftssubjekte die Ankündigung der Zentralbank, wird sie diese auch realisieren, wenn sie sich Reputation aufbauen will. In diesem Fall führt also auch hier die Erfüllung der Erwartungen zum Reputationsaufbau. Es ist aber auch denkbar, daß die Wirtschaftssubjekte der angekündigten Rate keinen Glauben schenken und eine höhere Inflationserwartung haben. In diesem Fall der unglaubwürdigen Geldpolitik ist es für die Zentralbank nicht mehr optimal, sich an die eigene Ankündigung zu halten. Zur Berechnung der ex post optimalen Inflationsrate müssen neben den eigenen Zielpräferenzen und dem Diskontierungsfaktor nun auch die Inflationserwartungen berücksichtigt werden, da jede von diesen abweichende Inflationsrate nun zu Kosten in Form einer Stabilisierungskrise führt, die zu berücksichtigen sind. Will sich die Zentralbank in diesem Fall Reputation aufbauen, muß sie die Erwartungen der Wirtschaftssubjekte enttäuschen und eine geringere Rate wählen, die aber aufgrund der „hohen“ Erwartungen oberhalb der Inflationsrate bei glaubwürdiger Politik liegt. Die Folge sind ein kurzfristiges Steigen der Arbeitslosigkeit und der Verzicht auf Prestige, falls die Erwartungen der Wirtschaftssubjekte deren Wünschen entsprechen. 
Der Aufbau von Reputation bei unglaubwürdiger Geldpolitik stellt die Zentralbank demnach vor ein größeres Problem, als wenn ihre Geldpolitik für die Wirtschaftssubjekte glaubwürdig ist. Die Glaubwürdigkeit stellt also eine wesentliche Bedingung für die Reputation dar. Die beiden weiteren die Reputation bestimmenden Variablen sind die Stabilitätsorientierung sowie die Zeitpräferenzrate der Zentralbank.

Im modelltheoretischen Teil konnten die Bedeutung der Reputation nachgewiesen und deren direkte Determinanten herausgearbeitet werden. Im dritten Kapitel wurde dann beschrieben, wie diese die Reputation (direkt) bestimmenden Einflußgrößen beeinflußt werden können (indirekte Determinanten). Für die weitere Untersuchung wurde aufgrund der stärkeren Betonung der Neuen Politischen Ökonomie der verbal-analytischen Methode der Vorzug gegeben.

Es konnte gezeigt werden, daß eine wesentliche Bedingung für eine hohe Reputation die Unabhängigkeit der Zentralbank ist. Solange die geldpolitischen Entscheidungen der Zentralbank von einer wahlabhängigen Regierung dominiert sind, werden die Wirtschaftssubjekte eine konsequente Antiinflationspolitik kaum für realistisch halten. Der Grund hierfür ist in den Inflationsanreizen, insbesondere den kurzfristigen Beschäftigungseffekten sowie den Umverteilungseffekten, denen die Regierung aufgrund ihrer Wiederwahlrestriktion unterliegt, zu sehen. Nur die Loslösung der Geldpolitik aus dem tagespolitischen Geschehen, was eine unabhängige Zentralbank erfordert, kann eine Antiinflationspolitik auch in langer Sicht glaubwürdig erscheinen lassen und somit zum „kostenlosen“ Reputationsaufbau beitragen.

Die Unabhängigkeit der Zentralbank ist allerdings als Mittel zum Zweck zu sehen, insofern als sie die Sicherung des Geldwertes garantieren soll und deshalb funktionsbezogen festzuschreiben ist. Die Zentralbank muß also per Gesetz ausschließlich auf das Ziel der Inflationsbekämpfung verpflichtet werden. Diese Beschränkung muß aber im funktionellen Bereich der Unabhängigkeit die einzige bleiben. Vor allem muß auf dieser Ebene die instrumentelle Unabhängigkeit gewährleistet sein. Einen kritischen Punkt stellt hier die Entscheidungskompetenz über die Wechselkurspolitik dar, weil sie eine binnenwirtschaftlich antiinflationäre Geldpolitik konterkarieren könnte und deshalb in den Kompetenzbereich der Zentralbank gehört.

Neben der funktionellen sind aber auch die personelle und finanzielle Unabhängigkeit zu gewährleisten. Insbesondere über die personelle Ebene versuchen die Politiker bei de jure unabhängigen Zentralbanken Einfluß zu nehmen, wie die Erfahrung zeigt. Deshalb ist vor allem eine hohe fachliche Eignung der 
Zentralbanker zu fordern, die explizit im Gesetz so zu konkretisieren ist, daß mögliche Interpretationsspielräume ausgeschlossen sind. Um dennoch ein politisches „Wohlverhalten“ auszuschließen, sind lange Amtszeiten ohne die Möglichkeit der Wiederbestellung sowie der vorzeitigen willkürlichen Abberufung nötig. Insbesondere sollten nicht Politiker über die Bestellung der Zentralbanker bestimmen dürfen.

Aber selbst eine nahezu perfekte Regelung der Unabhängigkeit im Gesetz, die eventuell sogar de facto realisiert werden kann, führt nicht automatisch zum Reputationsaufbau der Zentralbank. Die Unabhängigkeit der Zentralbank ist zwar eine notwendige, aber noch keine hinreichende Bedingung für eine maximale Reputation. Entsprechend den Erkenntnissen der Neuen Institutionenökonomie maximiert jede Institution ihren eigenen Nutzen, d.h. letztlich wird jeder einzelne Zentralbanker bei seinem Handeln von seinen persönlichen Zielen geleitet. Der Aufbau von Reputation ist hierbei neben anderen nur ein mögliches Ziel. Sobald ein Zentralbanker ein anderes Ziel verfolgt, muß durch entsprechende Rahmenbedingungen dafür gesorgt werden, daß schließlich doch nur die Reputationssteigerung seinem persönlichen Interesse dient.

Als Problem erweist sich die Tatsache, daß die Zentralbank mit der Preisniveaustabilität ein öffentliches Gut anbietet. Es wird deshalb nicht zur Bildung von Interessengruppen für dieses Gut kommen und somit auch nicht zur Kontrolle der Zentralbank. Da auch keine anderen marktähnlichen oder politischen Kontrollmechanismen auf die Zentralbank wirken, müssen ihr explizite Anreize zur Erreichung ihres Ziels vorgegeben werden. Von den drei diskutierten Möglichkeiten der Setzung externer Anreize konnte letztlich nur eine Form, die der persönlich wirkenden Anreize, die Zielrealisation garantieren. Weder ein verstärkter Zwang zur Veröffentlichung von Informationen noch die Einführung einer Regelbindung allein könnten dies gewährleisten. Eine Regelbindung erfüllt diesen Zweck erst, wenn zusätzlich Anreize bestehen, diese auch einzuhalten, also wieder persönliche Anreize für die Zentralbanker vorliegen.

Es wurden verschiedene Möglichkeiten dieser Anreize diskutiert, die sich je nach verfolgtem persönlichen Ziel eines Zentralbankers unterschieden. Die schärfste Form der Sanktionierung stellt die Entlassung bei Mißerfolg dar, die zugleich aber den Vorteil besitzt, daß sie immer wirkt, unabhängig davon, welches spezielle Ziel ein Zentralbanker verfolgt. Die Strafe sollte jedoch das einzelne Individuum treffen und nicht als Kollektivstrafe realisiert werden, so daß Inflationsgegner, die sich im Zentralbankrat mit ihrer Antiinflationspolitik nicht durchsetzen konnten, dafür nicht bestraft werden. Zudem ist zu berücksichtigen, daß die Zentralbank die Inflationsrate kurzfristig nicht allein kontrollieren 
kann, was durch eine längerfristige Zielvorgabe bzw. Berücksichtigung des politisch bedingten Inflationsanteils realisiert werden kann. ${ }^{1}$ Der Vorteil der expliziten Vorgabe auf die Zentralbanker persönlich wirkender Anreize ist schließlich nicht nur darin zu sehen, daß diese selbst ein Interesse an ihrem Reputationsaufbau haben, sondern daß auch die Wirtschaftssubjekte an eine stetige Antiinflationspolitik glauben und dann ihre Erwartungen entsprechend mindern. Eine Stabilisierungspolitik wird somit glaubwürdig, und der sonst „kostspielige“ Aufbau von Reputation ist nicht erforderlich, da sie über eine glaubwürdige Geldpolitik gesteigert werden kann.

Im vierten Kapitel wurde dann untersucht, ob die Rahmenbedingungen für die zukünftige EZB die theoretischen Erfordernisse zur Reputationssteigerung erfüllen bzw. ob sie eine Chance hat, mit der gleichen hohen Reputation zu starten, wie die Bundesbank sie bereits besitzt. Um die zu erwartende Leistung der neu zu gründenden EZB zu bestimmen, wurden die bisherigen Leistungen der potentiellen Partner als erster Anhaltspunkt untersucht. Die Betrachtung der Inflationsentwicklung führte zu einer Spaltung der Länder in mehrere Gruppen.

Der Bundesbank kann eine mehr oder weniger strikte Antiinflationspolitik seit ihrem Bestehen bestätigt werden, was die Ursache ihrer heutigen hohen Reputation ist. Aber auch Österreich und die Niederlande verfolgen schon seit längerer Zeit eine Stabilisierungspolitik. Eine zweite Ländergruppe, deren Inflationsrate erst seit Mitte der achtziger Jahre als Indiz für eine am Geldwert orientierte Geldpolitik gewertet werden kann, bilden Frankreich, Belgien und Luxemburg. Aber auch Irland und Dänemark können dieser Gruppe zugerechnet werden sowie seit Beginn der neunziger Jahre auch Finnland. Die Entwicklung in den übrigen Ländern ist nicht durch eine längerfristige Antiinflationspolitik gekennzeichnet. Zwar ist eine solche ansatzweise in Portugal, Spanien und Italien zu erkennen, aber das Inflationsniveau liegt noch deutlich über dem Durchschnitt der anderen Partner. Sehr viel extremer gilt diese Aussage für Griechenland, welches noch heute eine Rate von knapp unter $10 \%$ realisiert. Die unstetige Entwicklung in Schweden und Großbritannien zeugt nicht von einer Stabilitätspräferenz.

Die zu erwartende Leistung der EZB wird also im wesentlichen davon abhängen, welche Länder an der Währungsunion von Beginn an teilnehmen. Hierfür wurden im Kapitel 4.2 mehrere Szenarien betrachtet. Aber selbst wenn es zunächst nur zu einer Kern-Währungsunion kommen sollte, die sich nur aus

$1 \quad$ Vgl. hierzu die entsprechende Regelung im Statut der Zentralbank Neuseelands, bei Issing (1992c), S. 6. 
Mitgliedern der ersten beiden Ländergruppen zusammensetzt, kann nicht automatisch von dem Wunsch nach einer strikten Antiinflationspolitik ausgegangen werden. Insbesondere die Länder der zweiten Gruppe realisierten ihre erfolgreiche Politik erst, als das EWS bestand und eine autonome Geldpolitik nicht mehr möglich war. Die vorher nicht geplante asymmetrische Funktionsweise des EWS mit der Dominanz der D-Mark, die sich zur Ankerwährung entwickelt hat, zwang die übrigen Zentralbanken, sich an den Kurs der Bundesbank anzukoppeln, wenn sie ihre Währungen nicht abwerten wollten. ${ }^{2}$ Die wirtschaftliche Konvergenz insbesondere seit den achtziger Jahren ist also keine Folge der EWS-Regeln, wie sie ursprünglich gedacht waren, sondern im Gegenteil das Ergebnis des bewußten Außerkraftsetzens dieser Regeln, indem die stabilitätspolitische Asymmetrie dominierte. Neben der disziplinierenden Wirkung des EWS begünstigten aber auch die Dollarschwäche sowie die fallenden Ölpreise die Rückkehr zur Stabilität. Von einer ,hausgemachten“ Stabilität kann also keine Rede sein.

Die EZB stellt zudem einen gravierenden ordnungspolitischen Wandel dar, insofern als die bisherige Wettbewerbslösung durch die Verhandlungslösung ersetzt und die Geldpolitik künftig von allen Partnern gleichberechtigt mitbestimmt wird, was auch als Folge der abnehmenden Akzeptanz der D-Mark als Leitwährung zu verstehen ist. Der Maastricht-Vertrag entzieht dem EWS demnach die wettbewerbliche Grundlage, ohne andere Stabilitätsanreize oder Sanktionen an ihre Stelle zu setzen. Trotz der gestiegenen Konvergenz bestehen aber auch weiterhin unterschiedliche Unvollkommenheiten der Güter- und Faktormärkte innerhalb der EU, weshalb für die Partner unterschiedliche Möglichkeiten zum kurzfristig ausbeutbaren Trade-off zwischen Arbeitslosigkeit und Inflation existieren. Die Folge dieser strukturellen Unterschiede sind verschiedene national optimale Inflationsraten. Eine Einigung auf eine gemeinsame Rate führt dann aber zu einer durchschnittlichen Rate, die höher ist als die des inflationsärmsten Landes.

Die EZB bedarf demnach günstiger Rahmenbedingungen, damit sie mit einer gewissen Anfangsreputation starten kann. Eine dieser Bedingungen stellt ihre Unabhängigkeit dar, die sie laut Maastricht-Vertrag auch erhalten soll. ${ }^{3}$ Die Unterzeichner dieses Vertrags waren der Ansicht, daß die Reputation der Bundesbank auf die EZB übergehen könnte, wenn man deren Gesetzesgrundlage

2 Hierzu ausführlicher Gemper (1989) und Collignon (1991).

3 Die Unabhängigkeit allein ist aber noch keine Garantie für die Preisniveaustabilität, sondern nur eine gute Basis, wie das Beispiel der deutschen Reichsbank belegt. Vgl. Issing (1992c), S. 3, FN 10. 
als Vorlage übernimmt, was im EZB-Statut klar zum Ausdruck kommt. Teilweise geht die EZB-Satzung sogar über die deutschen Regelungen hinaus. So hat ihr Status durch die Festschreibung im Maastricht-Vertrag quasi Verfassungsrang. Auch mit der Standortwahl Frankfurt sollte ein Signal gesetzt werden, daß die europäischen Partner an die stabilitätsorientierte Tradition der Bundesbank anknüpfen wollen.

Allerdings wurden auch einige deutsche „Fehler“ übernommen, indem auch die EZB die allgemeine Wirtschaftspolitik unter Beachtung ihrer Aufgabe zu unterstützen hat und die Wechselkurspolitik in den Kompetenzbereich der Politiker fällt. Insbesondere dieser letzte Punkt kann sich als folgenschweres Problem für die Reputation der EZB erweisen. Zwar soll die Wechselkurspolitik im Einklang mit der am Preisniveau orientierten Geldpolitik der EZB stehen, ob sie dies aber tut oder nicht, entscheiden nicht die Zentralbanker, sondern Politiker, die primär politische Interessen berücksichtigen. Der wechselkurspolitische Aspekt ist im Maastricht-Vertrag also nicht glaubwürdig geregelt, da auf die Entpolitisierung des Instrumentes der Wechselkursänderung verzichtet wurde. Zudem entscheiden alle Partner der EU über die Wechselkurspolitik der EZB, unabhängig davon, ob sie Mitglied sind oder nicht.

Aber selbst wenn sich die Wechselkurspolitik nicht als Problem für die EZB erweisen sollte, können die Regelungen bezüglich der personellen Unabhängigkeit durch das Vorhandensein erheblicher Interpretationsspielräume reputationsmindernd wirken. Auch auf europäischer Ebene entscheiden ausschließlich Politiker über die Auswahl der Zentralbanker, das der EZB zugestandene Anhörungsrecht hierzu hat sich schon in Deutschland als in der Praxis bedeutungslos erwiesen. Der reputationssteigernden fachlichen Eignung der Zentralbanker wird durch den Wortlaut des Gesetzestextes in keiner Weise Rechnung getragen, und es ist nicht auszuschließen, daß bei der Auswahl politische Aspekte wichtiger sind als Expertenwissen. Auch die viel zu kurze Amtszeit der nationalen Zentralbankpräsidenten sowie die „laxe“ Wortwahl zur Beschreibung der vorzeitigen Entlassungsmöglichkeit bieten erhebliche politische Dominanzmöglichkeiten.

Insgesamt läßt der Gesetzestext zu viele Interpretationsspielräume. Die Unabhängigkeit ist nicht eindeutig definiert, und die Zielvorgabe ist nicht quantifiziert, weshalb alle Partner auch weiterhin unterschiedliche Vorstellungen über diese haben können. ${ }^{4}$

$4 \quad$ Vgl. Gischer (1993), S. 58ff., Issing (1992c), S. lff. und Lusser (1990), S. 91. Selbst die enge deutsche Interpretation der Preisniveaustabilität wandelte sich im Laufe der Zeit. Vgl. hierzu Teichmann (1988), S. 108f. 
Die tatsächliche Unabhängigkeit der Zentralbank wird im wesentlichen von ihrer informellen Macht abhängen, die sie durch die Akzeptanz in der Bevölkerung erhält. In Deutschland deckt sich bei unzulänglicher Gesetzesgrundlage der Bundesbank das Interesse der Bevölkerung mit dem primären Ziel der Zentralbank, was die Basis ihrer hohen Reputation ist. Es muß aber bezweifelt werden, daß sich in Europa bereits die gleiche Stabilitätskultur entwickelt hat. Diese ist ohnehin nicht in ganz Europa einheitlich, sondern von Land zu Land verschieden, wie sich bei der Analyse der Inflationsentwicklungen gezeigt hat. Auch die gesunkenen Inflationsdifferenzen können nicht als Indiz für eine gebrochene Inflationsmentalität der Partner überzeugen, da die Entwicklung dorthin immer wieder mit der Forderung der Partner nach einer expansiven Geldpolitik begleitet war. Zudem müssen auch die teilweise noch erheblich nach oben abweichenden Inflationsraten insbesondere der "Südstaaten“ berücksichtigt werden.

Wieder wird deutlich, daß insbesondere die Frage nach den Partnern der EZB deren Reputation bestimmen wird. Auch wenn zunächst ein stabilitätspolitischer Kern beginnt, werden die politischen Zwänge auf die EZB erheblich gröBer werden als die auf die nationalen Zentralbanken, allein schon durch die zunehmende Interessenvielfalt.

Ein erhebliches Problem wird auch die Koordination mit den anderen Bereichen der Wirtschaftspolitik darstellen, da die Fiskal- wie die Tarifpolitik auch weiterhin auf nationaler Ebene entschieden werden. Es wird dann nicht mehr nur die Unterstützung der Stabilitätspolitik durch eine nationale Regierung und eine nationale Arbeitnehmer- und Arbeitgeberseite notwendig sein, sondern mehrere nationale Organe mit unterschiedlicher Mentalität (z.B. in bezug auf die Streikbereitschaft) müssen ihre Politik mit der Geldpolitik abstimmen. Hierin wird das eigentliche Problem für die EZB liegen.

Erschwerend für die Koordination kommt hinzu, daß die Partner asymmetrisch von Datenänderungen getroffen werden. Selbst wenn man die positive Einschätzung der Kommission der EU übernimmt, daß die länderspezifischen Schocks aufgrund des steigenden intraindustriellen Handels bedeutungslos werden $^{5}$, bleibt das Problem bestehen, da die wirtschaftliche Entwicklung in den verschiedenen Ländern nicht symmetrisch verläuft und selbst symmetrische Schocks unterschiedliche Reaktionen hervorrufen.

5 Integrationsfortschritte durch feste Wechselkurse werden aber nur erzielt, wenn Preisniveaustabilität herrscht und die Wechselkurse Gleichgewichtskurse darstellen, d.h. die festen Nominalkurse mit den festen realen Kursen einhergehen. Vgl. dazu ausführlicher Schäfer (1993). 
Bei Wegfall des Wechselkurses müssen entweder die anderen Preise (Güterpreise und Löhne) um so flexibler reagieren bzw. die Mobilität des Faktors Arbeit muß enorm gesteigert werden, oder es erfolgt eine suboptimale Anpassung über die Mengen. Wie aber bereits die deutsch-deutsche Währungsunion zeigt, führt das Vorhandensein einer Währung zum Wegfall der Geldillusion mit der Folge der Lohnangleichung unabhängig von Produktivitätsunterschieden in Richtung auf den höchsten Einkommensstandard. Die Arbeitsmobilität wird durch den Binnenmarkt zwar begünstigt, trotzdem ist eine radikale Steigerung aufgrund sprachlicher und kultureller Barrieren eher unwahrscheinlich. Wird die dann notwendige Mengenanpassung als zu kostspielig erachtet ${ }^{6}$, wird schnell der Ruf nach finanziellen Hilfen aufkommen.

Zwar verbietet der Maastricht-Vertrag die Haftung für Schulden der Partner, aber letztlich stellen die EU und insbesondere die EWU eine Solidargemeinschaft dar, mit deren Hilfe man rechnen wird. Ihre Bestätigung findet diese Aussage in der Betonung des Finanzausgleichs im Delors-Bericht sowie in der bisherigen Entwicklung. ${ }^{7}$ Teilweise wird aber übersehen, daß die realwirtschaftlichen Probleme, die in Verbindung mit der Währungsintegration stehen, nicht die Folge der Währungsunion sind und somit kein Anspruch auf Transferleistungen besteht. Solche wären ein Akt der Solidarität. Betrachtet man allein die notwendigen Transferleistungen nach Ostdeutschland, muß aber bezweifelt werden, daß die erforderliche Solidarität auf europäischer Ebene zu dem um ein vielfach höheren notwendigen Finanztransfer bereits vorhanden ist. Ist dies tatsächlich nicht der Fall, wird der politische Druck auf die EZB zu einer akkommodierenden Geldpolitik zunehmen.

Aber auch auf die Problematik einer solchen umverteilungspolitischen Maßnahme muß hingewiesen werden, da die notwendigen Anpassungslasten auf Dritte (die europäischen Steuerzahler) abgewälzt würden und die notwendigen Anpassungen nicht oder erst später vorgenommen würden. Letztlich würde so nicht die Weiterentwicklung finanziert, sondern anti-ökonomisches Verhalten subventioniert. Auch der vermutete Sanktionsmechanismus, daß innerhalb der Währungsunion die hoch verschuldeten Partner von den Märkten „bestraft“ werden, indem sie eine höhere Risikoprämie von den betroffenen Ländern verlangen und somit zur Anpassung zwingen, wird noch weniger funktionieren, als dies bereits innerhalb des EWS gelang. Innerhalb der Währungsunion wird

6 Dies haben Großbritannien und Italien im Zuge der EWS-Krise von 1992 so empfunden mit der Folge der Suspendierung der Teilnahme ihrer Währungen am Wechselkursmechanismus. Vgl. Schäfer (1993), S. 99.

7 Die weniger entwickelten südeuropäischen Länder haben bereits ein umfassendes interregionales Transfersystem gefordert. 
sich ein einheitlicher Zinssatz bilden, und somit werden negative Effekte in Form höherer Zinsen auf die stabilitätskonformen Länder die Folge sein.

Zwar wurde versucht, durch die Aufstellung von Konvergenzkriterien zu gewährleisten, daß nur stabilitätsbewußte Partner die Währungsunion bilden, wie die Analyse dieser Kriterien aber gezeigt hat, werden sie ihrem vorgegebenen Ziel nicht gerecht. Völlig in die andere Richtung könnten sie sogar wirken, wenn trotz genannter Richtwerte diese gar nicht beachtet werden, sondern aufgrund politischer Überlegungen Ausnahmen die Regel werden. Diese Nichtbeachtung der selbst auferlegten Kriterien, was der Maastricht-Vertrag von vornherein zuläßt, würde der Reputation der EZB einen erheblichen Schaden zufügen.

Eine negative Wirkung auf die Reputation dürften bereits die Bestimmungen zum Übergang zur Währungsunion haben. Insbesondere die fehlende Forderung nach der Durchsetzung der Unabhängigkeit der nationalen Zentralbanken bereits in der zweiten Stufe muß als Problem für die Reputation der EZB gesehen werden. Durch den bewußten Verzicht der Nutzung der Übergangsphase als Testperiode für die Endstufe liegt ein fundamentales Glaubwürdigkeitsdefizit vor. Zwar wird die EZB erst in der dritten Stufe gegründet, trotzdem kann sie als direkter Nachfolger des Europäischen Währungsinstituts (EWI) gesehen werden, welches mit Errichtung der EZB entsprechend Artikel 23 I des Protokolls über die Satzung des EWI liquidiert wird.

$\mathrm{Zu}$ den Mitgliedern beider Einrichtungen (EWI und EZB) gehören die nationalen Zentralbanken. Wie glaubwürdig erscheint dann die Unabhängigkeit der Zentralbanken, wenn sie bei Gründung des „Vorläufers“ der EZB noch nicht akzeptiert wird. Insbesondere die Unabhängigkeit wird aber auch von den Politikern als notwendige Bedingung für eine Zentralbank propagiert, die primär ihrem Stabilitätsauftrag nachkommen, sich also Reputation aufbauen soll. Erschwerend für die Reputation kommt hinzu, daß die für den Maastricht-Vertrag verantwortlichen Politiker die Unabhängigkeit nicht nur als notwendige, sondern auch schon als hinreichende Bedingung betrachten. Wie im dritten Kapitel dieser Arbeit aber gezeigt wurde, erfordert die Gültigkeit dieser Aussage ganz spezielle Annahmen.

Für wie wichtig die Reputation der EZB tatsächlich gehalten wird, wird sich besonders dann zeigen, wenn zum endgültigen Starttermin am 1. Januar 1999 kein oder nur eine sehr geringe Anzahl von Mitgliedern die Konvergenzkriterien erfüllt. Im Interesse der Reputation müßte der Beginn der Währungsunion dann verschoben werden. Sollte jedoch der politische Wunsch nach Gründung 
der EZB dominieren, wird es aus deutscher Sicht zu erheblichen Reputationseinbußen kommen. Ökonomisch gesehen hätte Deutschland dann mehr Kosten als Nutzen von der Währungsunion, da entweder die realisierte Inflationsrate steigen oder im günstigsten Fall zwar weiterhin eine Antiinflationspolitik verfolgt würde, dies aber bei unglaubwürdiger Geldpolitik mit der Folge einer Stabilisierungskrise und steigender Arbeitslosigkeit, wie dies im modifizierten Modell von BG gezeigt wurde. ${ }^{8}$ Diese Reputationseinbuße hätte der Maastricht-Vertrag durch die Berücksichtigung von Anreizen bzw. Sanktionen für die Zentralbanker, die zur Einhaltung der Preisniveaustabilität zwingen, vermeiden können. ${ }^{9}$ Ein erfolgreiches Beispiel ist hier Neuseeland, in dessen Zentralbank-Verfassung nicht nur explizite Anreize vorgesehen sind, sondern auch berücksichtigt wird, daß die Zentralbank die Inflationsrate nicht allein bestimmt, da externe Einflüsse wirken können. ${ }^{10}$

8 Diese volkswirtschaftlichen Kosten in Form stärker schwankender Inflationsraten und zunehmender Konjunkturschwankungen wurden bereits in einer Studie der EGKommission (1990) zum Ausdruck gebracht.

9 Vgl. auch die Stellungnahme führender Wirtschaftswissenschaftler vom 11.6.1992.

10 Vgl. Schlesinger (1991), Schäfer (1993), Hoffmeyer (1990), Gaddum (1990), Kloten (1988b) und (1990), Tietmeyer (1990), Scharrer (1988), (1989) und (1991), Hasse (1989a), (1990), (1993a und b), Gäckle (1992), Caesar (1988), (1989) und Willms (1990a). 


\section{Literaturverzeichnis:}

Acheson, K., Chant, J.F.: The Choice of Monetary Instruments and the Theory of Bureaucracy, in: Public Choice, Spring 1972, S. 13-33.

Acheson, K., Chant, J.F.: Mythology and Central Banking, in: Kyklos, 1973a, S. 362-379.

Acheson, K., Chant, J.F.: Bureaucratic Theory and the Choice of Central Bank Goals, in: Journal of Money, Credit and Banking, 1973b, S. 637655.

Alesina, A.: Macroeconomic Policy in a Two-Party System as a Repeated Game, in: Quarterly Journal of Economics, Vol. 102, 1987, S. 651-678.

Alesina, A.: Macroeconomics and Politics, in: Fischer, S. (Ed.): NBER Macroeconomics Annual 1988, Cambridge and London, S. 13-52.

Alesina, A.: Politics and Business Cycles in Industrial Democracies, in: Economic Policy, Vol. 8, April 1989, S. 57-87.

Alesina, A., Sachs, J.: Political Parties and the Business Cycle in the United States, 1948-1984, in: Journal of Money, Credit and Banking, Vol. 20, 1988, S. 63-82.

Alesina, A., Tabellini, G.: Rules and Discretion with Noncoordinated Monetary and Fiscal Policies, in: Economic Enquiry, Vol. 25, 1987, S. 619630.

Arrow, K.J.: The Economics of Agency, in: Pratt, J.W., Zeckhauser, R.J. (Ed.): Principals and Agents - The Structure of Business, Boston, 1985, S. 37-51.

Backus, D., Driffill, J.: Inflation and Reputation, in: American Economic Review, Vol. 75, 1985a, S. 530-538.

Backus, D., Driffill, J.: Rational Expectations and Policy Credibility Following a Change in Regime, in: Review of Economic Studies, Vol. 52, 1985b, S. 211-221.

Bailey, J.M.: The Welfare Cost of Inflationary Finance, in: Journal of Political Economy, April 1956, S. 93-110.

Barro, R.J.: Reputation in a Model of Monetary Policy with incomplete Information, in: Journal of Monetary Economics, 17, 1986, S. 3-20.

Barro, R.J., Gordon, D.B.: Rules, Discretion and Reputation in a Model of Monetary Policy, in: Journal of Monetary Economics, 12, 1983a, S. 101121.

Barro, R.J., Gordon, D.B.: A Positive Theory of Monetary Policy in a Natural Rate Model, in: Journal of Political Economy, 1983b, S. 589-610.

Berthold, N.: Europa nach Maastricht - sind die währungspolitischen Fragen gelöst? in: Wirtschaftsdienst, 1992/1, S. 23-28. 
Blackburn, K., Christensen, M.: Monetary Policy and Policy Credibility: Theories and Evidence, in: Journal of Economic Literature, Vol. 27, 1989, S. 1-45.

Bofinger, P.: Politische Ökonomie und makroökonomische Vorteile der Europäischen Zentralbank, in: Bofinger, P. u.a. (Hrsg.): Währungsunion oder Währungschaos? Wiesbaden, 1993, S. 43-57.

Brunetti, A.: Politisches System und Wirtschaftswachstum, Zürich u.a., 1992.

Caesar, R.: Der Handlungsspielraum von Notenbanken, Baden-Baden, 1981.

Caesar, R.: Notenbanken im Spannungsfeld der Politik, in: Wirtschaftsdienst, 1982/11, S. 569-576.

Caesar, R.: Bundesbank-Autonomie: Internationale Bedrohungen? in: Wirtschaftsdienst, 1988/3, S. 124-129.

Caesar, R.: Die „Autonomie“ der Deutschen Bundesbank - Ein Modell für Europa? in: Hasse, R., Schäfer, W. (Hrsg.): Europäische Zentralbank, Göttingen, 1990, S. 111-127.

Canzoneri, M.B.: Monetary Policy Games and the Role of Private Information, in: American Economic Review, 75, 1985, S. 1056-1070.

Clausen, V., Willms, M.: Unabhängigkeit der Zentralbank, in: Wirtschaftswissenschaftliches Studium, Heft 12, 1993, S. 605-610.

Collignon, S.: Asymmetrie und Reversibilität im EWS: Bedroht die deutsche Einheit die Ankerfunktion der DM? in: Westphal, A. u.a. (Hrsg.): Wirtschaftspolitische Konsequenzen der deutschen Vereinigung, Frankfurt am Main, New York, 1991, S. 115-141.

Collignon, S.: Krise im Europäischen Währungssystem oder: Wie gewinnt die Europäische Währungsunion ihr Vertrauen zurück? in: Bofinger, P. u.a. (Hrsg.): Währungsunion oder Währungschaos? Wiesbaden, 1993, S. 105121.

Cukierman, A.: Central Bank Behavior and Credibility: Some Recent Theoretical Developments, in: Federal Reserve Bank of St. Louis, Vol. 68, No. 5, May 1986, S. 5-16.

Cukierman, A.: Central Bank Strategy, Credibility, and Independence: Theory and Evidence, Cambridge u.a., 1992.

Cukierman, A., Meltzer, A.H.: A Theorie of Ambiguity, Credibility and Inflation under Discretion and Asymmetric Information, in: Econometrica, Vol. 54, No. 5, Sep. 1986a, S. 1099-1128.

Cukierman, A., Meltzer, A.H.: The Credibility of Monetary Announcements, in: Neumann, M.J.M. (Ed.): Monetary Policy and Uncertainy, BadenBaden, 1986b, S.39-67. 
Cukierman, A., Meltzer, A.H.: A Positive Theory of Discretionary Policy, the Cost of a Democratic Government, and the Benefits of a Constitution, in: Economic Inquiry, Vol. 24, 1986c, S. 367-388.

Delors, J. u.a.: Ausschuß zur Prüfung der Wirtschafts- und Währungsunion: Bericht zur Wirtschafts- und Währungsunion in der Europäischen Gemeinschaft, O.O., 1989, („Delors-Bericht").

Diffenbach, J., Higgins, R.B.: Strategic Credibility Can Make a Difference, in: Business Horizons, Heft 3, 1987, S. 13-18.

Dittus, P.: Die Wahl der Geldverfassung, Köln u.a., 1987.

Dornbusch, R.: Ireland's disinflation, in: Economic Policy, April 1989, S. 173-209.

Driffill, J.: Macroeconomic Policy Games with Incomplete Information, in: European Economic Review, 32, 1988, S. 533-541.

Duisenberg, W.F.: Stabilitätswirkungen des EWS, Brüssel, 15. Dezember, in: Deutsche Bundesbank: Auszüge aus Presseartikeln, Nr. 88, 29. Dezember 1986, S. 1f.

Eucken, W.: Grundsätze der Wirtschaftspolitik, Tübingen, 1990.

Eijffinger, S.C.W.: Konvergenz in der Geldpolitik: das Beispiel DeutschlandFrankreich, in: Weber, M. (Hrsg.): Europa auf dem Weg zur Währungsunion, Darmstadt, 1991, S. 171-192.

Fellner, W.: Towards a Reconstruction of Macroeconomics: Problems of Theory and Policy, in: American Enterprise Institute for Public Policy Research, Washington, 1976.

Fellner, W.: The Credibility Effects and Rational Expectations: Implications of the Gramlich Study, in: Brooking Papers on Economic Activity, No. 1, 1979, S. 167-189.

Filc, W.: Zentralbankautonomie und geldpolitische Effizienz, in: WSI Mitteilungen, 2/1994, S. 698-708.

Fischer, S.: Long-Term Contracts, Rational Expectations, and the Optimal Money Supply Rule, in: Journal of Political Economy, 85, 1, 1977, S. 191-205.

Francke, H.H.: $\mathrm{Zu}$ einigen ablauf- und ordnungspolitischen Problemen einer Europäischen Zentralbank aus polit-ökonomischer Sicht, in: Hasse, R., Schäfer, W. (Hrsg.): Europäische Zentralbank, Göttingen, 1990, S. 134151.

Franz, O.: Die Europäische Zentralbank, in: Franz, O. (Hrsg.): Die Europäische Zentralbank, Bonn, 1990, S. 13-28.

Frey, B.S.: Theorie demokratischer Wirtschaftspolitik, München, 1981. 
Frey, B.S., Schneider, F.: An Empirical Study of Politico-Economic Interaction in the United States, in: The Review of Economics and Statistics, Vol. 60, 1978, S. 174-183.

Frey, B.S., Schneider, F.: An Econometric Model with an Endogenous Government Sector, in: Public Choice, Vol. 34, 1979, S. 29-43.

Fricke, D.: Verteilungswirkungen der Inflation, Baden-Baden, 1981.

Friedman, J.: A Noncooperative Equilibrium for Supergames, in: Review of Economic Studies, Vol. 38, 1971, S. 1-12.

Friedman, M.: A Program for Monetary Stability, New York, 1959.

Funke, N.: Die Glaubwürdigkeit von Wirtschaftsreformen: Bedeutung, Ursachen und Ansatzpunkte zur Lösung von Glaubwürdigkeitsproblemen, in: Weltwirtschaft, 1991, Heft 2, S. 175-186.

Gaddun, J.W.: Chancen und Risiken der europäischen Währungsintegration, in: Franz, O. (Hrsg.): Die Europäische Zentralbank, Bonn, 1990, S. 141149

Gäckle, T.: Die Weiterentwicklung des Europäischen Währungssystems zur Europäischen Währungsunion, Baden-Baden, 1992.

Gemper, B.B.: Die Deutsche Bundesbank - Vorbild, aber kein Modell, in: Der Arbeitgeber, 41/1989, S. 17-19.

Genscher, H-D.: Memorandum für die Schaffung eines europäischen Währungsraumes und einer Europäischen Zentralbank, in: Deutsche Bundesbank: Auszüge aus Presseartikeln, Nr. 15, 1. März 1988, S. $6 f$.

Giavazzi, F., Giovannini, A.: Limiting Exchange Rate Flexibility, in: MIT Press, Cambridge, Massachusetts, 1989.

Giavazzi, F., Pagano, M.: The Advantage of Tying One's Hand, in: European Economic Review, 1988, 32, S. 1055-1082.

Gischer, H.: Stabilität des Geldwertes, Währungsordnung und Europäische Zentralbank, in: List-Forum, Vol. 19, Heft 1, 1993, S. 50-63.

Haberler, G.: Notes on Rational and Irrational Expectations, in: American Enterprise Institute, Reprint No. 111, March 1980.

Hagen, J. von: Monetary Policy Delegation and Fixed Exchange Rates: Credibility, Reputation, and Inflation in the EMS, in: Center for Global Business, Indiana, November 1989.

Hamacher, S.: Glaubwürdigkeitsprobleme in der Geldpolitik, Stuttgart u.a., 1995.

Hansmeyer, K.H.: Wandlungen im Handlungsspielraum der Notenbank? in: Andeae, C. A. u.a. (Hrsg.): Geldtheorie und Geldpolitik, Berlin, 1968, S. 155-166. 
Hasse, R.: Die Vorschläge zur Weiterentwicklung des EWS: Visionäres und Realisierbares, in: Wirtschaftswissenschaftliches Studium, Heft 8, 1986, S. 409-411.

Hasse, R.: Die Europäische Zentralbank: Perspektive für eine Weiterentwicklung des europäischen Währungssystems, Gütersloh, 1989a.

Hasse, R.: Die Europäische Integration, Bilanz und Perspektiven, in: Wirtschaftswissenschaftliches Studium, Heft 7, 1989b, S. 325-331.

Hasse, R.: Europäische Wirtschafts- und Währungsunion: Bilanz der Koordinierungsdefizite, in: Hasse, R., Schäfer, W. (Hrsg.): Europäische Zentralbank, Göttingen, 1990, S. 76-85.

Hasse, R.: Europäische Zentralbank, in: Das Parlament, Beilage: Aus Politik und Zeitgeschichte, B 7-8, 1992.

Hasse, R.: Die „Nach-Maastricht-Ära“: Neuorientierungen für den Übergang zur europäischen Währungsunion, in: Bofinger, P. u.a. (Hrsg.): Währungsunion oder Währungschaos? Wiesbaden, 1993a, S. 123-141.

Hasse, R.: Maastricht in der Krise, Ein Vorschlag zu mehr Glaubwürdigkeit, Discussion Papers in Economic Policy, No. 21, 1993b.

Hayek, F.A.: Entnationalisierung des Geldes, Tübingen, 1977.

Herr, H., Westphal, A.: Probleme der monetären Integration Europas, die Europäische Währungsunion und die deutsche Vereinigung, in: Westphal, A. u.a. (Hrsg.): Wirtschaftspolitische Konsequenzen der deutschen Vereinigung, Frankfurt/Main, New York, 1991, S. 75-114.

Heubes, J.: Inflationstheorie, München, 1989.

Hibbs, D.A.Jr.: Political Parties and Macroeconomic Policy, in: The American Political Science Review, Vol. 71, 1977, S. 1467-1487.

Hirschman, A.O.: Exit, Voice, and Loyalty, Cambridge, Massachusetts, 1970.

Hoffmeyer, E.: Europäische Währung und Europäische Zentralbank aus dänischer Sicht, in: Franz, O. (Hrsg.): Die Europäische Zentralbank, Bonn, 1990, S. 72-76.

Huß, H.J., Trapp, P.: Vorstellungen über eine alternative Geldordnung, in: Ehrlicher, W., Simmert, D.B. (Hrsg.): Geld- und Währungspolitik in der Bundesrepublik Deutschland, Beihefte zu Kredit und Kapital, Heft 7, 1982, S. 79-94.

IMF: International Monetary Funds: International Financial Statistics: Supplement on Price Statistics, Supplement Series, No. 12, December 1986, S. $4 f$.

IMF: International Monetary Funds: International Financial Statistics Yearbook, Vol. 48, 1995, S. $120 \mathrm{f}$.

Issing, O.: Die Unabhängigkeit der Bundesbank, Theoretisch umstritten praktisch bewährt, in: Ehrlicher, W., Simmert, D.B. (Hrsg.): Geld- und 
Währungspolitik in der Bundesrepublik Deutschland, Beihefte zu Kredit und Kapital, Heft 7, 1982, S. 49-60.

Issing, O.: Einführung in die Geldtheorie, 7. Auflage, München, 1990.

Issing, O.: Einführung in die Geldpolitik, München, 1992a.

Issing, O.: Geldpolitik im Spannungsfeld von Politik und Wissenschaft, in: Albeck, H. (Hrsg.): Wirtschaftsordnung und Geldverfassung, Göttingen, 1992b, S. 46-56.

Issing, O.: Die Unabhängigkeit der Notenbank und Geldwertstabilität, in: Deutsche Bundesbank: Auszüge aus Presseartikeln, Nr. 79, 10.November 1992 c, S. 1-8.

Jarchow, H.J.: Diskretionäre Geldpolitik, Zeitinkonsistenz und Politikglaubwürdigkeit, in: Wirtschaftswissenschaftliches Studium, 2/1993, S. 145151.

Kane, E.J.: Politics and FED Policy-making, in: Journal of Monetary Economics, 6/1980, S. 199-211.

Kastner, S.: Glaubwürdigkeit und Reputation der Geldpolitik, Wiesbaden, 1994.

Kirsch, G.: Politische Grenzen der Geldpolitik, in: Ehrlicher, W., Simmert, D.B. (Hrsg.): Geld- und Währungspolitik in der Bundesrepublik Deutschland, Beihefte zu Kredit und Kapital, Heft 7, 1982, S. 33-47.

Kloten, N.: Zur Anatomie geldpolitischer Entscheidungen des Zentralbankrates, in: Dürr, E., Sieber, H. (Hrsg.): Weltwirtschaft im Wandel, Bern, Stuttgart, 1988a, S. 401-416.

Kloten, N.: Wege zu einem Europäischen Zentralbanksystem, in: EuropaArchiv, Folge 11, 1988b, S. 285-298.

Kloten, N.: Auf dem Weg zur Europäischen Währungsunion: Der „DelorsBericht", in: Franz, O. (Hrsg.): Die Europäische Zentralbank, Bonn, 1990, S. 241-250.

Kösters, W.: Die „Autonomie“ der Deutschen Bundesbank - Ein Modell für Europa? Korreferat zu Caesar, R., in: Hasse, R., Schäfer, W. (Hrsg.): Europäische Zentralbank, Göttingen, 1990, S. 128-133.

Kösters, W.: Europäische Zentralbank und Preisniveaustabilität, in: Hamburger Jahrbuch für Wirtschafts- und Gesellschaftspolitik, 36, 1991, S. 155167.

Kommission der EG: „Ein Markt - Eine Währung“, in: Europäische Wirtschaft, Nr. 44, 1990.

Kremers, J.M.: Gaining Policy Credibility for a Disinflation, in: IMF Staff Papers, Vol. 39, No. 1, 1990, S. 116-145.

Kreps, D.M., Wilson, R.: Reputation and Imperfect Information, in: Journal of Economic Theory, Vol. 27, 1982a, S. 253-279. 
Kreps, D.M., Wilson, R.: Sequential Equilibria, in: Econometrica, Vol. 50, 1982b, S. 863-894.

Kydland, F.E., Prescott, E.C.: Rules Rather than Discretion: The Inkonsistency of Optimal Plans, in: Journal of Political Economy, 1977/1, S. 473490.

Leigh-Pemberton, R.: Europäische Währung und Europäische Zentralbank aus britischer Sicht, in: Franz, O. (Hrsg.): Die Europäische Zentralbank, Bonn, 1990, S. 42-50.

Loef, H.E.: Diskretionäre Geldpolitik, rationale Erwartungen und Politikglaubwürdigkeit, in: Jahrbuch für Sozialwissenschaft, 39, 1988, S. 361-375.

Loef, H.E.: Der Delors-Plan und die Anforderungen an eine gemeinsame europäische Geldpolitik, Korreferat zu Willms, M., in: Hasse, R., Schäfer, W. (Hrsg.): Europäische Zentralbank, Göttingen, 1990, S. 106-110.

Lucas, R.Jr.: Expectations and the Neutrality of Money, in: Journal of Economic Theory, Vol. 4, 1972, S. 103-124.

Lucas, R.Jr.: Some International Evidence on Output-Inflation Tradeoffs, in: American Economic Review, Vol. 63, 1973, S. 326-334.

Lusser, M.: Europäische Währung und Europäische Zentralbank aus schweizerischer Sicht, in: Franz, O. (Hrsg.): Die Europäische Zentralbank, Bonn, 1990, S. 90-95.

Marlow, M.L.: Central bank credibility and forecasting, in: Applied Economics, 1991, 23, S. 471-478.

Masson, P.R., Symansky, S.A.: Evaluating Policy Rules under Imperfect Credibility, in: IMF Working Paper, December 1991.

McCallum, B.T.: The Case for Rules in the Conduct of Monetary Policy, in: Weltwirtschaftliches Archiv, Band 123, 1987, S. 415-428.

Melitz, J.: Monetary Discipline and Cooperation in the European Monetary System: a Synthesis, in: Giavazzi, F. u.a. (Ed.): The European Monetary System, Cambridge, 1988.

Milgrom, P., Roberts, J.: Predation, Reputation and Entry Deterrence, in: Journal of Economic Theory, Vol. 27, 1982a, S. 280-312.

Milgrom, P., Roberts, J.: Limit Pricing and Entry under Incomplete Information: An Equilibrium Analysis, in: Econometrica, Vol. 50, 1982b, S. 443-459.

Mino, K., Tsutsui, S.: Reputational Constraint And Signalling Effects In A Monetary Policy Game, in: Oxford Economic Papers, 42, 1990, S. 603619.

Neumann, M.J.M.: Deutsche Geldpolitik: Verfahren und Probleme, in: Finanzmarkt- und Portfolio Management, 1988, Heft 4, S. 47-55. 
Neumann, M.J.M.: Central Bank Independence as a Prerequisite of Price Stability, in: European Community (Ed.): The Economics of European Monetary Union, Vol. 2: Contributions of Independent Economists, Brüssel, 1990.

Neumann, M.J.M.: Bindung durch Zentralbankunabhängigkeit, in: Albeck, H. (Hrsg.): Wirtschaftsordnung und Geldverfassung, Göttingen, 1992, S. 6273.

Niskanen, W. A.: Bureaucrats and Politicians, in: Journal of Law and Economics, Vol. 18, 1975, S. 617-643.

Nordhaus, W.: The Political Business Cycle, in: Review of Economic Studies, Vol. 42, 1975, S. 169-190.

al-Nowaihi, A., Levine, P.: Can Reputation Resolve The Monetary Policy Credibility Problem? in: Discussion Papers In European Economic Studies, October 1993.

OECD: Main Economic Indicators, February 1996, S. 18.

Olson, M.: Die Logik des kollektiven Handelns, Tübingen, 1968.

Pearlman, J.: Can Reputation Decrease Inflationary Expectations? in: Centre for Economic Forecasting, Discussion Paper, No. 07-89, April 1989.

Persson, T.: Credibility of Macroeconomic Policy, in: European Economic Review, 32, 1988, S. 519-532.

Persson, T., Tabellini, G.: Macroeconomic Policy, Credibility and Politics, Chur u. a., 1991.

Pfister, J.: Grundzüge einer „Soziotheorie“ der Inflation, Berlin, 1981.

Pohl, R.: Theorie der Inflation, München, 1981.

Puchta, D.G.: Elemente einer politisch-ökonomischen Theorie der schleichenden Inflation mit endogenisiertem Zentralbankverhalten, Freiburg, 1981.

Rogoff, K.: The Optimal Degree of Commitment To An Intermediate Monetary Target, in: Quarterly Journal of Economics, 1985, S. 1169-1189.

Rogoff, K.: Reputational Constraints on Monetary Policy, in: CarnegieRochester Conference Series on Public Policy, 26, 1987a, S. 141-182.

Rogoff, K.: Equilibrium Political Budget Cycles, in: NBER Working Paper, No. 2428, Washington, $1987 \mathrm{~b}$.

Rogoff, K., Siebert, A.: Elections and Macroeconomic Policy Cycles, in: Review of Economic Studies, Vol. 55, 1988, S. 1-16.

Roubini, N., Sachs, J.: Political and Economic Determinants of Budget Deficits in the Industrial Democracies, in: NBER Working Paper, No. 2628, Washington, 1988.

Roubini, N., Sachs, J.: Government Spending and Budget Deficits in the Industrial Countries, in: Economic Policy, No. 8, April 1989, S. 100-132. 
Sachs, J., Wyplosz, C.: France under Mitterand, in: Economic Policy, Vol. 2, April 1986, S. 261-313.

Sargent, T.J., Wallace, N.: Some Unpleasant Monetarist Arithmetic, in: Federal Reserve Bank of Minneapolis, Quarterly Review, Vol. 5, 1981, No. 3.

Schäfer, W.: EWS oder flexible Wechselkurse? in: Bofinger, P. u.a. (Hrsg.): Währungsunion oder Währungschaos? Wiesbaden, 1993, S. 95-103.

Scharrer, H.E.: Eine Zentralbank für Europa? in: Integration, 11.Jg., 3/1988, Beilage zur Europäischen Zeitung, 7/1988, S. 95-102.

Scharrer, H.E.: Stabilitätspolitischer Grundkonsens erforderlich, in: Wirtschaftsdienst, 1989/6, S. 279-283.

Scharrer, H.E.: EWWU: Klammer oder Sprengsatz? in: Wirtschaftsdienst, 1991/12, S. 594f.

Schlesinger, H.: Der Weg zu einer Europäischen Wirtschafts- und Währungsunion, in: Wirtschaftsdienst, 1991/5, S. 232-238.

Schlüter, P.W.: Die Europäische Wirtschafts- und Währungsunion: Anmerkungen zur Regierungskonferenz, in: Integration, 14.Jg., 3/1991, Beilage zur Europäischen Zeitung 7-8/1991, S. 106-114.

Schneider, F., Frey, B.S.: Politico-Economic Models of Macroeconomic Policy - A Review of the Empirical Evidence, in: Willett, T.D.(Ed.): Political Business Cycles - The Political Economy of Money, Inflation and Unemployment, Durham and London, 1988, S. 239-275.

Simon, H.C.: Rules versus Authority in Monetary Policy - A Concrete Example, in: Journal of Political Economy, Vol. 44, 1936, S. 1-30.

Socher, K.: Das Vorhaben einer Europäischen Währungsunion aus österreichischer Sicht, in: Weber, M. (Hrsg:): Europa auf dem Weg zur Währungsunion, Darmstadt, 1991, S. 369-391.

Stellungnahme führender Wirtschaftswissenschaftler: Die währungspolitischen Beschlüsse von Maastricht: Eine Gefahr für Europa, in: Deutsche Bundesbank: Auszüge aus Presseartikeln, Nr. 41, Juni 1992, S. 13.

Studt, D.: Rechtsfragen einer europäischen Zentralbank, Berlin, 1993.

Szass, A.: Die Währungspolitik in der EG - aus Sicht der Niederländischen Zentralbank, in: Deutsche Bundesbank: Auszüge aus Presseartikeln, Nr. 45, 7. Juni 1989, S. 5-8.

Tatom, J.A.: The Welfare Cost of Inflation, in: Federal Reserve Bank of St. Louis Review, November 1976, S. 9-22.

Teichmann, U.: Grundriß der Konjunkturpolitik, München, 1988.

Theurl, T.: Eine gemeinsame Währung für Europa, Innsbruck, 1992.

Tietmeyer, H.: Grundsätze für ein europäisches Zentralbanksystem, in: Franz, O. (Hrsg.): Die Europäische Zentralbank, Bonn, 1990, S. 150-163. 
Tietmeyer, H.: Währungsunion - ein Weg ohne Umkehr, in: Integration, 15. Jg., 1/1992, Beilage zur Europäischen Zeitung, 1/1992, S. 17-24.

Tietmeyer, H.: Europäische Währungsunion und Notenbank als Gestaltungsaufgabe, in: Bofinger, P. u.a. (Hrsg.): Währungsunion oder Währungschaos? Wiesbaden, 1993, S. 25-42.

Tower, E.: More on the Welfare Cost of Inflationary Finance, in: Journal of Money, Credit and Banking, November 1971, S. 850-860.

Vaubel, R.: Überholte Glaubenssätze, in: Wirtschaftsdienst, 1989/6, S. 276279.

Vaubel, R.: Currency Competition and European Monetary Integration, in: Economic Journal, Vol. 100, 1990, S. 936-946.

Vaubel, R.: Die Politische Ökonomie einer Europäischen Zentralbank, Probleme und Lösungsvorschläge, in: Bofinger, P. u.a. (Hrsg.): Währungsunion oder Währungschaos? Wiesbaden, 1993, S. 59-64.

Vickers, J.: Signalling in a Model of Monetary Policy with incomplete Information, in: Oxford Economic Papers 38, 1986, S. 443-455.

Wagner, H.: Stabilitätspolitik - Theoretische Grundlagen und institutionelle Alternativen, München und Wien, 1992.

Weber, A.: Reputation and Credibility in the European Monetary System, in: Economic Policy, April 1991, S. 57-101.

Willeke, C.: Zentralbanken und Inflation, Berlin, 1993.

Willms, M.: Der Delors-Plan und die Ausgestaltung einer gemeinsamen europäischen Geldpolitik, in: Hasse, R., Schäfer, W. (Hrsg.): Europäische Zentralbank, Göttingen, 1990a, S. 86-105.

Willms, M.: Grundprobleme eines Europäischen Zentralbankstatuts, in: Wirtschaftsdienst, 1990b/11, S. 551-556.

Woll, A.: Die Unabhängigkeit der Deutschen Bundesbank muß gesichert werden! in: Wirtschaftsdienst, 1988/3, S. 122-124.

Woll, A.: Die Unabhängigkeit der Europäischen Zentralbank: Dogma oder Notwendigkeit, in: Weber, M. (Hrsg.): Europa auf dem Weg zur Währungsunion, Darmstadt, 1991, S. 157-170.

Zimmermann, H., Henke, K.-D.: Einführung in die Finanzwissenschaft, München, 1987. 


\title{
SCHRIFTEN ZUR WIRTSCHAFTSTHEORIE UND WIRTSCHAFTSPOLITIK
}

\section{Herausgegeben von Rolf Hasse, Wolf Schäfer,}

\author{
Thomas Straubhaar, Klaus W. Zimmermann
}

Band 1 Lars Bünning: Die Konvergenzkriterien des Maastricht-Vertrages unter besonderer Berücksichtigung ihrer Konsistenz. 1997.

Band 2 Andreas Henning: Beveridge-Kurve, Lohnsetzung und Langzeitarbeitslosigkeit. Eine theoretische Untersuchung unter Berücksichtigung des Insider-Outsider-Ansatzes und der Entwertung des Humankapitals. 1997.

Band 3 Iris Henning: Die Reputation einer Zentralbank. Eine theoretische Untersuchung unter besonderer Berücksichtigung der Europäischen Zentralbank. 1997. 
Wolfgang Hermann Müller

\section{Prinzipien wirtschaftlichen Handelns und ihre Anwendung}

\section{Umriß einer Wirtschaftsphilosophie}

Frankfurt/M., Berlin, Bern, New York, Paris, Wien, 1996. 145 S., 1 Abb. ISBN 3-631-30477-3 · br. DM 48.-*

Die enge Verbindung von Wirtschaft und Weltgeschehen verlangt nach einer exakten Untersuchung ihrer Notwendigkeit, Möglichkeiten und Grenzen. Die Ökonomie wird auf ihre erlernbaren theoretischen Prinzipien, die Möglichkeiten ihrer Anwendung und die Bedingungen ihrer praktischen Durchsetzbarkeit hin untersucht. Ergebnis ist eine gründliche Neubestimmung des Verhältnisses von Theorie, Anwendung und Praxis. Die historisch bekannten Wirtschaftsschulen und -richtungen werden auf ihren Geltungsanspruch hinterfragt. Politische und ideologische Rahmenbedingungen werden in ihrem Einfluß auf Privat- und Gemeinwirtschaft erläutert und Modelle zur Überprüfung von Wirtschaftspolitik und Wirtschaftsethik aufgezeigt.

Aus dem Inhalt: Wissenschaftsanspruch, Definition und Methoden der Ökonomie - Einfluß von politischen und ideologischen Rahmenbedingungen . Privat- und Gemeinwirtschaft - Wirtschaftsschulen und Wirtschaftsmodelle . Wirtschaftsrecht und Wirtschaftsethik - Ökonomische Begriffsbildung und Wissenschaft · Ökonomie und Philosophie

Frankfurt/M · Berlin · Bern · New York · Paris · Wien

Auslieferung: Verlag Peter Lang AG

Jupiterstr. 15, CH-3000 Bern 15

Telefon (004131) 9402131

*inklusive Mehrwertsteuer

Preisänderungen vorbehalten 